\title{
METHODS TO REDUCE NON-LINEAR MECHANICAL SYSTEMS FOR INSTABILITY COMPUTATION
}

\author{
J-J. Sinou, F. Thouverez and L. Jézéquel \\ Laboratoire de Tribologie et Dynamique des Systèmes UMR-CNRS 5513 \\ Ecole Centrale de Lyon, 36 avenue Guy de Collongue \\ 69134 Ecully Cedex, France \\ jean-jacques.sinou@ec-lyon.fr
}

\begin{abstract}
Non-linear dynamical structures depending on control parameters are encountered in many areas of science and engineering. In the study of non-linear dynamical systems depending on a given control parameter, the stability analysis and the associated non-linear behaviour in a near-critical steady-state equilibrium point are two of the most important points; they make it possible to validate and characterize the non-linear structures. Stability is investigated by determining eigenvalues of the linearized perturbation equations about each steady-state operating point, or by calculating the Jacobian of the system at the equilibrium points. While the conditions and the values of the parameters which cause instability can be investigated by using linearized equations of motion, studies of the nonlinear behaviour of vibration problems, on the other hand, require the complete non-linear expressions of systems. Due to the complexity of non-linear systems and to save time, simplifications and reductions in the mathematical complexity of the non-linear equations are usually required. The principal idea for these non-linear methods is to reduce the order of the system and eliminate as many non-linearities as possible in the system of equations.

In this paper, a study devoted to evaluating the instability phenomena in non-linear models is presented. It outlines stability analysis and gives a non-linear strategy by constructing a reduced order model and simplifying the non-linearities, based on three non-linear methods: the centre manifold concept, the rational approximants and the Alternating Frequency/Time domain method. The computational procedures to determine the reduced and simplified system via the centre manifold approach and the fractional approximants, as well as the approximation of the responses as a Fourier series via the harmonic balance method, are presented and discussed. These nonlinear methods for calculating the dynamical behaviour of non-linear systems with several degrees-of-freedom and non-linearities are tested in the case of mechanical systems with many degrees-of-freedom possessing polynomial non-linearities. Results obtained are compared with those estimated by a classical Runge-Kutta integration procedure.

Moreover, an extension of the centre manifold approach using rational approximants is proposed and used to explore the dynamics of non-linear systems, by extending the domain of convergence of the non-linear reduced system and evaluating its performance and suitability.
\end{abstract}

\section{Introduction}

In the field of engineering, the need for consideration of non-linear effects in the description of a dynamical system is well recognized. Due to the fact that many structural systems of practical interest possess complex non-linear behaviour, there has been a crucial and strong development in the treatment of non-linear differential equations and in the application of non-linear methods to enable the analysis of vibration problems. These non-linear treatments are commonly applied in a wide range of mechanical engineering problems where it is impossible to ensure the stability of a non-linear system and self-excited vibrations may be generated ([191], [156], [192], [194] and [193]).Though the instability of the equilibrium position of non-linear systems and the associated stability condition can be investigated by considering a linear stability theory, the complete expressions of non-linear systems need to be taken into account in order to obtain the non-linear behaviour of systems. As is well known, a common procedure for estimating the non-linear dynamical behaviour of systems is the numerical integration procedure. However, the use of this approach for non-linear models with many degrees of freedom can be rather expensive and requires considerable resources both in terms of computation time and data storage. In these cases, non-linear methods for reducing, simplifying and approximating the non-linear responses can be applied. 
The main objective of these non-linear methods is to extract and characterize the non-linear behaviours of structural systems by using non-linear interpretations of modes and non-linear extensions of principal coordinate transformations ([188], [189], [153], [154], [134], [135], [164]). These various non-linear methods have been investigated for obtaining a low-dimensional and simplified system near the Hopf bifurcation point without losing the contribution of the non-linear terms in the field of non-linear mechanical autonomous systems depending on one parameter. One of the most powerful and frequently used reduction methods then is the centre manifold approach based on the reduction of the dimension of the original system ([135], [70], [99], [82], [167], [170] and [172]); the lower-dimensional non-linear system has the same non-linear vibrational behaviour as the original one. One of the bases for simplifying non-linear models is the method of normal forms that allows us to eliminate as many non-linear terms as possible through a non-linear change of variables ([2], [125], [133], [7], [34], [22], [23], [92], [54], [93], [83], [126],[171], [72], [203], [6], [204], and [206]). Even if the method of normal forms is usually applied after the centre manifold approach, other non-linear simplification methods such as the Pade approximants should be applied ([19], [21], [3] and [18]); all these non-linear methods possess advantages and disadvantages; the fractional rational approximants present a great advantages for obtaining information about the non-linear function outside its circle of convergence, and for more rapidly evaluating the function within its circle of convergence. However, the use of these fractional approximants are not commonly used after the centre manifold reduction.

In this study we propose to apply non-linear methods to reduce and simplify non-linear systems near the Hopf bifurcation point. Applications of these ideas will be investigated for mechanical dynamical systems with polynomial non-linearities. After the estimate of the stable and unstable areas due to the variation of given parameters, three non-linear methods will be successively applied to investigate the non-linear behaviour of dynamical systems around the Hopf bifurcation point. The centre manifold approach will be used first, to reduce the original non-linear systems. Secondly, the rational fractional approximants will be applied to simplify the non-linear reduced system. Finally, a harmonic balance method, that enables us to obtain the solution as an assumed time function, will be developed. The choices of each non-linear method and more particularly the use of the rational approximants to simplify non-linear systems instead of the normal form approach will be justified and argued.

This paper is divided into four sections. The purpose of Section 1 is to present a survey of the non-linear methods ap-plied in mechanical engineering to reduce, simplify and to approximate the non-linear behaviour of structural systems. These non-linear methods reduce the number of equations of the original system and simplify the non-linear terms in order to obtain a simplified system without losing the dynamics of the original system as well as the essential contributions of non-linear terms. More particularly, the basic concept of the centre manifold approach, the normal form approach and the rational fractional approximants procedure are developed; the theory, the classical procedures to obtain the reduced and simplified systems by using each non-linear method, and the advantages and disadvantages of these non-linear methods are discussed.

Following the general theory, Section 2 presents an application of these non-linear methods for a two-degree-offreedom mechanical system with quadratic and cubic non-linearities. Attention is first focused on the stability theory and analysis. Second, the complete non-linear analysis of this mechanical system is developed: the centre manifold approach, the rational approximants and the Alternate Frequency/Time domain method are then considered in some detail. Each method is accompanied by a computational procedure with practical advice on usage: computational schemes with generalisation for $n$-dimensional non-linear system are given. Moreover, a presentation is given of comparative results obtained by these various non-linear methods and verifications by computational simulation with a classical numerical integration procedure; indications of the advantages and shortcoming of each method is discussed. Towards the end of this section, a new procedure using an extension of the centre manifold approach via the rational fractional approximants is presented: this methodology extends the domain of validity of non-linear systems reduced by using the centre manifold approach and applies the rational fractional approximants in order to enhance the convergence of the series expansions of the centre manifold theory. We will show that the interest of these rational approximants is that they require fewer terms than the associated Taylor series in order to obtain an accurate approximation of the behaviour of the complete non-linear system.

Finally, Section 3 is devoted to the application of this new non-linear strategy based on the centre manifold, the rational approximants and the Alternating Frequency/Time domain method, in order to study the non-linear dynamical behaviour of complex systems in near-critical steady-state equilibrium point. The goal of this section is to show the suitability, capability and computational advantages of the centre manifold extension via the rational approximants for a non-linear system with large degree-of-freedom: friction induced vibrations in a complex mechanical aircraft 
brake system that consists of a set of 15 coupled ordinary differential equations of the second order with polynomial non-linear terms are considered. Some basic concepts of aircraft brake systems, a concise survey of the associated friction instability mechanisms, and the non-linear mechanical model are first presented. Second, the results from stability analyses and determination of the non-linear behaviour of the complex system by applying the centre manifold extension via the fractional rational approximants are examined.

\section{Non-linear methods for dimension reduction and simplification}

\subsection{Overview of non-linear methods}

Even though the develop of non-linear procedures have attracted increasing attention in recent years, the earliest studies of non-linear vibrations were made by Poincare 120 years ago ([146] and [144]). It is only during the last 50 years that advances have been made by researchers in the problem of non-linear vibrations with one or many degrees of freedom in order to understand the non-linear behaviour of systems ( [7], [8], [112], [14], [101], [183], [1], [13], [39], [69], [188], [189], [153], [154], [134] and [135]).

A number of techniques have been used in the past by various researchers using numerical integration over time. These methods appear to be the most common solution for predicting the non-linear behaviour of systems. How-ever, they can be both extremely time-consuming and costly to perform in terms of storage requirements for large degreeof-freedom systems.

Various methods have been introduced by a number of researchers for calculating the non-linear responses of these complex systems. Conventional methods are approximation techniques that can be divided into two categories. The first category deals with the methods called the "small parameters techniques" such as the asymptotic method, the averaging method, the slowly varying coefficient method and a number of various perturbation techniques ([132], [1], [14], [56], [159], [101], [115], [139], [134] and [135]). One of the most important disadvantages of these methods that differ only slightly from one other is that all the non-linear terms in the equations of motion are assumed to be small and only proportional to a given parameter. The second category of approximation techniques deals with the Galerkin method, the Ritz method, the harmonic balance methods, trigonometric collocation, etc. They require an initial assumption about the form of the solution of the non-linear system as a function of time and the estimated approxi-mate response is obtained by minimizing the residuals of the equations of motions ([196], [197], [184], [118], [81], [120], [152] [117], [104], [134], [135], [78], [202], [131], [130], [158] [30] and [91]). For this last category, the most popular methods for approximating the non-linear responses of systems are the harmonic balance methods where the non-linear solution is assumed to be a truncated Fourier series (harmonic balance method (HBM) [138], [12] and [157], incremental harmonic balance method (IHBM) [149], [111], [107], [108], [109], [143], [142], [113] and [95], alternate frequency/time domain method (AFT) [25], [129], [173] and [174], multi-harmonic balance method (MHB) [27], [26] and [98]). All these numerical methods are well-known and have been commonly used to solved non-linear problems in the fields of mechanical engineering.

Another standard approach is the linearized methods ([177], [178], [86], [85], [87], [89], [88] [29]). The principle of these methods is based on the well-known technique of equivalent linearization of Kryloff and Bogoliubov [101] and was extended by Iwan to multi-degree-of-freedom systems ([86], [85], [87], [89] and [88]). The purpose of this method is to replace the non-linear system by an equivalent linear system in which the difference between the two systems is minimized. Then, the solution of the associated linear system is taken as an approximation of the original non-linear problem. One of the advantages of this equivalent linearization procedure is that the resulting linear problem may be solved by any convenient technique, and this approach is easily mechanized and implemented.

An alternative approach to obtain an approximated solution of systems is to consider reduction and simplification techniques. The aims of these procedures is to obtain a simplified and reduced system that has approximately the same dynamical behaviour as the original one. Most of these schemes are concerned with linear reduction and simplification: in structural dynamics, modal analysis techniques are based on modal expansions ([94], [57], [65], [77], [37], [45], [71], [119], [67] and [66]). The purpose of this technique is to define the coordinate linear transformations from physical coordinates where the dynamical behaviour of structures are complex to modal coordinates where the structural system may be easily solved. Most mechanical systems are indeed quite complex due to their number of degree-of-freedom that leads to many vibrational modes; however, it may be demonstrated that certain modes often dominate in the frequency range of given operating points for structural systems. Then, by applying modal reduction 
methods, the low frequency modes or the most important modes may be used to define the reduced and simplified model.

Since non-linearities are often presented in structural systems, non-linear model reduction and simplification methods have been developed. During the last 30 years, Rosenberg ([153] and [154]) has defined the notion of the non-linear normal modes as synchronous motions with fixed relations between generalized coordinates, similar to the linear normal modes of classical vibration theory. This concept of non-linear normal modes has great potential for reduction techniques and has been studied by many researchers in recent years for weak and strong non-linear systems ([14], [96], [162], [161], [187], [188], [189], [186], [46], [5], [15], [163], [165], [164], [61], [160]). Shaw and Pierre [164] proposed a new definition of the non-linear normal modes in terms of invariant manifold approach and presented a sort of non-linear modal analysis to the non-linear problems: the non-linear normal mode takes place in an invariant manifold that is tangent to the linear modal subspaces at the point of equilibrium. A non-linear trans-formation is applied to relate the physical coordinates and the non-linear modal coordinates. The invariant manifold methodology has one aspect that seems to be very promising when searching for reduced models, since one non-linear normal mode is constructed by projecting the other modes over it by means of a non-linear relationship. These pro-jections contain the non-linear effects, and the performance of the reduced model is adapted to weak non-linearities by virtue of the power series used to obtain the approximation. However, the applicability of such non-linear modal analysis is restricted to a very small class of dynamical systems ([141] and [198]).

One of the most useful non-linear methods to reduce systems at near-equilibrium point is the centre manifold approach ([135], [70], [99], [82], [84], [62], [44], [28] [116], [167], [170] and [172]). This approach assumes that the non-linear dynamical system at near-equilibrium point is governed by the dynamics on the centre manifold when certain eigenvalues have zero real parts (and all other eigenvalues have negative real parts). It may be noted that the centre manifold appears to be an extremely powerful method due to the fact that if $m$ eigenval-ues of the $n$ eeignevalues of the non-linear system have zero real parts, then the number of equations for the non-linear system is reduced by $n-m$ by applying the centre manifold approach. However, this method can only be used for model reduction when the system has an eigenvalue with zero real parts at an equilibrium point. Usually, the centre manifold has complicated non-linear terms. In this case, the non-linear system can be simplified by using further non-linear coordinate transformations ([145], [146], [103], [2], [125], [7], [22], [23], [54], [93], [83]). The normal form theory is often applied after the centre manifold approach. The main objective of the method of normal forms is to obtain the simplest possible non-linear system by the use of successive non-linear co-ordinate transformations ([133], [92] and [70]). At the end of these non-linear transformations, only the resonant terms are retained: they cannot be eliminated and are essential to the non-linear system dynamics.

Moreover, there exist other non-linear methods called the rational approximants that simplify the non-linear terms by fractional series ([19], [21], [3] and [18]). The aim of this non-linear method is to approximate a function from its series expansion by constructing a fractional rational function such that its power series expansion agrees with that of the original function, insofar as possible. Such rational functions are called the Padé approximants and many generalizations of Padé approximants have been introduced over the past 80 years ([3], [47], [20], [64], [140], [41], [182] and [181]). The rational fractional approximants have found numerous applications in various branches of science and mechanical engineering because of their highly interesting approximation properties and, in particular, their potential convergence outside the domain of convergence of the series they approximate: they allow simplification of the non-linear terms and computation of an accurate approximation of a non-linear function $f(\mathbf{x})$, even at values of $f$ for which the Taylor series of $f(\mathbf{x})$ diverge.

There are other reduction, simplification and approximation methods used in non-linear dynamical analysis including the multiple shooting method, the Time Finite Element Method, the Multiple Scale Method, the fixed point procedure, etc ([185], [42][43], [24] and [97]).

We will now describe in detail three non-linear methods to reduce and simplify non-linear systems: the centre manifold, the normal forms and the fractional approximants. By applying these methods, we can reduce the dimension of the original system and/or simplify the number of non-linear terms without losing the non-linear behaviour of the original system. We do not claim to make an important contribution nor to be exhaustive; the only purpose is to give an overview of the non-linear methods that underlie this paper. We refer the interested reader to the books [134], [135], [3] and [70] for an extensive overview of the non-linear analysis and non-linear methods that are not the subject of this study. 


\subsection{The centre manifold approach}

In the field of engineering, the description of a dynamical system is usually given by a $n$-dimensional differential equation

$$
\dot{\mathbf{x}}=\mathbf{F}(\mathbf{x}, \mu)
$$

where $\mu$ is a control parameter and $\mathbf{F}$ is a non-linear function.

The centre manifold analysis is used to reduce the order of the non-linear model at near-equilibrium point $\mathbf{x}_{0}$ upon bifurcation ([135], [70], [99] and [82]). This approach is based on the idea that all the dynamical system characteristics at near-equilibrium point are governed by the dynamics on the centre manifold when some eigenvalues have zero real parts and all the other eigenvalues have negative real parts. To determine the centre manifold with a fixed point $\mathbf{x}_{\mathbf{0}}$ (defined by $\mathbf{f}\left(\mathbf{x}_{0} ; \mu=\mathbf{0}\right)$ ), the transformation $\mathbf{x}=\mathbf{x}_{\mathbf{0}}+\mathbf{y}$ is used to shift the fixed point $\mathbf{x}_{\mathbf{0}}$ of the system (1) to the origin. The non-linear system (1) is transformed by

$$
\dot{\mathbf{y}}=\mathbf{F}\left(\mathbf{y}+\mathbf{x}_{\mathbf{0}}, \mu\right)
$$

Assuming that the function $\mathbf{F}$ is at least $C^{2}$,the non-linear system (2) can be extanded in a Taylor series about $\mathbf{x}_{\mathbf{0}}$ for small $\|\mathbf{y}\|$ as follow

$$
\dot{\mathbf{y}}=\mathbf{A y}+\mathbf{F}_{\mathbf{2}}(\mathbf{y})+\mathbf{F}_{\mathbf{3}}(\mathbf{y})+\cdots+\mathbf{F}_{\mathbf{k}}(\mathbf{y})+O\left(\mathbf{y}^{\mathbf{k}+\mathbf{1}}\right)
$$

where $\mathbf{A}=D_{\mathbf{x}} f\left(\mathbf{x}_{\mathbf{0}} ; \mu\right)$ is the $n \times n$ matrix of first derivatives of $\mathbf{F}$ evaluated at the fixed point $\left(\mathbf{x}_{\mathbf{0}} ; \mu\right) . \mathbf{F}_{\mathbf{k}}$ is a degree $k$ polynomial series in the principal coordinates of degree $\mathbf{y}$.

Next, the linear transformation $\mathbf{y}=\mathbf{P v}$ where $\mathbf{P}=\left[\mathbf{p}_{\mathbf{1}} \cdots \mathbf{p}_{\mathbf{m}}, \mathbf{p}_{\mathbf{m}+\mathbf{1}} \cdots \mathbf{p}_{\mathbf{n}}\right]$ is introduced. $\mathbf{p}_{\mathbf{1}} \cdots \mathbf{p}_{\mathbf{m}}$ and $\mathbf{p}_{\mathbf{m}+\mathbf{1}} \cdots \mathbf{p}_{\mathbf{n}}$ are the generalized eigenvectors of the $m$ eigenvalues of $\mathbf{A}$ with zero real parts, and $(n-m)$ eigenvalues of $\mathbf{A}$ with nonzero real parts, respectively. The system (3) is transformed by

$$
\dot{\mathbf{v}}=\mathbf{J} \mathbf{v}+\mathbf{P}^{-1} \mathbf{F}_{\mathbf{2}}(\mathbf{P v})+\cdots+\mathbf{P}^{-1} \mathbf{F}_{\mathbf{k}}(\mathbf{P} \mathbf{v})+\cdots
$$

where $\mathbf{J}=\mathbf{P}^{-1} \mathbf{A P}$. At the Hopf bifurcation point, the system (4) may be rewritten in the form

$$
\left\{\begin{array}{l}
\dot{\mathbf{v}_{\mathbf{c}}}=\mathbf{J}_{\mathbf{c}}\left(\mu_{0}\right) \mathbf{v}_{\mathbf{c}}+\mathbf{F}_{\mathbf{c}}\left(\mathbf{v}_{\mathbf{c}}, \mathbf{v}_{\mathbf{s}}, \mu_{0}\right)=\mathbf{J}_{\mathbf{c}} \mathbf{v}_{\mathbf{c}}+\mathbf{G}_{\mathbf{2}}\left(\mathbf{v}_{\mathbf{c}}, \mathbf{v}_{\mathbf{s}}, \mu_{0}\right)+\cdots+\mathbf{G}_{\mathbf{k}}\left(\mathbf{v}_{\mathbf{c}}, \mathbf{v}_{\mathbf{s}}, \mu_{0}\right)+\cdots \\
\dot{\mathbf{v}_{\mathbf{s}}}=\mathbf{J}_{\mathbf{s}}\left(\mu_{0}\right) \mathbf{v}_{\mathbf{s}}+\mathbf{F}_{\mathbf{s}}\left(\mathbf{v}_{\mathbf{c}}, \mathbf{v}_{\mathbf{s}}, \mu_{0}\right)=\mathbf{J}_{\mathbf{s}} \mathbf{v}_{\mathbf{s}}+\mathbf{H}_{\mathbf{2}}\left(\mathbf{v}_{\mathbf{c}}, \mathbf{v}_{\mathbf{s}}, \mu_{0}\right)+\cdots+\mathbf{H}_{\mathbf{k}}\left(\mathbf{v}_{\mathbf{c}}, \mathbf{v}_{\mathbf{s}}, \mu_{0}\right)+\cdots
\end{array}\right.
$$

where $\mathbf{v}_{\mathbf{c}} \in \Re^{n}$ and $\mathbf{v}_{\mathbf{s}} \in \Re^{m-n}$. $\mathbf{J}_{\mathbf{c}}$ and $\mathbf{J}_{\mathbf{s}}$ have eigenvalues $\lambda$ such that $\operatorname{Re}\left(\lambda_{\mathbf{J}_{\mathbf{c}}}\left(\mu_{0}\right)\right)=0$ and $\operatorname{Re}\left(\lambda_{\mathbf{J}_{\mathbf{s}}}\left(\mu_{0}\right)\right) \neq 0$ where $\mu_{0}$ is the value of the control parameter at the Hopf bifurcation point. It may be noted that $\mathbf{v}_{\mathbf{c}}$ and $\mathbf{v}_{\mathbf{s}}$ are linearly uncoupled but non-linearly coupled. Moreover, $\mathbf{G}_{\mathbf{i}}(\mathbf{0}, \mathbf{0})=\mathbf{0}, \mathbf{H}_{\mathbf{i}}(\mathbf{0}, \mathbf{0})=\mathbf{0}$, and the Jacobian matrices $D \mathbf{G}_{\mathbf{i}}(\mathbf{0}, \mathbf{0})$ and $D \mathbf{H}_{\mathbf{i}}(\mathbf{0}, \mathbf{0})$ are matrices with zero entries (with $1<i \leq k$ ).

Now, a simple extension to the centre manifold method ([116], [135] and [70]) which is useful when dealing with parametrized systems may be defined by augmenting the original system (5)

$$
\left\{\begin{array}{l}
\dot{\mathbf{v}_{\mathbf{c}}}=\mathbf{J}_{\mathbf{c}}(\hat{\mu}) \mathbf{v}_{\mathbf{c}}+\mathbf{F}_{\mathbf{c}}\left(\mathbf{v}_{\mathbf{c}}, \mathbf{v}_{\mathbf{s}}, \hat{\mu}\right) \\
\dot{\mathbf{v}_{\mathbf{s}}}=\mathbf{J}_{\mathbf{s}}(\hat{\mu}) \mathbf{v}_{\mathbf{s}}+\mathbf{F}_{\mathbf{s}}\left(\mathbf{v}_{\mathbf{c}}, \mathbf{v}_{\mathbf{s}}, \hat{\mu}\right) \\
\dot{\hat{\mu}}=0
\end{array}\right.
$$

where $\hat{\mu}$ defines the modification of $\mu_{0}$ around the Hopf bifurcation point. $\quad \mathbf{F}_{\mathbf{c}}$ and $\mathbf{F}_{\mathbf{s}}$ are polynomial non-linear functions in the components of $\mathbf{v}_{\mathbf{c}}$ and $\mathbf{v}_{\mathbf{s}}$ and infinitely differential. At $\left(\mathbf{v}_{\mathbf{c}}, \mathbf{v}_{\mathbf{s}}, \hat{\mu}\right)=(\mathbf{0}, \mathbf{0}, 0)$, the center space is $\left(\mathbf{v}_{\mathbf{c}}, \hat{\mu}\right)$. For only small $\left\|\mathbf{v}_{\mathbf{c}}\right\|$ and $\|\hat{\mu}\|$, a local centre manifold exists and the centre manifold theory (Carr [28]) allows the expression of the stables variables $\mathbf{v}_{\mathbf{s}}$ as a function of the center variables $\left(\mathbf{v}_{\mathbf{c}}, \hat{\mu}\right)$ such that

$$
\mathbf{v}_{\mathbf{s}}=\mathbf{h}\left(\mathbf{v}_{\mathbf{c}}, \hat{\mu}\right)
$$

where the function $\mathbf{h}$ verified at the fixed point $(\mathbf{0}, \mathbf{0}, 0)$

$$
\begin{gathered}
\mathbf{h}=\mathbf{0} \\
D_{\mathbf{v}_{\mathbf{c}}} h_{i}(\mathbf{0})=\mathbf{0} \quad 1 \leq i \leq n-2
\end{gathered}
$$




$$
\frac{\partial \mathbf{h}}{\partial \hat{\mu}}=0
$$

where $h_{i}$ are the scalar components of $\mathbf{h}$. Due to the fact that the expression of $\mathbf{h}$ cannot be solved exactly in most cases, the stable variables are usually approximated as a power series in $\left(\mathbf{v}_{\mathbf{c}}, \hat{\mu}\right)$ of degree $m$

$$
\mathbf{v}_{\mathbf{s}}=\mathbf{h}\left(\mathbf{v}_{\mathbf{c}}, \hat{\mu}\right)=\sum_{p=i+j+\cdots+k+l=2}^{m} \sum_{j=0}^{p} \cdots \sum_{k=0}^{p} \sum_{l=0}^{p} \mathbf{a}_{i j \cdots k l} v_{c_{1}}^{i} v_{c_{2}}^{j} \cdots v_{c_{n}}^{k} \hat{\mu}^{l}
$$

where $\mathbf{a}_{i j \cdots k l}$ are vectors of constant coefficients. It may be noted that $\mathbf{h}$ is a power series without constant and linear terms in order to satisfy equations (8-10). The choice of an approximation of the stable variables $\mathbf{v}_{\mathbf{s}}$ as a power series in the centre variables $\mathbf{v}_{\mathbf{c}}$ is not an obligation; however, that makes it possible to check easily the conditions defined in equations (8-10). The determination of the vector $\mathbf{a}_{i j \ldots k l}$ is obtained by substituting the $(m-n)$ dimensional function $\mathbf{h}$ into the second equation of (6). By combinaison with the first equation of (6), the function $\mathbf{h}$ verified

$$
D_{\mathbf{v}_{\mathbf{c}}, \hat{\mu}}\left(\mathbf{h}\left(\mathbf{v}_{\mathbf{c}}, \hat{\mu}\right)\right)\left(\mathbf{J}_{\mathbf{c}} \mathbf{v}_{\mathbf{c}}+\mathbf{F}_{\mathbf{c}}\left(\mathbf{v}_{\mathbf{c}}, \mathbf{h}\left(\mathbf{v}_{\mathbf{c}}, \hat{\mu}\right), \hat{\mu}\right)\right)=\mathbf{J}_{\mathbf{s}} \mathbf{h}\left(\mathbf{v}_{\mathbf{c}}, \hat{\mu}\right)+\mathbf{F}_{\mathbf{s}}\left(\mathbf{v}_{\mathbf{c}}, \mathbf{h}\left(\mathbf{v}_{\mathbf{c}}, \hat{\mu}\right), \hat{\mu}\right)
$$

To solve equation (12), we equate the coefficients of the different terms in the polynomials on both sides and we obtain a system of algebraic equations for the coefficients $\mathbf{a}_{i j \cdots k l}$ of the polynomials. By solving these equations, we obtain an approximation to the centre manifold $\mathbf{v}_{\mathbf{s}}=\mathbf{h}\left(\mathbf{v}_{\mathbf{c}}, \hat{\mu}\right)$. After $\mathbf{h}$ is identified, the reduced order structural dynamic model, which is only a function of $\mathbf{v}_{\mathbf{c}}$, is given by

$$
\left\{\begin{array}{l}
\dot{\mathbf{v}}_{\mathbf{c}}=\mathbf{J}_{\mathbf{c}}(\hat{\mu}) \mathbf{v}_{\mathbf{c}}+\mathbf{F}_{\mathbf{c}}\left(\mathbf{v}_{\mathbf{c}}, \mathbf{h}\left(\mathbf{v}_{\mathbf{c}}, \hat{\mu}\right), \hat{\mu}\right) \\
\dot{\hat{\mu}}=0
\end{array}\right.
$$

If $n$ of the $m$ eigenvalues have zero real parts, then we reduce the number of equations of the original system from $m$ to $n$ in order to obtain a simplified system. In the field of mechanic engineering, the centre manifold is frequently composed of only two centre variables so that the reduced non-linear system (13) contains only two degrees-offreedom with a control parameter $\hat{\mu}$, called an unfolding parameter.

\subsection{The Normal forms simplification}

As explained previously, the centre manifold reduces the non-linear system on the centre variables. However, the associated centre manifold equations can have complicated non-linear terms due to the approximation of the stable variables in a power series in centre variables and their substitutions in the centre manifold equations. For such systems, non-linear successive coordinate transformations can be used to reduce this non-linear system to its simplest form, called the normal form. The idea of the normal transformation comes from Poincare ([145] and [146]) and this normal forms theory has been studied by several groups ([103], [2], [125], [133], [7], [34], [22], [23], [92], [54], [93], [83], [126] and [171]). The main idea of the normal form approach is to eliminate as many non-linear terms as possible through a non-linear change of variables. This normal forms theory is a very powerful tool for the analysis of the local dynamical behavior near a singularity ([72], [203], [6], [204], and [206]).

\subsubsection{Normal form theory}

Consider the nonlinear system described by

$$
\dot{x}_{i}=f_{i}(\mathbf{x}) \quad i=1, \ldots, n
$$

where each $f_{i}$ is a function of $\mathbf{x}$. By introducing the formal invertible transformation

$$
x_{i}=\xi_{i}(\mathbf{y})=y_{i} \sum_{\mathbf{q} \in N_{i}} h_{i \mathbf{q}} \mathbf{y}^{\mathbf{q}} \quad i=1, \ldots, n
$$

every system (14) may be transformed retaining the Jordan form of the system (14) in the normal form defined by

$$
\dot{y}_{i}=y_{i} \sum_{\mathbf{q} \cdot \Lambda=0} g_{i \mathbf{q}} \mathbf{y}^{\mathbf{q}} \quad i=1, \ldots, n
$$


where

$$
\begin{gathered}
\mathbf{y}^{\mathbf{q}}=\left(y_{1}^{q_{1}}, y_{2}^{q_{2}}, \cdots, y_{n}^{q_{n}}\right) \\
N_{i}=\left\{\mathbf{q} \text { integral vector }: q_{i} \geq-1, \quad q_{k} \geq 0 \quad \text { if } \quad k \neq i, \quad \sum_{i=1}^{n} q_{i}=0\right\} \\
g_{i \mathbf{q}}=0 \quad \text { if } \mathbf{q} \cdot \Lambda=\sum_{i=1}^{n} q_{i} \lambda_{i} \neq 0 \\
\Lambda=\left\{\lambda_{1}, \lambda_{2}, \ldots, \lambda_{n}\right\}^{T}
\end{gathered}
$$

where $\Lambda$ defines the vector of the linear eigenvalues of the non-linear system (14). Due to the relation (20), it may be observed that the right side of the expression (16) contains only the resonant terms $y_{i} g_{i \mathbf{q}} \mathbf{y}^{\mathbf{q}}$ verifying $\mathbf{q} \cdot \Lambda=$ $\sum_{i=1}^{n} q_{i} \lambda_{i}=0$.

\subsubsection{Transformation to normal form}

In this section, it is proposed to compute the normal form transformation by using a successive change of variables according to the increasing degrees of non-linearities ([70], [92] and [206]). Effectively, as explained previously, the basic idea of the method of normal forms is to employ successive coordinate transformation to systematically construct a simple form of the original non-linear system.

By considering the previous system (14), we expand the vector field $\mathbf{f}(\mathbf{x})$ into a Taylor series

$$
\dot{\mathbf{x}}=\mathbf{f}(\mathbf{x})=\mathbf{A} \mathbf{x}+\mathbf{f}^{2}(\mathbf{x})+\mathbf{f}^{3}(\mathbf{x})+\cdots+\mathbf{f}^{N}(\mathbf{x})+O\left(|\mathbf{x}|^{N+1}\right)=\mathbf{A} \mathbf{x}+\sum_{k=2}^{N} \mathbf{f}^{k}(\mathbf{x})+O\left(|\mathbf{x}|^{N+1}\right)
$$

where $\mathbf{x} \in \Re^{n}$. The matrix $\mathbf{A}$ may be assumed to be in the Jordan standard form; if this is not the case, $\mathbf{A}$ may be changed easily to block Jordan form by a linear transformation. We have $\mathbf{f}^{k} \in H_{n}^{k}$ where $H_{n}^{k}$ defines the $n$-variate polynomial space of order $k$ (with $k \geq 2$ ).

Introducing the non-linear transformations

$$
\begin{array}{lcc}
\mathbf{x}=\mathbf{y}_{\mathbf{2}}+\mathbf{P}^{2}\left(\mathbf{y}_{\mathbf{2}}\right) \quad \text { with } \quad \mathbf{P}^{2}\left(\mathbf{y}_{\mathbf{2}}\right) \in H_{n}^{2} \\
\mathbf{y}_{\mathbf{2}}=\mathbf{y}_{\mathbf{3}}+\mathbf{P}^{3}\left(\mathbf{y}_{\mathbf{3}}\right) \quad \text { with } \quad \mathbf{P}^{3}\left(\mathbf{y}_{\mathbf{3}}\right) \in H_{n}^{3} \\
\vdots \\
\mathbf{y}_{\mathbf{k}-\mathbf{1}}=\mathbf{y}_{\mathbf{k}}+\mathbf{P}^{k}\left(\mathbf{y}_{\mathbf{k}}\right) \quad \text { with } & \mathbf{P}^{k}\left(\mathbf{y}_{\mathbf{k}}\right) \in H_{n}^{k} \\
\vdots & & \\
\mathbf{y}_{\mathbf{N}-\mathbf{1}}=\mathbf{y}_{\mathbf{N}}+\mathbf{P}^{N}\left(\mathbf{y}_{\mathbf{N}}\right) \quad \text { with } & \mathbf{P}^{N}\left(\mathbf{y}_{\mathbf{N}}\right) \in H_{n}^{N}
\end{array}
$$

and substituting these expressions in the non-linear system (21), the left side is

$$
\begin{aligned}
& \dot{\mathbf{x}}=\frac{d}{d t}\left(\mathbf{y}_{\mathbf{2}}+\mathbf{P}^{2}\left(\mathbf{y}_{\mathbf{2}}\right)\right)=\left(\mathbf{I}+D_{\mathbf{y}_{\mathbf{2}}} \mathbf{P}^{2}\left(\mathbf{y}_{\mathbf{2}}\right)\right) \dot{\mathbf{y}_{\mathbf{2}}} \\
& \dot{\mathbf{y}_{\mathbf{2}}}=\frac{d}{d t}\left(\mathbf{y}_{\mathbf{3}}+\mathbf{P}^{3}\left(\mathbf{y}_{\mathbf{3}}\right)\right)=\left(\mathbf{I}+D_{\mathbf{y}_{\mathbf{3}}} \mathbf{P}^{3}\left(\mathbf{y}_{\mathbf{3}}\right)\right) \dot{\mathbf{y}_{\mathbf{3}}} \\
& \vdots \\
& \dot{\mathbf{y}_{\mathbf{k}-\mathbf{1}}}=\frac{d}{d t}\left(\mathbf{y}_{\mathbf{k}}+\mathbf{P}^{k}\left(\mathbf{y}_{\mathbf{k}}\right)\right)=\left(\mathbf{I}+D_{\mathbf{y}_{\mathbf{k}}} \mathbf{P}^{k}\left(\mathbf{y}_{\mathbf{k}}\right)\right) \dot{\mathbf{y}_{\mathbf{k}}} \\
& \vdots \\
& \mathbf{y}_{\mathbf{N}-\mathbf{1}}=\frac{d}{d t}\left(\mathbf{y}_{\mathbf{N}}+\mathbf{P}^{N}\left(\mathbf{y}_{\mathbf{N}}\right)\right)=\left(\mathbf{I}+D_{\mathbf{y}_{\mathbf{N}}} \mathbf{P}^{N}\left(\mathbf{y}_{\mathbf{N}}\right)\right) \dot{\mathbf{y}_{\mathbf{N}}}
\end{aligned}
$$


and the right side is

$$
\begin{aligned}
& \mathbf{f}(\mathbf{x})=\mathbf{A}\left(\mathbf{y}_{\mathbf{2}}+\mathbf{P}^{2}\left(\mathbf{y}_{\mathbf{2}}\right)\right)+\cdots+\mathbf{f}^{N}\left(\mathbf{y}_{\mathbf{2}}+\mathbf{P}^{2}\left(\mathbf{y}_{\mathbf{2}}\right)\right)+O\left(|\mathbf{x}|^{N+1}\right) \\
& \mathbf{f}\left(\mathbf{y}_{\mathbf{2}}\right)=\mathbf{A}\left(\mathbf{y}_{\mathbf{3}}+\mathbf{P}^{3}\left(\mathbf{y}_{\mathbf{3}}\right)\right)+\cdots+\mathbf{f}_{2}^{N}\left(\mathbf{y}_{\mathbf{3}}+\mathbf{P}^{3}\left(\mathbf{y}_{\mathbf{3}}\right)\right)+O\left(|\mathbf{x}|^{N+1}\right) \\
& \vdots \\
& \mathbf{f}\left(\mathbf{y}_{\mathbf{k}-\mathbf{1}}\right)=\mathbf{A}\left(\mathbf{y}_{\mathbf{k}}+\mathbf{P}^{k}\left(\mathbf{y}_{\mathbf{k}}\right)\right)+\cdots+\mathbf{f}_{k-1}^{N}\left(\mathbf{y}_{\mathbf{k}}+\mathbf{P}^{k}\left(\mathbf{y}_{\mathbf{k}}\right)\right)+O\left(|\mathbf{x}|^{N+1}\right) \\
& \vdots \\
& \mathbf{f}\left(\mathbf{y}_{\mathbf{N}-\mathbf{1}}\right)=\mathbf{A}\left(\mathbf{y}_{\mathbf{N}}+\mathbf{P}^{N}\left(\mathbf{y}_{\mathbf{N}}\right)\right)+\cdots+\mathbf{f}_{N-1}^{N}\left(\mathbf{y}_{\mathbf{N}}+\mathbf{P}^{N}\left(\mathbf{y}_{\mathbf{N}}\right)\right)+O\left(|\mathbf{x}|^{N+1}\right)
\end{aligned}
$$

By identifying (23) and (24), we obtain for the $k^{\text {th }}$-transformation

$$
\dot{\mathbf{y}_{\mathbf{k}}}=\left(\mathbf{I}+D_{\mathbf{y}_{\mathbf{k}}} \mathbf{P}^{k}\left(\mathbf{y}_{\mathbf{k}}\right)\right)^{-1}\left(\mathbf{A y}_{\mathbf{k}}+\mathbf{A} \mathbf{P}^{k}\left(\mathbf{y}_{\mathbf{k}}\right)+\sum_{j=1}^{k} \sum_{i=2}^{N} \frac{D^{j} \mathbf{f}_{k-2}^{i}}{j !}\left(\mathbf{P}^{k}\left(\mathbf{y}_{\mathbf{k}}\right)\right)^{j}\right)
$$

Finally, after a number of computations [70], the reduced system for each $k^{\text {th }}$-normal form is obtained successively by

$$
\begin{aligned}
& \dot{\mathbf{y}_{\mathbf{2}}}=\mathbf{A} \mathbf{y}_{\mathbf{2}}+\mathbf{f}_{1}^{2}\left(\mathbf{y}_{\mathbf{2}}\right)+\sum_{i=3}^{N} \mathbf{f}_{1}^{i}\left(\mathbf{y}_{\mathbf{2}}\right) \\
& \dot{\mathbf{y}_{3}}=\mathbf{A} \mathbf{y}_{\mathbf{3}}+\sum_{i=2}^{3} \mathbf{f}_{i-1}^{i}\left(\mathbf{y}_{\mathbf{3}}\right)+\sum_{i=4}^{N} \mathbf{f}_{2}^{i}\left(\mathbf{y}_{\mathbf{3}}\right) \\
& \vdots \\
& \dot{\mathbf{y}_{\mathbf{k}}}=\mathbf{A} \mathbf{y}_{\mathbf{k}}+\sum_{i=2}^{k} \mathbf{f}_{i-1}^{i}\left(\mathbf{y}_{\mathbf{k}}\right)+\sum_{i=k+1}^{N} \mathbf{f}_{k-1}^{i}\left(\mathbf{y}_{\mathbf{k}}\right) \\
& \vdots \\
& \dot{\mathbf{y}_{\mathbf{N}}}=\mathbf{A y}_{\mathbf{N}}+\sum_{i=2}^{N} \mathbf{f}_{i-1}^{i}\left(\mathbf{y}_{\mathbf{N}}\right)
\end{aligned}
$$

As illustrated in the previous equations, the normal form transformations absorb lower degree non-linearities inside the coordinate definitions while generating higher degree non-linearities. These coordinate transformations are generally a good computational tool because by applying transformations, we simplify the original non-linear expression without losing the mathematical and physical properties of systems. It may be noted that the normal forms are generally not uniquely defined and finding a normal form of non-linear systems is not easy. However, all the expressions of $\mathbf{f}_{j}^{i}$ and the related coefficients can be obtained by using symbolic calculations and solving successively a series of algebraic equations according to the increasing degrees of non-linearities ([70] and [92]). The normal forms method greatly simplifies the analysis of dynamic behaviour of the original non-linear system. However, this procedure may be not very convenient, and other procedures have been developed to more easily obtain the normal form of a non-linear $\operatorname{system}([203],[6],[147],[206]$, [73] and [72]).

\subsection{The Padé and multivariable approximants}

As shown in Sections 2.2 and 2.3, the centre manifold equations can have complicated non-linear terms. These nonlinear tems can be simplified by using further non-linear methods, such as the normal forms approach. One of the other most interesting possibilities is to apply the Padé approximants and the general rational fractional approximants ([19], [21], [3] and [18]). As explained previously, the interest of the Padé approximants and general rational fractional approximants is that they need fewer terms than the associated Taylor series in order to obtain an accurate approximation of a solution of a given problem. The aim of this section is to provide an introduction to Padé approximations and the general rational fractional approximants in 2-variables that will be applied after the centre manifold analysis. In many branches of engineering sciences, Padé approximants and rational fractional approximants have found numerous applications ([40], [35], [36], [201], [200], [55], [155],[170], [169], [168] and [175]); the solution of a given 
problem in applied sciences is often described as a power series expansion. In this case, one of the interesting aims is to approximate the function from its series expansion. Such constructions of rational functions are called the Padé approximants and general fraction approximants. They have quite interesting approximation properties and possible convergence outside the domain of convergence of the series they approximate; a sequence of rational fractional approximants may converge even if the associated series does not, so that the domain of convergence may be extended by employing rational fractional approximants [174].

\subsubsection{Padé approximants}

Let the function $f(z)$ be represented by a formal power series, so that

$$
f(z)=\sum_{i=0}^{\infty} c_{i} z^{i}
$$

A Padé approximant $g(z)$ is a rational approximation of the function $f(z)$ such that its power series expansion matches the formal power series $f(z)$ insofar as possible. The Padé approximant $g(z)$ associated with the formal power series $f(z)=\sum_{i=0}^{\infty} c_{i} z^{i}$ is the rational function

$$
g(z)=\frac{a_{0}+a_{1} z+\cdots+a_{m} z^{m}}{b_{0}+b_{1} z+\cdots+b_{n} z^{n}}
$$

such that

$$
f(z)-g(z)=O\left(z^{m+n+1}\right)
$$

The Padé approximant $g(z)$ with a numerator of degree $L$ at most, and a denominator of the degree $n$ at most is usually denoted by

$$
[m / n]_{f}(z)
$$

Next, the aim is to determine the unknown coefficients $\left(a_{0}, a_{1}, \cdots, a_{m}\right)$ and $\left(b_{0}, b_{1}, \cdots, b_{n}\right)$. There are $m+1$ unknown numerator coefficients and $n+1$ unknown denominator coefficients. By considering the equation (28), we note that the coefficients $\left(a_{0}, \cdots, a_{m}\right)$ and $\left(b_{0}, \cdots, b_{n}\right)$ will be determined at most up to a common multiplicative factor. So, for definiteness we assume that $b_{0}=1$. So there are $m+n+1$ unknown independent coefficients in all $(m+1$ unknown independent numerator coefficients and $n$ unknown independent denominator coefficients).

By multiplying the difference between $f(z)$ and $[m / n]_{f}(z)$ by the denominator of $[m / n]_{f}(z)$, we find that

$$
\left(b_{0}+b_{1} z+\cdots+b_{n} z^{n}\right)\left(c_{0}+c_{1} z+\cdots\right)=a_{0}+a_{1} z+\cdots+a_{m} z^{m}+O\left(z^{m+n+1}\right)
$$

By equating the coefficients of $z^{m+1}, z^{m+2}, \cdots, z^{m+n}$, we obtain

$$
\left\{\begin{array}{l}
b_{0} c_{m+1}+\cdots+b_{n-1} c_{m-n+2}+b_{n} c_{m-n+1}=0 \\
b_{0} c_{m+2}+\cdots+b_{n-1} c_{m-n+3}+b_{n} c_{m-n+2}=0 \\
\cdots \\
b_{0} c_{m+n}+\cdots+b_{n-1} c_{m+1}+b_{n} c_{m}=0 \\
\text { with } \quad c_{i}=0 \quad \text { if } \quad i<0
\end{array}\right.
$$

It may be noted that the system (32) is a set of $n$ linear equations for the $n$ unknown denominator coefficients $\left(b_{1}, \cdots, b_{n}\right)$ (by assuming $b_{0}=1$ ). Then, the $m+1$ unknown coefficients $\left(a_{0}, a_{1}, \cdots, a_{m}\right)$ can be obtained directly from the equation (31) by equating the coefficients of $z^{1}, z^{2}, \cdots, z^{m}$

$$
\left\{\begin{array}{l}
a_{0}=c_{0} b_{0} \\
a_{1}=c_{1} b_{0}+c_{0} b_{1} \\
a_{2}=c_{2} b_{0}+c_{1} b_{1}+c_{0} b_{2} \\
\cdots \\
a_{m}=c_{m} b_{0}+\sum_{i=1}^{\min (m, n)} b_{i} c_{m-i}
\end{array}\right.
$$




\subsubsection{The multivariable rational fractional approximants}

A natural problem in applied sciences is the generalisation of the Padé approximants to more than one variable. In this section, we limit our attention to the case of the rational polynomial approximants in two variables that will be studied in this paper. All the problems associated with many variables have the same kind of solutions and the determination of the numerator and denominator coefficients of the fractional approximants follows the same steps [75].

Let $f(x, y)$ be a function of two variables of the form

$$
f(x, y)=\sum_{\alpha=0}^{\infty} \sum_{\beta=0}^{\infty} c_{\alpha \beta} x^{\alpha} y^{\beta}=\sum_{(\alpha, \beta) \in S} c_{\alpha \beta} x^{\alpha} y^{\beta}
$$

where

$$
S=\{(\alpha, \beta) \mid \alpha, \beta \quad \text { nonnegative integers }\}
$$

We define

$$
A(x, y)=\sum_{(\alpha, \beta) \in S_{m}} a_{\alpha \beta} x^{\alpha} y^{\beta}
$$

and

$$
B(x, y)=\sum_{(\alpha, \beta) \in S_{n}} b_{\alpha \beta} x^{\alpha} y^{\beta}
$$

such that

$$
f(x, y)=\frac{A(x, y)}{B(x, y)}+\sum_{\alpha=0}^{\infty} \sum_{\beta=0}^{\infty} d_{\alpha \beta} x^{\alpha} y^{\beta}
$$

where as many coefficients $d_{\alpha \beta}$ as possible are zero and with $S_{m}$ et $S_{n}$ defined as follows

$$
\begin{gathered}
S_{m}=\left\{(\alpha, \beta) \mid 0 \leq \alpha \leq m_{1}, 0 \leq \beta \leq m_{2}\right\} \\
S_{n}=\left\{(\alpha, \beta) \mid 0 \leq \alpha \leq n_{1}, 0 \leq \beta \leq n_{2}\right\}
\end{gathered}
$$

In this case, $\frac{A(x, y)}{B(x, y)}$ is the rational fractional polynomial approximants in two-variables of $f(x, y)$. It has the form

$$
[m / n]_{f}(x, y)=\frac{\sum_{(\alpha, \beta) \in S_{m}} a_{\alpha \beta} x^{\alpha} y^{\beta}}{\sum_{(\alpha, \beta) \in S_{n}} b_{\alpha \beta} x^{\alpha} y^{\beta}}=\left[m_{1}, m_{2} / n_{1}, n_{2}\right]_{f}(x, y)=\frac{\sum_{\alpha=0}^{m_{1}} \sum_{\beta=0}^{m_{2}} a_{\alpha \beta} x^{\alpha} y^{\beta}}{\sum_{\alpha=0}^{n_{1}} \sum_{\beta=0}^{n_{2}} b_{\alpha \beta} x^{\alpha} y^{\beta}}
$$

By assuming that $b_{00}=0$, there are $\left(m_{1}+1\right)\left(m_{2}+1\right)+n_{1}\left(n_{2}+1\right)$ unknown coefficients to be found in the fractional approximant (41). In order to understand the procedure to determine the unknown coefficients and to illustrate the various types of regions in which the terms $x^{\alpha} y^{\beta}$ are to be matched, it is useful to consider a few diagrams in the two-dimensional lattice set $S$, as illustrated in Figures 1-3. Figures 1, 2 and 3 illustrate the situation for a Chisholm Approximant $\left(m_{1}=m_{2}=n_{1}=n_{2}=m\right)$, a Simple-Off-Diagonal approximant $\left(m_{1}=m_{2}=m\right.$ and $\left.n_{1}=n_{2}=n\right)$, and a General-Off-Diagonal approximant $\left(m_{1} \neq m_{2} \neq n_{1} \neq n_{2}\right)$, respectively.

We use the following convenient notation:

$$
\begin{gathered}
m_{i}^{\prime}=\min \left(m_{i}, n_{i}\right) \\
n_{i}^{\prime}=\max \left(m_{i}, n_{i}\right) \\
p_{m}=\min \left(m_{1}, m_{2}, n_{1}, n_{2}\right)
\end{gathered}
$$

and the following sets

$$
\begin{gathered}
P=\left\{(p, p) \mid 0 \leq p \leq p_{m}\right\} \\
R_{1 ; p}=\{(p, p)\} \cup\left\{(\alpha, p) \mid p<\alpha \leq m_{1}^{\prime}\right\} \cup\left\{(p, \beta) \mid p<\beta \leq m_{2}^{\prime}\right\} \\
R_{2 ; p}=\left\{(\alpha, p) \mid m_{1}^{\prime}<\alpha \leq n_{1}^{\prime}\right\} \cup\left\{(p, \beta) \mid m_{2}^{\prime}<\beta \leq n_{2}^{\prime}\right\}
\end{gathered}
$$




$$
\begin{gathered}
R_{3 ; p}=\left\{(\alpha, p) \mid n_{1}^{\prime}<\alpha \leq m_{1}+n_{1}-p\right\} \cup\left\{(p, \beta) \mid n_{2}^{\prime}<\beta \leq m_{2}+n_{2}-p\right\} \\
R_{4 ; p}=\left\{\left(m_{1}+n_{1}-p+1, p\right),\left(p, m_{2}+n_{2}-p+1\right)\right\}
\end{gathered}
$$

Finally, the various types of regions indicated in Figures 1-3 are defined as follows

$$
\begin{gathered}
S_{1}=S_{m} \cap S_{n} \\
S_{2}=S_{m} \cup S_{n} \backslash S_{1} \\
S_{3}=\bigcup_{p \in P} R_{3 ; p} \\
S_{4}=\bigcup_{p \in P} R_{4 ; p} \\
S_{5}=S_{2} \backslash\left(\bigcup_{p \in P} R_{2 ; p}\right)
\end{gathered}
$$

Next, by considering equations (38) and (41), and by multiplying the difference between $f(x, y)$ and $[m / n]_{f}(x, y)$ by the denominator of $[m / n]_{f}(x, y)$, we obtain

$$
\left(\sum_{(\alpha, \beta) \in S_{n}} b_{\alpha \beta} x^{\alpha} y^{\beta}\right) \cdot\left(\sum_{(\alpha, \beta) \in S} c_{\alpha \beta} x^{\alpha} y^{\beta}\right)-\sum_{(\alpha, \beta) \in S_{m}} a_{\alpha \beta} x^{\alpha} y^{\beta}=\sum_{(\alpha, \beta) \in S} d_{\alpha \beta} x^{\alpha} y^{\beta}
$$

with

$$
d_{\alpha \beta}=0 \quad(\alpha, \beta) \in R_{1 ; p} \cup R_{2 ; p} \cup R_{3 ; p}
$$

and

$$
\sum_{p \neq 0} d_{m_{1}+n_{1}-p+1, p}+d_{p, m_{2}+n_{2}-p+1}=0
$$

Finally, by matching the coefficients for identical power of $x^{\alpha} y^{\beta}$ in (55), the following relations are obtained

$$
\begin{gathered}
\sum_{\psi \in S_{n}} b_{\psi} c_{\rho-\psi}=a_{\rho} \quad \rho \in S_{m} \\
\sum_{\psi \in S_{n}} b_{\psi} c_{\rho-\psi}=0 \quad \psi \in\left(S_{n} \backslash S_{m}\right) \cup S_{3} \\
\sum_{\psi \in R_{4 ; p}} \sum_{\rho \in S_{n}} b_{\psi} c_{\rho-\psi}=0 \quad p \in P
\end{gathered}
$$

where $c_{\alpha \beta}=0$ if either $\alpha$ or $\beta$ is negative. Then, after normalising $b_{00}$ to unity, the computation of the coefficients $b_{\psi}$ can be achieved by solving the linear equations which arise from (59) and (60). Next, the linear equations given by equations (58) enable the coefficients $a_{\rho}$ to be determined, with the coefficients $b_{\psi}$ found previously.

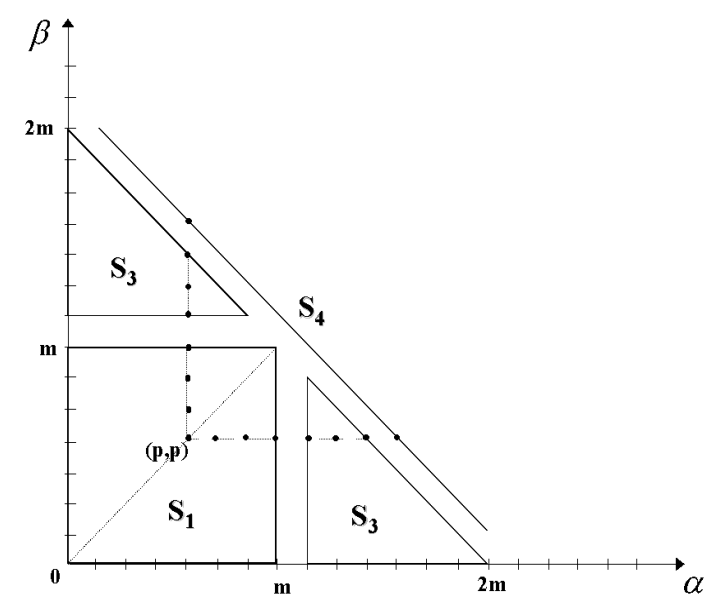

Figure 1: Various types of regions for the Chisholm Approximants (CA) of two variables 


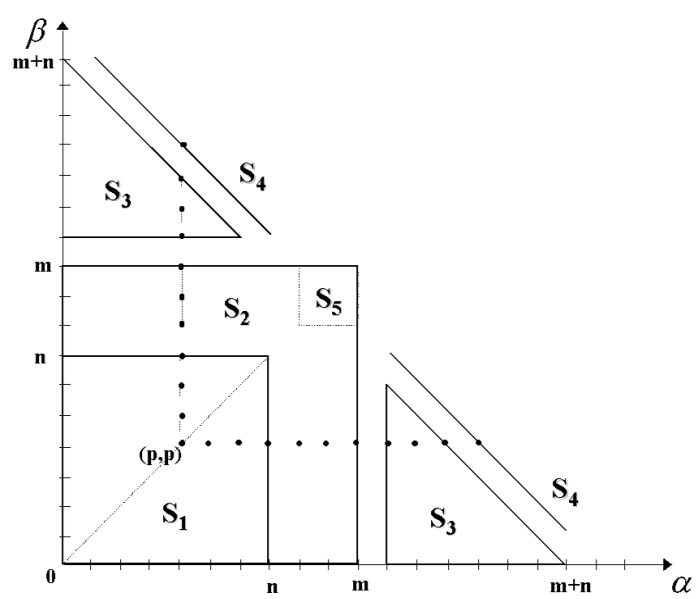

Figure 2: Various types of regions for the Symmetric-Off-Diagonal (SOD) approximants of two variables

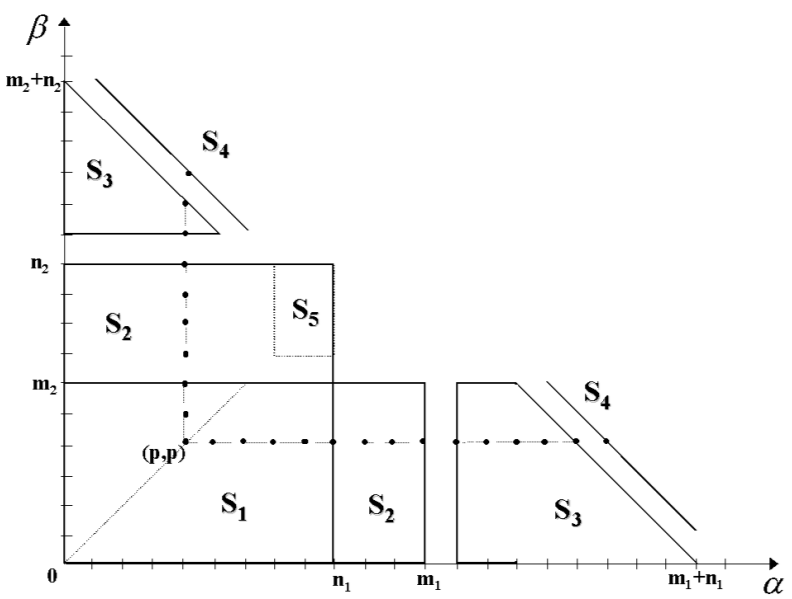

Figure 3: Various types of regions for the General-Off-Diagonal (GOD) approximants of two variables

\subsubsection{General fractional approximants in $k$-variables}

It may be observed that the generalisation for the rational fractional aproximants in $k$-variables is a fairly natural process. Let $f\left(x_{1}, x_{2}, \ldots, x_{k}\right)$ be a function of $k$-variables defined by a formal power series expansion

$$
f\left(x_{1}, x_{2}, \ldots, x_{k}\right)=\sum_{\alpha_{1}=0}^{\infty} \cdots \sum_{\alpha_{k}=0}^{\infty} c_{\alpha_{1} \ldots \alpha_{k}} x_{1}^{\alpha_{1}} \cdots x_{k}^{\alpha_{k}}
$$

with

$$
\begin{gathered}
\mathbf{x}=\left(x_{1}, \ldots, x_{k}\right) \\
\alpha=\left(\alpha_{1}, \ldots, \alpha_{k}\right) \\
\mathbf{x}^{\alpha} \equiv x_{1}^{\alpha_{1}} \cdots x_{k}^{\alpha_{k}} \\
I_{k}=\{1, \ldots k\} \\
S=\left\{\alpha \mid \alpha_{i} \in \aleph^{+}, \quad i \in I_{k}\right\}
\end{gathered}
$$

The power series defined in (61) with $k$-variables has the compact form

$$
f(\mathbf{x})=\sum_{\alpha \in S} c_{\alpha} \mathbf{x}^{\alpha}
$$


The associated rational approximants in $k$-variables are given by

$$
[m / n]_{f}(\mathbf{x})=\frac{\sum_{\mu \in S_{m}} a_{\mu} \mathbf{x}^{\mu}}{\sum_{\sigma \in S_{n}} b_{\sigma} \mathbf{x}^{\sigma}}
$$

with

$$
S_{m}=\left\{\alpha \mid 0 \leq \alpha_{i} \leq m_{i}, \quad i \in I_{k}\right\}
$$

and

$$
S_{n}=\left\{\alpha \mid 0 \leq \alpha_{i} \leq n_{i}, \quad i \in I_{k}\right\}
$$

There are $\Pi_{i \in I_{k}}\left(m_{i}+1\right)+\Pi_{i \in I_{k}}\left(n_{i}+1\right)$ unknown coefficients in the equation. We note that the coefficients $a_{\mu}$ and $b_{\sigma}$ will be determined at most up to a common multiplicative factor. So, we assume that $b_{0, \ldots, 0}=\cdots=b_{0}=1$. By multiplying the difference between $f(\mathbf{x})$ and $[m / n]_{f}(\mathbf{x})$ by the denominator of $[m / n]_{f}(\mathbf{x})$, we obtain

$$
\sum_{\sigma \in S_{n}} b_{\sigma} \mathbf{x}^{\sigma} \sum_{\alpha \in S} c_{\alpha} \mathbf{x}^{\alpha}-\sum_{\mu \in S_{m}} a_{\mu} \mathbf{x}^{\mu}=\sum_{\beta \in S} d_{\beta} \mathbf{x}^{\beta}
$$

with

$$
\begin{gathered}
d_{\beta}=0 \quad \beta \in S_{m} \cup S_{n} \\
d_{\beta}=0 \quad \beta \in S_{3} \\
\sum_{\beta \in R_{4 ; p}} d_{\beta}=0 \quad p \in P
\end{gathered}
$$

with

$$
\begin{gathered}
R_{4 ; p}=\bigcup_{i \in I_{p}}\left\{\alpha \mid \alpha=m_{i}^{\prime}+n_{i}^{\prime}-p+1 ; \alpha_{j}=p_{j}, j \neq i\right\} \\
P=\left\{p \mid p \in S_{m} \cap S_{n} ; I_{p}=\left\{j \mid p_{j}=\max \left(p_{i}\right) \quad \text { with } i \in I_{k}\right\} \quad \text { has } \text { at least } 2 \text { elements }\right\}
\end{gathered}
$$

where $m_{i}^{\prime}=\min \left(m_{i}, n_{i}\right)$ and $n_{i}^{\prime}=\max \left(m_{i}, n_{i}\right)$. Next, the equations obtained by matching coefficients in equation (71) are

$$
\begin{gathered}
\sum_{\sigma \in S_{n}} b_{\sigma} c_{\mu-\sigma}=a_{\mu} \quad \mu \in S_{m} \\
\sum_{\sigma \in S_{n}} b_{\sigma} c_{\mu-\sigma}=0 \quad \mu \in\left(S_{n} \backslash S_{m}\right) \cup S_{3} \\
\sum_{\mu \in R_{4 ; p}} \sum_{\mu \in S_{n}} b_{\sigma} c_{\mu-\sigma}=0 \quad p \in P
\end{gathered}
$$

with

$$
S_{3}=\bigcup_{p \in P}\left\{\cup_{i \in I_{p}}\left\{\alpha \mid n_{i}^{\prime}<\alpha_{i} \leq n_{i}^{\prime}+m_{i}^{\prime}-p ; \alpha_{j}=p_{j}, j \neq i\right\}\right\}
$$

where $c_{\alpha}=0$ if $\alpha_{i}<0$ for at least one $i \in I_{k}$. Then, after normalising $b_{0, \ldots, 0}, \cdots, b_{0}$ to unity, the computation of the coefficients $b_{\sigma}$ can be achieved by solving the linear equations which arise from (78) and (79). Next, the linear equations given by (77) enable the coefficients $a_{\mu}$ to be determined, with the coefficients $b_{\sigma}$ found previously. 


\section{First example}

In this section a simple example with two degrees-of-freedom is presented in order to explain all the computational developments for each non-linear method and to discuss the main numerical results. In this example, we first illustrate the reduction of the order of the non-linear system at near-equilibrium point upon bifurcation, the simplification of the non-linear terms due to the fractional approximants and the approximation of the non-linear solution as a Fourier series by using a harmonic balance method. The advantages of each non-linear method will be demonstrated by comparing the non-linear behaviour obtained by using these non-linear approaches with those obtained by a classical integration scheme (Runge-Kutta of order 4). More particularly, the advantages of the fractional rational approximants after the centre manifold will be illustrated and discussed: we will demonstrate that the rational approximants have a greater range of validity than the polynomial one and that they allow us to obtain an approximation of the solution even if the associated approximation obtained by the centre manifold reduction is not sufficient or diverge.

\subsection{Non-linear model: general presentation}

In the field of mechanical engineering, the non-linear dynamical system defined in Figure (4) is a classic example of friction-induced vibrations in a brake system. It presents the grabbing vibration in heavy trucks that results from coupling between the normal mode $\left(k_{1}, m_{1}\right)$ of the brake control and the torsion mode of the front axle $\left(k_{2}, m_{2}\right)$. In order to simulate a braking system placed crosswise due to overhanging caused by a static force effect, we consider the moving belt slopes with an angle $\theta$. This slope couples the normal and tangential degree-of-freedom induced only by the friction coefficient $\mu$ that is assumed to be constant. The braking force $F_{\text {brake }}$ transits through the braking command, that has non-linear behaviour. Therefore, we consider the possibility of a non-linear contribution. This non-linearity is applied in order to indicate the influence and the importance of non-linear terms in under-standing the dynamic behaviour of systems with non-linear phenomena, the prediction of dangerous or favourable conditions, and the exploitation of the full capability of structures by using systems in the non-linear range. In this study, the nonlinear behaviour dynamic of the brake command of the system $\left(k_{1}, m_{1}\right)$, and the non-linear behaviour dynamic of the front axle assembly and the suspension $\left(k_{2}, m_{2}\right)$ are concerned, respectively. These non-linearities are defined as non-linear stiffnesses. The non-linear behaviour is then expressed as a quadratic and cubic polynomial in the relative displacement:

$$
\begin{aligned}
& k_{1}=k_{11}+k_{12}(Y-y)+k_{13}(Y-y)^{2} \\
& k_{2}=k_{21}+k_{22} X+k_{23} X^{2}
\end{aligned}
$$

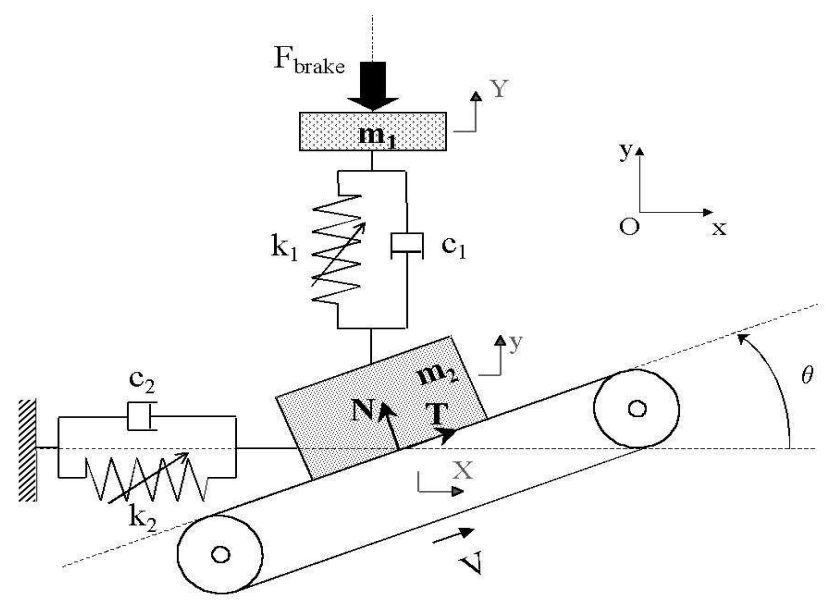

Figure 4: Non-linear model of the braking system

With reference to Figure 4, and considering the non-linear expression of the stiffnesses defined in equations (81), 
the three equations of motion can be expressed as

$$
\left\{\begin{array}{l}
m_{1} \ddot{Y}+c_{1}(\dot{Y}-\dot{y})+k_{11}(Y-y)+k_{12}(Y-y)^{2}+k_{13}(Y-y)^{3}=-F_{\text {brake }} \\
m_{2} \ddot{X}+c_{2} \dot{X}+k_{21} X+k_{22} X^{2}+k_{23} X^{3}=-N \sin \theta+T \cos \theta \\
m_{2} \ddot{y}+c_{1}(\dot{y}-\dot{Y})+k_{11}(y-Y)+k_{12}(y-Y)^{2}+k_{13}(y-Y)^{3}=N \cos \theta+T \sin \theta
\end{array}\right.
$$

Considering Coulomb's friction law $T=\mu N$, and the transformations $y=X \tan \theta$ and $\mathbf{x}=\{X \quad Y\}^{T}$, the nonlinear 2-degrees-of-freedom system is given by

$$
\mathbf{M} \ddot{\mathbf{x}}+\mathbf{C} \dot{\mathbf{x}}+\mathbf{K x}=\mathbf{F}+\mathbf{F}_{\mathrm{NL}}(\mathbf{x})
$$

where $\ddot{\mathrm{x}}, \ddot{\mathrm{x}}$ and $\mathrm{x}$ define the acceleration, velocity, and displacement response 2-dimensional vectors of the degreesof-freedom, respectively. $\mathbf{M}, \mathbf{C}$ and $\mathbf{K}$ are the mass, damping and stiffness matrices of the mechanical system. $\mathbf{F}$ defines the vector due to the brake force, and $\mathbf{F}_{\mathbf{N L}}(\mathbf{x})$ is the vector containing all the non-linear terms of the system (82). By considering the equations of (82) the expressions of all the matrices and vectors are

$$
\begin{gathered}
\mathbf{M}=\left[\begin{array}{cc}
m_{2}\left(\tan ^{2} \theta+1\right) & 0 \\
0 & m_{1}
\end{array}\right] \\
\mathbf{C}=\left[\begin{array}{cc}
c_{1}\left(\tan ^{2} \theta-\mu \tan \theta\right)+c_{2}(1+\mu \tan \theta) & c_{1}(-\tan \theta+\mu) \\
-c_{1} \tan \theta & c_{1}
\end{array}\right] \\
\mathbf{K}=\left[\begin{array}{c}
k_{21}(1+\mu \tan \theta)+k_{11}\left(\tan ^{2} \theta-\mu \tan \theta\right) \\
k_{11}(-\tan \theta+\mu) \\
-k_{11} \tan \theta
\end{array}\right] \\
\mathbf{F}_{\mathbf{N L}}=\left\{\begin{array}{c}
(-\tan \theta+\mu)\left(k_{12}(X \tan \theta-Y)^{2}+k_{13}(X \tan \theta-Y)^{3}\right) \\
+k 22(1+\mu \tan \theta) X^{2}+k_{23}(1+\mu \tan \theta) X^{3} \\
-k_{12}(Y-X \tan \theta)^{2}-k_{13}(Y-X \tan \theta)^{3}
\end{array}\right\} \\
\mathbf{F}=\left\{\begin{array}{c}
0 \\
-F_{\text {brake }}
\end{array}\right\}
\end{gathered}
$$

The general form of the equation of motion for the non-linear system is given in the following way:

$$
\mathbf{M} \ddot{\mathbf{x}}+\mathbf{C} \dot{\mathbf{x}}+\mathbf{K} \mathbf{x}=\mathbf{F}+\sum_{i=1}^{2} \sum_{j=1}^{2} \mathbf{f}_{(\mathbf{2})}^{\mathbf{i j}} x_{i} x_{j}+\sum_{i=1}^{2} \sum_{j=1}^{2} \sum_{k=1}^{2} \mathbf{f}_{(\mathbf{3})}^{\mathbf{i j k}} x_{i} x_{j} x_{k}
$$

where $\mathbf{f}_{(\mathbf{2})}^{\mathrm{ij}}$ et $\mathbf{f}_{(\mathbf{3})}^{\mathrm{ijk}}$ are the vectors of quadratic and cubic non-linear terms, respectively.

\subsection{Stability analysis}

\subsubsection{Methodology}

The stability analysis is the first step in instability phenomena and allows us to obtain the stable and unstable areas versus the evolution of parameters. This methodology can be divided into two parts; firstly, the equilibrium point of the non-linear system is obtained by solving the non-linear static equations for a given parameter. Next, stability analysis is investigated by the determination of eigenvalues of the linearized equations for each steady-state operating point of the non-linear system or by calculating the Jacobian of the system. The linearized equations are obtained by introducing small perturbations about the equilibrium point into the non-linear equations. The equilibrium point $\mathbf{x}_{\mathbf{0}}$ of the non-linear system (89) satisfies the following conditions:

$$
\mathbf{K}_{\mathbf{0}}=\mathbf{F}+\mathbf{F}_{\mathbf{N L}}\left(\mathbf{x}_{\mathbf{0}}\right)
$$


Then, stability of the non-linear system is investigated on the linearized equations by assuming small perturbations $\overline{\mathbf{x}}=\left\{\begin{array}{ll}\bar{X} & \bar{Y}\end{array}\right\}^{T}$ about the equilibrium point $\mathbf{x}_{\mathbf{0}}=\left\{\begin{array}{ll}X_{0} & Y_{0}\end{array}\right\}^{T}$ of the non-linear system :

$$
\mathbf{x}=\mathbf{x}_{\mathbf{0}}+\overline{\mathbf{x}}
$$

By substituting equations (91) in the linearised expression of the non-linear system (89), we obtain the linearized system

$$
\mathbf{M} \ddot{\overline{\mathbf{x}}}+\mathbf{C} \dot{\overline{\mathbf{x}}}+\mathbf{K}\left(\overline{\mathbf{x}}+\mathbf{x}_{\mathbf{0}}\right)=\mathbf{F}+\mathbf{F}_{\mathbf{N L}}\left(\mathbf{x}_{\mathbf{0}}\right)+\mathbf{F}_{\mathbf{L}}(\overline{\mathbf{x}})
$$

with

$$
\mathbf{F}_{\mathbf{L}}(\overline{\mathbf{x}})=\left[\begin{array}{cc}
\left.\frac{\partial F_{N L}^{X}}{\partial \bar{X}}\right|_{\mathbf{x}_{\mathbf{0}}} & \left.\frac{\partial F_{N L}^{X}}{\partial \bar{Y}}\right|_{\mathbf{x}_{\mathbf{0}}} \\
\left.\frac{\partial F_{N L}^{Y}}{\partial \bar{X}}\right|_{\mathbf{x}_{\mathbf{0}}} & \left.\frac{\partial F_{N L}^{Y}}{\partial \bar{Y}}\right|_{\mathbf{x}_{\mathbf{0}}}
\end{array}\right] . \overline{\mathbf{x}}
$$

The terms of the vector $\mathbf{F}_{\mathbf{L}}(\overline{\mathbf{x}})=\left\{F_{L}^{X}(\overline{\mathbf{x}}) \quad F_{L}^{Y}(\overline{\mathbf{x}})\right\}^{T}$ at the equilibrium point $\mathbf{x}_{\mathbf{0}}$ for small perturbations $\overline{\mathbf{x}}$ are given by

$$
\begin{aligned}
& F_{L}^{X}(\overline{\mathbf{x}})=(-\tan \theta+\mu)\left(2 k_{12}\left(\tan ^{2} \theta X_{0} \bar{X}+Y_{0} \bar{Y}-\tan \theta Y_{0} \bar{X}-\tan \theta X_{0} \bar{Y}\right)\right. \\
&\left.+3 k_{13}\left(\tan ^{3} \theta X_{0}^{2} \bar{X}+2 \tan ^{2} \theta X_{0} Y_{0} \bar{X}+\tan \theta Y_{0}^{2} \bar{X}+2 \tan \theta X_{0} Y_{0} \bar{Y}-Y_{0}^{2} \bar{Y}\right)\right) \\
&+(1+\mu \tan \theta)\left(2 k_{22} X_{0} \bar{X}+3 k_{23} X_{0}^{2} \bar{X}-\tan ^{2} \theta X_{0}^{2} \bar{Y}\right) \\
& F_{L}^{Y}(\overline{\mathbf{x}})=-2 k_{12}\left(Y_{0} \bar{Y}+\tan \theta Y_{0} \bar{X}+\tan ^{2} \theta X_{0} \bar{X}-\tan \theta X_{0} \bar{Y}-\tan \theta Y_{0} \bar{X}\right) \\
&+3 k_{13}\left(2 \tan \theta X_{0} Y_{0} \bar{X}-Y_{0}^{2} \bar{Y}+\tan \theta Y_{0}^{2} \bar{X}-\tan ^{2} \theta X_{0}^{2} \bar{Y}-2 \tan ^{2} \theta X_{0} Y_{0} \bar{X}+\tan ^{3} \theta X_{0}^{2} \bar{X}\right)
\end{aligned}
$$

Finally, the linearized equation of the non-linear system at the equilibrium point $\mathbf{x}_{\mathbf{0}}$ is given by

$$
\mathbf{M} \ddot{\overline{\mathbf{x}}}+\mathbf{C} \dot{\overline{\mathbf{x}}}+\left(\mathbf{K}-\mathbf{K}_{\mathbf{L}}\right) \overline{\mathbf{x}}=\mathbf{0}
$$

with

$$
\mathbf{F}_{\mathbf{L}}(\overline{\mathbf{x}})=\mathbf{K}_{\mathbf{L}} \overline{\mathbf{x}}
$$

Then, the stability analysis may be carried out by determining the eigenvalues of the matrix $\mathbf{A}$ given by

$$
\mathbf{A}=\left[\begin{array}{cc}
\mathbf{0} & \mathbf{I} \\
-\mathbf{M}^{-1}\left(\mathbf{K}-\mathbf{K}_{\mathbf{L}}\right) & -\mathbf{M}^{-1} \mathbf{C}
\end{array}\right]
$$

The eigenvalues $\lambda$ of $A$ can be expressed as

$$
\lambda=a+i b
$$

where $a$ is the real part, and $b$ is the imaginary part of the eigenvalue, respectively. If all eigenvalues have their real part $a$ negative or zero, the system is stable. If one or more eigenvalues have their real part $a$ positive, the system is unstable. Therefore, $b$ represents frequency of the unstable mode. It clearly appears that the stability analysis may be applied for large non-linear systems and practical computational implementation can be easy and systematic.

Another classic approach for investigating the stability of non-linear systems is the Routh-Hurwitz criteria [48]. In this case, the fourth-degree characteristic polynomial of the linearized system (96) is investigated. It has the form $\lambda^{4}+a_{3} \lambda^{3}+a_{2} \lambda^{2}+a_{1} \lambda+a_{0}=0$. The system is stable if the three following relations are verified: (a) $a_{3}>0$; (b) $a_{2} a_{3}-a_{1}>0$; (c) $a_{1}\left(a_{2} a_{3}-a_{1}\right)-a_{1} a_{3}^{2}>0$. Even if this approach has advantages in determining analytical expressions of stability criteria versus all the parameters of the non-linear system, the estimate of these expressions is very difficult to obtain for non-linear systems with many degrees-of-freedom. 


\subsubsection{Hopf bifurcation point}

Moreover, one of the important points is the determination of the Hopf bifurcation point. This point defines the limit between the stable and unstable areas of non-linear systems. Moreover, the non-linear behaviour of an unstable dynamical system is usually estimated around this Hopf bifurcation point when the system becomes unstable. The Hopf bifurcation point can be defined as follows

$$
\left\{\begin{array}{l}
\left.\operatorname{Re}\left(\lambda_{\text {center }}(\mu)\right)\right|_{\mathbf{x}=\mathbf{x}_{\mathbf{0}}, \mu=\mu_{0}}=0 \\
\left.\operatorname{Re}\left(\lambda_{\text {no-center }}(\mu)\right)\right|_{\mathbf{x}_{\mathbf{x}=\mathbf{x}_{\mathbf{0}}, \mu=\mu_{0}} \neq 0} \neq 0 \\
\left.\frac{d}{d \mu}(\operatorname{Re}(\lambda(\mu)))\right|_{\mu=\mu_{0}} \neq 0
\end{array}\right.
$$

The first condition implies that the system (96) has a pair of purely imaginary eigenvalues $\lambda_{\text {centre }}$ while all of its other eigenvalues $\lambda_{n o-c e n t r e}$ have nonzero real parts at $\left(\mathbf{x}=\mathbf{x}_{\mathbf{0}}, \mu=\mu_{0}\right)$. The last condition of (113), called a transversally condition, implies a transversal or nonzero speed crossing of the imaginary axis, as shown in Figure 6.

\subsubsection{Computational stability analysis}

The following parameters will be used for the computational stability analysis and the associated parametric studies: the friction coefficient $\mu=0.3$; the brake force $F_{\text {brake }}=1 N$; the equivalent mass of the first and second modes $m_{1}=m_{2}=1 \mathrm{~kg}$; the equivalent damping of the first and second modes $c_{1}=c_{2}=5 \mathrm{~N} / \mathrm{m} / \mathrm{sec}$; the coefficients of the linear, quadratic and cubic terms of the stiffness $k_{1}$ for the first mode, $k_{11}=1.10^{5} \mathrm{~N} / \mathrm{m}, k_{12}=1.10^{6} \mathrm{~N} / \mathrm{m}$ and $k_{13}=1.10^{6} \mathrm{~N} / \mathrm{m}$, respectively; the coefficients of the linear, quadratic and cubic terms of the stiffness $k_{2}$ for the second mode, $k_{12}=1.10^{6} \mathrm{~N} / \mathrm{m}, k_{12}=1.10^{5} \mathrm{~N} / \mathrm{m}$ and $k_{13}=1.10^{5} \mathrm{~N} / \mathrm{m}$, respectively; the angle $\theta=0.2 \mathrm{rad}$.

Firstly, the computations are conducted with respect to the brake friction coefficient $\mu$. The Hopf bifurcation point is detected for $\mu=\mu_{0}=0.204$. A representation of the evolution of frequencies against brake friction coefficient $\mu$ is given in Figure 5. In Figure 6, the associated real parts are plotted; the real part of eigenvalues is negative when $\mu<\mu_{0}$. As the friction coefficient $\mu$ increases, the two modes move closer until they reach the bifurcation zone. We obtain the coalescence for $\mu=\mu_{0}$ of two imaginary parts of the eigenvalues (frequency about $50 \mathrm{~Hz}$ ). For $\mu=\mu_{0}$, there is one pair of purely imaginary eigenvalues. All other eigenvalues have negative real parts. After the bifurcation, the real part of eigenvalues is positive. In conclusion, the system is unstable for $\mu \leq \mu_{0}$, and stable for $\mu>\mu_{0}$.

Then, stability analysis versus two parameters can be easily carried out by numerically determining the evolution of the real part and imaginary part of the eigenvalues. For example, parametric studies are shown in Figures 7-14; Figures 7(a)-14(a) indicate the stable and unstable areas versus the evolution of parameters and Figures 7(b)-14(b) show the evolution of the frequencies in the complex plane. Consequently, stability analysis is a very complex problem: stable and unstable regions can be obtained by varying parameters and there are an infinite number of combinations of parameters that could be examined. This is why computational parametric studies are very interesting and useful in order to obtain general indications for parametric design studies. 


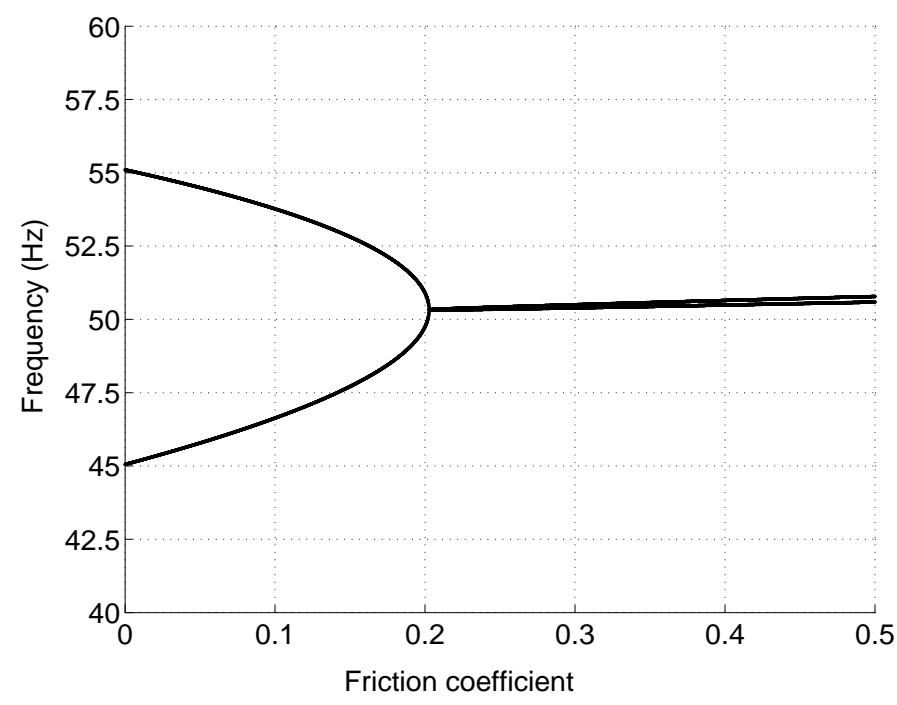

Figure 5: Evolution of the frequencies versus the friction coefficient

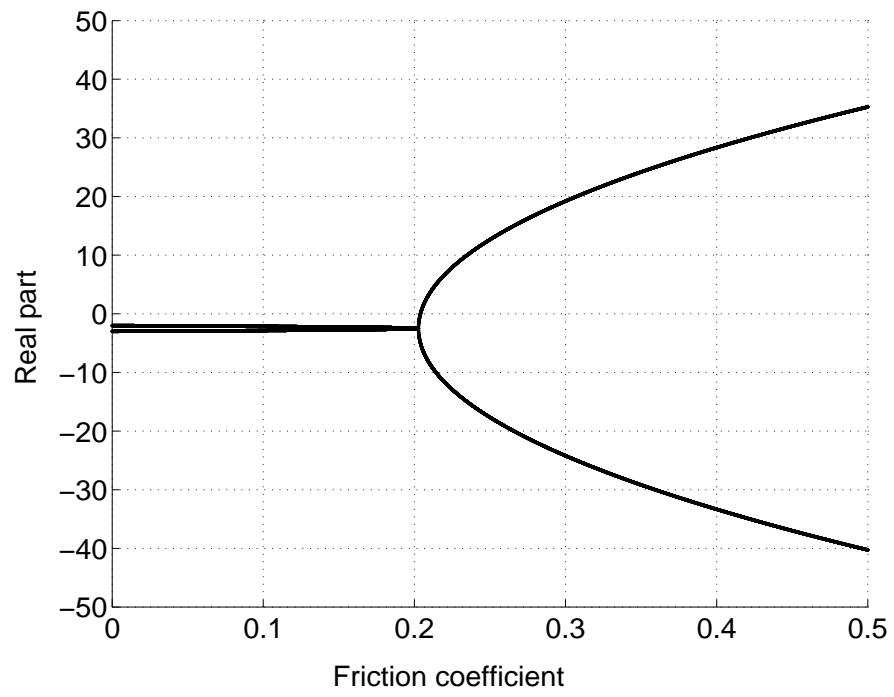

Figure 6: Evolution of the real parts versus the friction coefficient

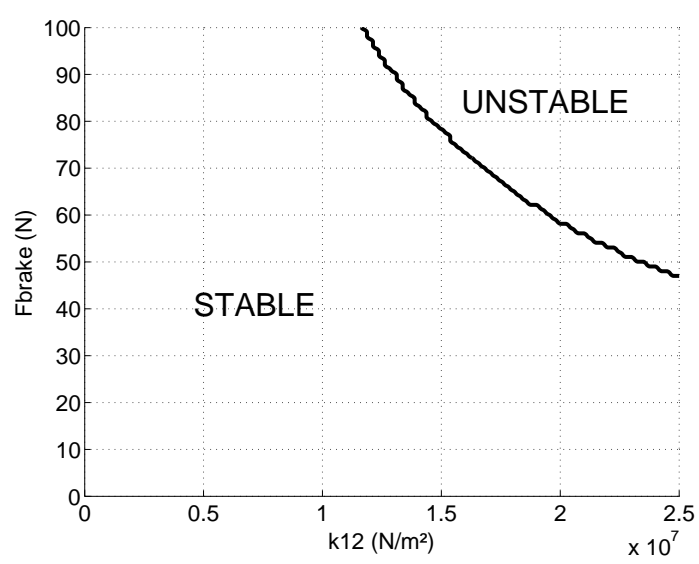

(a) Stable/Unstable areas

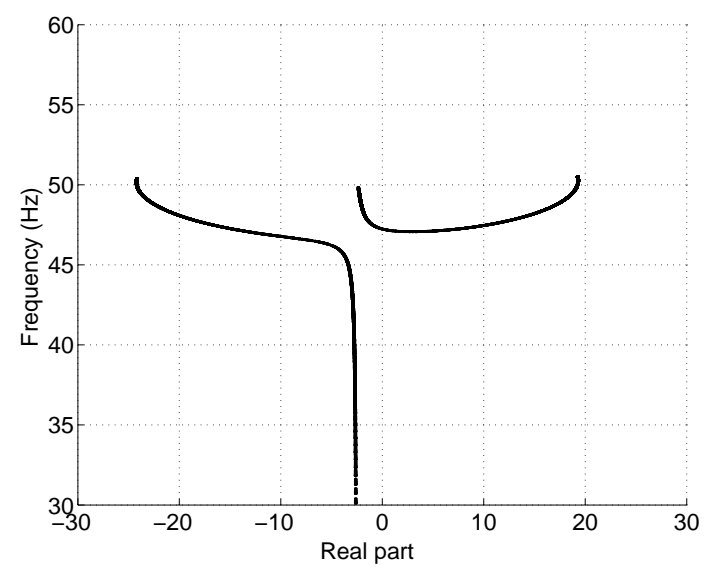

(b) Evolution of frequencies

Figure 7: Stability analysis versus $F_{\text {brake }}$ and $k_{12}$ 


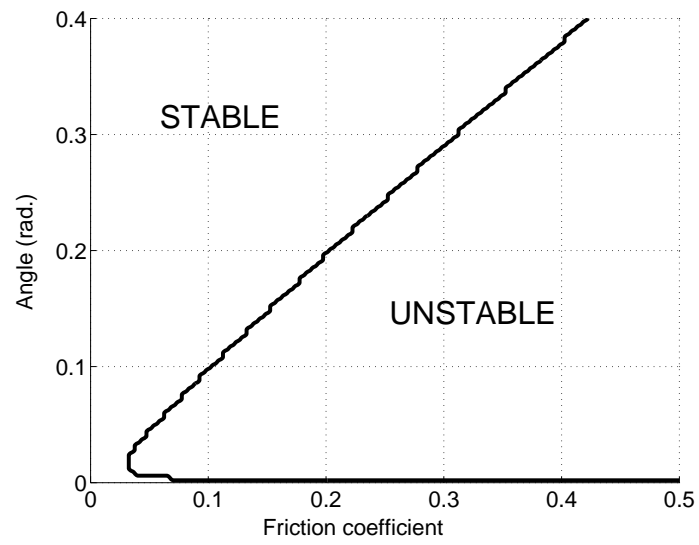

(a) Stable/Unstable areas

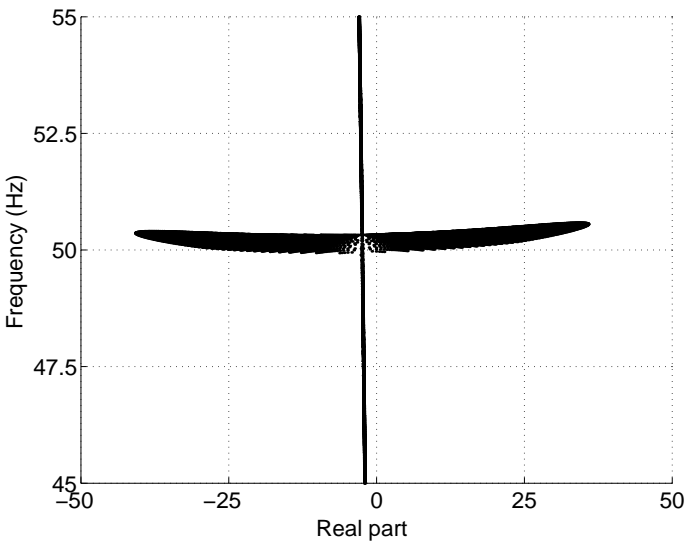

(b) Evolution of frequencies

Figure 8: Stability analysis versus the sprag-slip angle and the friction coefficient

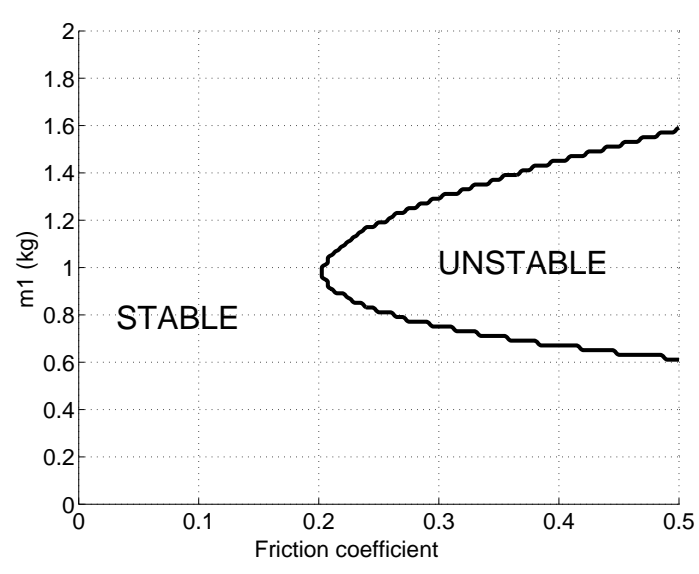

(a) Stable/Unstable areas

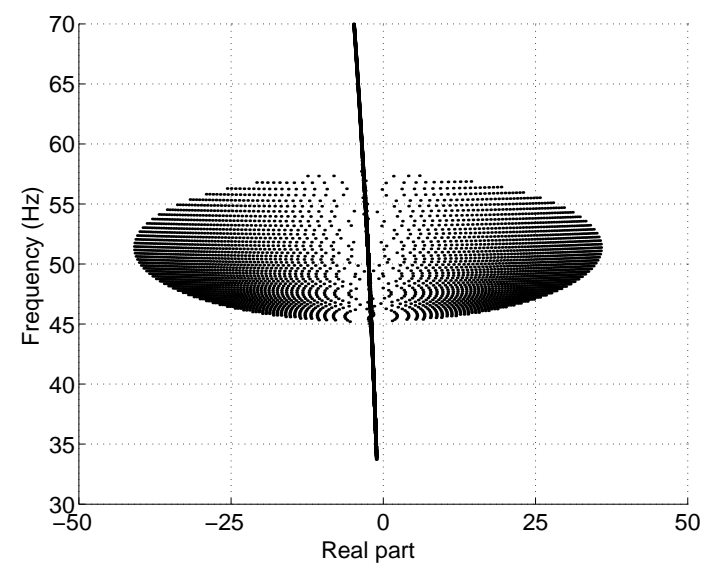

(b) Evolution of frequencies

Figure 9: Stability analysis versus the friction coefficient and $m_{1}$

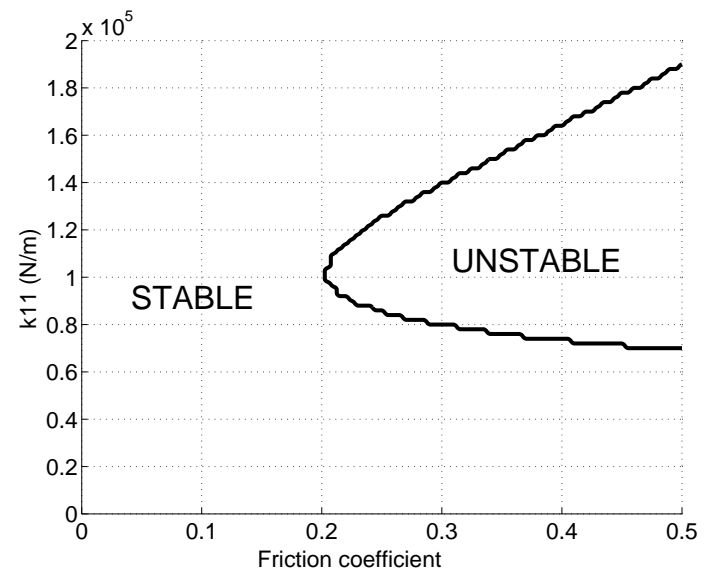

(a) Stable/Unstable areas

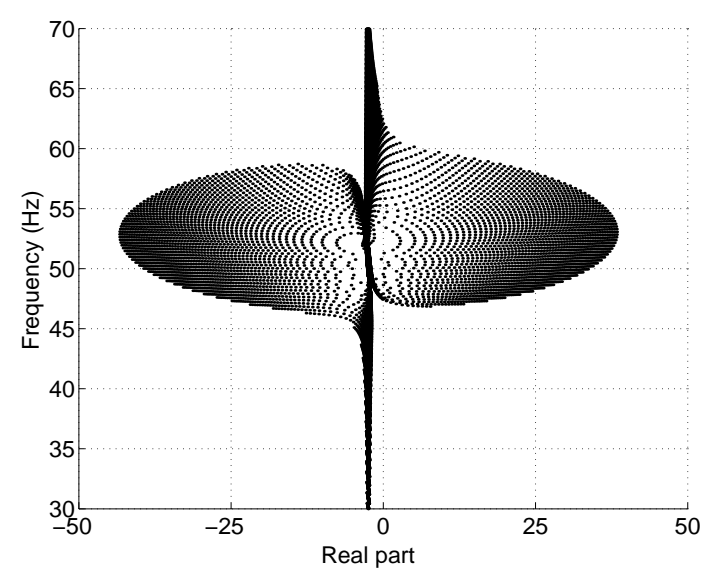

(b) Evolution of frequencies

Figure 10: Stability analysis versus the the friction coefficient and $k_{11}$ 


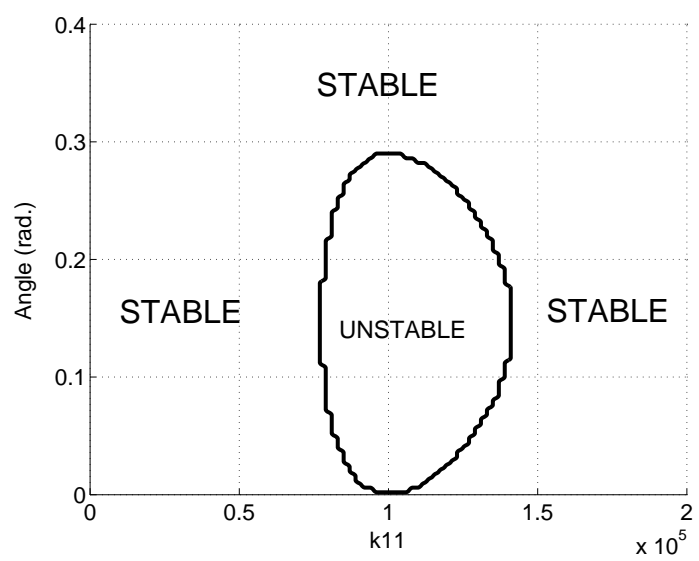

(a) Stable/Unstable areas

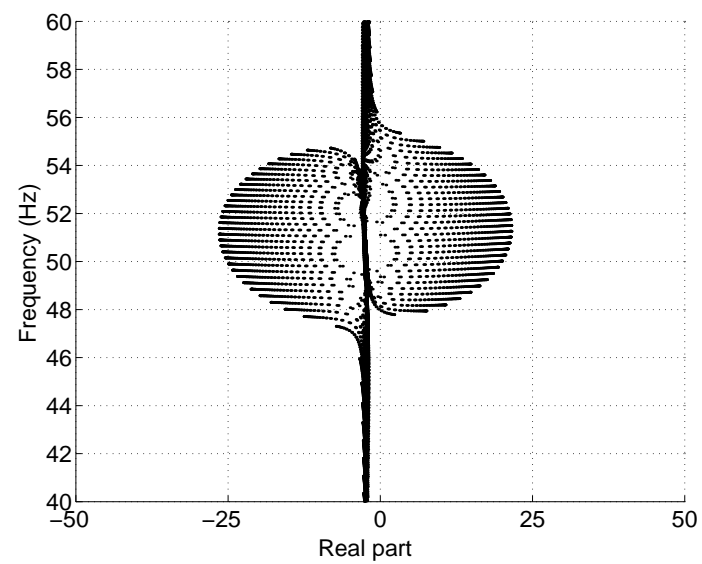

(b) Evolution of frequencies

Figure 11: Stability analysis versus the sprag-slip angle and $k_{11}$

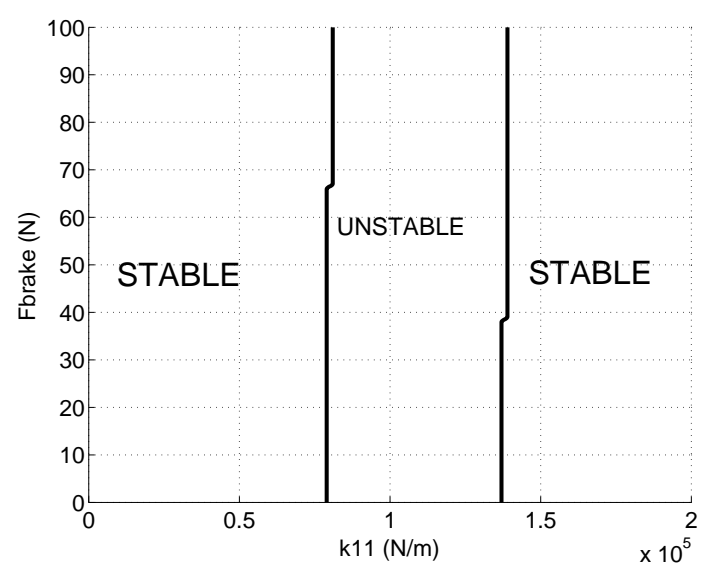

(a) Stable/Unstable areas

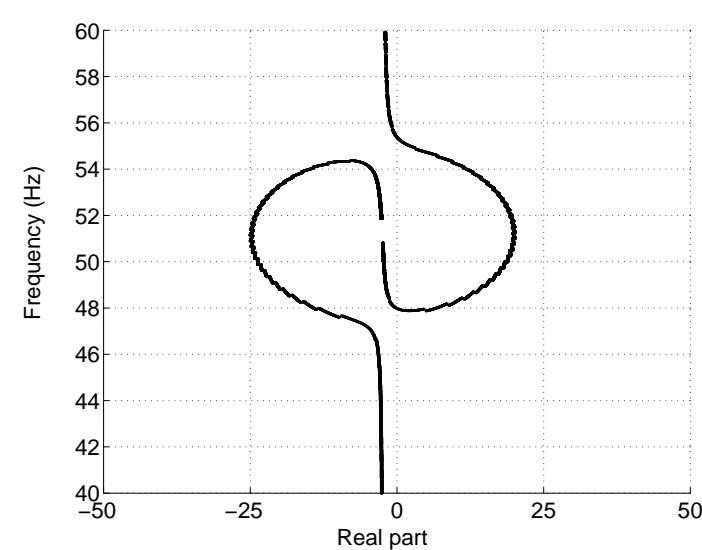

(b) Evolution of frequencies

Figure 12: Stability analysis versus $k_{11}$ and $F_{\text {brake }}$

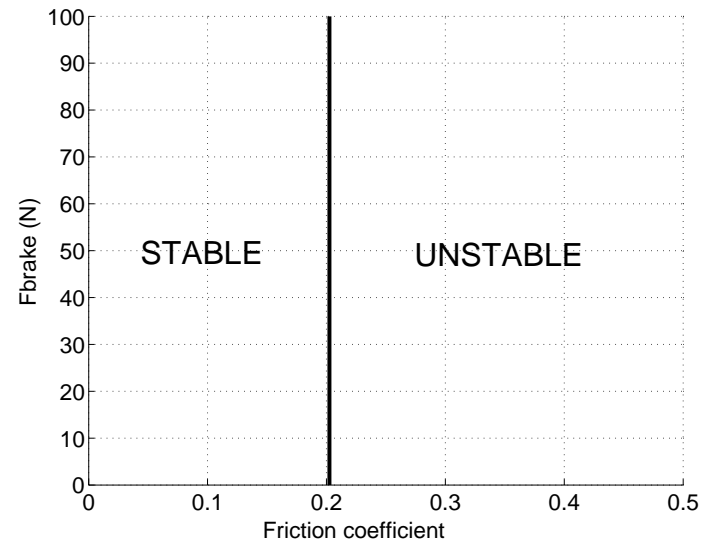

(a) Stable/Unstable areas

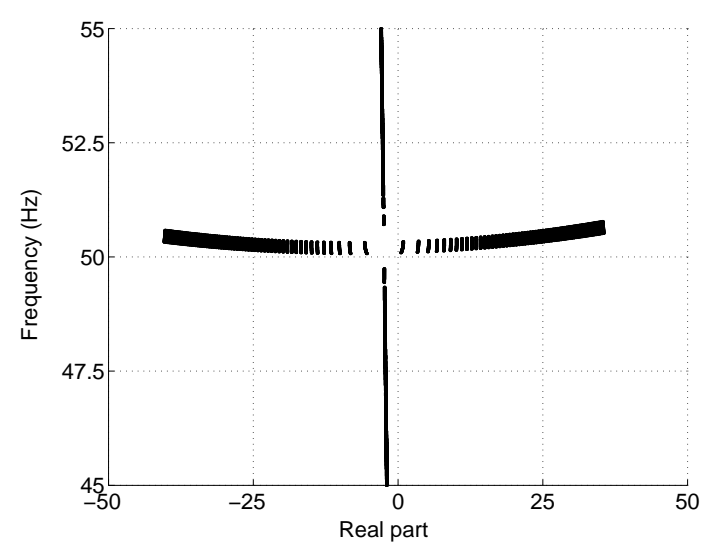

(b) Evolution of frequencies

Figure 13: Stability analysis versus the friction coefficient and $F_{b r a k e}$ 


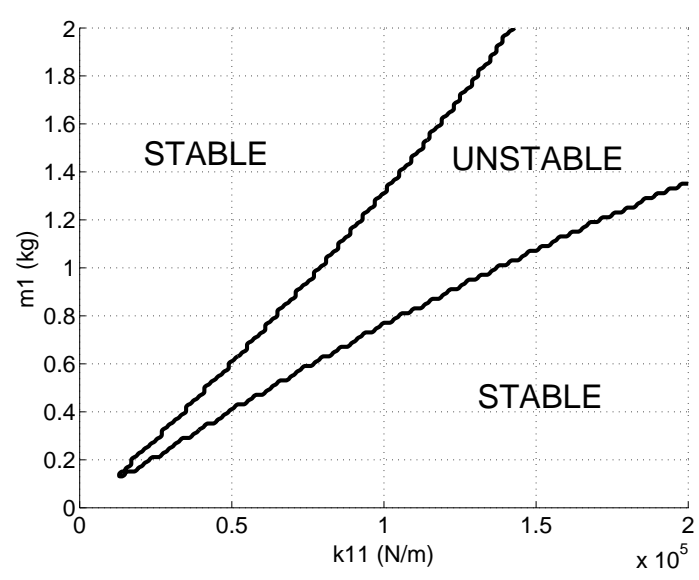

(a) Stable/Unstable areas

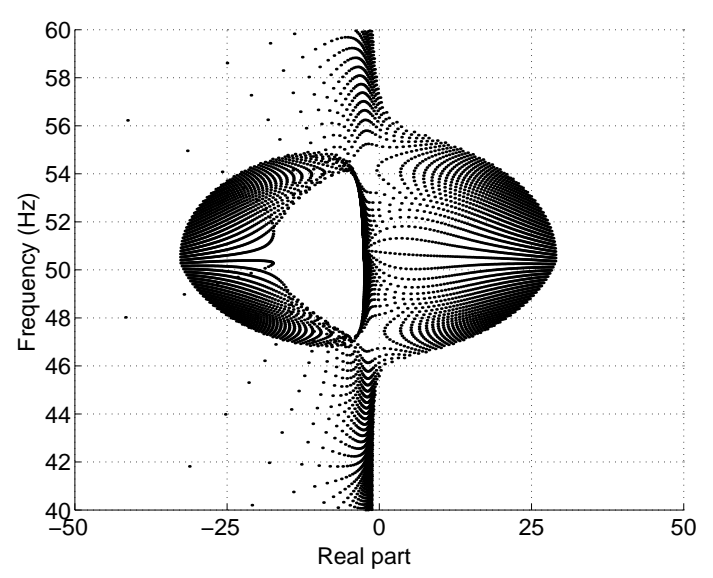

(b) Evolution of frequencies

Figure 14: Stability analysis versus $k_{11}$ and $m_{1}$

\subsection{Non-linear dynamic: classical approach}

While stability analysis conducted using the determination of the eigenvalues of the linearized equation at the equilibrium point are extremely useful in evaluating the effect of changes in various parameters, they can not evaluate the vibration amplitudes at the instability region and more particularly near the Hopf bifurcation point. In this case, the time-history solutions of the full set of the non-linear equations (89) can evaluate the non-linear dynamic behaviour of a system in near-critical steady-state equilibrium point.

These time-history responses of the non-linear system can be calculated by using a classic fourth-order Runge-Kutta algorithm. Figures 15 and Figures 16 show the transient response analysis and the predicted non-linear vibration amplitudes of the displacement $X(t)$ and the velocity $\dot{X}(t)$ at the instability region $\mu=1.001 \mu_{0}$, respectively. We observe that the displacement $X(t)$ and velocity $\dot{X}(t)$ grow until we obtain the periodic oscillations of the non-linear dynamical behaviour of the system. Then, the evolution of the associated limit cycle amplitude $(X, \dot{X})$ can be evaluated at the instability region $\mu=1.001 \mu_{0}$, as illustrated in Figure 17. The time-history of the displacement $Y(t)$ and the velocity $\dot{Y}(t)$ at the instability region $\mu=1.001 \mu_{0}$ are plotted in Figure 18 and 19, respectively; the associated limit cycle amplitude $(Y, \dot{Y})$ is shown in Figure 20.

Even if the time-history response solutions have been obtained using a fourth-order Runge-Kutta algorithm to integrate the non-linear equations (89), this procedure is rather expensive and consumes considerable resources both in terms of the computation time and in terms of the data storage requirements when extensive parametric design studies are needed. As explained in Section 2.1, various non-linear methods can be applied to find the non-linear response of this dynamical system. For example, it may be possible to assume the non-linear vibration amplitudes of the displacements $X(t)$ and $Y(t)$ as truncated Fourier series. The numerical estimate of this solution should be obtained by applying such non-linear methods as the trigonometric collocation method, and the various harmonic balance methods for example ([132], [134], [149], [111], [109], [143], [113], [25], [129] and [174]). All these numerical methods can be commonly applied to solve non-linear problems; however, the most efficient methods are those that first reduce and simplify the non-linear original system. Then we may use the non-linear methods that enable approximation of the solution. By adopting this step and a succession of non-linear methods, each method is used in an optimal way and the final reduced and approximated system can be considered as one of the simplest forms possible. So an understanding of the behaviour of the non-linear system first requires simplification and reduction of the equations. In order to obtain the non-linear reduced and simplified system, the centre manifold approach and the rational fractional approximants will be used in this study. 


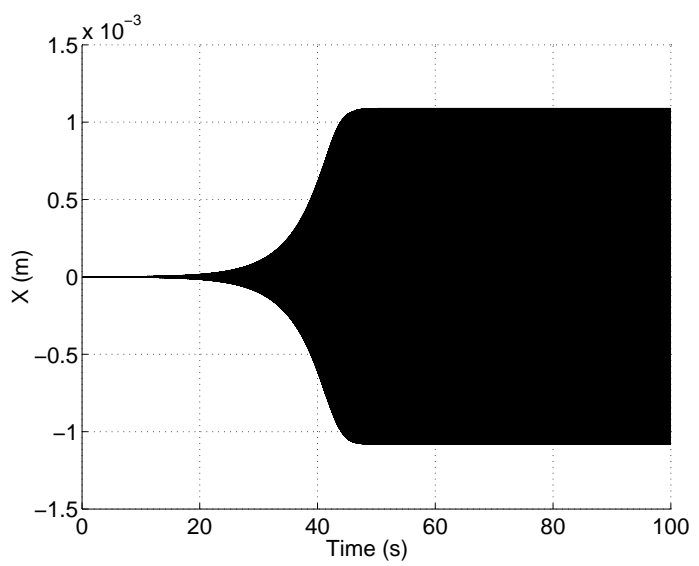

(a) Evolution of the displacement

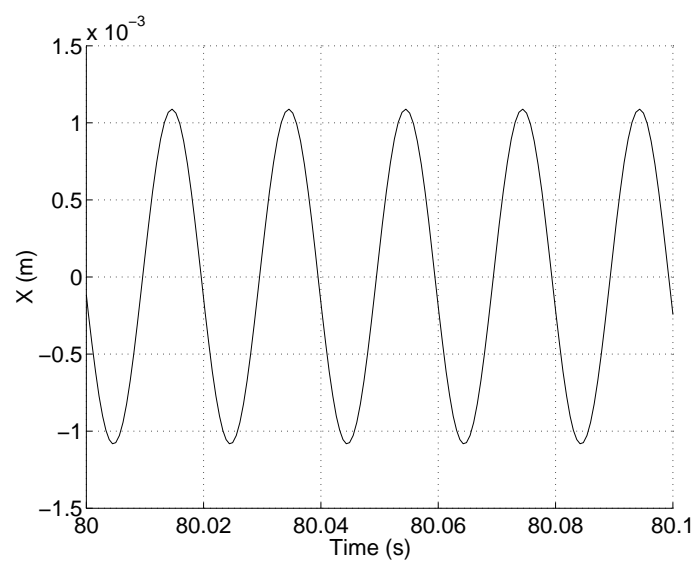

(b) Zoom

Figure 15: Evolution of the displacement $X(t)$ for $\mu=1.001 \mu_{0}$ by using Runge-Kutta 4

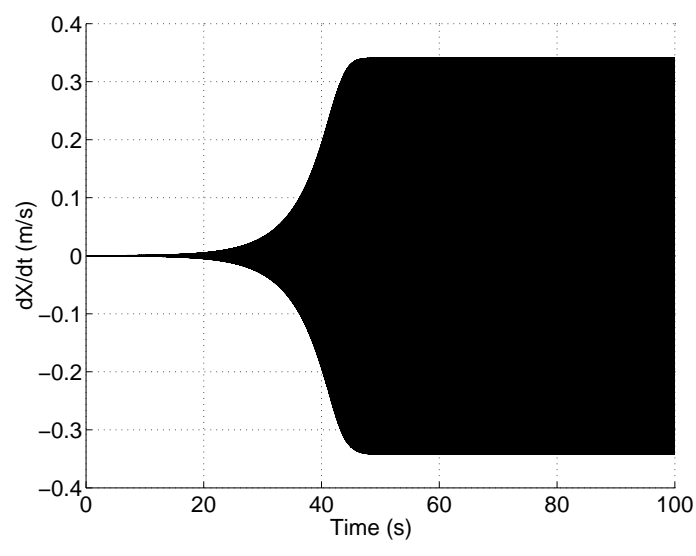

(a) Evolution of the velocity

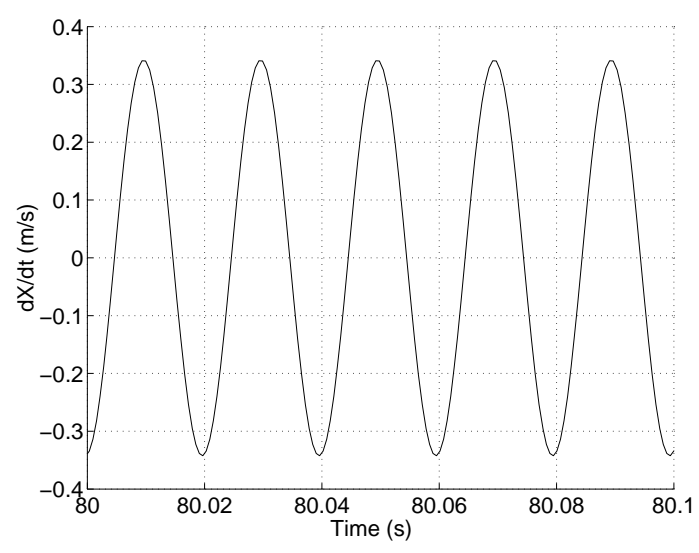

(b) Zoom

Figure 16: Evolution of the velocity $\dot{X}(t)$ for $\mu=1.001 \mu_{0}$ by using Runge-Kutta 4

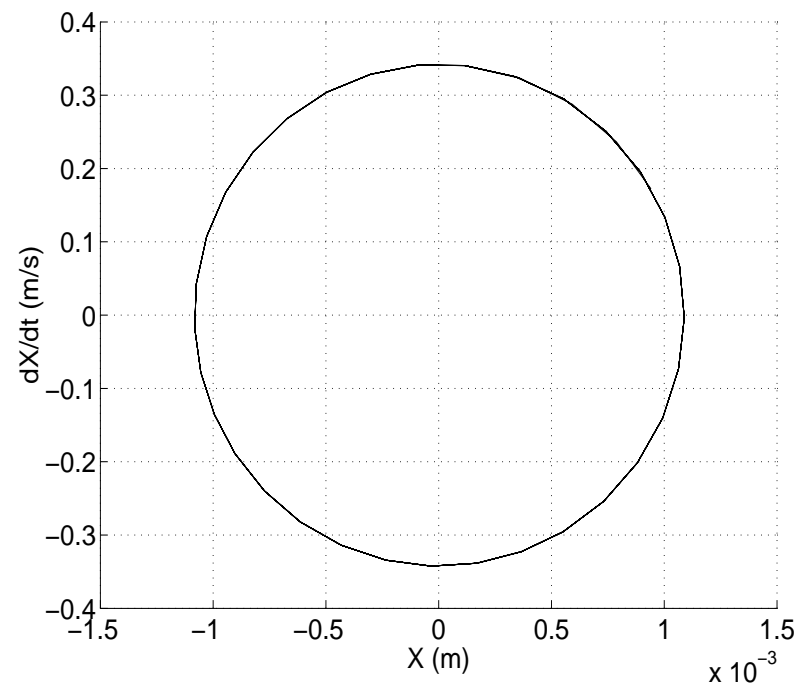

Figure 17: Limit cycles $(X, \dot{X})$ for $\mu=1.001 \mu_{0}$ by using Runge-Kutta 4 


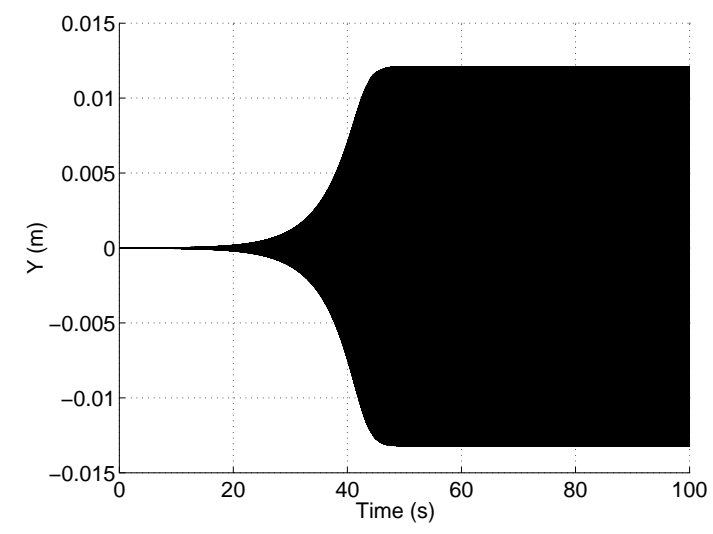

(a) Evolution of the displacement

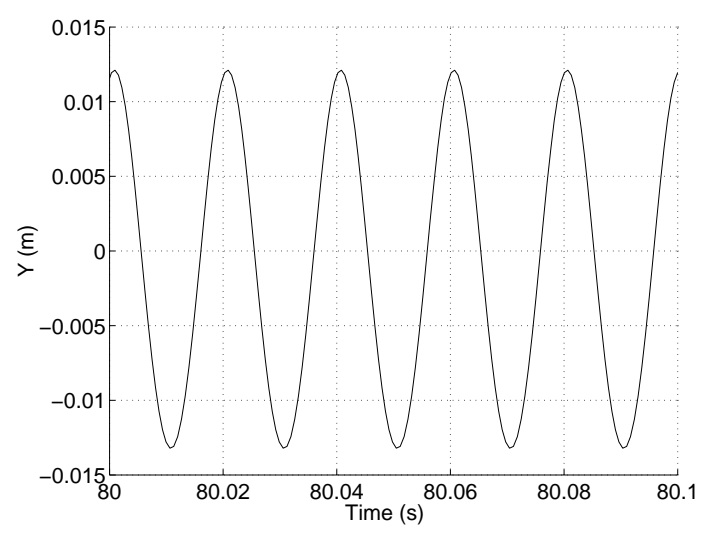

(b) Zoom

Figure 18: Evolution of the displacement $Y(t)$ for $\mu=1.001 \mu_{0}$ by using Runge-Kutta 4

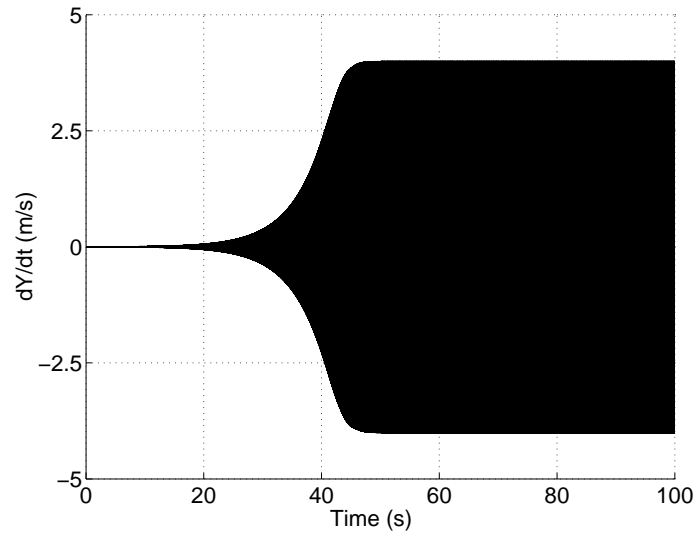

(a) Evolution of the velocity

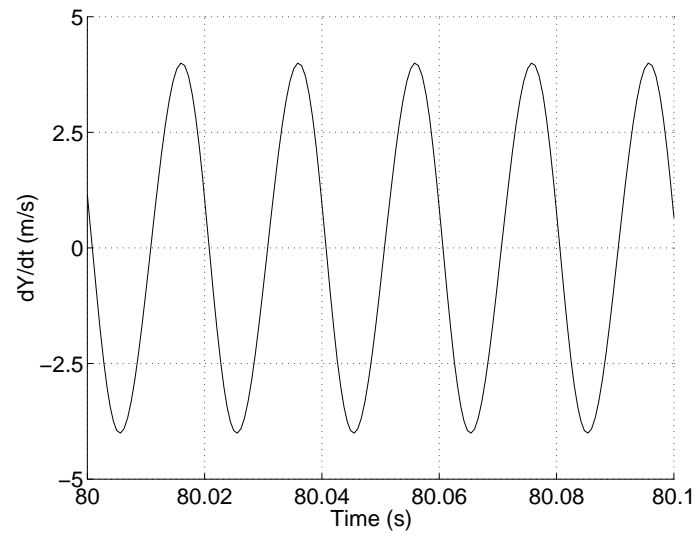

(b) Zoom

Figure 19: Evolution of the velocity $\dot{Y}(t)$ for $\mu=1.001 \mu_{0}$ by using Runge-Kutta 4

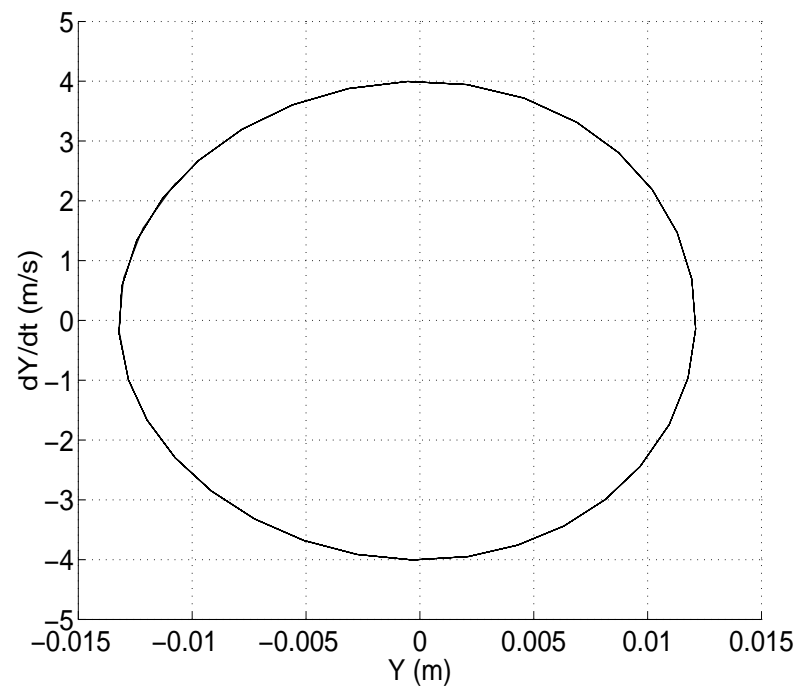

Figure 20: Limit cycles $(Y, \dot{Y})$ for $\mu=1.001 \mu_{0}$ by using Runge-Kutta 4 


\subsection{Reduced non-linear system via the centre manifold approach}

\subsubsection{Non-linear formulation of the problem}

In order to apply the centre manifold approach to reduce and to simplify the original non-linear system without losing the effect of the non-linear terms, the complete non-linear sytem (89) may be rewritten about the equilibrium point $\mathbf{x}_{\mathbf{0}}$ for small perturbations $\overline{\mathbf{x}}$ in state variables $\mathbf{y}=\left\{\begin{array}{ll}\mathbf{x} & \dot{\mathbf{x}}\end{array}\right\}^{T}$.

The complete non-linear equation of the system (89) is given by

$$
\mathbf{M} \ddot{\overline{\mathbf{x}}}+\mathbf{C} \dot{\overline{\mathbf{x}}}+\mathbf{K} \overline{\mathbf{x}}=\mathbf{P}_{\mathbf{N L}}(\overline{\mathbf{x}})
$$

where $\ddot{\overline{\mathbf{x}}}, \dot{\overline{\mathrm{x}}}$ et $\overline{\mathrm{X}}$ are the acceleration, velocity and displacement response two-dimensional vectors of the degrees of freedom, respectively. $\mathbf{M}, \mathbf{C}$ and $\mathbf{K}$ are the mass matrix, the damping matrix and the stiffness matrix, respectively. $\mathbf{P}_{\mathrm{NL}}=\left\{P_{N L}^{X}(\overline{\mathbf{x}}) \quad P_{N L}^{Y}(\overline{\mathbf{x}})\right\}^{T}$ contains the linear and non-linear terms of the system. By considering equations (93-95), $\mathbf{P}_{\mathrm{NL}}$ has the form

$$
\begin{aligned}
& P_{N L}^{X}(\overline{\mathbf{x}})=F_{L}^{X}(\overline{\mathbf{x}})+F_{N L}^{X}(\overline{\mathbf{x}}) \\
& P_{N L}^{Y}(\overline{\mathbf{x}})=F_{L}^{Y}(\overline{\mathbf{x}})+F_{N L}^{Y}(\overline{\mathbf{x}})
\end{aligned}
$$

where $F_{L}^{X}(\overline{\mathbf{x}})$ et $F_{L}^{Y}(\overline{\mathbf{x}})$ are the linear terms of $P_{N L}^{X}(\overline{\mathbf{x}})$ and $P_{N L}^{Y}(\overline{\mathbf{x}})$ at the equilibrium point $\mathbf{x}_{\mathbf{0}}$, as defined in equations (93) and (95). $F_{N L}^{X}(\overline{\mathbf{x}})$ and $F_{N L}^{Y}(\overline{\mathbf{x}})$ defines the purely non-linear term of $P_{N L}^{X}(\overline{\mathbf{x}})$ et $P_{N L}^{Y}(\overline{\mathbf{x}})$ at the equilibrium point $\mathbf{x}_{\mathbf{0}}$. These expressions are given by

$$
\begin{aligned}
F_{N L}^{X}= & k_{12}(-\tan \theta+\mu)\left(\tan ^{2} \theta \bar{X}^{2}+\bar{Y}^{2}-2 \tan \theta \overline{Y X}\right)+k_{13}(-\tan \theta+\mu)\left(\tan ^{3} \theta\left(\bar{X}^{3}+3 \bar{X}^{2} X_{0}\right)\right. \\
& \left.-3 \tan ^{2} \theta\left(\overline{Y X}^{2}+2 \overline{Y X} X_{0}+\bar{X}^{2} X_{0}\right)+3 \tan \theta\left(\overline{X Y}^{2}+\overline{Y X} Y_{0}+\bar{Y}^{2} X Y_{0}\right)-\bar{Y}^{3}-3 \bar{Y}^{2} Y_{0}\right) \\
& +k_{22}(1+\mu \tan \theta) \bar{X}^{2}+k_{23}(1+\mu \tan \theta)\left(\bar{X}^{3}+3 \bar{X}^{2} X_{0}\right) \\
F_{N L}^{Y}= & k_{12}\left(\bar{Y}^{2}-2 \tan \theta \overline{X Y}+\tan ^{2} \theta \bar{X}^{2}\right)+k_{13}\left(\bar{Y}^{3}+3 \bar{Y}^{2} Y_{0}\right)-3 \tan \theta\left(\bar{Y}^{2} \bar{X}+2 \overline{X Y} Y_{0}+\bar{Y}^{2} X_{0}\right) \\
& +3 \tan ^{2} \theta\left(\bar{X}^{2} \bar{Y}+2 \overline{Y Y} X_{0}+\bar{X}^{2} Y_{0}\right)-\tan ^{3}\left(\bar{X}^{3}+3 \bar{X}^{2} Y_{0}\right)
\end{aligned}
$$

Then, the non-linear system can be rewritten at the equilibrium point $\mathbf{x}_{\mathbf{0}}=\left\{\begin{array}{ll}X_{0} & Y_{0}\end{array}\right\}^{T}$ for small perturbations $\overline{\mathbf{x}}=\left\{\begin{array}{ll}\bar{X} & \bar{Y}\end{array}\right\}^{T}$

$$
\mathbf{M} \ddot{\overline{\mathbf{x}}}+\mathbf{C} \dot{\overline{\mathbf{x}}}+\mathbf{K} \overline{\mathbf{x}}=\sum_{i=1}^{2} \mathbf{f}_{(\mathbf{1})}^{\mathbf{i}} \overline{x_{i}}+\sum_{i=1}^{2} \sum_{j=1}^{2} \mathbf{f}_{(\mathbf{2})}^{\mathbf{i} \mathbf{j}} \overline{x_{i} x_{j}}+\sum_{i=1}^{2} \sum_{j=1}^{2} \sum_{k=1}^{2} \mathbf{f}_{(\mathbf{3})}^{\mathbf{i j k}} \overline{x_{i} x_{j} x_{k}}
$$

where the coefficients $\mathbf{f}_{(\mathbf{1})}^{\mathrm{i}}, \mathbf{f}_{(\mathbf{2})}^{\mathrm{ij}}$ and $\mathbf{f}_{(\mathbf{3})}^{\mathrm{ijk}}$ define the linear, quadratic and cubic terms of the system.

By rearranging the linear terms on the left with $\tilde{\mathbf{K}} \overline{\mathbf{x}}=\mathbf{K} \overline{\mathbf{x}}-\sum_{i=1}^{2} \mathbf{f}_{(\mathbf{1})}^{\mathbf{i}} \overline{x_{i}}$ the expression of the non-linear system is

$$
\mathbf{M} \ddot{\overline{\mathbf{x}}}+\mathbf{C} \dot{\overline{\mathbf{x}}}+\tilde{\mathbf{K}} \overline{\mathbf{x}}=\sum_{i=1}^{2} \sum_{j=1}^{2} \mathbf{f}_{(\mathbf{2})}^{\mathbf{i j}} \overline{x_{i} x_{j}}+\sum_{i=1}^{2} \sum_{j=1}^{2} \sum_{k=1}^{2} \mathbf{f}_{(\mathbf{3})}^{\mathrm{ijk}} \overline{x_{i} x_{j} x_{k}}
$$

Finally, the non-linear equations of the system are written in state variables $\mathbf{y}=\left\{\begin{array}{ll}\overline{\mathbf{x}} & \dot{\overline{\mathbf{x}}}\end{array}\right\}^{T}$

$$
\dot{\mathbf{y}}=\mathbf{A y}+\sum_{i=1}^{4} \sum_{j=1}^{4} \mathbf{q}_{(\mathbf{2})}^{\mathbf{i j}} y_{i} y_{j}+\sum_{i=1}^{4} \sum_{j=1}^{4} \sum_{k=1}^{4} \mathbf{q}_{(\mathbf{3})}^{\mathbf{i j} \mathbf{k}} y_{i} y_{j} y_{k}
$$

with

$$
\mathbf{A}=\left[\begin{array}{cc}
\mathbf{C} & \mathbf{M} \\
\mathbf{I} & \mathbf{0}
\end{array}\right]^{-1}\left[\begin{array}{cc}
\tilde{\mathbf{K}} & \mathbf{0} \\
\mathbf{0} & \mathbf{I}
\end{array}\right]
$$




$$
\begin{aligned}
& \mathbf{q}_{(2)}=\left[\begin{array}{cc}
\mathbf{C} & \mathbf{M} \\
\mathbf{I} & \mathbf{0}
\end{array}\right]^{-1}\left\{\begin{array}{c}
\mathbf{f}_{(2)} \\
\mathbf{0}
\end{array}\right\} \\
& \mathbf{q}_{(3)}=\left[\begin{array}{cc}
\mathbf{C} & \mathbf{M} \\
\mathbf{I} & 0
\end{array}\right]^{-1}\left\{\begin{array}{c}
\mathbf{f}_{(3)} \\
\mathbf{0}
\end{array}\right\}
\end{aligned}
$$

where $\mathbf{q}_{(\mathbf{2})}^{\mathrm{ij}}$ and $\mathbf{q}_{(\mathbf{3})}^{\mathrm{ijk}}$ are the quadratic and cubic non-linear terms of the system (107) in state variables $\mathbf{y}=\{\overline{\mathbf{x}} \quad \dot{\overline{\mathbf{x}}}\}^{T}$.

\subsubsection{Application of the centre manifold approach}

The centre manifold method allows reduction of a non-linear system to a lower-dimensional form near the Hopf bifurcation point. As explained in Section 2.2, the previous system (108) can be written at the Hopf bifurcation point in the form

$$
\left\{\begin{array}{l}
\dot{\mathbf{v}_{\mathbf{c}}}=\mathbf{J}_{\mathbf{c}} \mathbf{v}_{\mathbf{c}}+\mathbf{G}_{\mathbf{2}}\left(\mathbf{v}_{\mathbf{c}}, \mathbf{v}_{\mathbf{s}}\right)+\mathbf{G}_{\mathbf{3}}\left(\mathbf{v}_{\mathbf{c}}, \mathbf{v}_{\mathbf{s}}\right) \\
\dot{\mathbf{v}_{\mathbf{s}}}=\mathbf{J}_{\mathbf{s}} \mathbf{v}_{\mathbf{s}}+\mathbf{H}_{\mathbf{2}}\left(\mathbf{v}_{\mathbf{c}}, \mathbf{v}_{\mathbf{s}}\right)+\mathbf{H}_{\mathbf{3}}\left(\mathbf{v}_{\mathbf{c}}, \mathbf{v}_{\mathbf{s}}\right)
\end{array}\right.
$$

In this example, we consider the physically interesting case of the stable equilibrium losing stability and the first coupling modes that is the most commonly studied cases in the field of mechanical engineering. So, there are two centre variables $\mathbf{v}_{\mathbf{c}}=\left\{\begin{array}{ll}v_{c 1} & v_{c 2}\end{array}\right\}^{T}$ and two stable variables $\mathbf{v}_{\mathbf{s}}=\left\{\begin{array}{ll}v_{s 1} & v_{s 2}\end{array}\right\}^{T} . \mathbf{J}_{\mathbf{c}}$ and $\mathbf{J}_{\mathbf{s}}$ have eigenvalues $\lambda$ such that $\operatorname{Re}\left(\lambda_{\mathbf{J}_{\mathbf{c}}}\left(\mu_{0}\right)\right)=0$ and $\operatorname{Re}\left(\lambda_{\mathbf{J}_{\mathbf{s}}}\left(\mu_{0}\right)\right) \neq 0 . \mathbf{G}_{\mathbf{2}}, \mathbf{G}_{\mathbf{3}}, \mathbf{H}_{\mathbf{2}}$ and $\mathbf{H}_{\mathbf{3}}$ are the matrices containing the quadratic and cubic terms for the centre variables $\mathbf{v}_{\mathbf{c}}$ and the stable variables $\mathbf{v}_{\mathbf{s}}$, respectively. All the properties of $\mathbf{G}_{\mathbf{2}}, \mathbf{G}_{\mathbf{3}}, \mathbf{H}_{\mathbf{2}}$ and $\mathbf{H}_{3}$ are given in Section 2.2.

In this study, we consider the application of the centre manifold approach near the Hopf bifucation point. In this case, the previous system (112) is augmented with the control/unfolding parameter $\hat{\mu}=\mu_{0}+\bar{\mu}$ as follows

$$
\left\{\begin{array}{l}
\dot{\mathbf{v}_{\mathbf{c}}}=\mathbf{J}_{\mathbf{c}}(\hat{\mu}) \mathbf{v}_{\mathbf{c}}+\mathbf{G}_{\mathbf{2}}\left(\mathbf{v}_{\mathbf{c}}, \mathbf{v}_{\mathbf{s}}, \hat{\mu}\right)+\mathbf{G}_{\mathbf{3}}\left(\mathbf{v}_{\mathbf{c}}, \mathbf{v}_{\mathbf{s}}, \hat{\mu}\right) \\
\dot{\mathbf{v}}_{\mathbf{s}}=\mathbf{J}_{\mathbf{s}}(\hat{\mu}) \mathbf{v}_{\mathbf{s}}+\mathbf{H}_{\mathbf{2}}\left(\mathbf{v}_{\mathbf{c}}, \mathbf{v}_{\mathbf{s}}, \hat{\mu}\right)+\mathbf{H}_{\mathbf{3}}\left(\mathbf{v}_{\mathbf{c}}, \mathbf{v}_{\mathbf{s}}, \hat{\mu}\right) \\
\dot{\hat{\mu}}=0
\end{array}\right.
$$

At the point $\left(\mathbf{v}_{\mathbf{c}}, \mathbf{v}_{\mathbf{s}}, \hat{\mu}\right)=(\mathbf{0}, \mathbf{0}, 0)$, the non-linear system has three centre variables with the centre space $\left(\mathbf{v}_{\mathbf{c}}, \hat{\mu}\right)$ associated. As explained in Section 2.2 the centre manifold theory (Carr [28]) allows the expression of the stable variables $\mathbf{v}_{\mathbf{s}}$ as a power series $\mathbf{h}$ in $\left(\mathbf{v}_{\mathbf{c}}, \hat{\mu}\right)$ of degree $p$ (with $p<1$ ). We obtain

$$
\mathbf{v}_{\mathbf{s}}=\mathbf{h}\left(\mathbf{v}_{\mathbf{c}}, \hat{\mu}\right)=\sum_{p=i+j+l=2}^{m} \sum_{j=0}^{p} \sum_{l=0}^{p} \mathbf{a}_{\mathbf{i j} \mathbf{1}} v_{c 1}^{i} v_{c 2}^{j} \hat{\mu}^{l}
$$

where $\mathbf{a}_{\mathbf{i j l}}$ is the vector of the centre manifold coefficients. We recall that the polynomial approximations $\mathbf{h}$ do not contain constant and linear terms in order to verify the tangency conditions at the Hopf bifurcation point to the centre eigenspace: $\mathbf{h}(\mathbf{0}, \mathbf{0}, 0)=\mathbf{0} ; D_{\mathbf{v}_{\mathbf{c}}} h_{i}(\mathbf{0}, \mathbf{0}, 0)=\mathbf{0}$ for $1 \leq i \leq 2 ; \frac{\partial \mathbf{h}}{\partial \hat{\mu}}(\mathbf{0}, \mathbf{0}, 0)=0$.

More precisely, the stable variables are defined by

$$
\mathbf{v}_{\mathbf{s}}=\left\{\begin{array}{l}
v_{s 1} \\
v_{s 2}
\end{array}\right\}=\left\{\begin{array}{l}
h_{1}\left(\mathbf{v}_{\mathbf{c}}\right) \\
h_{2}\left(\mathbf{v}_{\mathbf{c}}\right)
\end{array}\right\}=\left\{\begin{array}{c}
\sum_{p=i+j+l=2}^{m} \sum_{j=0}^{p} \sum_{l=0}^{p} a_{1, i j l} v_{c 1}^{i} v_{c 2}^{j} \hat{\mu}^{l} \\
\sum_{p=i+j+l=2}^{m} \sum_{j=0}^{p} \sum_{l=0}^{p} a_{2, i j l} v_{c 1}^{i} v_{c 2}^{j} \hat{\mu}^{l}
\end{array}\right\}
$$

It may be noted that the terms $v_{c 1} \hat{\mu}, v_{c 2} \hat{\mu}, v_{s 1} \hat{\mu}$ et $v_{s 2} \hat{\mu}$ are now used as non-linear quadratic terms. The value of the coefficients $a_{k, i j l}$ (with $1 \leq k \leq 2$ ) is obtained by solving the following system

$$
\begin{array}{r}
D_{\mathbf{v}_{\mathbf{c}}, \hat{\mu}}\left(\mathbf{h}\left(\mathbf{v}_{\mathbf{c}}, \hat{\mu}\right)\right)\left(\mathbf{J}_{\mathbf{c}} \mathbf{v}_{\mathbf{c}}+\mathbf{G}_{\mathbf{2}}\left(\mathbf{v}_{\mathbf{c}}, \mathbf{h}\left(\mathbf{v}_{\mathbf{c}}, \hat{\mu}\right), \hat{\mu}\right)+\mathbf{G}_{\mathbf{3}}\left(\mathbf{v}_{\mathbf{c}}, \mathbf{h}\left(\mathbf{v}_{\mathbf{c}}, \hat{\mu}\right), \hat{\mu}\right)\right) \\
=\mathbf{J}_{\mathbf{s}} \mathbf{h}\left(\mathbf{v}_{\mathbf{c}}, \hat{\mu}\right)+\mathbf{H}_{\mathbf{2}}\left(\mathbf{v}_{\mathbf{c}}, \mathbf{h}\left(\mathbf{v}_{\mathbf{c}}, \hat{\mu}\right), \hat{\mu}\right)+\mathbf{H}_{\mathbf{3}}\left(\mathbf{v}_{\mathbf{c}}, \mathbf{h}\left(\mathbf{v}_{\mathbf{c}}, \hat{\mu}\right), \hat{\mu}\right)
\end{array}
$$

By substituting the assumed polynomial approximation $\mathbf{h}$ into (116), and equating the coefficients of the different terms in the polynomials on both side, a system of algebraic equations is obtained and the coefficients $a_{k, i j l}$ (with 
$1 \leq k \leq 2)$ are obtained.

Then, the reduced system is defined by

$$
\left\{\begin{array}{l}
\dot{\mathbf{v}}_{\mathbf{c}}=\mathbf{J}_{\mathbf{c}}(\hat{\mu}) \mathbf{v}_{\mathbf{c}}+\mathbf{G}_{\mathbf{2}}\left(\mathbf{v}_{\mathbf{c}}, \mathbf{h}\left(\mathbf{v}_{\mathbf{c}}, \hat{\mu}\right), \hat{\mu}\right)+\mathbf{G}_{\mathbf{3}}\left(\mathbf{v}_{\mathbf{c}}, \mathbf{h}\left(\mathbf{v}_{\mathbf{c}}, \hat{\mu}\right), \hat{\mu}\right) \\
\dot{\hat{\mu}}=0
\end{array}\right.
$$

Even though the centre manifold approach is very interesting due to the reduction of the system to a lower-dimensional form near the bifurcation point, we observe that determining the coefficients $a_{k, i j l}$ (with $1 \leq k \leq n$ where $n$ defining the number of the stable variables is a complex problem. It is easy to obtain the analytical expressions of these coefficients for a small-dimensional system with few non-linear terms. However, in the case of large-dimensional systems with complex non-linear terms, it would be impossible to obtain an analytical expression of the coefficient $a_{k, i j l}$ (with $1 \leq k \leq 2$ ) due to the complexity of the polynomial approximations and the important number of non-linearities where the centre, stable and unstable variables are non-linearly coupled.

In the next section, we propose a computational procedure to determine the numerical values of the coefficients $a_{k, i j l}$ (with $1 \leq k \leq n$ where $n$ is the number of stable variables) in the general case of a $p$-dimensional system with three centre variables $\left(v_{c 1}, v_{c 2}, \hat{\mu}\right)$ and $n$ stable variables $\mathbf{v}_{\mathbf{s}}=\left\{\begin{array}{llll}v_{s 1} & v_{s 2} & \cdots & v_{s n}\end{array}\right\}^{T}$. The general form of the non-linear system (113) is

$$
\left\{\begin{array}{l}
\dot{\mathbf{v}}_{\mathbf{c}}=\mathbf{J}_{\mathbf{c}} \mathbf{v}_{\mathbf{c}}+\left[G_{(2)}^{i j}\right] \mathbf{v} \otimes \mathbf{v}+\left[G_{(3)}^{i k}\right] \mathbf{v} \otimes \mathbf{v} \otimes \mathbf{v} \\
\dot{\mathbf{v}}_{\mathbf{s}}=\mathbf{J}_{\mathbf{s}} \mathbf{v}_{\mathbf{s}}+\left[H_{(2)}^{i j}\right] \mathbf{v} \otimes \mathbf{v}+\left[H_{(3)}^{i k}\right] \mathbf{v} \otimes \mathbf{v} \otimes \mathbf{v} \\
\dot{\hat{\mu}}=0
\end{array}\right.
$$

with $\mathbf{v}=\left\{\begin{array}{lll}\mathbf{v}_{\mathbf{c}}{ }^{T} & \mathbf{v}_{\mathbf{s}}^{T} & \hat{\mu}\end{array}\right\}^{T} . \otimes$ defines the Kronecker product [180]. We assume that $\operatorname{dim}\left(\mathbf{v}_{\mathbf{c}}\right)=2$ and $\operatorname{dim}\left(\mathbf{v}_{\mathbf{s}}\right)=$ $n$. So, that $\operatorname{dim}(\mathbf{v})=n+3 . G_{(2)}^{i j}, G_{(3)}^{i k}, H_{(2)}^{l j}$ and $H_{(3)}^{l k}$ are the quadratic and cubic terms (with $1 \leq i \leq 2,1 \leq l \leq n$, $1 \leq j \leq(n+3)^{2}$ and $\left.1 \leq k \leq(n+3)^{3}\right)$.

\subsubsection{Computational determination of the second order approximation}

The computational procedure applied for determining the centre manifold coefficients consists of a systematic method using the increasing power of equation (116).

If the stable variables are approximated by using only a second order polynomial expression in the centre variables $\left\{\begin{array}{ll}\mathbf{v}_{\mathbf{c}}{ }^{T} & \hat{\mu}\end{array}\right\}^{T}=\left\{\begin{array}{lll}v_{c 1} & v_{c 2} & \hat{\mu}\end{array}\right\}^{T}$, the expressions of the stable variables $\mathbf{v}_{\mathbf{s}}$ are given by

$$
\begin{aligned}
\mathbf{v}_{\mathbf{S}} & =\mathbf{h}^{(\mathbf{1})}\left(\mathbf{v}_{\mathbf{c}}, \hat{\mu}\right)=\sum_{p=i+j+l=2}^{2} \sum_{j=0}^{p} \sum_{l=0}^{p} \mathbf{a}_{\mathbf{i j 1}} v_{c 1}^{i} v_{c 2}^{j} \hat{\mu}^{l} \\
& =\mathbf{a}_{\mathbf{2 0 0}} v_{c 1}^{2}+\mathbf{a}_{\mathbf{1 1 0}} v_{c 1} v_{c 2}+\mathbf{a}_{\mathbf{0 2 0}} v_{c 2}^{2}+\mathbf{a}_{\mathbf{1 0 1}} v_{c 1} \hat{\mu}+\mathbf{a}_{\mathbf{0 1 1}} v_{c 2} \hat{\mu}+\mathbf{a}_{\mathbf{0 0 2}} \hat{\mu}^{2}
\end{aligned}
$$

with $\mathbf{a}_{200}, \mathbf{a}_{110}, \mathbf{a}_{020}, \mathbf{a}_{101}, \mathbf{a}_{011}$ and $\mathbf{a}_{002}$ the $n$-dimensional unknown vectors of the stable variables. The determination of the $n \times 6$ coefficients (with $n$ the number of the stable variables) is obtained by considering only the second order terms of (116) that are defined by

$$
D_{\mathbf{v}_{\mathbf{c}}, \hat{\mu}}\left(\mathbf{h}^{(\mathbf{1})}\left(\mathbf{v}_{\mathbf{c}}, \hat{\mu}\right)\right) \mathbf{J}_{\mathbf{c}} \mathbf{v}_{\mathbf{c}}-\mathbf{J}_{\mathbf{s}} \mathbf{h}^{(\mathbf{1})}\left(\mathbf{v}_{\mathbf{c}}, \hat{\mu}\right)-\mathbf{H}_{\mathbf{2}}\left(\mathbf{v}_{\mathbf{c}}, \hat{\mu}\right)=\mathbf{0}
$$

This expression is the exact system for second order polynomial approximation. By considering the $k^{\text {th }}$-vector of the second order coefficients for the $k^{\text {th }}$-state variable

$$
\mathbf{a}_{\mathbf{k}}^{\mathbf{2}}=\left\{\begin{array}{llllll}
a_{k, 200} & a_{k, 110} & a_{k, 020} & a_{k, 101} & a_{k, 011} & a_{k, 002}
\end{array}\right\}^{T}
$$


and by equating the coefficients of the different terms in the polynomials on both sides, the relation (120) has the form

$$
\left[\begin{array}{ccccc}
\mathbf{J}_{1,2} & 0 & \cdots & \cdots & \mathbf{0} \\
\mathbf{0} & \ddots & \ddots & & \vdots \\
\vdots & \ddots & \mathbf{J}_{\mathrm{k}, 2} & \ddots & \vdots \\
\vdots & & \ddots & \ddots & \mathbf{0} \\
\mathbf{0} & \cdots & \cdots & \mathbf{0} & \mathbf{J}_{\mathrm{n}, 2}
\end{array}\right]\left\{\begin{array}{c}
\mathbf{a}_{1}^{2} \\
\vdots \\
\mathbf{a}_{\mathrm{k}}^{2} \\
\vdots \\
\mathbf{a}_{\mathrm{n}}^{2}
\end{array}\right\}=\left\{\begin{array}{c}
\mathrm{C}_{1,2} \\
\vdots \\
\mathbf{C}_{\mathrm{k}, 2} \\
\vdots \\
\mathbf{C}_{\mathrm{n}, 2}
\end{array}\right\}
$$

where

$$
\mathbf{J}_{\mathbf{k}, \mathbf{2}}=\operatorname{diag}\left[2 J_{c 1}-J_{s k} \quad J_{c 1}+J_{c 2}-J_{s k} \quad 2 J_{c 2}-J_{s k} \quad J_{c 1}-J_{s k} \quad J_{c 2}-J_{s k} \quad J_{s k}\right]
$$

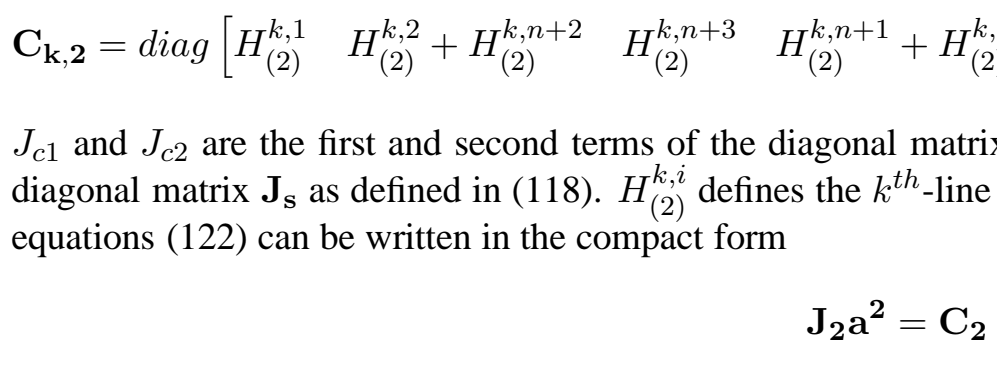

$$
\left.H_{(2)}^{k, 2(n+1)}+H_{(2)}^{k, n(n+1)+2}-H_{(2)}^{k,(n+1)^{2}}\right]
$$

with

$$
\begin{gathered}
\mathbf{J}_{\mathbf{2}}=\operatorname{diag}\left[\mathbf{J}_{\mathbf{1}, \mathbf{2}} \cdots \mathbf{J}_{\mathbf{k}, \mathbf{2}} \cdots \mathbf{J}_{\mathbf{n}, \mathbf{2}}\right] \\
\mathbf{a}^{\mathbf{2}}=\left[\mathbf{a}_{\mathbf{1}}^{\mathbf{2}} \cdots \mathbf{a}_{\mathbf{k}}^{\mathbf{2}} \cdots \mathbf{a}_{\mathbf{n}}^{\mathbf{2}}\right]^{T} \\
\mathbf{C}_{\mathbf{2}}=\operatorname{diag}\left[\mathbf{C}_{\mathbf{1}, \mathbf{2}} \cdots \mathbf{C}_{\mathbf{k}, \mathbf{2}} \cdots \mathbf{C}_{\mathbf{n}, \mathbf{2}}\right]
\end{gathered}
$$

Finally, the $n \times 6$ unknown coefficients of the second order approximation contained in the vector $\mathbf{a}^{2}$ are obtained by

$$
\mathbf{a}^{2}=\mathbf{J}_{2}{ }^{-1} \mathbf{C}_{2}
$$

$\mathbf{J}_{\mathbf{2}}$ is a $(6 \times n) \times(6 \times n)$ diagonal matrix. $\mathbf{C}_{\mathbf{2}}$ is a $(6 \times n)$-dimensional vector containing constant terms. All these matrices can be obtained numerically by using the relation (120). As indicated in equations (120) and (124), only the non-linear terms of the second order polynomial expressions of $\mathbf{H}_{2}$ are considered for the determination of the second order coefficients $\mathbf{a}^{2}$ of the centre manifold approximation. We can easily show that though the second order approximation is not sufficient to correctly describe the non-linear dynamic of the original system, the methodology and the centre manifold theory are not in question; this discrepancy reflects only the fact that the approximation of the stable variables $\mathbf{v}_{\mathbf{s}}$ as a power series in $\left(\mathbf{v}_{\mathbf{c}}, \hat{\mu}\right)$ of degree 2 is not sufficient and does not represent a good approximation of the effect of the stables variables $\mathbf{v}_{\mathbf{s}}$ on the centre manifold basis.

\subsubsection{Computational determination of the third order approximation}

In many cases, the second order approximation is not sufficient to obtain a good approximation of the non-linear behaviour of the original system. This is due merely to the fact that the reduced system obtained by using only the second order approximation contains only a small portion of the non-linear expression of the original system. As explained previously, the second order approximation uses only the quadratic terms of the centre variables of the left of (116).

So, the third order approximation can be used to enhance the approximation of the stable variables $\mathbf{v}_{\mathbf{S}}$ as a power series in the centre variables $\left\{\begin{array}{ll}\mathbf{v}_{\mathbf{c}}{ }^{T} & \hat{\mu}\end{array}\right\}^{T}$. We have

$$
\mathbf{v}_{\mathbf{s}}=\mathbf{h}\left(\mathbf{v}_{\mathbf{c}}, \hat{\mu}\right)=\sum_{p=i+j+l=2}^{3} \sum_{j=0}^{p} \sum_{l=0}^{p} \mathbf{a}_{\mathbf{i j} \mathbf{1}} v_{c 1}^{i} v_{c 2}^{j} \hat{\mu}^{l}
$$


This developped expression can be written as follow

$$
\begin{aligned}
\mathbf{v}_{\mathbf{s}}= & \mathbf{h}^{(\mathbf{2})}\left(\mathbf{v}_{\mathbf{c}}, \hat{\mu}\right)=\mathbf{h}^{(\mathbf{1})}\left(\mathbf{v}_{\mathbf{c}}, \hat{\mu}\right)+\mathbf{a}_{\mathbf{3 0 0}} v_{c 1}^{3}+\mathbf{a}_{\mathbf{2 1 0}} v_{c 1}^{2} v_{c 2}+\mathbf{a}_{\mathbf{1 2 0}} v_{c 1} v_{c 2}^{2}+\mathbf{a}_{\mathbf{0 3 0}} v_{c 2}^{2} \\
& +\mathbf{a}_{\mathbf{2 0 1}} v_{c 1}^{2} \hat{\mu}+\mathbf{a}_{\mathbf{1 1 1}} v_{c 1} v_{c 2} \hat{\mu}+\mathbf{a}_{\mathbf{0 2 1}} v_{c 2}^{2} \hat{\mu}+\mathbf{a}_{\mathbf{1 0 2}} v_{c 1} \hat{\mu}^{2}+\mathbf{a}_{\mathbf{0 1 2}} v_{c 2} \hat{\mu}^{2}+\mathbf{a}_{\mathbf{0 0 3}} \hat{\mu}^{3}
\end{aligned}
$$

All the coefficients of the second order have been determined previously. Now, we only need to find the $10 \times n$ coefficients of the third order approximation (with $n$ defining the number of stable variables). This can be obtained by considering the third order terms in the polynomials on both sides in (116):

$$
\begin{array}{r}
D_{\mathbf{v}_{\mathbf{c}}, \hat{\mu}}\left(\mathbf{h}^{(\mathbf{2})}\left(\mathbf{v}_{\mathbf{c}}, \hat{\mu}\right)\right) \mathbf{J}_{\mathbf{c}} \mathbf{v}_{\mathbf{c}}+D_{\mathbf{v}_{\mathbf{c}}, \hat{\mu}}\left(\mathbf{h}^{(\mathbf{1})}\left(\mathbf{v}_{\mathbf{c}}, \hat{\mu}\right)\right) \mathbf{G}_{\mathbf{2}}\left(\mathbf{v}_{\mathbf{c}}, \hat{\mu}\right)-\mathbf{J}_{\mathbf{s}} \mathbf{h}^{(\mathbf{2})}\left(\mathbf{v}_{\mathbf{c}}, \hat{\mu}\right) \\
-\mathbf{H}_{\mathbf{2}}\left(\left[\mathbf{v}_{\mathbf{c}}, \mathbf{0}, \hat{\mu}\right] \otimes\left[\mathbf{v}_{\mathbf{c}}, \mathbf{h}^{(\mathbf{1})}\left(\mathbf{v}_{\mathbf{c}}, \hat{\mu}\right), \hat{\mu}\right]+\left[\mathbf{0}, \mathbf{h}^{(\mathbf{1})}\left(\mathbf{v}_{\mathbf{c}}, \hat{\mu}\right)\right] \otimes\left[\mathbf{v}_{\mathbf{c}}, \mathbf{0}, \hat{\mu}\right]\right)-\mathbf{H}_{\mathbf{3}}\left(\mathbf{v}_{\mathbf{c}}, \hat{\mu}\right)=\mathbf{0}
\end{array}
$$

Then the determination of the coefficients $a_{k, i j l}$ of the third order can be obtained by augmenting the system defined in equation (125):

$$
\left[\begin{array}{cc}
\mathrm{J}_{2} & \mathbf{0} \\
\mathrm{D}_{2,3} & \mathrm{~J}_{3}
\end{array}\right]\left\{\begin{array}{l}
\mathrm{a}^{2} \\
\mathrm{a}^{3}
\end{array}\right\}=\left\{\begin{array}{l}
\mathrm{C}_{2} \\
\mathrm{C}_{3}
\end{array}\right\}
$$

where

$$
\begin{aligned}
& \mathbf{a}^{\mathbf{3}}=\left[\mathbf{a}_{\mathbf{1}}^{\mathbf{3}} \cdots \mathbf{a}_{\mathbf{k}}^{\mathbf{3}} \cdots \mathbf{a}_{\mathbf{n}}^{\mathbf{3}}\right]^{T}
\end{aligned}
$$

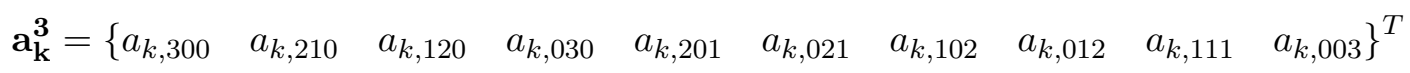

$\mathbf{J}_{2}, \mathbf{C}_{\mathbf{2}}$ and $\mathbf{a}^{\mathbf{2}}$ have been previously defined in equations (126-128). $\mathbf{J}_{\mathbf{3}}$ is a $(10 \times n) \times(10 \times n)$ diagonal matrix and $\mathbf{D}_{\mathbf{2}, 3}$ is a $(10 \times n) \times(6 \times n)$ matrix that defines the contribution of the second order coefficients. $\mathbf{C}_{\mathbf{3}}$ is a $(10 \times n)$ dimensional vector containing constant terms. All these matrices can be obtained numerically by using the relation (132). Then the coefficients $\mathbf{a}^{3}$ of the third order approximation are obtained by

$$
\mathbf{a}^{3}=\mathbf{J}_{3}{ }^{-1}\left(\mathbf{C}_{3}+\mathbf{D}_{2,3} \mathbf{a}^{2}\right)=\mathbf{J}_{3}{ }^{-1}\left(\mathbf{C}_{3}+\mathbf{D}_{2,3} \mathbf{J}_{2}{ }^{-1} \mathbf{C}_{2}\right)
$$

We note that the third order approximation uses the values of the coefficient of the second order approximation. Moreover, a part of the quadratic non-linear terms in centre variables on the left side of (116) contained in $\mathbf{G}_{\boldsymbol{2}}$ defined in equation (113). Cubic terms of the centre variables contained in $\mathbf{H}_{\mathbf{3}}$, defined in equation (113), and quadratic terms of stable variables contained in $\mathbf{H}_{\mathbf{2}}$, defined in equation (113), are used. This clearly indicates that the third order approximation enables consideration of more non-linear terms than the second order approximation. So, it is clear that the third order approximation allows a better approximation than the second order approximation with the contribution of most non-linear terms. As in the case of the second order approximation, if the estimate of the stable variables $\mathbf{v}_{\mathbf{s}}$ in a power series in $\left(\mathbf{v}_{\mathbf{c}}, \hat{\mu}\right)$ of degree 3 is not sufficient and does not represent a good approximation of the contributions of the stables variables on the centre manifold basis, the determination of the $q^{\text {th }}$-order is necessary (with $q \geq 4$ ).

Finally, one of the important points to be noted here is that the number of equations in terms of the unknown coefficients $a_{k, i j l}$ naturally increases in comparison with the second order; this implies that obtaining an analytical expression of these coefficienst $a_{k, i j l}$ may quickly become impossible if the order of the stable variables in power series of centre variables increases.

\subsubsection{Computational determination of the $q^{\text {th }}$ order approximation}

The determination of the $q^{\text {th }}$ order approximation can be generalized by using the same procedure as in Section 3.4.4. The expression of the stable variables $\mathbf{v}_{\mathbf{s}}$ as a power series in $\left(\mathbf{v}_{\mathbf{c}}, \hat{\mu}\right)$ of degree $q$ is defined by

$$
\mathbf{v}_{\mathbf{s}}=\mathbf{h}\left(\mathbf{v}_{\mathbf{c}}, \hat{\mu}\right)=\sum_{p=i+j+l=2}^{q} \sum_{j=0}^{p} \sum_{l=0}^{p} \mathbf{a}_{\mathbf{i j} \mathbf{1}} v_{c 1}^{i} v_{c 2}^{j} \hat{\mu}^{l}
$$

The vector of the $q^{\text {th }}$ order approximation for the $n$ stable variables has the form

$$
\mathbf{a}^{\mathbf{q}}=\left[\mathbf{a}_{\mathbf{1}}^{\mathbf{q}} \cdots \mathbf{a}_{\mathbf{k}}^{\mathbf{q}} \cdots \mathbf{a}_{\mathbf{n}}^{\mathbf{q}}\right]^{T}
$$


It may be determined by solving the following system that is an extension of the system (133) for the $q^{\text {th }}$ order:

$$
\left[\begin{array}{cccccc}
\mathbf{J}_{2} & \mathbf{0} & \cdots & \cdots & \cdots & \mathbf{0} \\
\mathbf{D}_{2,3} & \mathbf{J}_{3} & \ddots & & & \vdots \\
\mathbf{D}_{2,4} & \mathbf{D}_{3,4} & \mathbf{J}_{4} & \ddots & & \vdots \\
\mathbf{D}_{2,5} & \mathbf{D}_{3,5} & \mathbf{D}_{4,5} & \mathbf{J}_{5} & \ddots & \vdots \\
\vdots & \vdots & \vdots & \ddots & \ddots & \mathbf{0} \\
\mathbf{D}_{2, \mathbf{q}} & \mathbf{D}_{3, \mathbf{q}} & \mathbf{D}_{4, \mathbf{q}} & \cdots & \mathbf{D}_{\mathbf{q}-1, \mathbf{q}} & \mathbf{J}_{\mathbf{q}}
\end{array}\right]\left\{\begin{array}{c}
\mathbf{a}^{2} \\
\mathbf{a}^{3} \\
\mathbf{a}^{4} \\
\mathbf{a}^{5} \\
\vdots \\
\mathbf{a}^{\mathbf{q}}
\end{array}\right\}=\left\{\begin{array}{c}
\mathbf{C}_{2} \\
\mathbf{C}_{3} \\
\mathbf{C}_{4} \\
\mathbf{C}_{5} \\
\vdots \\
\mathbf{C}_{\mathbf{q}}
\end{array}\right\}+\left\{\begin{array}{c}
0 \\
\mathbf{0} \\
\mathbf{F}_{4}\left(\mathbf{a}^{2}\right) \\
\mathbf{F}_{5}\left(\mathbf{a}^{2}, \mathbf{a}^{3}\right) \\
\vdots \\
\mathbf{F}_{\mathbf{q}}\left(\mathbf{a}^{2}, \cdots, \mathbf{a}^{\mathbf{q}-2}\right)
\end{array}\right\}
$$

Then the determination of the centre manifold coefficients for each order, and more particularly the $q^{\text {th }}$-order may be obtained successively:

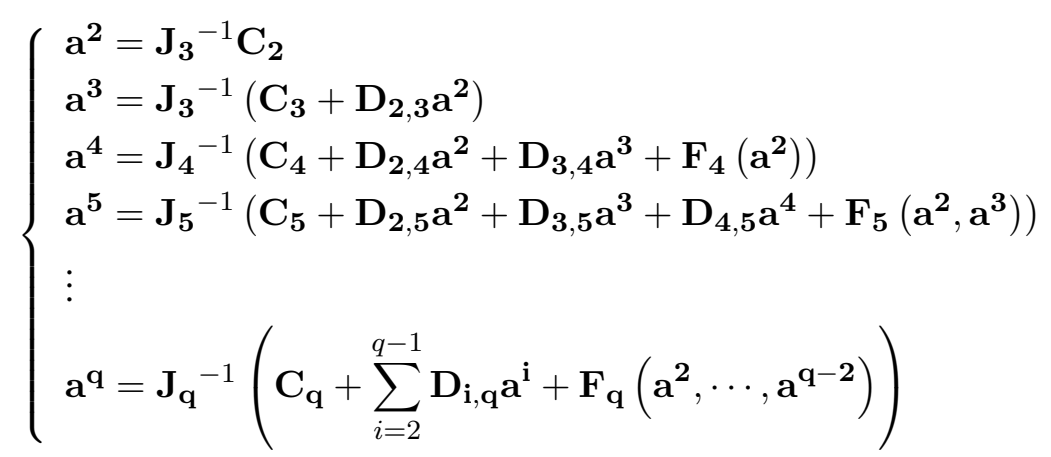

Moreover, the more the higher-order terms are used in order to approximate the stable variables $\mathbf{v}_{\mathbf{s}}$ as a power series of the centre variables $\left(\mathbf{v}_{\mathbf{c}}, \hat{\mu}\right)$, the more the effects of the non-linear terms appear in equations (116) and (139-140) for the determination of coefficients $\mathbf{a}^{\mathbf{q}}$ (with $q>2$ ). As can be shown in equations (139-140), the determination of coefficients $\mathbf{a}^{\mathbf{q}}$ (with $q>2$ ) can be obtained order by order, and there is no need to recalculate the lower-order for a new evaluation of polynomial approximation $\mathbf{v}_{\mathbf{s}}=\mathbf{h}\left(\mathbf{v}_{\mathbf{c}}, \hat{\mu}\right)$. Finally, the analytical expressions for the coefficients $\mathbf{a}^{2}$ and $\mathbf{a}^{3}$ of second-order and third-order polynomial approximations of $\mathbf{v}_{\mathbf{s}}=\mathbf{h}\left(\mathbf{v}_{\mathbf{c}}, \hat{\mu}\right)$ are proposed in Annexe A.

\subsubsection{Numerical estimate of the reduced system via the centre manifold approach}

By using the procedure defined in Section 3.4.5, the original system (112) is reduced to a three-dimensional form near the Hopf bifurcation point $\mu=\mu_{0}+\bar{\mu}$ where $\mu_{0}$ is the Hopf bifurcation point and $\bar{\mu}=\varepsilon \mu_{0}($ with $\varepsilon \ll 1)$ :

$$
\left\{\begin{array}{l}
\dot{\mathbf{v}}_{\mathbf{c}}=\mathbf{J}_{\mathbf{c}}(\mu) \mathbf{v}_{\mathbf{c}}+\mathbf{G}_{\mathbf{2}}\left(\mathbf{v}_{\mathbf{c}}, \mathbf{h}\left(\mathbf{v}_{\mathbf{c}}\right), \mu\right)++\mathbf{G}_{\mathbf{3}}\left(\mathbf{v}_{\mathbf{c}}, \mathbf{h}\left(\mathbf{v}_{\mathbf{c}}\right), \mu\right) \\
\dot{\mu}=0
\end{array}\right.
$$

When the limit cycles are determined near the Hopf bifurcation point (with $\varepsilon$ very small), the expressions $\mathbf{h}\left(\mathbf{v}_{\mathbf{c}}, \bar{\mu}\right)$ may be approximated by the simplified expression $\mathbf{h}\left(\mathbf{v}_{\mathbf{c}}\right)$ with negligible errors; in this case we have $\mu=O\left(\mathbf{v}_{\mathbf{c}}\right)$. The purpose of this consideration is to simplify the expression of the reduced system (141). This approximation thus amounts to the expression of $\mathbf{v}_{\mathbf{s}}$ at the Hopf bifurcation point $\mu_{0}$ with $\mathbf{a}_{\mathbf{i j l}} \equiv \mathbf{0}$ for $l \neq 0$. In other words, it is not necessary, but nonetheless allows the simplification of the expression of (141); this simple extension and simplification to the centre manifold method is useful when dealing with parameterised families of sys-tems. Therefore, the non-linear terms may be approximated by their evaluation at the bifurcation point $\mu_{0}$, provided that none of the leading non-linear terms vanish here; so the approximation $\mathbf{G}_{\mathbf{2}}\left(\mathbf{v}_{\mathbf{c}}, \mathbf{h}\left(\mathbf{v}_{\mathbf{c}}\right), \mu\right)$ and $\mathbf{G}_{\mathbf{3}}\left(\mathbf{v}_{\mathbf{c}}, \mathbf{h}\left(\mathbf{v}_{\mathbf{c}}\right), \mu\right)$ are equivalent to $\mathbf{G}_{\mathbf{2}}\left(\mathbf{v}_{\mathbf{c}}, \mathbf{h}\left(\mathbf{v}_{\mathbf{c}}\right), \mu_{0}\right)$ and $\mathbf{G}_{\mathbf{3}}\left(\mathbf{v}_{\mathbf{c}}, \mathbf{h}\left(\mathbf{v}_{\mathbf{c}}\right), \mu_{0}\right)$ with negligible error due to the fact that $\varepsilon$ is very small.

Finally, an application of the centre manifold theorem to the original system (112) shows that if the equilibrium is 
preserved, then the reduced dynamics at $\mu=\mu_{0}+\bar{\mu}$ is given with small errors by

$$
\left\{\begin{array}{l}
\dot{\mathbf{v}_{\mathbf{c}}}=\mathbf{J}_{\mathbf{c}}(\mu) \mathbf{v}_{\mathbf{c}}+\mathbf{G}_{\mathbf{2}}\left(\mathbf{v}_{\mathbf{c}}, \mathbf{h}\left(\mathbf{v}_{\mathbf{c}}\right), \mu_{0}\right)+\mathbf{G}_{\mathbf{3}}\left(\mathbf{v}_{\mathbf{c}}, \mathbf{h}\left(\mathbf{v}_{\mathbf{c}}\right), \mu_{0}\right) \\
\dot{\mu}=0 \\
\mu=\mu_{0}(1+\varepsilon) \quad(\varepsilon \ll 1) \\
\mathbf{v}_{\mathbf{s}}=\mathbf{h}\left(\mathbf{v}_{\mathbf{c}}\right)=\sum_{p=i+j=2}^{q} \sum_{j=0}^{p} \mathbf{a}_{\mathbf{i j}} v_{c 1}^{i} v_{c 2}^{j} \quad(q \geq 2)
\end{array}\right.
$$

Now, the centre manifold reduction is applied to the system (112). Using an approximation of $\mathbf{h}$ of order 2 causes divergence in the evolutions of limit cycle amplitudes. This is only due to the fact that a polynomial approximation of $\mathbf{h}$ of order 2 is not sufficient to describe with low errors the expressions of the stable variables $\mathbf{v}_{\mathbf{s}}$ and their effects in the reduced system (142). Using an approximation of $\mathbf{h}$ of order 3, 4 or 5 allows good correlations between the 4dimensional original system and the 2-dimensional reduced system, as illustrated in Figures 21 and 22. Consequently, the centre manifold approach is validated; in this case, we reduce the number of equations of the original system (112) from 4 to 2 in order to obtain the simplified system (142) without losing the dynamics of the original system as well as the effects of non-linear terms. Then, this reduced system will be easier to study than the original one. Moreover, one of the most important points is the determination of polynomial approximations and the estimate of power that defines the expressions of stable variables versus centre variables: the more complex the expressions of the stable variables $\mathbf{v}_{\mathbf{s}}$ and the associated polynomial approximation $\mathbf{h}$ are, the more interesting the estimate of the reduced system is, allowing us to obtain an estimate of the non-linear dynamics of (142) near (112), as illustrated in Figures 21 and 22. However, the more complex the expressions of the $\mathbf{v}_{\mathbf{s}}$ and $\mathbf{h}$ are, the more costly and time consuming the computations are.

Finally, it may be noted that the more complex the non-linear system is, with many degrees-of-freedom, the more interesting the centre manifold approach is, allowing us to reduce the original system from a m-dimensional form to a lower-dimensional form and to save time.

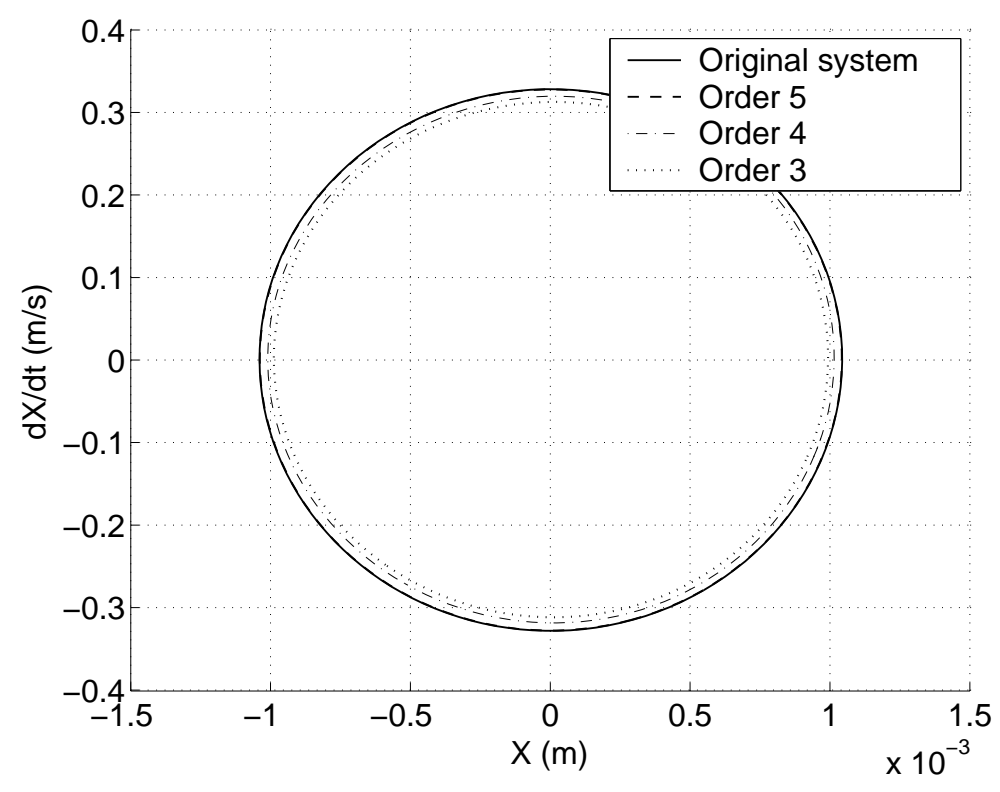

(a) Limit cycle $(X, \dot{X})$

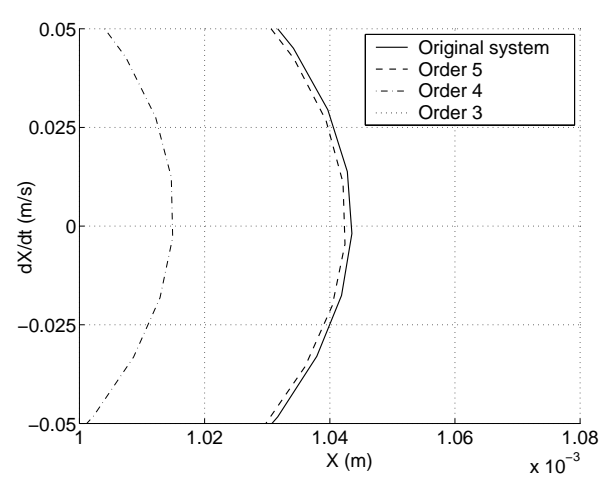

(b) Zoom

Figure 21: Limit cycle $(X, \dot{X})$ by using the center manifold approach for $\mu=1.001 \mu_{0}$ 


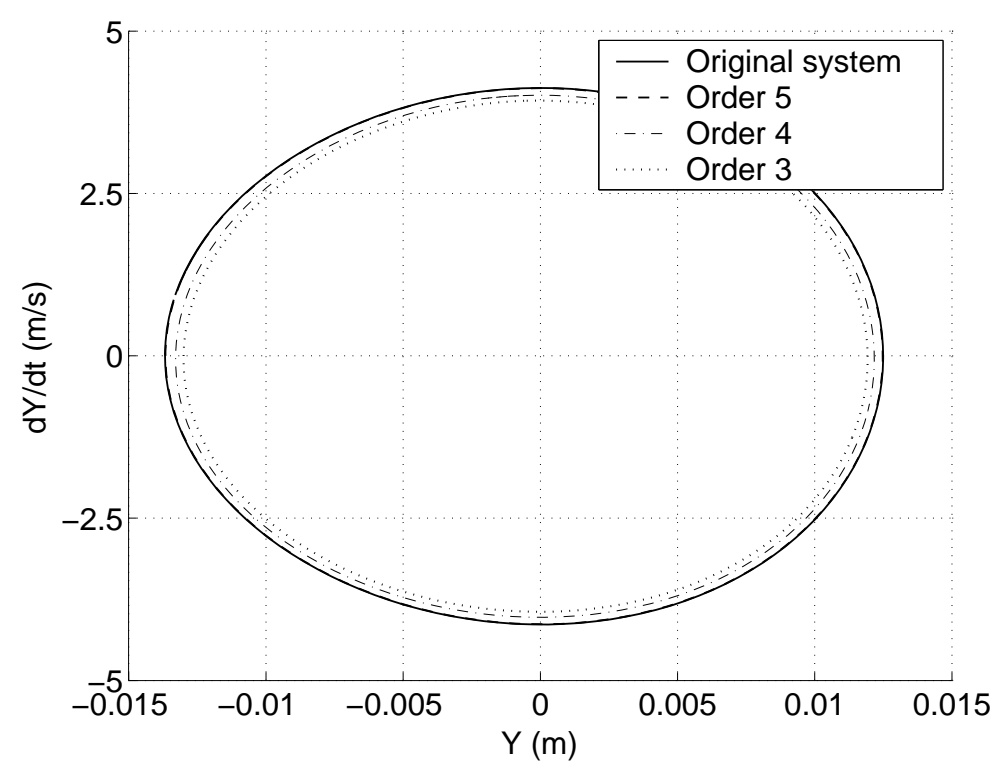

(a) Limit cycle $(Y, \dot{Y})$

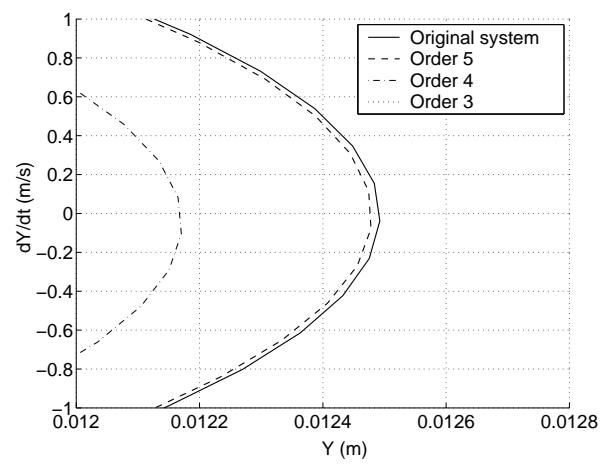

(b) Zoom

Figure 22: Limit cycle $(Y, \dot{Y})$ by using the center manifold approach for $\mu=1.001 \mu_{0}$

\subsection{Simplified non-linear system via the multivariable approximants}

As indicated in the previous section, the centre manifold approach reduces a non-linear system with many degrees of freedom to a lower-dimensional form near the Hopf bifurcation point. However, it may be observed that this nonlinear method swaps a non-linear system with a high number of degrees of freedom with a "simple" non-linearity for one with fewer degree of freedom, but a more complicated non-linearity. Moreover, the formal centre manifold approximation is not difficult to determine; but, obtaining the coefficients associated which each term of the stable variables may pose particularly serious difficulties. This is why the sole use of the centre manifold approach is not very convenient, requiring a great deal of labour, especially for the calculation of the coefficients defined previously. Due to the fact that the centre manifold can have complicated non-linear terms, further non-linear methods are applied after the centre manifold reduction. In this study, the rational fractional approximants are used; the interest of these multivariable approximants is that they require fewer terms than the associated Taylor series in order to obtain an accurate approximation of a non-linear function: they allow the computation of an accurate approximation of the nonlinear function $f(\mathbf{x})$ even at values of $\mathbf{x}$ for which the Taylor series of $f$ diverge. We will consider this last property of the rational fractional approximants in this paper in order to augment the domain of validity of the series previously obtained by using the centre manifold approach with a $q^{t h}$-order polynomial approximation $\mathbf{h}$ of the stable variables $\mathbf{v}_{\mathbf{s}}$ in the centre variables $\left(\mathbf{v}_{\mathbf{c}}\right)$. This property may be very interesting in regard to the appropriate order of the centre manifold polynomial approximation $\mathbf{h}$ needed to obtain a good correlation between the reduced system via the centre manifold method and the original system.

Moreover, the objective is to approximate the non-linear terms by using rational polynomial approximants: the use of the rational approximants allows us to simplify the non-linear system and to obtain the non-linear dynamical responses of the system more easily and rapidly.

\subsubsection{Transformation from the centre manifold form to the fractional approximants form}

Before applying the fractional rational approximants, the non-linear reduced system expressed in the centre manifold basis is transformed in a power series $v_{c 1}$ and $v_{c 2}$. The expressions of $\mathbf{G}_{\mathbf{2}}\left(\mathbf{v}_{\mathbf{c}}, \mathbf{h}\left(\mathbf{v}_{\mathbf{c}}\right)\right)$ and $\mathbf{G}_{\mathbf{3}}\left(\mathbf{v}_{\mathbf{c}}, \mathbf{h}\left(\mathbf{v}_{\mathbf{c}}\right)\right)$ can be 
developped as follows

$$
\begin{aligned}
& \mathbf{G}_{\mathbf{2}}\left(\mathbf{v}_{\mathbf{c}}, \mathbf{h}\left(\mathbf{v}_{\mathbf{c}}\right)\right)=\left\{\begin{array}{c}
\sum_{p=i+j=2}^{2 m} \sum_{j=0}^{p} \varphi_{1, i j} v_{c 1}^{i} v_{c 2}^{j} \\
\sum_{p=i+j=2}^{2 m} \sum_{j=0}^{p} \varphi_{2, i j} v_{c 1}^{i} v_{c 2}^{j}
\end{array}\right\} \\
& \mathbf{G}_{\mathbf{3}}\left(\mathbf{v}_{\mathbf{c}}, \mathbf{h}\left(\mathbf{v}_{\mathbf{c}}\right)\right)=\left\{\begin{array}{c}
\sum_{p=i+j=2}^{3 m} \sum_{j=0}^{p} \gamma_{1, i j} v_{c 1}^{i} v_{c 2}^{j} \\
\sum_{p=i+j=2}^{3 m} \sum_{j=0}^{p} \gamma_{2, i j} v_{c 1}^{i} v_{c 2}^{j}
\end{array}\right\}
\end{aligned}
$$

where $\varphi_{1, i j}$ and $\varphi_{2, i j}$ are the constant coefficients from the quadratic expressions of (142), and $\gamma_{1, i j}$ et $\gamma_{2, i j}$ are the constant coefficients from the cubic expressions of (142).

By considering (142-144), the non-linear system (142) can be rewritten as follows:

$$
\left\{\begin{array}{l}
\dot{v_{c 1}}=\sum_{i=0}^{3 m} \sum_{\substack{j=0 \\
1 \leq i+j \leq 3 m}}^{3 m} c_{1, i j} v_{c 1}^{i} v_{c 2}^{j} \\
\dot{v_{c 2}}=\sum_{i=0}^{3 m} \sum_{\substack{j=0 \\
1 \leq i+j \leq 3 m}}^{3 m} c_{2, i j} v_{c 1}^{i} v_{c 2}^{j}
\end{array}\right.
$$

where $c_{1, i j}$ et $c_{2, i j}$ are the coefficients associated with the power $v_{c 1}^{i} v_{c 2}^{j}(i+j \geq 1)$ for $v_{c 1}$ and $v_{c 2}$, respectively. Finally, the reduced system (142) may be rewritten as follows

$$
\left\{\begin{array}{l}
\dot{v_{c k}}=f_{k}\left(v_{c 1}, v_{c 2}\right)=\sum_{i=0}^{3 m} \sum_{\substack{j=0 \\
1 \leq i+j \leq 3 m}}^{3 m} c_{k, i j} v_{c 1}^{i} v_{c 2}^{j} \quad(1 \leq k \leq 2) \\
\dot{\mu}=0 \\
\mu=\mu_{0}(1+\varepsilon) \quad(\varepsilon \ll 1)
\end{array}\right.
$$

We now consider the Symmetric-Off-Diagonal (SOD) approximants $\left(m_{1}=m_{2}=M\right.$ and $\left.n_{1}=n_{2}=N\right)$ to $f_{k}\left(v_{c 1}, v_{c 2}\right)$. The choice of the SOD-approximants instead of the GOD-approximants is only due to the fact that the two centre variables $v_{c 1}$ and $v_{c 2}$ are assumed to be of the same order and then to have the same effect for the nonlinear dynamical befaviour of the system. Moreover, the SOD-approximants are preferred instead of the Chisholm approximants because they enable consideration of more complex expressions of the non-linear approximation. As explained in Section 2.4 the SOD approximants $[M / N]_{f_{k}}\left(v_{c 1}, v_{c 2}\right)$ associated with the system (146) have the general form

$$
[M / N]_{f_{k}}\left(v_{c 1}, v_{c 2}\right)=\frac{\sum_{\alpha=0}^{M} \sum_{\beta=0}^{M} n_{k, \alpha \beta} v_{c 1}^{\alpha} v_{c 2}^{\beta}}{\sum_{\alpha=0}^{N} \sum_{\beta=0}^{N} d_{k, \alpha \beta} v_{c 1}^{\alpha} v_{c 2}^{\beta}} \quad(\text { for } \quad 1 \leq k \leq 2)
$$

where $[M / N]_{f_{k}}\left(v_{c 1}, v_{c 2}\right)$ is the rational function for the $k^{t h}$-variable $v_{c k}$ with a numerator of degree $M$ and a denominator of degree $N$.

\subsubsection{Computational determination of the fractional approximants coefficients}

As explained in Section 2.4, the SOD approximants $[M / N]_{f_{k}}$ satisfies the $k$ relations

$$
\sum_{i=0}^{3 m} \sum_{\substack{j=0 \\ 2 \leq i+j \leq 3 m}}^{3 m} c_{k, i j} v_{c 1}^{i} v_{c 2}^{j}=[M / N]_{f_{k}}\left(v_{c 1}, v_{c 2}\right)+\sum_{\alpha=0}^{\infty} \sum_{\beta=0}^{\infty} e_{k, \alpha \beta} v_{c 1}^{\alpha} v_{c 2}^{\beta} \quad(\text { for } \quad 1 \leq k \leq 2)
$$


where as many coefficients $e_{k, \alpha \beta}$ as possible are equal to zero. Then, the coefficients $n_{k, \alpha \beta}$ (for $1 \leq k \leq 2,0 \leq$ $\alpha \leq M$, and $0 \leq \alpha \leq M$ ) and $d_{k, \alpha \beta}$ (for $1 \leq k \leq 2,0 \leq \alpha \leq N$, and $0 \leq \alpha \leq N$ ) are determined by matching the coefficients for identical power $v_{c 1}^{\alpha} v_{c 2}^{\beta}$, as indicated in equations (58-60). The equations obtained by matching the coefficients in (148) are

$$
\begin{gathered}
d_{k, 00}=1 \\
\sum_{i=0}^{\alpha} \sum_{j=0}^{\beta} d_{k, i j} c_{k, \alpha-i, \beta-j}=n_{k, \alpha \beta} \quad 0 \leq \alpha \leq M, \quad 0 \leq \beta \leq M \\
\sum_{i=0}^{\alpha} \sum_{j=0}^{N} d_{k, i j} c_{k, \alpha-i, \beta-j}=0 \quad 0 \leq \alpha<N, \quad M<\beta \leq M+N-\alpha \\
\sum_{i=0}^{N} \sum_{j=0}^{\beta} d_{k, i j} c_{k, \alpha-i, \beta-j}=0 \quad M \leq \alpha<M+N-\beta, \quad 0 \leq \beta<N \\
\sum_{i=0}^{\nu} \sum_{j=0}^{N} d_{k, i j} c_{k, \nu-i, M+N+1-\nu-j}+d_{k, j i} c_{k, M+N+1-\nu-i, \nu-j}=0 \quad 1 \leq \nu \leq N
\end{gathered}
$$

with $1 \leq k \leq 2$. After normalizing $d_{k, 00}$ to unity as indicated in (149), there are $k \times\left((M+1)^{2}+(N+1)^{2}-1\right)$ unknown coefficients in equations (150-153). The first step is the determination of the $k \times\left((N+1)^{2}-1\right)$ unknown coefficients $d_{k, i j}$. It is useful to introduce the lattice space diagram to indicate the regions in which the terms $v_{c 1}^{\alpha} v_{c 2}^{\beta}$ are to be matched, as illustrated in Figure 23. $k \times(N(N+1) / 2)$ equations arise from each of (151) and (152) which are obtained by matching terms of the two triangular regions $S_{3}$. Now, $k \times N$ equations arise from (153) obtained by equating to zero the sums of the coefficients of the pairs $v_{c 1}^{\alpha} v_{c 2}^{\beta}$ and $v_{c 1}^{\beta} v_{c 2}^{\alpha}$. These pairs are indicated in the regions $S_{4}$ by the the two associated points $A_{k}$ (with $\left.k=1,2, \ldots, N\right)$. Finally, the $k \times\left((N+1)^{2}-1\right)$ coefficients $d_{k, i j}$ can be achieved by solving the $k \times\left((N+1)^{2}-1\right)$ linear equations (151-153). Next, the $k \times\left((M+1)^{2}\right)$ coefficients $n_{k, i j}$ may be found by directly solving the equations (150); the associated terms $v_{c 1}^{\alpha} v_{c 2}^{\beta}$ are in the regions $S_{1} \bigcup S_{2} \cup S_{5}$. In conclusion, It is easy to obtain the unknowm coefficients $n_{k, i j}$ and $d_{k, i j}$ from equations (149-153). However, the resolution of the $k \times\left((M+1)^{2}+(N+1)^{2}\right)$ linear equations may be both time consuming and costly to perform, and require a very large storage space. However, it is possible to apply a special process, called the " prong method", to rapidly compute the coefficients by taking the equations (149-153) in a special order. This computational process reduces the calculation of all the coefficients to linear algebra with a lower triangular block by block resolution which greatly simplifies the determination of the coefficients $n_{k, i j}$ and $d_{k, i j}$.

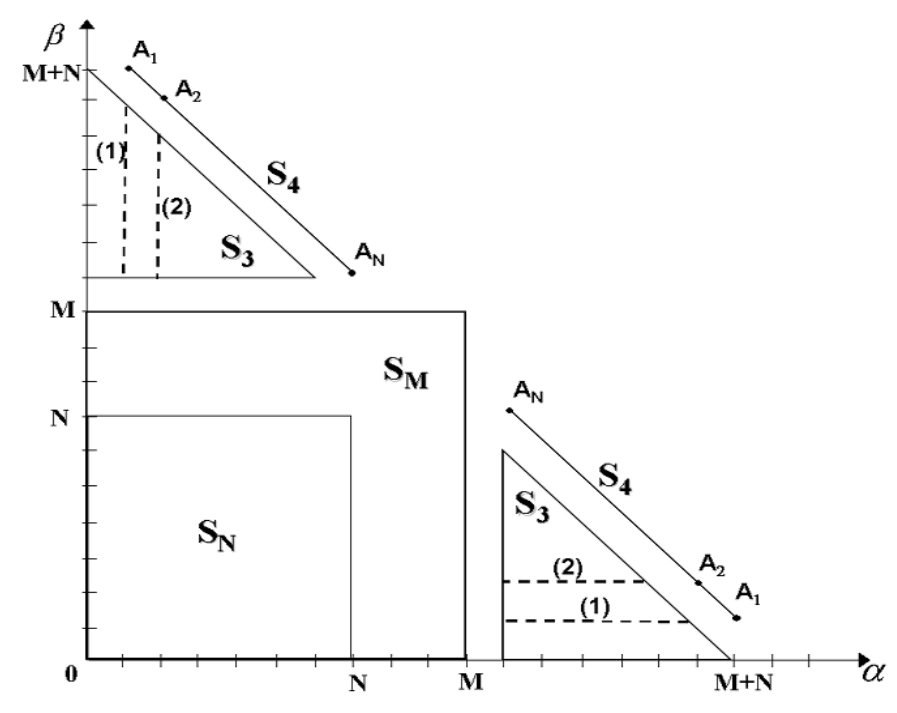

Figure 23: Lattice space for the SOD approximants 
The first step in the "prong method" ([3], [74] and [75]) consists of determining the denominator coefficients $d_{k, i j}$. As explaind previously, the determination of this coefficients can be achieved by considering the pairs of two regions $S_{3}$ and $S_{4}$. However, it may be observed that the $k \times(N+1)$ coefficients $d_{k, i, 0}$ (with $\left.0 \leq i \leq N\right)$ are matched by considering the segment of the lattice space $(M+1 \leq \alpha \leq M+N, \beta=0)$. These equations written in matrix form become

$$
\left[\begin{array}{ccc}
c_{k, M-N+1,0} & \cdots & c_{k, M+1,0} \\
\vdots & & \vdots \\
c_{k, M, 0} & \cdots & c_{k, M+N, 0}
\end{array}\right]\left\{\begin{array}{c}
d_{k, N, 0} \\
\vdots \\
d_{k, 0,0}
\end{array}\right\}=\left\{\begin{array}{c}
0 \\
\vdots \\
0
\end{array}\right\}
$$

Similarly, the matching of the $k \times(N+1)$ coefficients $d_{k, 0, j}$ (with $\left.0 \leq j \leq N\right)$ located on the segment $(\alpha=0, M+1 \leq \beta \leq M+$ produces

$$
\left[\begin{array}{ccc}
c_{k, 0, M-N+1} & \cdots & c_{k, 0, M+1} \\
\vdots & & \vdots \\
c_{k, 0, M} & \cdots & c_{k, 0, M+N}
\end{array}\right]\left\{\begin{array}{c}
d_{k, 0, N} \\
\vdots \\
d_{k, 0,0}
\end{array}\right\}=\left\{\begin{array}{c}
0 \\
\vdots \\
0
\end{array}\right\}
$$

By normalizing equation $d_{k, 0,0}=1$, the two systems (154) and (155) may be rewritten in the following form

$$
\left[\begin{array}{ccccccc}
c_{k, 0, M-N+1} & \cdots & c_{k, 0, M} & 0 & \cdots & 0 & c_{k, 0, M+1} \\
\vdots & & \vdots & \vdots & & \vdots & \vdots \\
c_{k, 0, M} & \cdots & c_{k, 0, M+N-1} & 0 & \cdots & 0 & c_{k, 0, M+N} \\
0 & \cdots & 0 & c_{k, M-N+1,0} & \cdots & c_{k, M, 0} & c_{k, M+1,0} \\
\vdots & & \vdots & \vdots & & \vdots & \vdots \\
0 & \cdots & 0 & c_{k, M, 0} & \cdots & c_{k, M+N-1,0} & c_{k, M+N, 0} \\
0 & \cdots & 0 & 0 & \cdots & 0 & 1
\end{array}\right]\left\{\begin{array}{c}
d_{k, 0, N} \\
\vdots \\
d_{k, 0,1} \\
d_{k, N, 0} \\
\vdots \\
d_{k, 1,0} \\
d_{k, 0,0}
\end{array}\right\}=\left\{\begin{array}{c}
0 \\
\vdots \\
0 \\
0 \\
\vdots \\
0 \\
1
\end{array}\right\}
$$

The previous system (156) may be written in the compact form

$$
\mathbf{A}_{\mathrm{k}, 0} \mathbf{d}_{\mathrm{k}, 0}=\mathbf{u}_{\mathrm{k}}
$$

where $\mathbf{d}_{\mathbf{k}, \mathbf{0}}$ defines the $(2 \times N+1)$-dimensional vector of the coefficients $d_{k, 0,0}, d_{k, 0, i}$ and $d_{k, i, 0}$ (with $\left.1 \leq i \leq N\right)$. $\mathbf{u}_{\mathbf{k}}$ is a column vector of dimension $2 \times N+1$ with unity in the $(2 \times N+1)^{t h}$ place and zeros elsewhere. Finally, the $k^{\text {th }}$ vector $\mathbf{d}_{\mathbf{k}, \mathbf{0}}$ may be obtained:

$$
\mathbf{d}_{\mathbf{k}, \mathbf{0}}=\mathbf{A}_{\mathbf{k}, \mathbf{0}}{ }^{-1} \mathbf{u}_{\mathbf{k}}
$$

Next, the $k \times(2 \times N-1)$ coefficients $d_{k, 1,1}, d_{k, i, 1}$ and $d_{k, 1, i}$ (with $\left.2 \leq i \leq N\right)$ are obtained by matching terms on the segments of the lattice space $(M+1 \leq \alpha \leq M+N-1, \beta=1)$ and $(\alpha=1, M+1 \leq \beta \leq M+N-1)$ (defined in the two regions $S_{3}$ by the lines marked (1)), and on the symmetrized linked pair of points $(1, M+N)$ and $(M+N, 1)$ (defined by the two points $\left.A_{1}\right)$. The equations of the determination of these coefficients may be written in the matrix form

$$
\mathbf{B}_{\mathrm{k}, 11} \mathbf{d}_{\mathrm{k}, 0}+\mathbf{A}_{\mathrm{k}, 1} \mathbf{d}_{\mathrm{k}, 1}=\mathbf{0}
$$

where $\mathbf{d}_{\mathbf{k}, \mathbf{1}}$ defines the $(2 \times(N-1)+1)$-dimensional vector

$$
\mathbf{d}_{\mathbf{k}, \mathbf{1}}=\left\{d_{k, 1, N} \cdots d_{k, 1,2} \quad d_{k, N, 1} \cdots d_{k, 2,1} \quad d_{k, 1,1}\right\}^{T}
$$

and

$$
\mathbf{A}_{\mathbf{k}, \mathbf{1}}=\left[\begin{array}{ccccccc}
c_{k, 0, M-N+1} & \cdots & c_{k, 0, M-1} & 0 & \cdots & 0 & c_{k, 0, M} \\
\vdots & & \vdots & \vdots & & \vdots & \vdots \\
c_{k, 0, M-1} & \cdots & c_{k, 0, M+N-3} & 0 & \cdots & 0 & c_{k, 0, M+N-2} \\
0 & \cdots & 0 & c_{k, M-N+1,0} & \cdots & c_{k, M-1,0} & c_{k, M, 0} \\
\vdots & & \vdots & \vdots & & \vdots & \vdots \\
0 & \cdots & 0 & c_{k, M-1,0} & \cdots & c_{k, M+N-3,0} & c_{k, M+N-2,0} \\
c_{k, 0, M} & \cdots & c_{k, 0, M+N-2} & c_{k, M, 0} & \cdots & c_{k, M+N-2,0} & c_{k, M+N-1,0}+c_{k, 0, M+N-1}
\end{array}\right]
$$


$\mathbf{B}_{\mathbf{k}, \mathbf{1 1}} \mathbf{d}_{\mathbf{k}, \mathbf{0}}$ defines a $(2 \times N+1)$-dimensional vector of known quantity. Finally, the $k^{\text {th }}$ vector $\mathbf{d}_{\mathbf{k}, \mathbf{1}}$ may be obtained:

$$
\mathbf{d}_{\mathrm{k}, \mathbf{1}}=-\mathbf{A}_{\mathrm{k}, \mathbf{1}}{ }^{-1} \mathbf{B}_{\mathrm{k}, \mathbf{1 1}} \mathbf{d}_{\mathrm{k}, \mathbf{0}}
$$

By using an iterative process, the overall system of the equations involving the determination of the $\mathbf{d}_{\mathbf{k}, \mathbf{p}}$ vectors (with $1 \leq p \leq N)$ is defined by

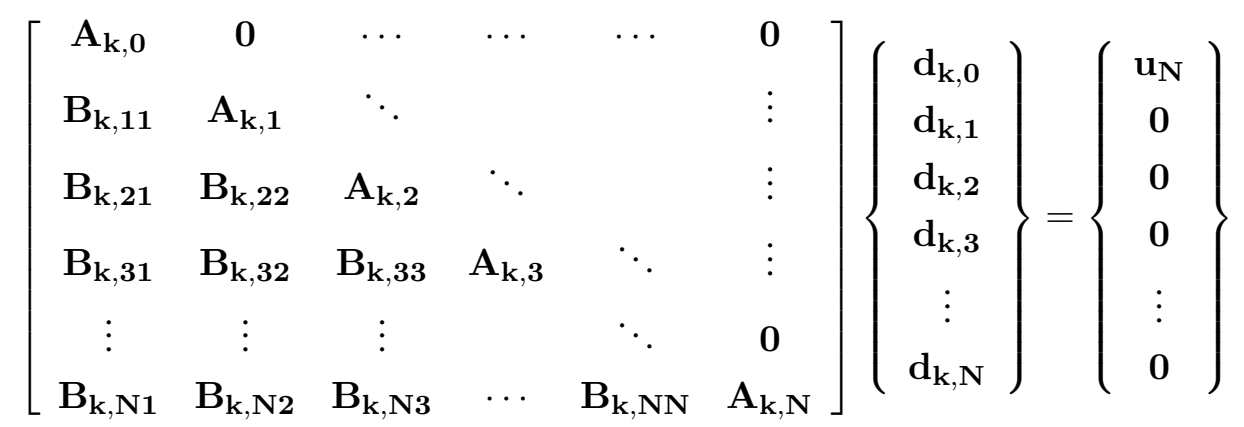

The matrices $\mathbf{B}_{\mathbf{k}, \mathbf{i j}}$ (with $0 \leq i \leq N$ and $\left.1 \leq i \leq N\right)$ are known. The $(2 N-2 i+1)$-dimensional vector $\mathbf{d}_{\mathbf{k}, \mathbf{i}}$ (with $1 \leq i \leq N)$ may be obtained by a block by block inversion process.

These equations written in matrix form become

$$
\left\{\begin{array}{l}
\mathbf{d}_{\mathbf{k}, \mathbf{0}}=\mathbf{A}_{\mathbf{k}, \mathbf{0}}{ }^{-1} \mathbf{u}_{\mathbf{N}} \\
\mathbf{d}_{\mathbf{k}, \mathbf{1}}=\mathbf{A}_{\mathbf{k}, \mathbf{1}}{ }^{-1}\left(-\mathbf{B}_{\mathbf{k}, \mathbf{1 1}} \mathbf{d}_{\mathbf{k}, \mathbf{0}}\right) \\
\mathbf{d}_{\mathbf{k}, \mathbf{2}}=\mathbf{A}_{\mathbf{k}, \mathbf{2}}{ }^{-1}\left(-\mathbf{B}_{\mathbf{k}, \mathbf{2 1}} \mathbf{d}_{\mathbf{k}, \mathbf{0}}-\mathbf{B}_{\mathbf{k}, \mathbf{2 2}} \mathbf{d}_{\mathbf{k}, \mathbf{1}}\right) \\
\mathbf{d}_{\mathbf{k}, \mathbf{3}}=\mathbf{A}_{\mathbf{k}, \mathbf{3}}{ }^{-1}\left(-\mathbf{B}_{\mathbf{k}, \mathbf{3 1}} \mathbf{d}_{\mathbf{k}, \mathbf{0}}-\mathbf{B}_{\mathbf{k}, \mathbf{3 2}} \mathbf{d}_{\mathbf{k}, \mathbf{1}}-\mathbf{B}_{\mathbf{k}, \mathbf{3 3}} \mathbf{d}_{\mathbf{k}, \mathbf{2}}\right) \\
\vdots \\
\mathbf{d}_{\mathbf{k}, \mathbf{N}}=\mathbf{A}_{\mathbf{k}, \mathbf{N}}{ }^{-1}\left(-\sum_{i=1}^{N} \mathbf{B}_{\mathbf{k}, \mathbf{N i}} \mathbf{d}_{\mathbf{k}, \mathbf{i}-\mathbf{1}}\right)
\end{array}\right.
$$

This block by block process has been termed the "'prong method"'([74] and [75]). It may be observed that this process takes the equations defined by (149) and (138-153) in a special order so that the computation of the coefficients $d_{k, i j}$ are simplified.

Finally, the $k \times(M+1)^{2}$ numerator coefficients $n_{k, i j}$ can be found easily by considering the relations (150). It may be observed that the determination of these coefficients can be achieved by considering the succesive orders:

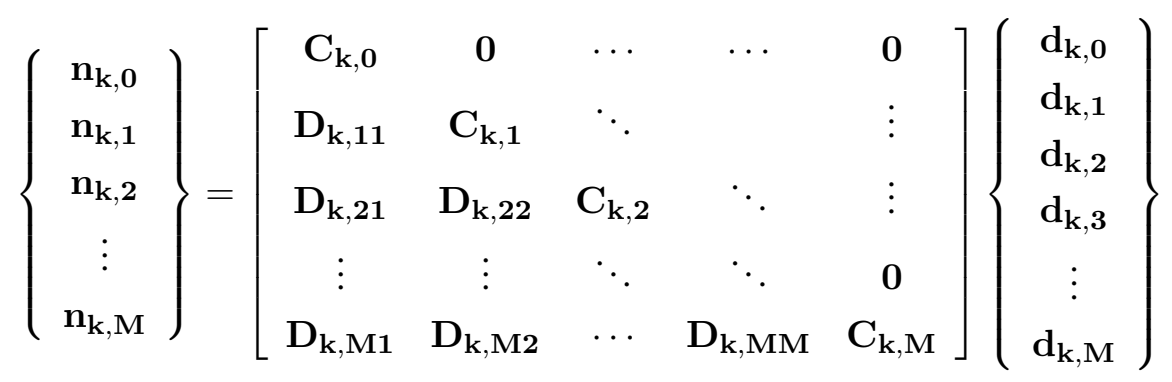

All the matrices $\mathbf{C}_{\mathbf{k}, \mathbf{i}}$ (with $0 \leq i \leq M$ ) and $\mathbf{D}_{\mathbf{k}, \mathbf{j l}}$ (with $1 \leq j \leq M$ and $1 \leq l \leq M$ ) are known and the vector $\mathbf{d}_{\mathbf{k}, \mathbf{i}}$ (with $0 \leq i \leq N$ ) have been obtained previously by using the "prong method". Finally, the vector coefficients $\mathbf{n}_{\mathbf{k}, \mathbf{i}}$ (with $0 \leq i \leq M$ ) are defined by

$$
\left\{\begin{array}{l}
\mathbf{n}_{\mathbf{k}, \mathbf{0}}=\mathbf{C}_{\mathbf{k}, \mathbf{0}} \mathbf{d}_{\mathbf{k}, \mathbf{0}} \\
\mathbf{n}_{\mathbf{k}, \mathbf{1}}=\mathbf{C}_{\mathbf{k}, \mathbf{1}} \mathbf{d}_{\mathbf{k}, \mathbf{1}}+\mathbf{D}_{\mathbf{k}, \mathbf{1 1}} \mathbf{d}_{\mathbf{k}, \mathbf{0}} \\
\mathbf{n}_{\mathbf{k}, \mathbf{2}}=\mathbf{C}_{\mathbf{k}, \mathbf{2}} \mathbf{d}_{\mathbf{k}, \mathbf{2}}+\mathbf{D}_{\mathbf{k}, \mathbf{2 1}} \mathbf{d}_{\mathbf{k}, \mathbf{0}}+\mathbf{D}_{\mathbf{k}, \mathbf{2} 2} \mathbf{d}_{\mathbf{k}, \mathbf{1}} \\
\vdots \\
\mathbf{n}_{\mathbf{k}, \mathbf{M}}=\mathbf{C}_{\mathbf{k}, \mathbf{M}} \mathbf{d}_{\mathbf{k}, \mathbf{M}}+\sum_{i=1}^{M} \mathbf{D}_{\mathbf{k}, \mathbf{M i}} \mathbf{d}_{\mathbf{k}, \mathbf{i}-\mathbf{1}}
\end{array}\right.
$$




\subsubsection{Numerical estimate of the reduced and simplified system via the fractional approximants}

The Symmetric-Off-Diagonal (SOD) approximants and the computational technique previously explained are applied in order to simplify the non-linear expression of (146), that is a power series in $\left(v_{c 1}, v_{c 2}\right)$ of degree 15 , without constant terms. The system (146) can be rewritten in SOD approximants by

$$
\left\{\begin{array}{c}
\dot{v_{c 1}} \\
\dot{v_{c 2}}
\end{array}\right\}=\left\{\begin{array}{c}
\sum_{i=0}^{3 m} \sum_{\substack{j=0 \\
1 \leq i+j \leq 3 m}}^{3 m} c_{1, i j}(\mu) v_{c 1}^{i} v_{c 2}^{j} \\
\sum_{i=0}^{3 m} \sum_{\substack{j=0 \\
1 \leq i+j \leq 3 m}}^{3 m} c_{2, i j}(\mu) v_{c 1}^{i} v_{c 2}^{j}
\end{array}\right\}=\left\{\begin{array}{c}
\sum_{\alpha=0}^{M} \sum_{\beta=0}^{M} n_{1, \alpha \beta}(\mu) v_{c 1}^{\alpha} v_{c 2}^{\beta} \\
\sum_{\alpha=0}^{N} \sum_{\beta=0}^{N} d_{1, \alpha \beta}(\mu) v_{c 1}^{\alpha} v_{c 2}^{\beta} \\
{[M / N]_{f_{1}\left(v_{c 1}, v_{c 2}\right)}=\frac{\sum_{\alpha=0}^{M} \sum_{\beta=0}^{M} n_{2, \alpha \beta}(\mu) v_{c 1}^{\alpha} v_{c 2}^{\beta}}{\sum_{\alpha=0}^{N} \sum_{\beta=0}^{N} d_{2, \alpha \beta}(\mu) v_{c 1}^{\alpha} v_{c 2}^{\beta}}}
\end{array}\right\}
$$

where all the coefficients $d_{1, \alpha \beta}, d_{2, \alpha \beta}$ (for $\left.i, j=0,1, \ldots, N\right)$ and $n_{1, \alpha \beta}, n_{2, \alpha \beta}($ for $i, j=0,1, \ldots, M$ ) are estimated by using the " prong method" [75] defined in the previous Section 3.5.2.

In order to obtain a good estimate of the non-linear behaviour of the system (167), the $[5 / 4]_{f_{k}\left(v_{c 1}, v_{c 2}\right)}$ (with $1 \leq k \leq 2$ ) approximants are applied. An $[M / N]_{f_{k}\left(v_{c 1}, v_{c 2}\right)}$ approximation with $M \leq 4$ and $N \leq 4$ appears to be insufficient to describe the dynamics of the original system: effectively, in some cases, computations diverge since the non-linearities retained are not sufficient, and in other cases, the limit cycle amplitudes obtained are not acceptable due to the same reasons. An $[M / N]_{f_{k}\left(v_{c 1}, v_{c 2}\right)}$ approximation with $M \geq 5$ and $N \geq 4$ gives a good correlation with the original non-linear system as illustrated in Figures 24 and 25. We may note in fact that the results from the SOD approximants and the " "exact"" solution obtained by using the $4^{\text {th }}$ Runge-Kutta process are hardly distinguishable.

Moreover, one of the interests of multivariable approximants is that they require fewer terms than the Taylor series for obtaining an accurate approximation of the limit cycle amplitudes. In this case, it may be observed that the centre manifold approach requires at least order 5 to have the same estimate of the limit cycles as the $[5 / 4]_{f_{k}\left(v_{c 1}, v_{c 2}\right)}$ fractional approximants. So, the non-linear terms become a power series of degree 15 in which all terms are relevant when considering the centre manifold approach. In the case of the fractional approximants, fewer terms are used to obtain the same solution. Moreover, the determination of limit cycle amplitudes by the integration of the differentialalgebraic equations of the system is faster using the multivariable approximants. All these properties of the fractional approximants will be discussed later and analysed in detail.

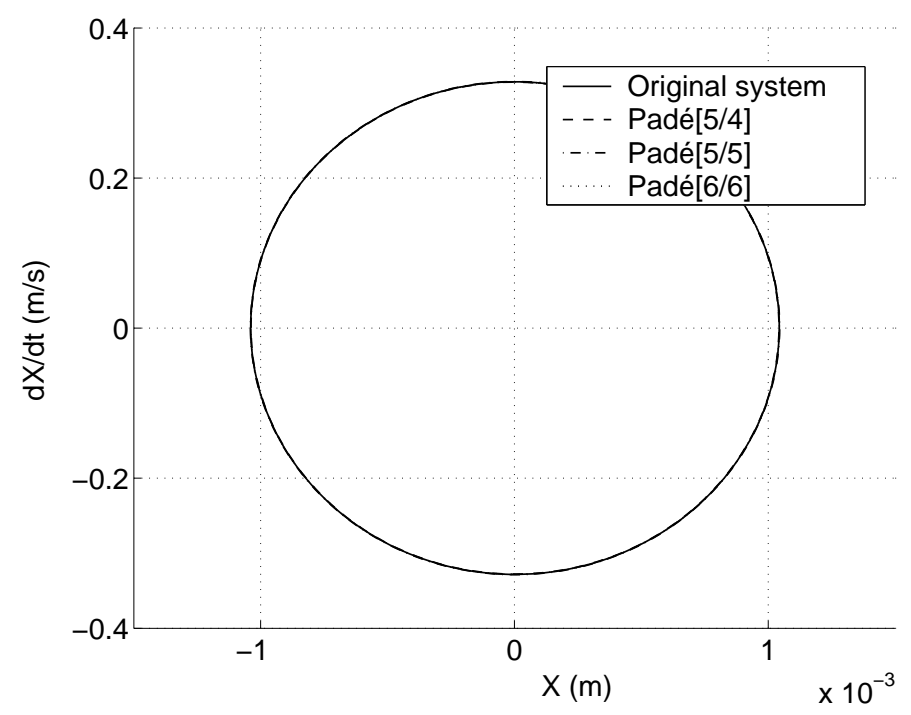

(a) Limit cycles $(X, \dot{X})$

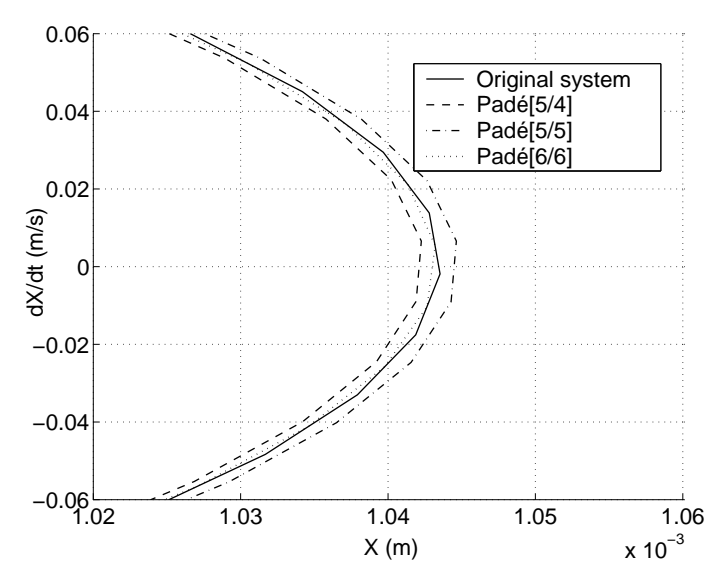

(b) Zoom

Figure 24: Limit cycles $(X, \dot{X})$ for $\mu=1.001 \mu_{0}$ by using the fractional approximants 


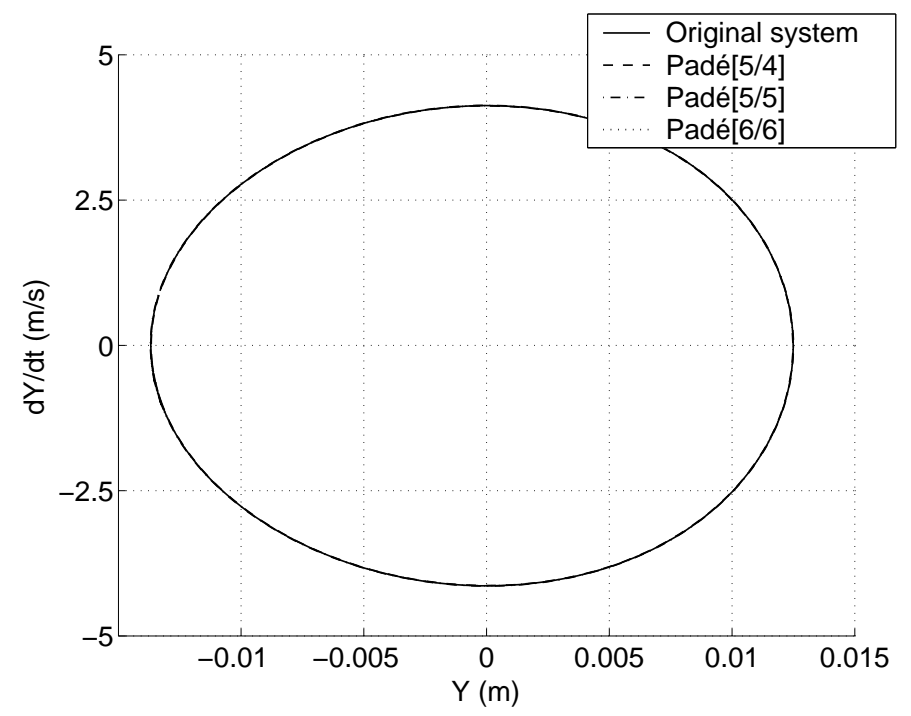

(a) Limit cycles $(Y, \dot{Y})$

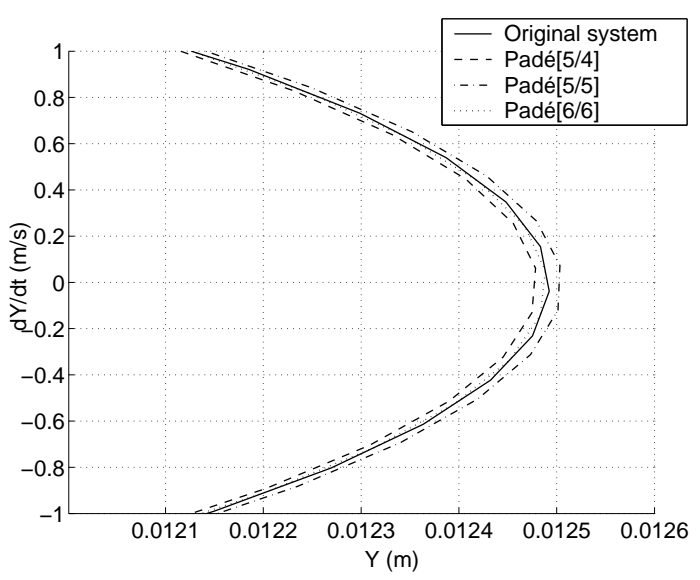

(b) Zoom

Figure 25: Limit cycles $(Y, \dot{Y})$ for $\mu=1.001 \mu_{0}$ by using the fractional approximants

\subsection{Estimate of the solution via the Harmonic Balance Method}

By using the two previous non-linear methods (the centre manifold approach and the fractional rational approximants), reduction and simplification of the mathematical complexity of the non-linear equations has been carried out. The non-linear system resulting from these procedures may be considered one of the most simple forms of the original non-linear system. Now we may investigate a classical non-linear method which requires an initial assumption about the form of the solution as a function of time. In this study, the classical approximation of the solution as a truncated Fourier series will be used. We use the harmonic balance method, called the Alternate Frequency/Time domain method (AFT method), to approximate the final solution of the reduced and simplified system as a periodic solution. This method and the global harmonic balance methods are very useful non-linear approaches to systematically obtain the non-linear behaviour of general non-linear vibration problems ([33], [151], [25], [129], [143], [110], [111], and [173]). The non-linear system in (167) can be described by a set of non-linear ordinary differential equations of the form

$$
\dot{\mathbf{v}_{\mathbf{c}}}=\mathbf{f}^{\mathrm{NL}}\left(\mathbf{v}_{\mathbf{c}}\right)
$$

where $\mathbf{v}_{\mathbf{c}}=\left\{\begin{array}{ll}v_{c 1} & v_{c 2}\end{array}\right\}^{T}$, and $\mathbf{f}^{\mathbf{N L}}$ is the vector of the SOD approximants in $\left(v_{c 1}, v_{c 2}\right)$. Then, the Alternate Frequency/Time domain method is based upon assumptions about a Fourier expansion for the non-linear response of the non-linear system (168). We assume that the vector solution $\mathbf{v}_{\mathbf{c}}=\left\{\begin{array}{ll}v_{c 1} & v_{c 2}\end{array}\right\}^{T}$ can be expanded as a truncated Fourier series:

$$
\mathbf{v}_{\mathbf{c}}(t)=\mathbf{V}_{0}+\sum_{j=1}^{H}\left(\mathbf{V}_{2 j-1} \cos (j \omega t)+\mathbf{V}_{2 j} \sin (j \omega t)\right)
$$

where $\omega=2 \pi / T$ and $T$ defines the period of the system. $\mathbf{V}_{\mathbf{0}}, \mathbf{V}_{\mathbf{2 j - 1}}$ and $\mathbf{V}_{\mathbf{2} \mathbf{j}}$ are the vectors of Fourier coefficients. Usually, harmonic components become less significant when $j$ increases, and hence we may ignore the harmonic components if $j$ is superior to $H$; in this case, the number of the harmonic coefficients $H$ are selected to retain only the significant harmonics.

\subsubsection{Computational estimate of the harmonic coefficients}

The coefficients for all the harmonics of (169) must be balanced in order to obtain the final solution of the non-linear system (168). The determination of the harmonic coefficients is found by using an iterative process. Substituting the truncated Fourier series expansion (168) into equation (168) yields a set of linear algebraic equations for the $k^{t h}$-iterative periodic solution for the Newton-Raphson method

$$
(\mathbf{A}-\mathbf{J}) \mathbf{V}^{k}+\mathbf{F}^{\mathbf{N L}}+(\mathbf{A}-\mathbf{J}) \Delta \mathbf{V}^{k}=\mathbf{0}
$$


where $\mathbf{V}^{k}$ defines the $k^{t h}$-incremental vector of the Fourier coefficients of $\mathbf{v}_{\mathbf{c}}$ :

$$
\mathbf{V}^{k}=\left\{\mathbf{V}_{0}^{k^{T}}, \cdots, \mathbf{V}_{2 j-1}^{k}{ }^{T}, \mathbf{V}_{2 j}^{k}{ }^{T}, \cdots, \mathbf{V}_{2 H}^{k}{ }^{T}\right\}^{T}
$$

$\mathbf{A}$ and $\mathbf{J}$ are the jacobian matrices associated with the linear and non-linear parts of (168). $\mathbf{F}^{\mathbf{N L}}$ defines the vector of the Fourier coefficients of the non-linear function $\mathbf{f}^{\mathbf{N L}}$. By considering the expression $\mathbf{f}^{\mathbf{N L}}=\left\{f_{1}^{N L} f_{2}^{N L}\right\}$, the matrices $\mathbf{J}$ and $\mathbf{A}$ are given by

$$
\begin{aligned}
& \mathbf{J}=(\Gamma \otimes \mathbf{I})\left[\begin{array}{lll}
\ddots & & \\
& {\left[\begin{array}{ll}
\frac{\partial f_{1}^{N L}}{\partial v_{c 1}} & \frac{\partial f_{1}^{N L}}{\partial v_{c 2}} \\
\frac{\partial f_{2}^{N L}}{\partial v_{c 1}} & \frac{\partial f_{2}^{N L}}{\partial v_{c 2}}
\end{array}\right]} & \\
& &
\end{array}\right]\left(\Gamma^{-1} \otimes \mathbf{I}\right)
\end{aligned}
$$

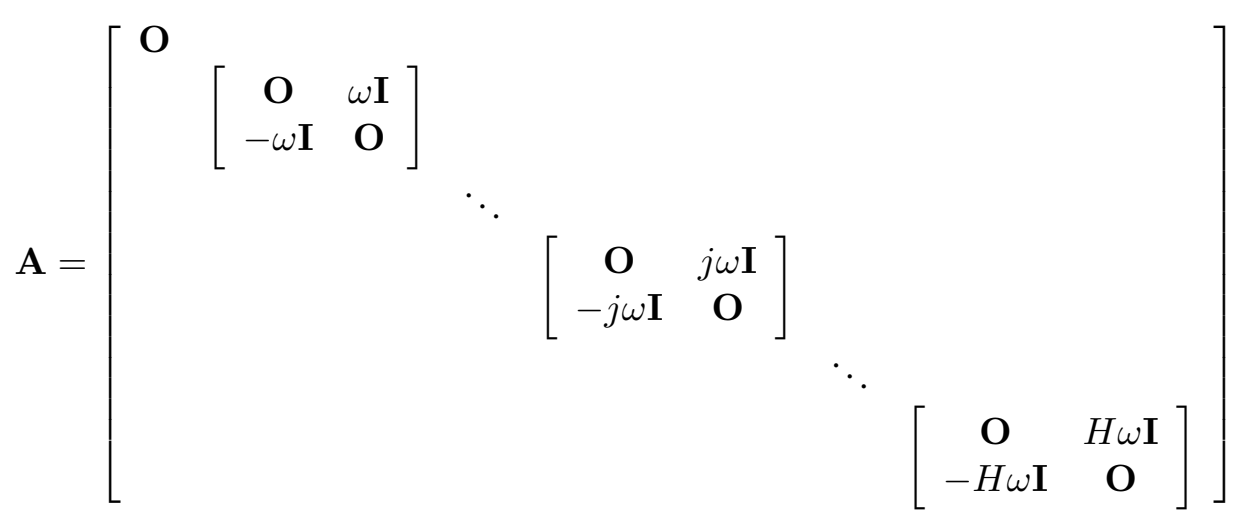

with the matrices $\mathbf{I}=\left[\begin{array}{ll}1 & 0 \\ 0 & 1\end{array}\right]$ and $\mathbf{O}=\left[\begin{array}{ll}0 & 0 \\ 0 & 0\end{array}\right]$ and $H$ represents the retained number of harmonic components for the solution's estimate.

The vectors $\Gamma$ and $\Gamma^{-1}$ make it possible to pass from the time domain to the frequency domain and vice versa. This procedure is called the Discret Fourier Transform ([25] and [129]) and the expression of $\Gamma$ and $\Gamma^{-1}$ are (with $q=2 H+1)$ :

$$
\begin{gathered}
{\left[\Gamma_{i j}\right]=\left\{\begin{array}{cl}
\frac{1}{q} & i=1 \quad \text { and } 1 \leq j \leq q \\
\frac{2}{q} \cos \left(\frac{(j-1) i \pi}{q}\right) & i=2,4, \cdots, q-1 \quad \text { and } \quad 1 \leq j \leq q \\
\frac{2}{q} \sin \left(\frac{(j-1)(i-1) \pi}{q}\right) & i=3,5, \cdots, q \quad \text { and } \quad 1 \leq j \leq q
\end{array}\right.} \\
{\left[\Gamma_{i j}\right]^{-1}=\left\{\begin{array}{cl}
\cos \left(\frac{(i-1) j \pi}{q}\right) & j=1 \quad \text { et } 1 \leq i \leq q \\
\sin \left(\frac{(i-1)(j-1) \pi}{q}\right) & j=3,5, \cdots, q \text { and } 1 \leq i \leq q
\end{array}\right.}
\end{gathered}
$$

Moreover, by considering the expression of the function $f_{i}$ defined in (168) as a SOD approximant $[M / N]_{f_{i}}$ :

$$
f_{i}\left(v_{c 1}, v_{c 2}\right)=\frac{\sum_{\alpha=0}^{M} \sum_{\beta=0}^{M} n_{i, \alpha \beta} v_{c 1}^{\alpha} v_{c 2}^{\beta}}{\sum_{\alpha=0}^{N} \sum_{\beta=0}^{N} d_{i, \alpha \beta} v_{c 1}^{\alpha} v_{c 2}^{\beta}}
$$


the expression of (172) may be easily obtained by calculating the expressions $\partial f_{i} / \partial v_{c 1}$ and $\partial f_{i} / \partial v_{c 2}$ :

$$
\begin{aligned}
\frac{\partial f_{i}}{\partial v_{c 1}}= & \frac{\alpha\left(\sum_{\alpha=0}^{M} \sum_{\beta=0}^{M} n_{i, \alpha \beta} v_{c 1}^{\alpha-1} v_{c 2}^{\beta} \times \sum_{\alpha=0}^{N} \sum_{\beta=0}^{N} d_{i, \alpha \beta} v_{c 1}^{\alpha} v_{c 2}^{\beta}-\sum_{\alpha=0}^{M} \sum_{\beta=0}^{M} n_{i, \alpha \beta} v_{c 1}^{\alpha} v_{c 2}^{\beta} \times \sum_{\alpha=0}^{N} \sum_{\beta=0}^{N} d_{i, \alpha \beta} v_{c 1}^{\alpha-1} v_{c 2}^{\beta}\right)}{\left(\sum_{\alpha=0}^{N} \sum_{\beta=0}^{N} d_{i, \alpha \beta} v_{c 1}^{\alpha} v_{c 2}^{\beta}\right)^{2}} \\
\frac{\partial f_{i}}{\partial v_{c 2}}= & \frac{\beta\left(\sum_{\alpha=0}^{M} \sum_{\beta=0}^{M} n_{i, \alpha \beta} v_{c 1}^{\alpha} v_{c 2}^{\beta-1} \times \sum_{\alpha=0}^{N} \sum_{\beta=0}^{N} d_{i, \alpha \beta} v_{c 1}^{\alpha} v_{c 2}^{\beta}-\sum_{\alpha=0}^{M} \sum_{\beta=0}^{M} n_{i, \alpha \beta} v_{c 1}^{\alpha} v_{c 2}^{\beta} \times \sum_{\alpha=0}^{N} \sum_{\beta=0}^{N} d_{i, \alpha \beta} v_{c 1}^{\alpha} v_{c 2}^{\beta-1}\right)}{\left(\sum_{\alpha=0}^{N} \sum_{\beta=0}^{N} d_{i, \alpha \beta} v_{c 1}^{\alpha} v_{c 2}^{\beta}\right)^{2}}
\end{aligned}
$$

The vector $\mathbf{F}^{\mathrm{NL}}$ that represents the vector of the Fourier coefficients of the non-linear function $\mathbf{f}^{\mathrm{NL}}$, is calculated by an iteration process ([25] and [129]), and by considering the Discret Fourier Transform DFT defined previously. Cameron and Griffin [25] indeed pointed out that the estimate of the vector $\mathbf{F}^{\mathrm{NL}}$ was often difficult to obtain from $\mathbf{V}^{k}=\left\{\mathbf{V}_{0}^{k^{T}}, \cdots, \mathbf{V}_{2 j-1}^{k}{ }^{T}, \mathbf{V}_{2 j}^{k^{T}}, \cdots, \mathbf{V}_{2 H}^{k}\right\}^{T}$ directly. Hence, they suggested that the vector $\mathbf{F}^{\mathrm{NL}}$ of the nonlinear function $\mathrm{f}^{\mathrm{NL}}$ be calculated by following the process:

$$
\mathbf{V} \quad-\frac{D F T^{-1}}{-}-\rightarrow \quad \mathbf{v}_{\mathbf{c}}(t) \quad \Longrightarrow \mathbf{f}_{\mathrm{NL}}(t) \quad--\frac{D F T}{-} \rightarrow \mathbf{F}^{\mathbf{N L}}
$$

Finally, the $(k+1)^{t h}$-iterative Fourier components estimate $\mathbf{V}^{k+1}$ is obtained from (170)

$$
\begin{gathered}
\boldsymbol{\Delta} \mathbf{V}^{k}=-(\mathbf{A}+\mathbf{J})^{-1}\left(\mathbf{F}^{\mathbf{N L}}+(\mathbf{A}+\mathbf{J}) \mathbf{V}^{k}\right) \\
\mathbf{V}^{k+1}=\mathbf{V}^{k}+\boldsymbol{\Delta} \mathbf{V}^{k}
\end{gathered}
$$

$\mathbf{V}^{k+1}$ is then used as a new estimate for the next iteration. The final solution of the system is obtained by considering the minimization of the error vector $\mathbf{R}$ and the associated convergences $\delta_{1}$ and $\delta_{2}$ :

$$
\begin{gathered}
\mathbf{R}=\mathbf{A} \mathbf{V}^{k}+\mathbf{F}^{\mathbf{N L}} \\
\delta_{1}=\sqrt{R_{0}^{2}+\sum_{j=1}^{H}\left(R_{2 j-1}^{2}+R_{2 j}^{2}\right)} \\
\delta_{2}=\sqrt{\Delta \mathbf{V}_{0}^{2}+\sum_{j=1}^{H}\left(\Delta \mathbf{V}_{2 j-1}^{2}+\Delta \mathbf{V}_{2 j}^{2}\right)}
\end{gathered}
$$

The complete scheme of the computational process is expressed in Figure 25. 


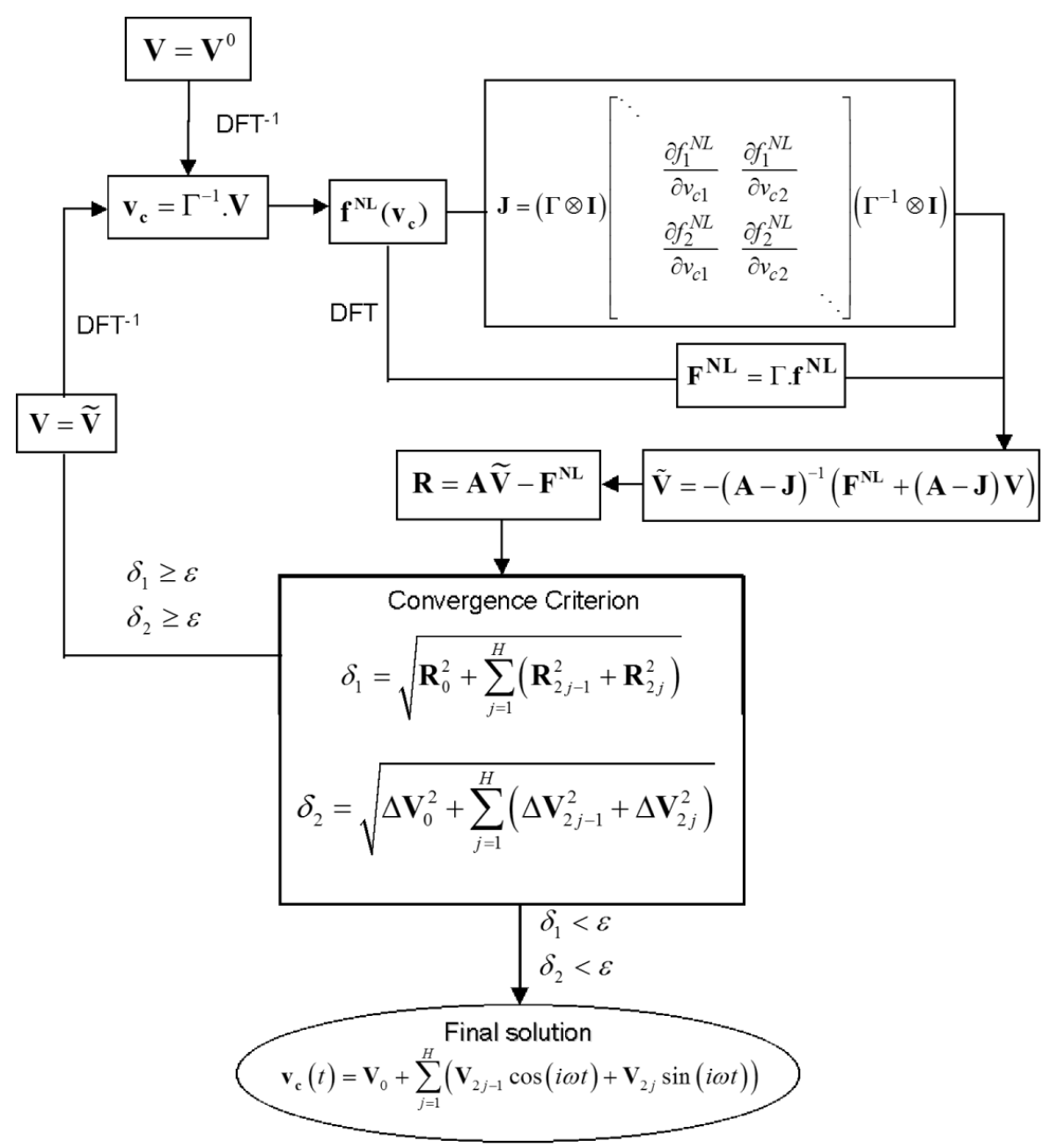

Figure 26: Computational process for the AFT method

\subsubsection{Numerical estimate of the system via the AFT method}

The numerical results in the time domain by using the Alternate Frequency/Time Domain method are shown in Figures 27 and 28 for various order $H$ of the harmonic coefficients. The results are compared with those obtained by using the SOD approximants solution that can be considered as the "exact" solution of the non-linear problem. The first order of the harmonic coefficients $(H=1)$ allows us to obtain a good estimate of the limit cycles. Moreover, the second or higher order of the harmonic coefficients $(H \geq 2)$ enable us to obtain the same limit cycles as those obtained by the Padé SOD approximants. The values of all the harmonic coefficients for one, two and three harmonics are given in Table 1. The associated evolutions of these harmonic coefficients during the iteration process of the Alternate Frequency/Time Domain method are given for the variable $v_{c 1}$ and $v_{c 2}$ in Figures 29-30, and Figures 31-32 respectively. It appears that the calculation of Fourier coefficients (and the associated limit cycles) requires only a small number of iterations. Therefore, it may be noted that the value of the Fourier coefficients are complex, since they are defined in the centre manifold space. So, the limit cycles of the non-linear system are obtained by using the reverse transformation in order to go from the centre manifold space with complex variables to the physical space with real variables.

Finally, one of the practical computational problems for wide application of methods based on the balance of harmonics, such as the Alternate frequency/Time Domain method, is believed to be the determination of a good initial estimate for the iteration process. We may indeed observe that the Newton-Raphson approach may fail to converge with the solution in the AFT method if the initial estimate of the solution is not sufficiently accurate [25]. In many applications, a perturbation approach is suggested to avoid this problem when the Newton-Raphson method fails [151]. 


\begin{tabular}{|c||c||c||c|}
\hline Fourier components & $H=1$ & $H=2$ & cas $H=3$ \\
\hline \hline$V_{1,0}$ & $-0.685-0.0102 \mathrm{i}$ & $-0.686-0.0102 \mathrm{i}$ & $-0.685-0.0102 \mathrm{i}$ \\
\hline$V_{2,0}$ & $-0.685+0.0102 \mathrm{i}$ & $-0.686+0.0102 \mathrm{i}$ & $-0.685+0.0102 \mathrm{i}$ \\
\hline$V_{1,1}$ & $1.7453+1.0786 \mathrm{i}$ & $1.7453+1.0812 \mathrm{i}$ & $1.7458+1.0808 \mathrm{i}$ \\
\hline$V_{2,1}$ & $1.7453-1.0786 \mathrm{i}$ & $1.7453-1.0812 \mathrm{i}$ & $1.7458-1.0808 \mathrm{i}$ \\
\hline$V_{1,2}$ & $-1.0775+1.7377 \mathrm{i}$ & $-1.0805+1.739 \mathrm{i}$ & $-1.0801+1.7385 \mathrm{i}$ \\
\hline$V_{2,2}$ & $-1.0775-1.7377 \mathrm{i}$ & $-1.0805-1.739 \mathrm{i}$ & $-1.0801-1.7385 \mathrm{i}$ \\
\hline$V_{1,3}$ & 0 & $0.0171+0.0215 \mathrm{i}$ & $0.0166+0.0212 \mathrm{i}$ \\
\hline$V_{2,3}$ & 0 & $0.0171-0.0215 \mathrm{i}$ & $0.0166-0.0212 \mathrm{i}$ \\
\hline$V_{1,4}$ & 0 & $-0.0147+0.0388 \mathrm{i}$ & $-0.0144+0.0382 \mathrm{i}$ \\
\hline$V_{2,4}$ & 0 & $-0.0147-0.0388 \mathrm{i}$ & $-0.0144-0.0382 \mathrm{i}$ \\
\hline$V_{1,5}$ & 0 & 0 & $0.0002+0.0007 \mathrm{i}$ \\
\hline$V_{2,5}$ & 0 & 0 & $0.0002-0.0007 \mathrm{i}$ \\
\hline$V_{1,6}$ & 0 & 0 & $-0.0002+0.0008 \mathrm{i}$ \\
\hline$V_{2,6}$ & 0 & 0 & $-0.0002+0.0008 \mathrm{i}$ \\
\hline
\end{tabular}

Table 1: Fourier components for various order $H$ of the periodic solution

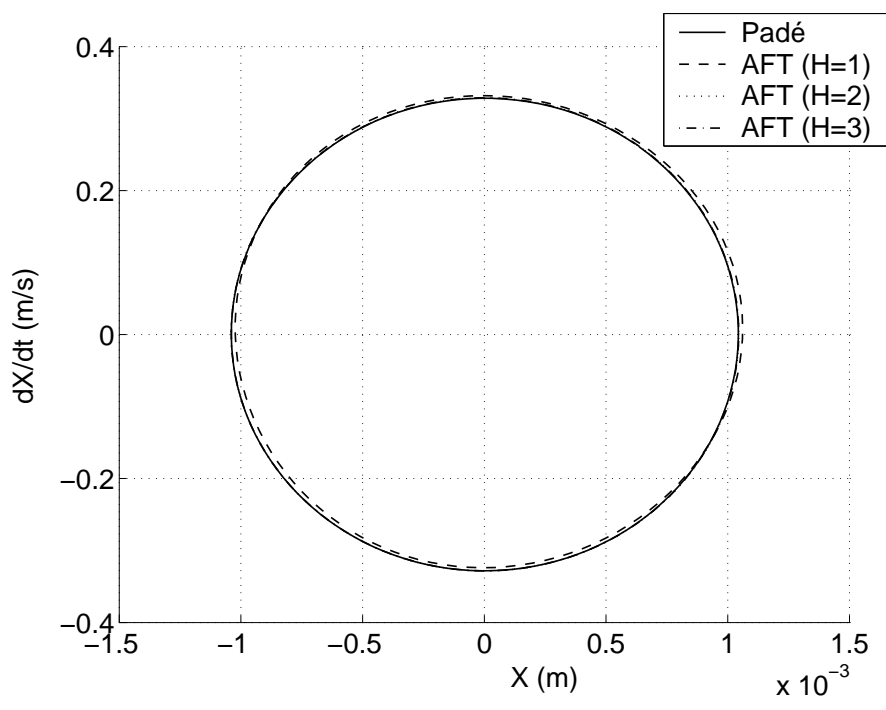

(a) Limit $\operatorname{cycles}(X, \dot{X})$

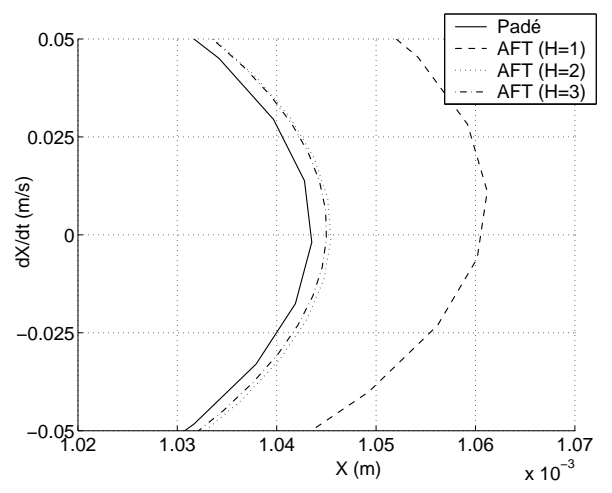

(b) Zoom

Figure 27: $\operatorname{Limit} \operatorname{cycles}(X, \dot{X})$ for $\bar{\mu}=1.001 \mu$ by using the AFT method 


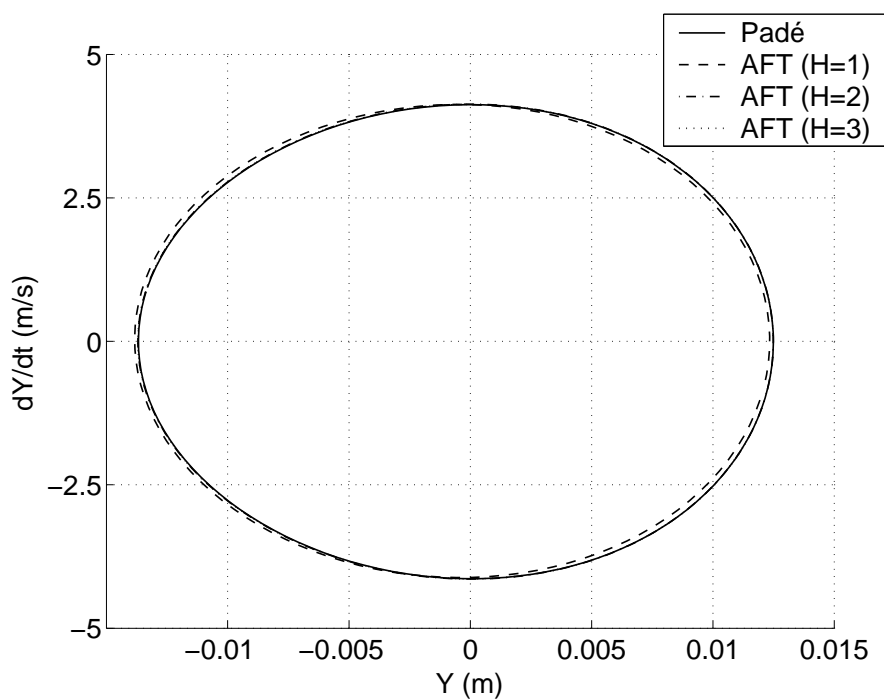

(a) Limit cycles $(Y, \dot{Y})$

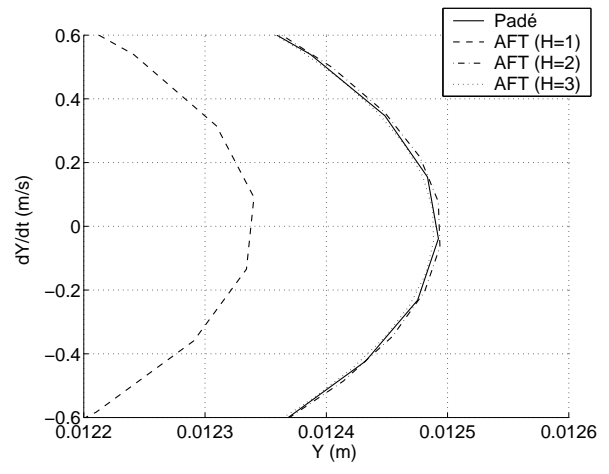

(b) Zoom

Figure 28: Limit cycles $(Y, \dot{Y})$ for $\bar{\mu}=1.001 \mu$ by using the AFT method

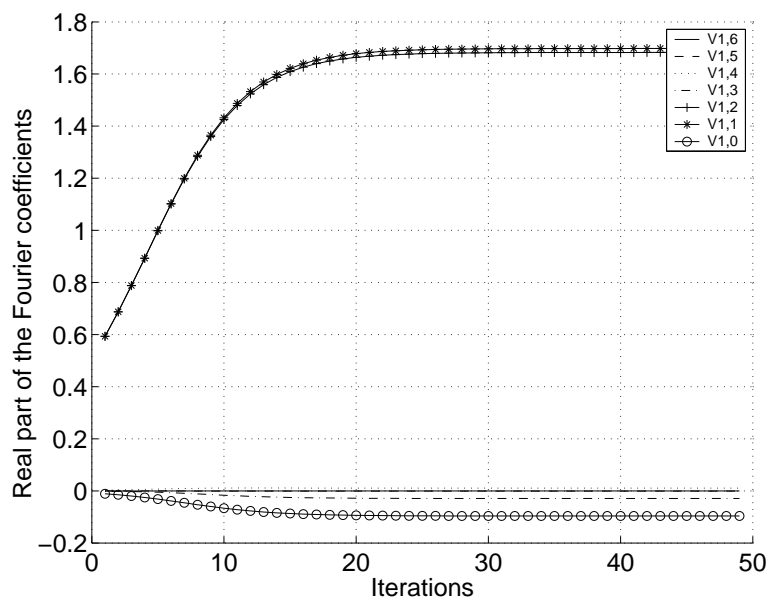

(a) Evolution of the real part

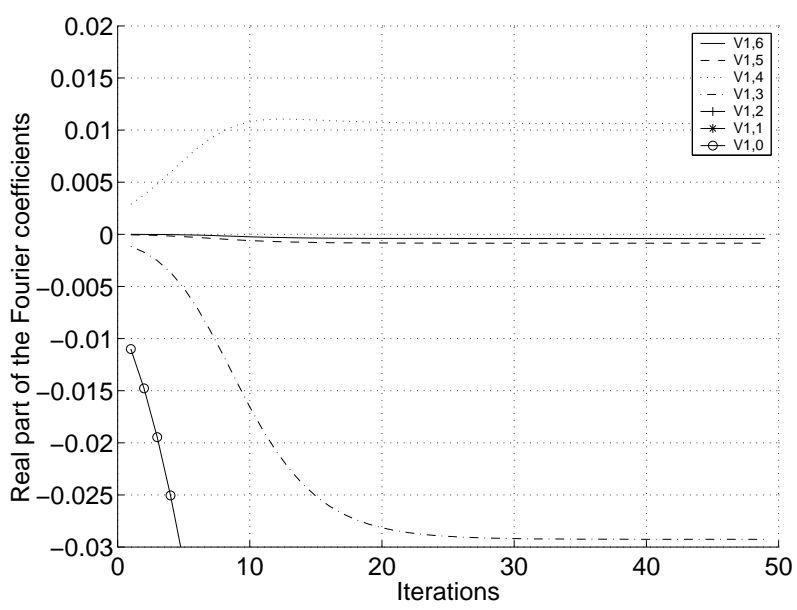

(b) Zoom

Figure 29: Evolution of the real part of the Fourier components for $v_{c 1}$ 


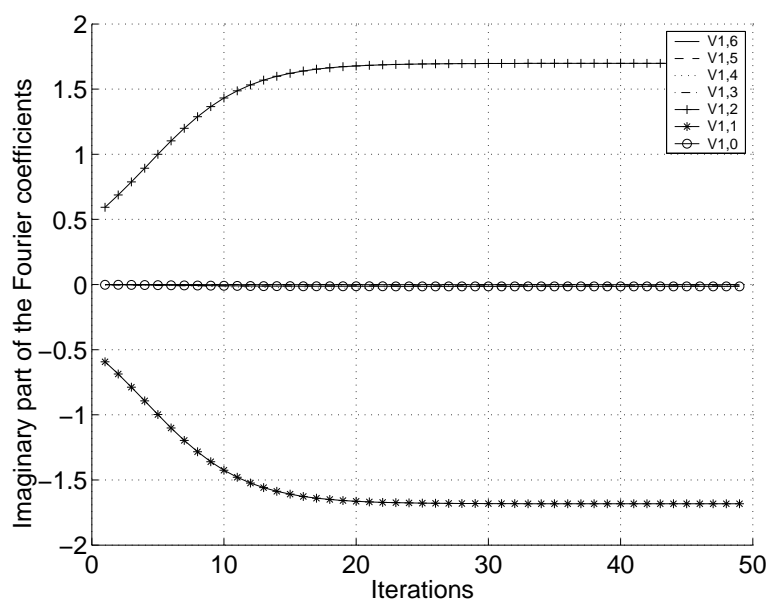

(a) Evolution of the imaginary part

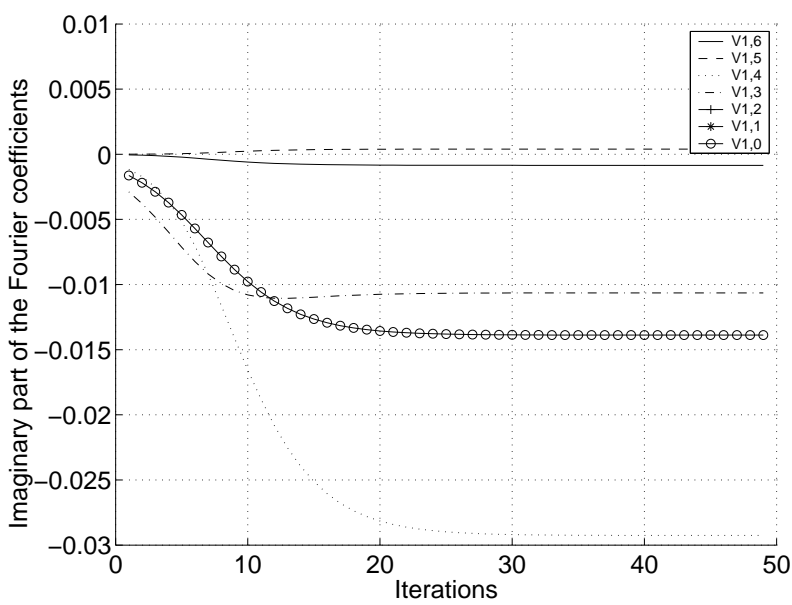

(b) Zoom

Figure 30: Evolution of the imaginary part of the Fourier components for $v_{c 1}$

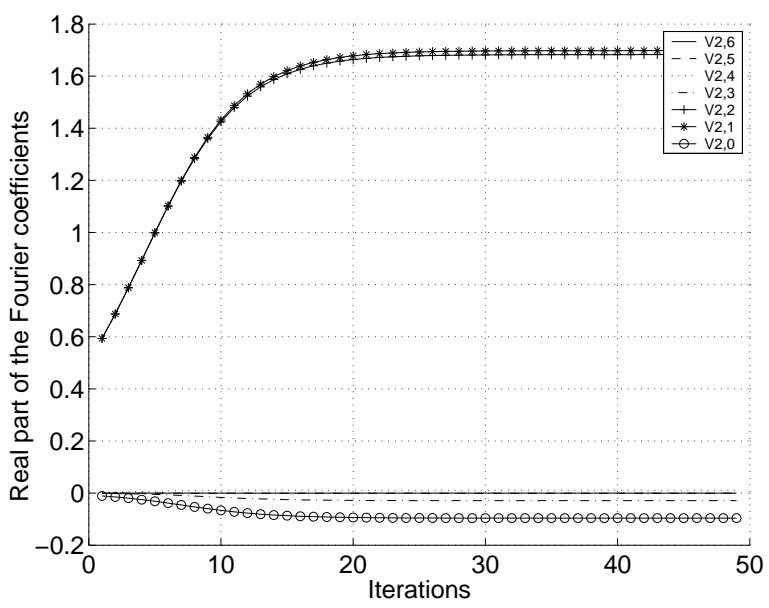

(a) Evolution of the real part

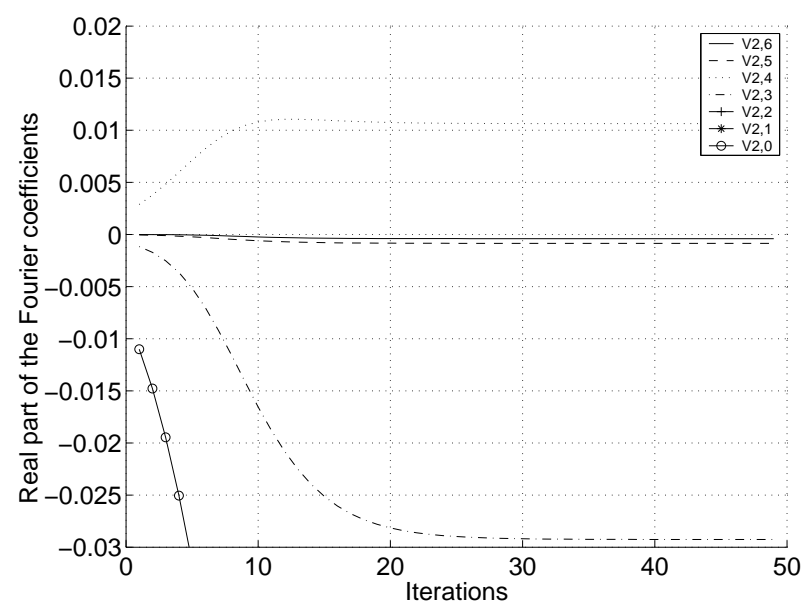

(b) Zoom

Figure 31: Evolution of the real part of the Fourier components for $v_{c 2}$

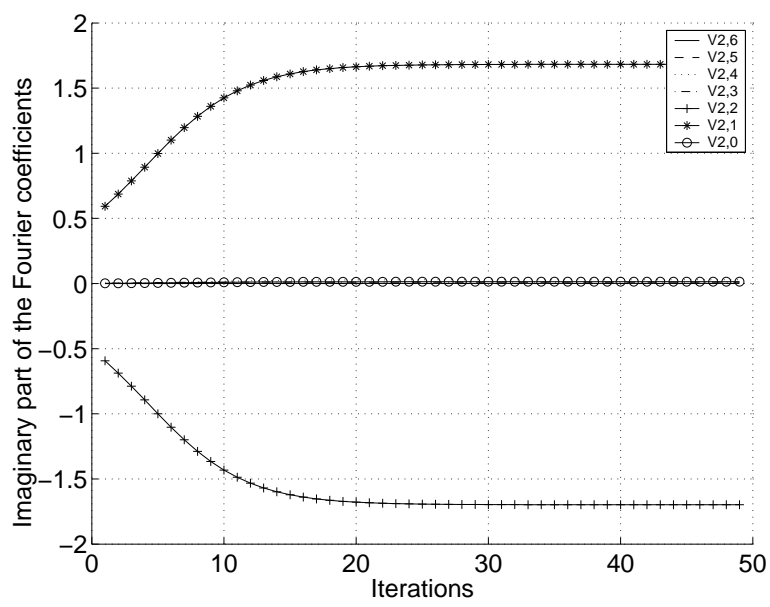

(a) Evolution of the imaginary part

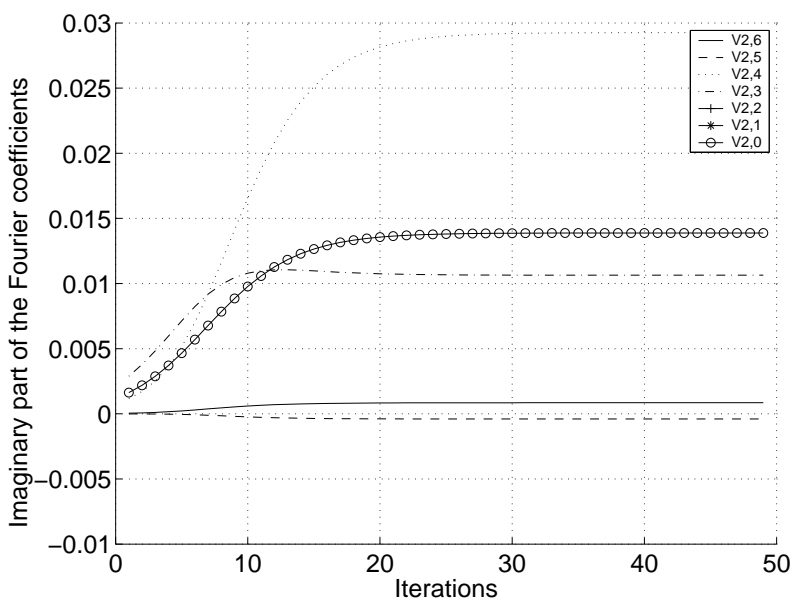

(b) Zoom

Figure 32: Evolution of the imaginary part of the Fourier components for $v_{c 2}$ 


\subsection{Advantages of applying non-linear methods}

The purpose of this section is to demonstrate the advantages of the non-linear methods and more particularly the fractional approximants. First, comparisons of the CPU times for the classical $4^{\text {th }}$-order Runge-Kutta procedure and each non-linear method are investigated. Second, some considerations about the advantage of the fractional approximants are described; the possible convergence of the fractional approximants outside the domain of convergence of the series they approximate is investigated. Finally, we will show that one of the most important aspects of applying the fractional approximants after the centre manifold approach lies in the fact that they require fewer terms than the associated Taylor series (obtained by using the centre manifold approach of $k^{\text {th }}$-order) in order to obtain an accurate estimate of the non-linear solution of the problem, and that it may be possible to obtain a good approximation of the solution even if the associated centre manifold estimate of $k^{t h}$-order diverges or is not sufficient to approximate the non-linear system.

\subsubsection{Computational Time}

Using the non-linear investigation and a classical integration scheme ( $4^{\text {th }}$-order Runge-kutta procedure) an estimate and comparison of the methods' CPU time may be investigated. The computational calculations need about 2000 CPU seconds by using the classical $4^{\text {th }}$-order Runge-Kutta procedure. By applying the centre manifold approach $\left(3^{\text {rd }}\right.$-order for example) and the fractional SOD approximants $[5 / 4]_{f}$, these calculations only need about $200 \mathrm{CPU}$ time and 50 CPU seconds. All the CPU times for various orders of the centre manifold approach and various numerators and denominators of the SOD approximants are given in Table 2. The centre manifold and the fractional approximants make it possible to save time by reducing the number of degree-of-freedom and by simplifying the number of nonlinear terms of the reduced centre manifold system, respectively. Moreover, the use of the Alternate frequency/Time Domain method saves time by assuming a Fourier series expansion for the periodic solution of the final non-linear reduced and simplified system. In conclusion, the amount of CPU-time when applying the non-linear methods is significantly less than that of the classical Runge-Kutta 4 procedure. Therefore, these methods provide goods results for our problem by reducing and simplifying the original system without losing all the non-linear behaviour of the problem. It may be observed that the more complex the original non-linear system is, with many degrees-of-freedom, the more interesting the centre manifold approach is, allowing us to save time and to determine rapidly and efficiently the limit cycle amplitudes.

\begin{tabular}{|c||c|}
\hline Numerical methods & CPU time $(\mathrm{sec})$ \\
\hline \hline Original system - Runge-Kutta of $4^{t h}$-order & 2000 \\
\hline Center manifold approach $\left(3^{t h}\right.$-order) & 200 \\
\hline Center manifold approach $\left(4^{t h}\right.$-order $)$ & 500 \\
\hline Center manifold approach $\left(5^{t h}\right.$-order) & 1200 \\
\hline Symmetric-Off-Diagonal Approximants [5/4] & 50 \\
\hline Symmetric-Off-Diagonal Approximants [5/5] & 135 \\
\hline Symmetric-Off-Diagonal Approximants [6/6] & 280 \\
\hline Alternate Frequency/Time Domain method $(H=1)$ & 13 \\
\hline Alternate Frequency/Time Domain method $(H=2)$ & 30 \\
\hline Alternate Frequency/Time Domain method $(H=3)$ & 45 \\
\hline
\end{tabular}

Table 2: CPU time for various numerical non-linear estimates

\subsubsection{Extension of the domain of convergence via the fractional approximants}

One of the well-known advantages of the rational approximants is their possible convergence outside the domain of convergence of the series they approximate. Due to the $k^{t h}$-order polynomial approximation $\mathbf{h}$ of the stable variables $\mathbf{v}_{\mathbf{s}}$ as a power series in centre variables $\mathbf{v}_{\mathbf{c}}$, the reduced system defined in equation (146) has a circle of convergence $R$ (with $R \leq R_{\infty}$ where $R_{\infty}$ is the circle of convergence for the power series associated with the polynomial approximation $\mathbf{h}$ when $k \rightarrow+\infty$ ). If the initial conditions are taken outside this circle of convergence $R$, the series defined 
in equation (146) diverge. We now examine the fractional rational approximants associated with the power series obtained via the centre manifold approach that has a circle of convergence $R$. By considering the same initial conditions, it may be observed that the fractional approximants converge and produce the same non-linear solution as the original non-linear system, as illustrated in Figures 33 and 34. In this case, the sequence of rational approximants converge outside the circle of convergence $R$; the fractional approximants enhance the convergence of the series expansions of the centre manifold theory. In this section, it is important to understand that the polynomial approximation is assumed to be sufficient in order to obtain a good approximation of the non-linear behaviour of the reduced system if the initial conditions are taken inside the circle of convergence $R$.

To examine the capability and suitability of this last property and more generally of the rational approximants, we use various initial conditions taken inside or outside the circle of convergence $R$ (first case: $V_{1, k}^{0}=V_{2, k}^{0}=20$; second case: $V_{1, k}^{0}=V_{2, k}^{0}=10+10 i$; third case: $V_{1, k}^{0}=V_{2, k}^{0}=-30 i$; fourth case: $V_{1, k}^{0}=V_{2, k}^{0}=2+2 i$; fifth case: $V_{1, k}^{0}=V_{2, k}^{0}=1$ for $\left.k=0,1, \cdots, 4\right)$. As indicated in Figures 35 and 36, The Fourier coefficients and the associated non-linear responses are correctly estimated in all cases. Then, the extension of the domain of convergence via the fractional rational approximants becomes interesting, even if the value of the new circle of convergence due to the fractional approximants is an unknown parameter.

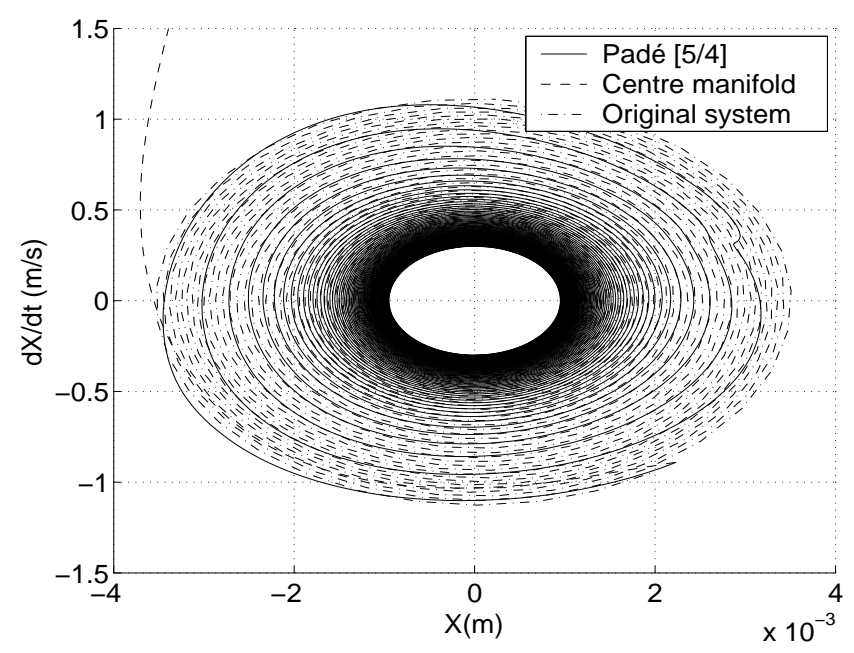

Figure 33: Evolution of the limit cycles $(X, \dot{X})$ by using the Runge-Kutta 4, the center manifold approach and the fractional approximants

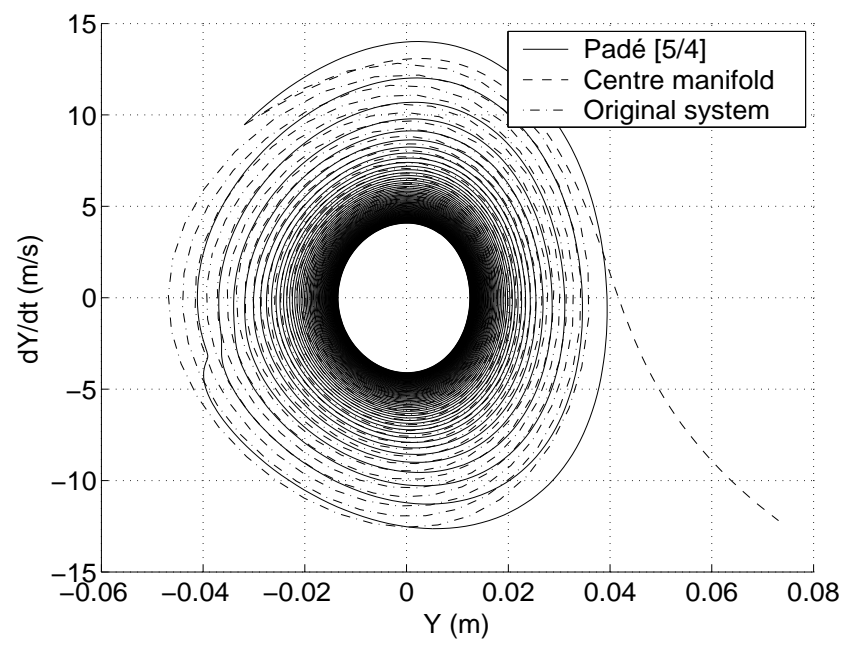

Figure 34: Evolution of the limit cycles $(Y, \dot{Y})$ by using the Runge-Kutta 4, the center manifold approach and the fractional approximants 


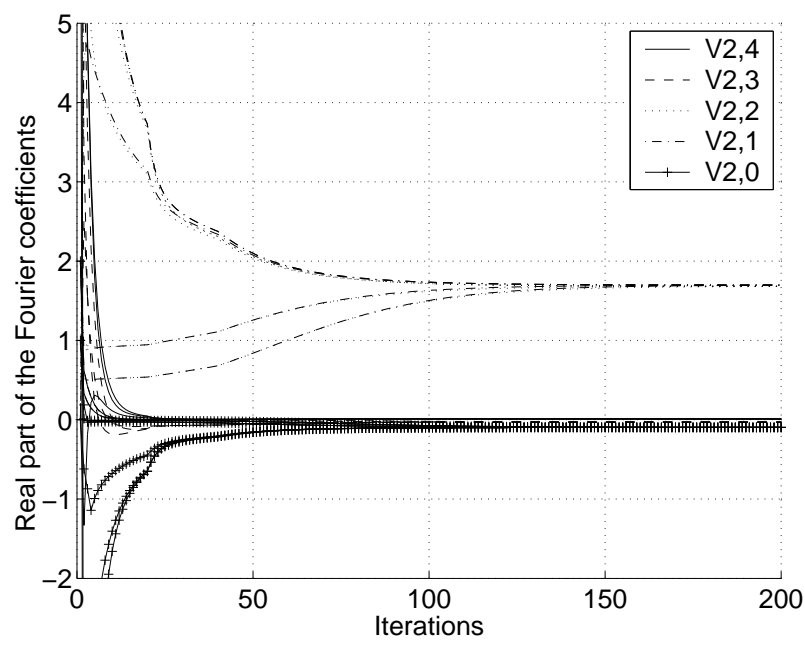

(a) Evolution of the real part

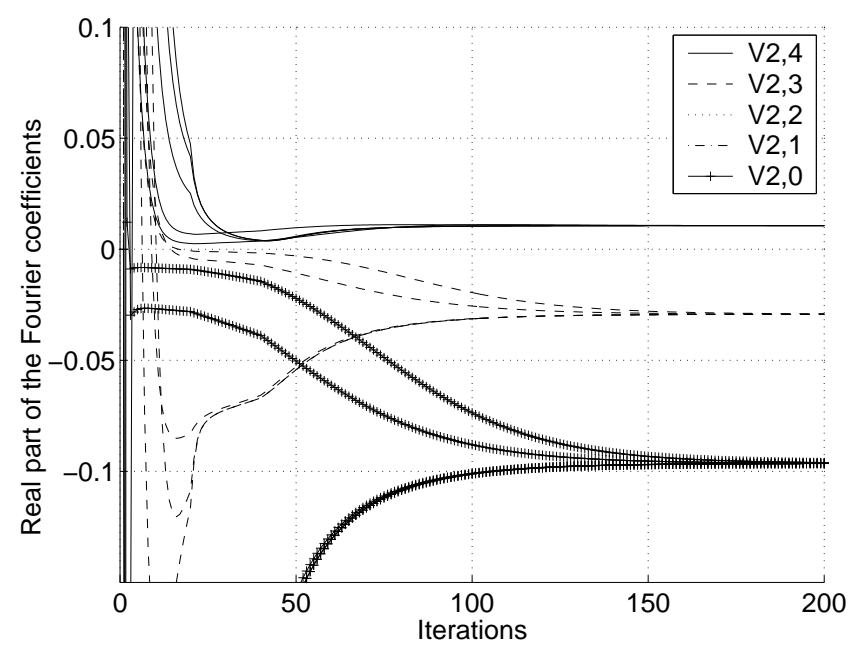

(b) Zoom

Figure 35: Evolution of the real part of the Fourier coefficients for $v_{c 2}$

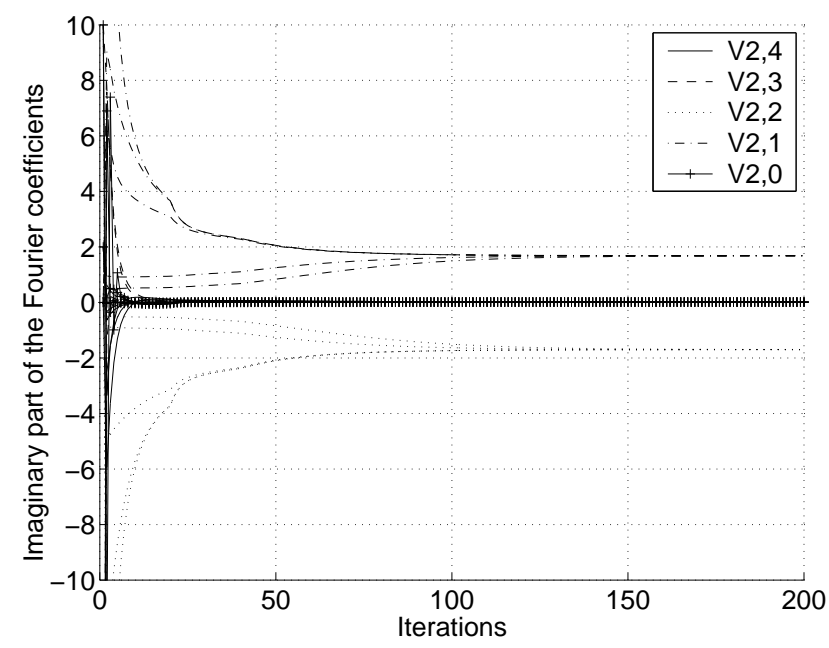

(a) Evolution of the imaginary part

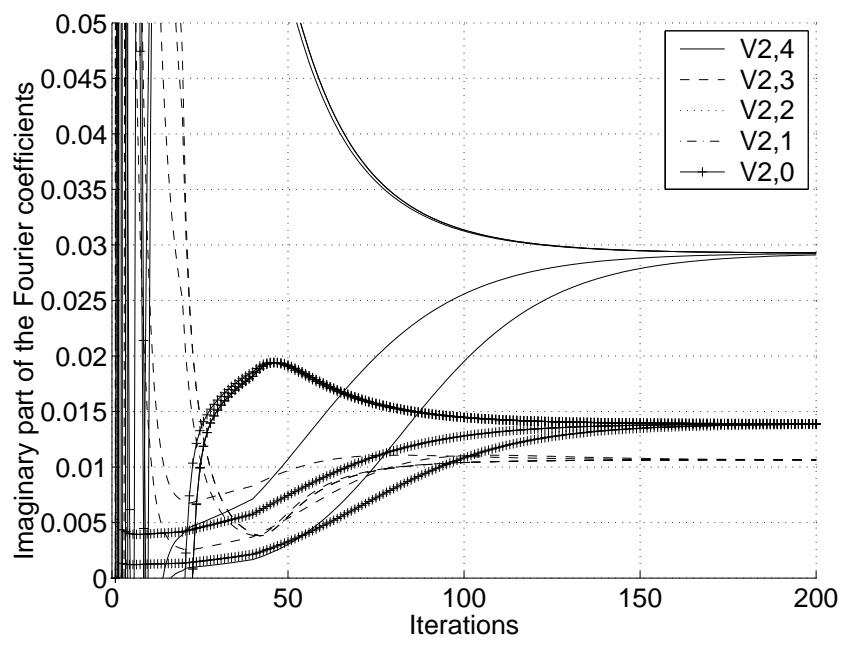

(b) Zoom

Figure 36: Evolution of the imaginary part of the Fourier coefficients for $v_{c 2}$

\subsubsection{Extension of the center manifold via the fractional approximants}

The interest of these rational approximants is that they require fewer terms than the associated Taylor series in order to obtain an accurate approximation of the behaviour of the complete non-linear system. In any case, the rational approximation has a greater range of validity than the polynomial one and make it possible to obtain an approximation of the solution even if the associated centre manifold approximation diverges or is not sufficient to approximate the non-linear solution near the equilibrium point. To demonstrate this advantage of the rational approximants, the previous system is applied by changing only the value of the parameter $C_{2}=300 \mathrm{~N} / \mathrm{m} / \mathrm{sec}$. In this case the Hopf bifurcation point is obtained for $\mu_{0}=0.28$. The displacements $X$ and $Y$ and velocities $\dot{X}$ and $\dot{Y}$ obtained by using a classical integration Runge-Kutta 4 near the Hopf bifurcation point $\mu=1.01 \mu_{0}$ are given in Figures 37 and 38, respectively. The associated limit cycles $(X, \dot{X})$ and $(Y, \dot{Y})$ are given in Figures 39. It may be noted that the non-linear oscillation of the displacements and velocities grow more rapidly. 


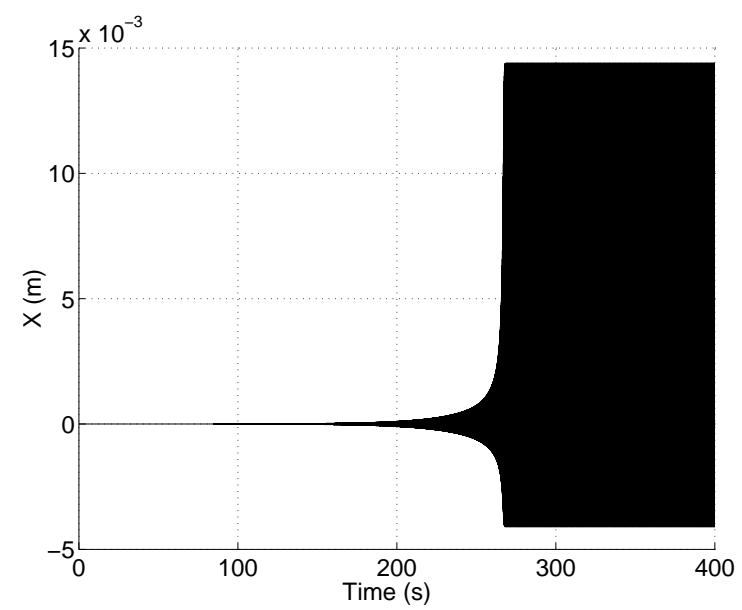

(a) Displacement $X(t)$

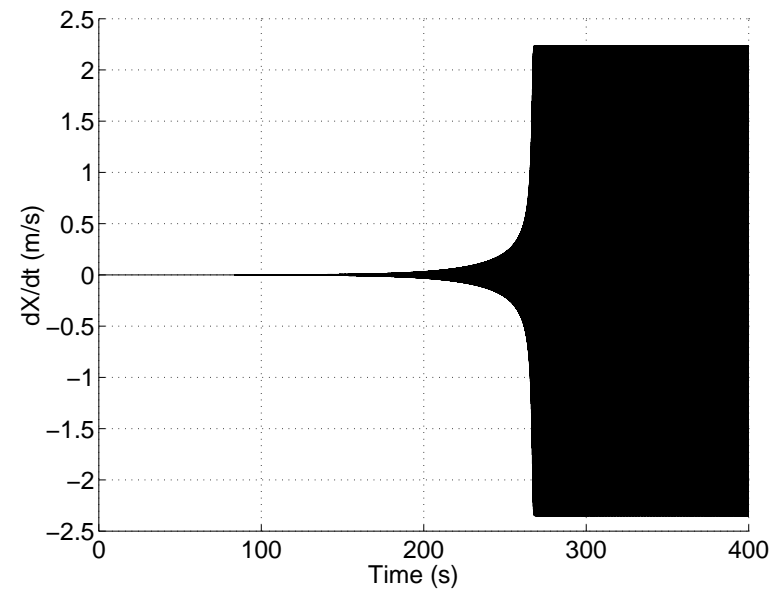

(b) Velocity $\dot{X}(t)$

Figure 37: Displacement $X(t)$ and velocity $\dot{X}(t)$ for $\mu=1.01 \mu_{0}$

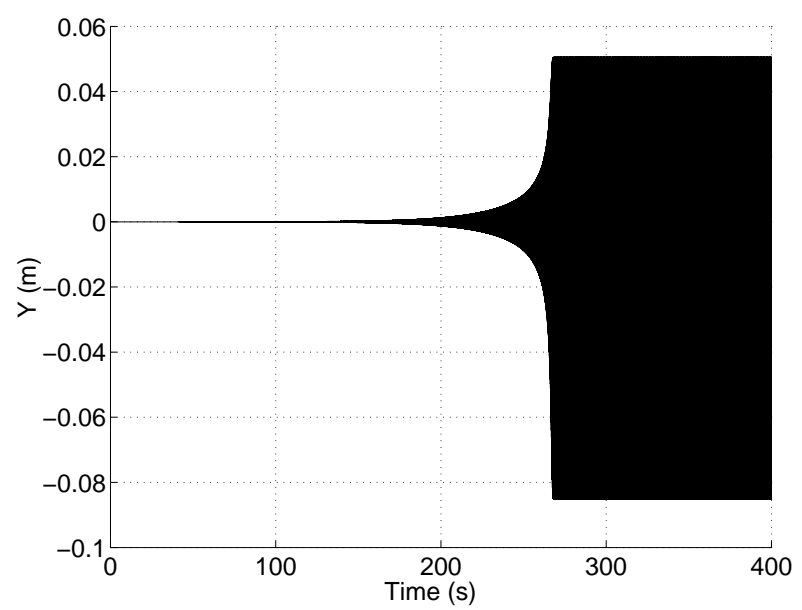

(a) Displacement $Y(t)$

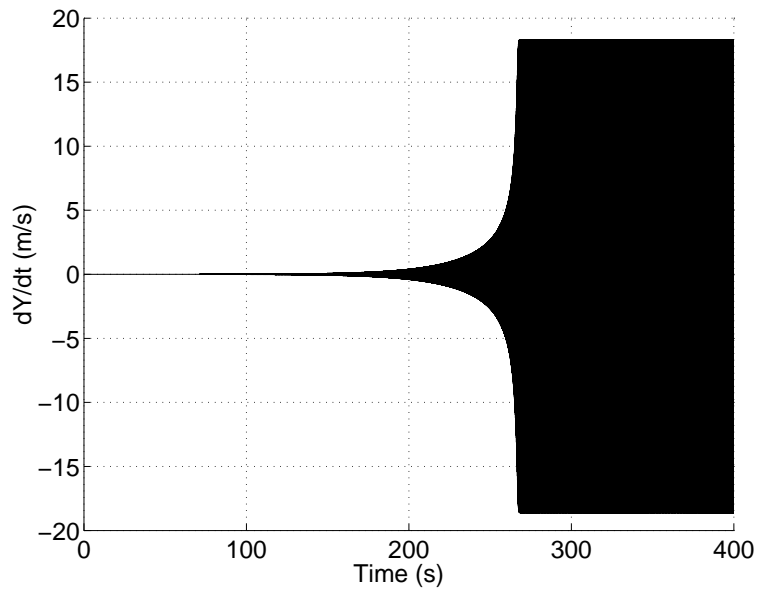

(b) Velocity $\dot{Y}(t)$

Figure 38: Displacement $Y(t)$ and velocity $\dot{Y}(t)$ for $\mu=1.01 \mu_{0}$

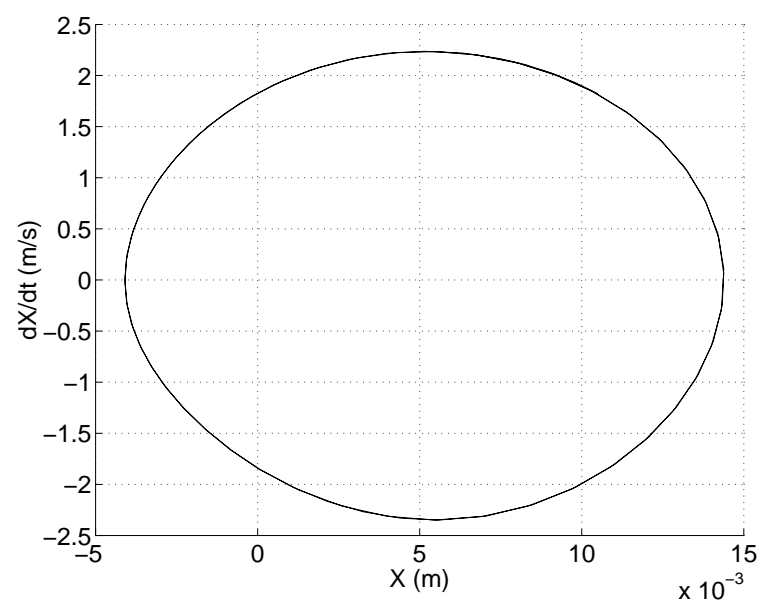

(a) Limit cycle $(X, \dot{X})$

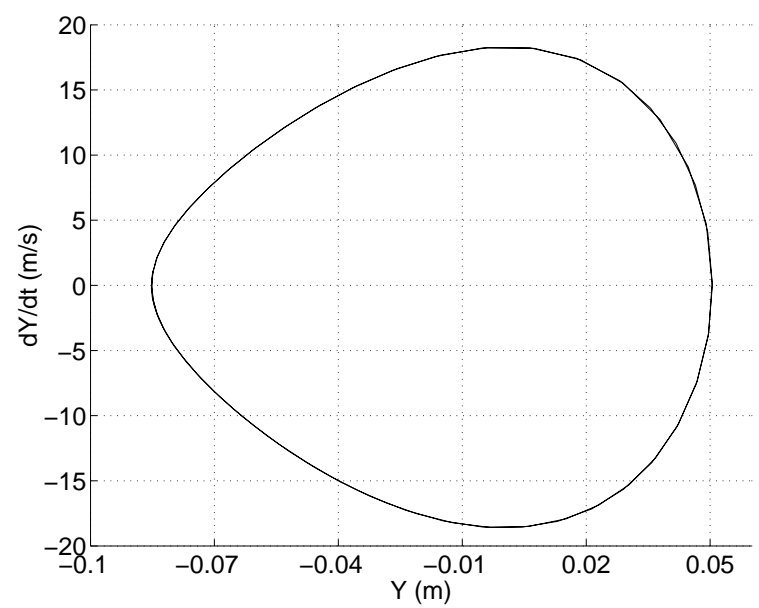

(b) Limit cycle $(Y, \dot{Y})$

Figure 39: Limit cycles $(X, \dot{X})$ and $(Y, \dot{Y})$ for $\mu=1.01 \mu_{0}$ 
By applying the centre manifold theory in this case, we observe that the limit cycles for $\mu=1.01 \mu_{0}$ obtained with various approximations of the stable variables as a power series in the centre variables of order 2, 3, 4 or 5 diverge. This implies that the polynomial approximation is not sufficient to obtain a good estimate of the stable variable contributions and of the non-linear behaviour of the dynamical system. However, we apply the symmetric-off-diagonal rational approximants in order to extend the non-linear expression of the centre manifold system of order 5 (we know that the associated limit cycles diverge) and to estimate the associated limit cycles: the purpose of using the rational fractional approximants is to obtain an approximate non-linear response of the complete system by considering that the approximants require fewer terms than the associated Taylor series (defined by the centre manifold of order 5 in this case). In fact, the rational approximants also allow us to simplify the non-linear terms of the centre manifold of order 5. By using the approximants $[8 / 7]_{f}$ and the Alternate/Frequency Time domain method (with $H=3$ ), we observe that the Fourier coefficients of each variable $v_{c 1}$ and $v_{c 2}$ converge, as illustrated in Figures 41 and 42 , respectively. By comparing the limit cycles obtained by considering these Fourier coefficients with those obtained via the previous integration of the full original system, we observe that the limit cycles are acceptable, as illustrated in Figure 42 and Figure 43. The harmonic balance method is used in this case only to accelerate obtaining the final solution by assuming the form of the response as a truncated Fourier series. The domain of validity for the centre manifold approach is extended only due to the application and properties of the rational fractional approximants.

So, in this case, the rational fractional approximants allow us to enhance the convergence of the series expansions of the centre manifold theory. Moreover, the sequence of rational fractional approximants converge even if the associated series does not; we can than extend our domain of convergence and good agreements are found between the original and reduced system. Moreover, this extension of the centre manifold via the fractional rational approximants requires less computer resources: the use of the rational approximants allows us to consider lower order approximation of the polynomial approximation $\mathbf{h}$ (with $\mathbf{v}_{\mathbf{s}}=\mathbf{h}\left(\mathbf{v}_{\mathbf{c}}\right)$ ). The CPU times associated with all the simulations are given in Table 3.

Obtaining the centre manifold coefficients $a_{k, i j}$ associated which the stable variables $\mathbf{v}_{\mathbf{s}}$ (with $\mathbf{v}_{\mathbf{s}}=\sum_{p=i+j=2}^{q} \sum_{j=0}^{p} \mathbf{a}_{\mathbf{i j}} v_{c 1}^{i} v_{c 2}^{j}$ and $q \geq 2$ ) may pose particularly serious difficulties. This is why the sole use of the centre manifold approach is not very convenient, requiring a great deal of labour, especially for the computational calculation of the coefficients $a_{k, i j}$ defined previously.

There are two important points to make here. First, this procedure used 316 non-linear terms in order to obtain an estimation of the limit cycle amplitude, as indicated in Table 3; in the case of the centre manifold approach, 512 non-linear terms are not sufficient to obtain the limit cycle amplitudes. So, we extend the domain of validity of the problem and simplify the non-linear terms. Second, we obtain good agreement with the complete non-linear system. This procedure makes it possible to reduce the number of degree-of-freedom of the original non-linear system and to simplify the non-linear terms. The great advantage of the use of rational approximants after the centre manifold method in comparison with the normal form approach defined in Section 2.3 is clearly demonstrated by considering this extension of the centre manifold approach. Determining the normal form is usually obtained by considering the power series defined by the centre manifold approach; due to the fact that the normal form is another power series containing only the relevant non-linear terms, it may be assumed that if the power series obtained by applying the centre manifold diverge, the associated simplification via the normal form also diverges. This property can be understood by considering the procedure of the normal form transformation defined in Section 2.3.

\begin{tabular}{|c||c||c||c|}
\hline Methods & CPU time (s) & Degree-of-freedom & Non-linear terms \\
\hline \hline Original system - Runge-Kutta of $4^{t h}$-order & 2000 & 4 & 96 \\
\hline Center manifold approach $q^{t h}$-order with $q \leq 5$ & diverge & 2 & 512 \\
\hline Extension via the Padé [8/7] & 300 & 2 & 316 \\
\hline Padé [8/7] + AFT $(H=3)$ & 50 & 2 & 316 \\
\hline
\end{tabular}

Table 3: Advantages of the extension of the center manifold approach via the fractional approximants 


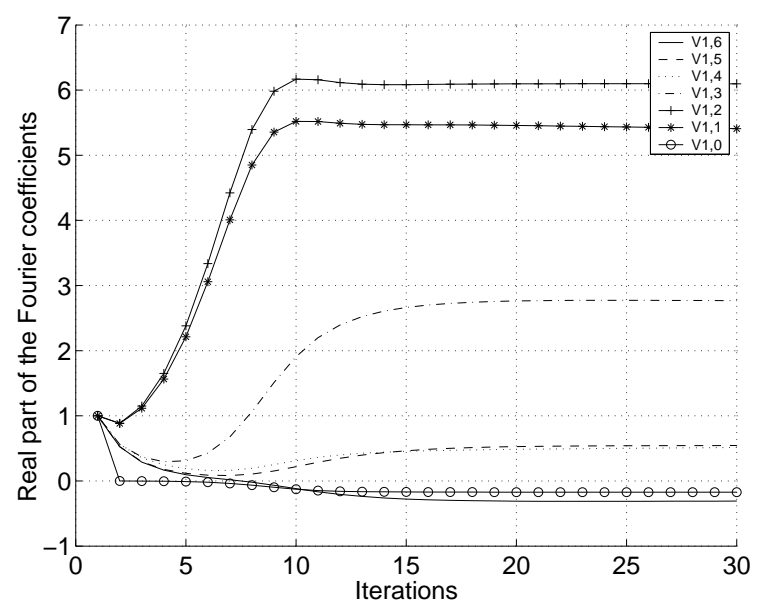

(a) Evolution of the real part

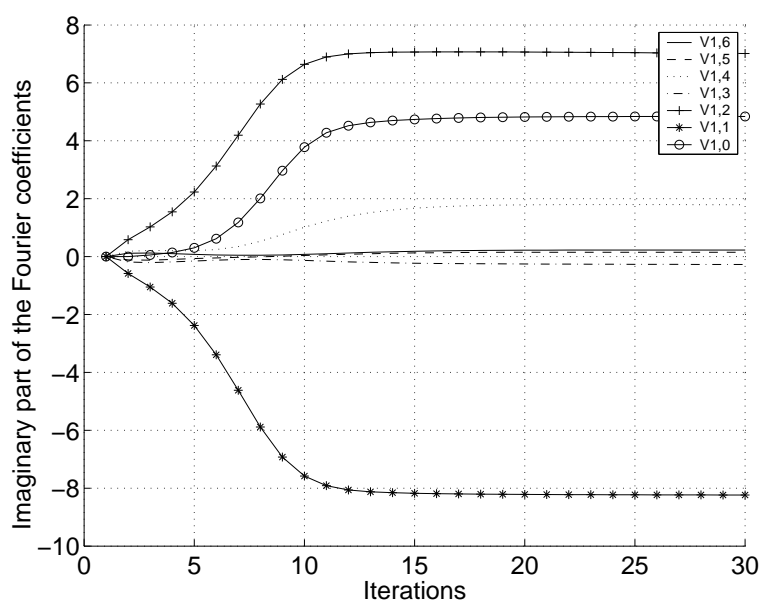

(b) Evolution of the imaginary part

Figure 40: Evolution of the Fourier coefficients for $v_{c 1}$

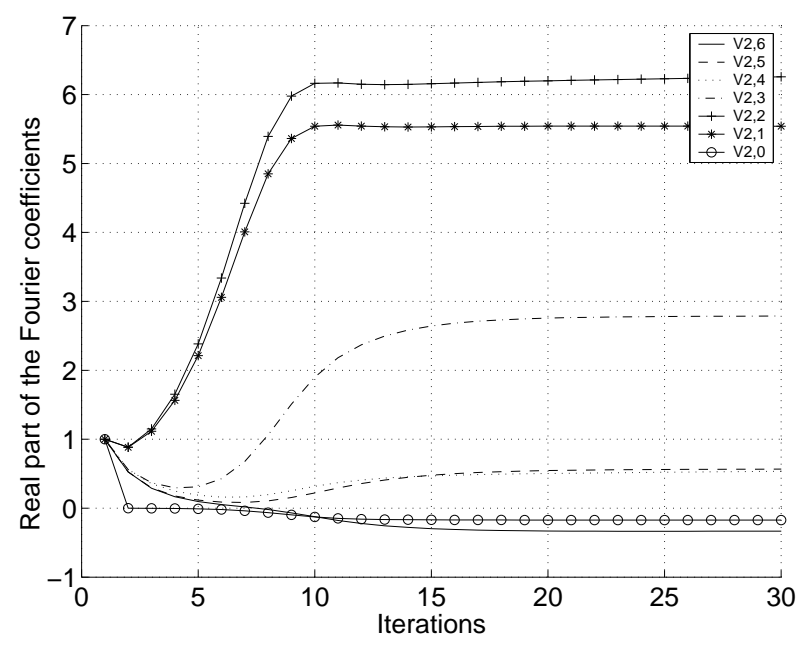

(a) Evolution of the real part

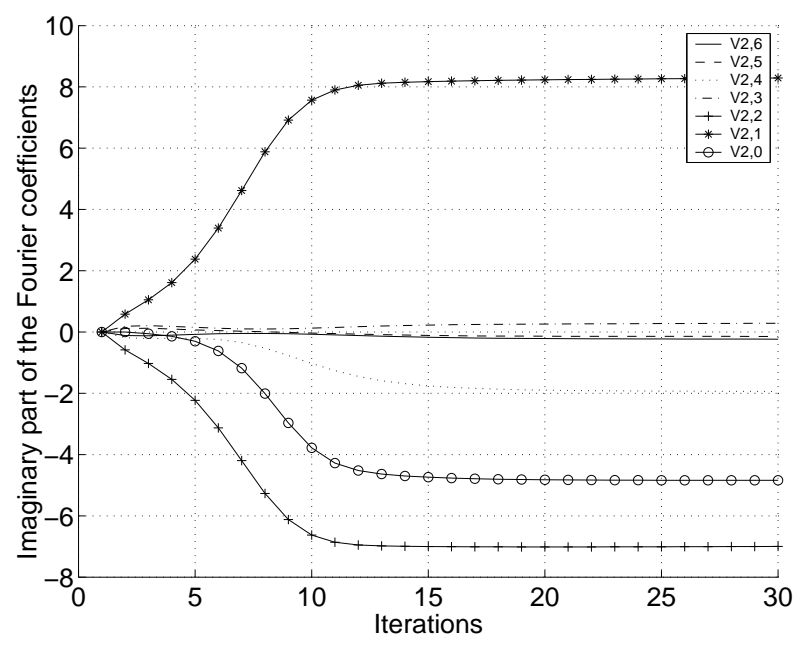

(b) Evolution of the imaginary part

Figure 41: Evolution of the Fourier coefficients for $v_{c 2}$ 


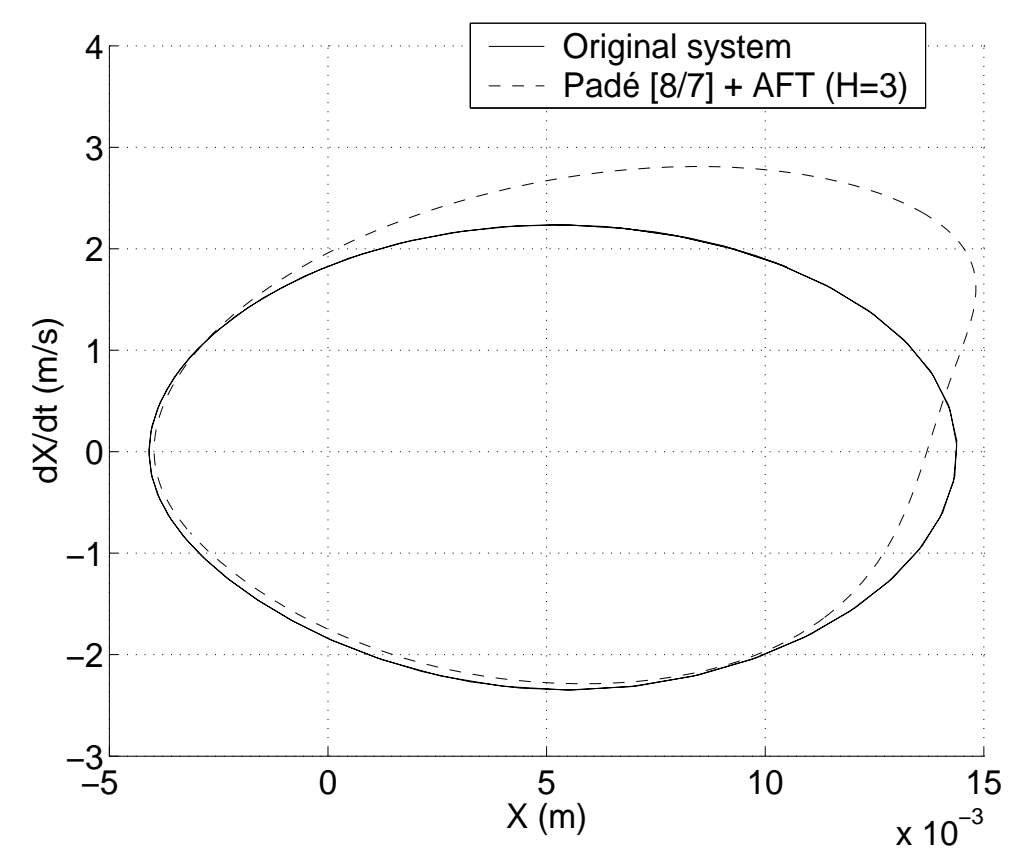

Figure 42: Limit cycle $(X, \dot{X})$ bu using an extension of the center manifold approach via the fractional approximants (for $\mu=1.01 \mu_{0}$ )

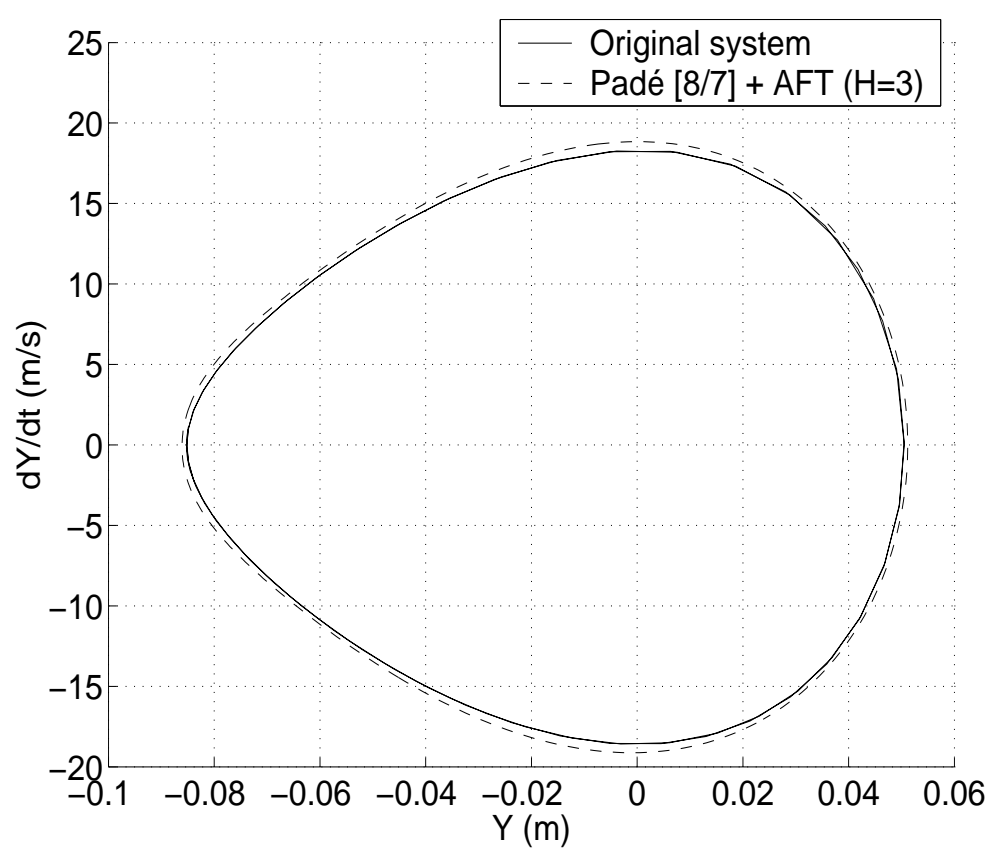

Figure 43: Limit cycle $(Y, \dot{Y})$ bu using an extension of the center manifold approach via the fractional approximants (for $\mu=1.01 \mu_{0}$ )

\subsection{Conclusion}

In this section, stability analysis, three non-linear methods and the associated computational techniques have been developed. First, the centre manifold approach was developed to reduce the number of degree-of-freedom of the original non-linear problem; a lower-dimensional system containing only the centre variables near the Hopf bifurcation point was defined. Second, the rational fractional approximants were used to simplify the non-linear terms. This new procedure consisting of applying the fractional approximants after the centre manifold was successfully applied: this new methodology extended the domain of validity of non-linear systems reduced by using the centre 
manifold approach. Moreover, it was demonstrated that one of the interesting aspects of these rational approximants is that they require fewer terms than the associated Taylor series to obtain an accurate approximation of the non-linear behaviour of the complete non-linear system. Even if the centre manifold approximation diverges or is insufficient to ap-proximate the non-linear solution near an equilibrium point, the associated rational approximants may converge and make it possible to obtain an approximation of the solution. This last advantage of the rational approximants is an extremely powerful property.

\section{Second example: a complex non-linear mechanical system}

In the previous section, the centre manifold, the rational fractional approximants and the Alternate Frequency/Time Domain method were used to reduce, to simplify and to obtain the final solution of a non-linear system with two degree-of-freedom possessing quadratic and cubic non-linearities as a truncated Fourier series approximation. The computational scheme of these methods were developed and the advantages of fractional approximants after the centre manifold approach were discussed; more particularly, the extension of the domain of validity by employing the fractional approximants and the possibility of obtaining an accurate solution even if the associated series expansion diverge was demonstrated.

A natural extension of this problem is to show the capability of this centre manifold extension via the fractional approximant for a more complex non-linear mechanical system such as an aircraft brake system where the problem of unstable vibrations in disk brakes has been studied by a number of researchers ([114], [9], [195], [68], [10], [63], [58], [32], [60], [127] and [68]).

As illustrated in Figure 44, an aircraft brake system is composed of a stack of rotating brake discs (rotors) which engage the wheel, and stationary brake discs (stators), which engage the torque tube. The torque tube attaches to the piston housing that links to the landing gear through a torque take-out rod. During operation, the brake is activated by hydraulic system pressure, which compresses the heat stack: the rotors and the stators are squeezed together by hydraulic pistons and the brake produces torque by virtue of friction forces generated at the rubbing interface between the rotors and the stators. Vibration can then be further induced by the friction characteristics of the heat sink material. Two important specific complex non-linear phenomena have been identified: squeal and whirl. The other major vibration modes are gear walk and chatter. Gear walk is defined as cyclic fore and aft motion of the landing gear assembly. The frequency spectrum of gear walk is in the $5-20 \mathrm{~Hz}$ range. Chatter is defined as a torsional motion of the rotating parts of the brake-wheel-tire assembly about the axle and against the elastic restraint of the tire. The frequency spectrum of chatter is in $50-100 \mathrm{~Hz}$ range. Squeal is defined as torsional vibrations of non-rotating brake parts around the axle. The frequency spectrum of squeal is in the $100-1000 \mathrm{~Hz}$ range. Whirl is defined as one vibration wherein the cantilevered end of the torque plaque orbits around the axle accompanied by un-phased pumping of the brake pistons. Brake whirl mode is within frequency range as brake squeal (200-300 Hz range) and can couple parametrically. Hydraulic damping provided by the piston housing fluid circuit provides a major source of whirl damping. If the hydraulic damping provided by the piston housing fluid is insufficient, orifices may be used to increase damping to required levels.

The goal of this section is to show the efficiency of the previous non-linear computational methods (the centre manifold approach, the fractional approximants and the Alternate Frequency/Time Domain method) for the stability analysis and complex non-linear behaviour of the whirl vibration in an aircraft brake system. First, a brief overview of friction induced vibration and some basic concepts of aircraft brake systems is presented in order to described the non-linear whirl model. Then, results from stability analyses and the extension of the centre manifold via rational approximants is investigated. 


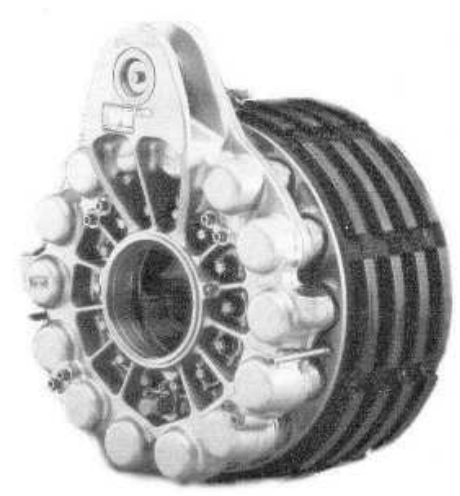

Figure 44: Aircraft brake system

\subsection{General presentation of the non-linear model}

\subsubsection{Overview of friction induced vibration mechanisms}

In this section, the general mechanisms of friction-induced vibration are briefly developed and more particularly the sprag-slip phenomenon and the associated geometric coupling is evaluated. Friction-induced vibration occurs in many industrial applications with rotating and sliding parts and is undesirable due to its detrimental effects on the performance of mechanical systems and its role in the accelerated wear of components, damage and noise. Different types of vibrations induced by friction have been studied in the past by several researchers ([124], [31], [79], [80], [38], [137], [49], [90], [51], [50], [150], [128], [199], [100], [76], [102], [105], [122], [123], [52] and [53]).

One of the important phases in studying vibration problems in systems is the determination of the mechanism of the unstable friction-induced vibration. There is no unique mathematical model and theory to explain the mechanisms and dynamic phenomena associated with friction: Ibrahim [79]-[80], Crolla and Lang [38] provide an extensive summary of many aspects of friction-induced vibration. Moreover, the contact forces between two surfaces play an important role in self-excited vibrations: Oden and Martins [137] proposed a review of frictional contact of metallic surfaces. The different mechanisms of friction-induced vibration fall into four classes: stick-slip, variable dynamic friction coefficient, sprag-slip and geometric coupling of degrees of freedom. In this study, we will consider the latter two approaches which use modal coupling to develop instability when the friction coefficient is constant. The first two approaches make use of the changes in the friction coefficient: the stick-slip is a low sliding speed phenomenon caused by the static friction coefficient being higher than the dynamic friction coefficient. The simple system which has been used to examine the stick-slip phenomenon, is that of a mass sliding on a moving belt. During the sliding phase, there is no change in the friction force that tends to make the mass stick on the moving belt. The sliding force increases until it exceeds the static friction force maximum. Consequently, the mass starts to slide. Next, the mass continues to slide until the force causing the sliding drops to the sliding friction value. Then, sliding and sticking occur in succession. Moreover, the speed dependence of kinematic friction was accepted to define the stick-slip motion and produce self-excited vibration ([59], [190], [16], [17], [4], [148], [166], [106], [11] and [205]).

The 1960s saw new developments of mechanisms for friction-induced vibration and the introduction of the motions of sprag-slip and geometric coupling. In 1961, Spurr [179] proposed this mechanism for friction induced vibrations. This approach uses kinematic constraints and modal coupling to develop instability. Following this work, several authors ([48], [136] and [121]) made contributions in support of this theory of geometric coupling: these studies have illustrated that frictional instability can be caused by geometrically induced instabilities that do not require variations in the coefficient of friction.

This notion of sprag-slip angle and geometric coupling will be considered to explain the whirl vibration in aircraft brake systems. Feld [58] explains the whirl vibration: the disks in the brake stack are compressed by the hydraulic pressure applied to the brake, as illustrated in Figure 45. Without vibration, the normal pressure is distributed uniformly over the rubbed surface between rotating and stationary disks. When vibration is present, disks in the brake stack are subjected to out-of-plane rotation called accordion motion. The uniform normal pressure over the disk interface is then altered by this accordion notion: the normal pressure increases over half of the interface and relaxes over the other half. Moreover, the friction force varies proportionally to this normal pressure and produces the whirl 
motion. On some gear systems, the brake rod attaches to the brake housing in an offset, cantilevered fashion such that the rod operates out-of-plane with the hydraulic system. Due to this offset, an angle may appear between the brake rod and the housing. This offset angle can be compared with the sprag-slip mechanism [179]. In this case, instability can occur with a constant brake friction coefficient. This angle in fact couples the normal and tangential contact force. This coupling is one of the primary cause of instability in the whirl vibration.

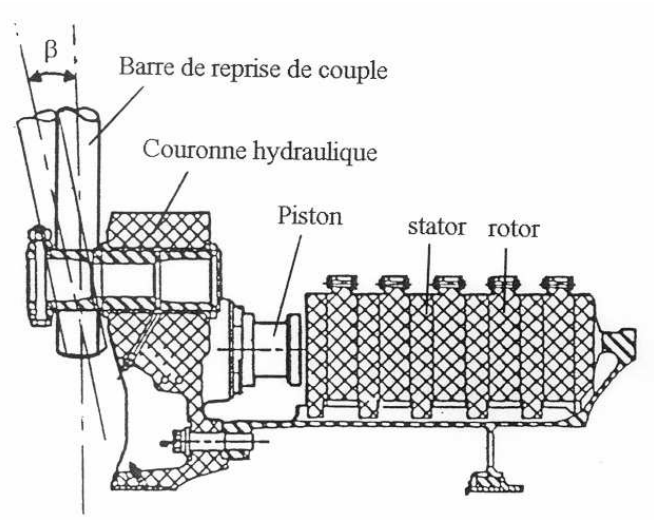

(a) Sprag-slip angle $\beta$

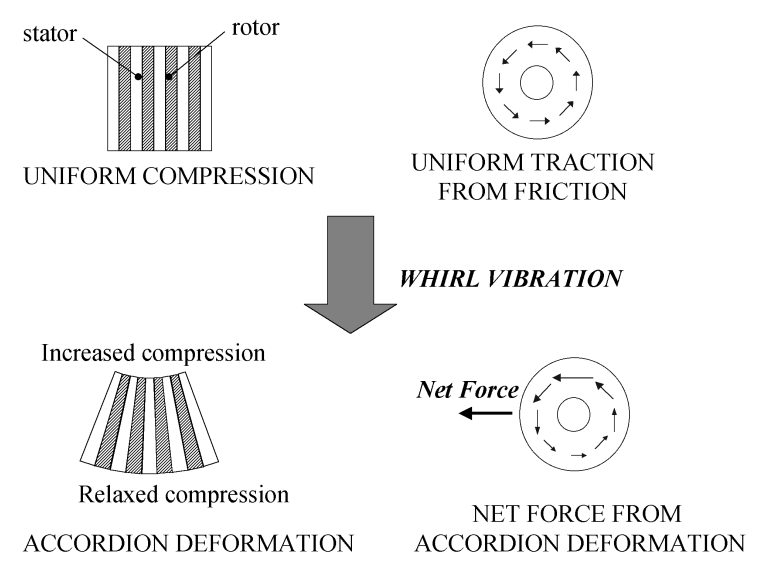

(b) Whirl vibration

Figure 45: Friction-induced whirl vibration

\subsubsection{Non-linear behaviour of the brake system}

In this system, the non-linear behaviour is due to the non-linear contact stress of the rotor-stator assembly. Experimental static tests show that the load-deflection relationship is highly non-linear, as illustrated in Figure 46 . We assume that the non-linear normal stress $N(r, \theta)$ acting at the interface surface between the stator and the rotor can be expressed as a cubic polynomial in the relative displacement between the rotor and stator in compression

$$
N(r, \theta)=\sum_{i=1}^{3} K_{i}(x(r, \theta))^{i}
$$

where $x(r, \theta)$ is the relative displacement between the rotor and the stator. $K_{1}, K_{2}$ and $K_{3}$ are the linear, quadratic and cubic coefficients of the non-linear contact between the rotor and the stator. This assumption is verified by static tests, as illustrated in Figure 46: the non-linear relationship between load and deflection is used to determine the coefficients $K_{1}, K_{2}$ and $K_{3}$. As shown in Figure 46, we have good agreement with the experimental non-linear contact stress and the cubic polynomial approximate solution. 


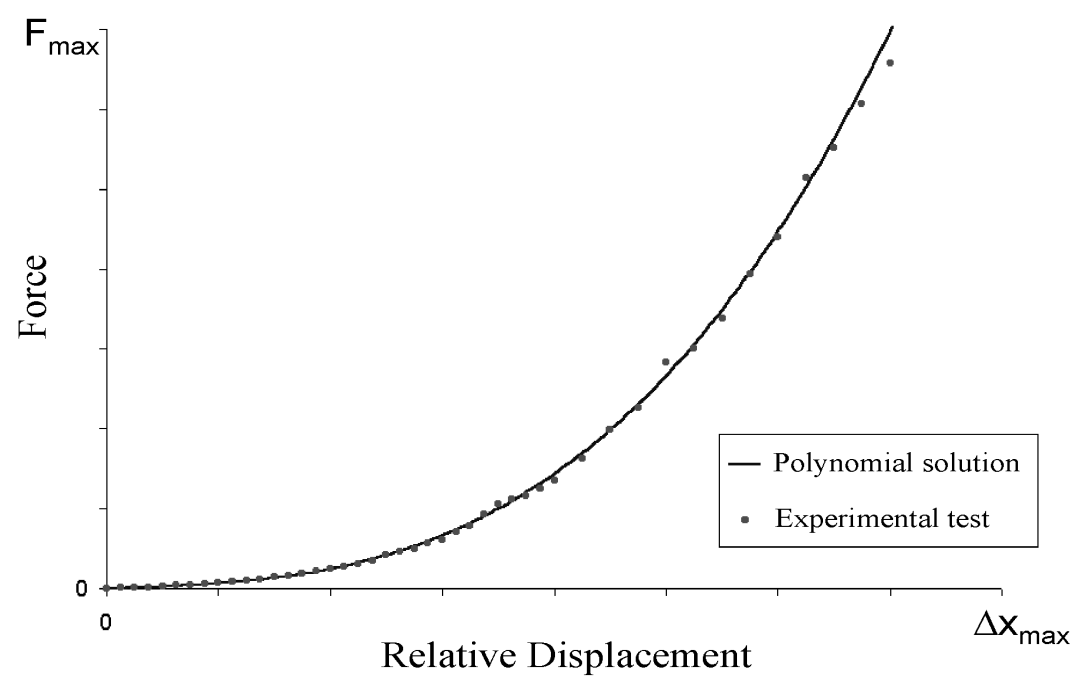

Figure 46: Non-linear contact stress

We then assume that the tangential stress $T$ is generated by the brake friction coefficient $\mu$, considering the Coulomb friction law $T(r, \theta)=\mu N(r, \theta)$. The multi-stage brake is represented by a single rotor, as illustrated in Figure 47, and stator with the effective brake friction coefficient $\mu_{b r k}=2 N \mu$ where $N$ defines the number of interfaces between stators and rotors. It is assumed that the rotor and stator friction surfaces are always in contact. In this whirl system, we consider the rigid body lateral displacement and the two yaws of the stator and rotor. For any point $M(r, \theta)$ on the rotor and stator, and by considering small displacements, the normal displacement of the rotor and the stator are

$$
\left\{\begin{array}{l}
x_{\text {rotor }}=x_{r}-r \sin \theta \sin \theta_{r}-r \cos \theta \sin \psi_{r} \\
x_{\text {stator }}=x_{s}-r \sin \theta \sin \theta_{s}-r \cos \theta \sin \psi_{s}
\end{array}\right.
$$

where $x_{r}, x_{s}, \theta_{r}, \theta_{s}, \psi_{s}$, and $\psi_{r}$ are the stator and the rotor lateral displacement, and the stator and rotor rotations, as illustrated in Figure 47. Then, for any point $M(r, \theta)$ on the disc surface, the normal displacement is

$$
x(r, \theta)=x_{\text {stator }}-x_{\text {rotor }}=\left(x_{s}-x_{r}\right)-r \sin \theta\left(\theta_{s}-\theta_{r}\right)-r \cos \theta\left(\psi_{s}-\psi_{r}\right)
$$

Next, by considering the non-linear normal stress expression of (185), the non-linear expressions of the normal force $F_{X}$ due to the normal contact between the rotor and the stator friction surface, and the non-linear expressions of the moments $M_{X}, M_{Y}$ and $M_{Z}$ are given by

$$
\begin{aligned}
F_{X}= & \int_{0}^{2 \pi} \int_{R_{i}}^{R_{e}} P(M) r d r d \theta \\
= & K_{1} A_{2}\left(x_{s}-x_{r}\right)+K_{2}\left(A_{2}\left(x_{s}-x_{r}\right)^{2}+\frac{A_{4}}{4}\left(\theta_{s}-\theta_{r}\right)^{2}+\frac{A_{4}}{4}\left(\psi_{s}-\psi_{r}\right)^{2}\right) \\
& \quad+K_{3}\left(A_{2}\left(x_{s}-x_{r}\right)^{3}+\frac{3 A_{4}}{4}\left(\theta_{s}-\theta_{r}\right)^{2}\left(x_{s}-x_{r}\right)+\frac{3 A_{4}}{4}\left(\psi_{s}-\psi_{r}\right)^{2}\left(x_{s}-x_{r}\right)\right) \\
M_{X}=\quad & \int_{0}^{2 \pi} \int_{R_{i}}^{R_{e}} 2 N \mu_{b r k} P(M) r^{2} d r d \theta \\
= & 2 N \mu_{b r k}\left(K_{1} \frac{2 A_{3}}{3}\left(x_{s}-x_{r}\right)+K_{2}\left(\frac{2 A_{3}}{3}\left(x_{s}-x_{r}\right)^{2}+\frac{A_{5}}{5}\left(\theta_{s}-\theta_{r}\right)^{2}+\frac{A_{5}}{5}\left(\psi_{s}-\psi_{r}\right)^{2}\right)\right. \\
+ & \left.K_{3}\left(\frac{2 A_{3}}{3}\left(x_{s}-x_{r}\right)^{3}+\frac{3 A_{5}}{5}\left(x_{s}-x_{r}\right)\left(\theta_{s}-\theta_{r}\right)^{2}+\frac{3 A_{5}}{5}\left(x_{s}-x_{r}\right)\left(\psi_{s}-\psi_{r}\right)^{2}\right)\right) \\
M_{Y}= & -\int_{0}^{2 \pi} \int_{R_{i}}^{R_{e}} P(r, \theta) r^{2} \sin \theta d r d \theta \\
= & -K_{1} \frac{A_{4}}{4}\left(\theta_{s}-\theta_{r}\right)-K_{2} \frac{A_{4}}{2}\left(\theta_{s}-\theta_{r}\right)\left(x_{s}-x_{r}\right) \\
& -K_{3}\left(\frac{3 A_{4}}{4}\left(\theta_{s}-\theta_{r}\right)\left(x_{s}-x_{r}\right)^{2}+\frac{A_{6}}{8}\left(\theta_{s}-\theta_{r}\right)^{3}+\frac{A_{6}}{8}\left(\theta_{s}-\theta_{r}\right)\left(\psi_{s}-\psi_{r}\right)^{2}\right)
\end{aligned}
$$




$$
\begin{aligned}
M_{Z}= & -\int_{0}^{2 \pi} \int_{R_{i}}^{R_{e}} P(r, \theta) r^{2} \cos \theta d r d \theta \\
= & -K_{1} \frac{A_{4}}{4}\left(\psi_{s}-\psi_{r}\right)-K_{2} \frac{A_{4}}{2}\left(\psi_{s}-\psi_{r}\right)\left(x_{s}-x_{r}\right) \\
& -K_{3}\left(\frac{3 A_{4}}{4}\left(\psi_{s}-\psi_{r}\right)\left(x_{s}-x_{r}\right)^{2}+\frac{A_{6}}{8}\left(\psi_{s}-\psi_{r}\right)^{3}+\frac{A_{6}}{8}\left(\psi_{s}-\psi_{r}\right)\left(\theta_{s}-\theta_{r}\right)^{2}\right)
\end{aligned}
$$

with $A_{k}=\pi\left(R_{e}^{k}-R_{i}^{k}\right)$ for $k=1,2,3,4, \ldots, 6$.
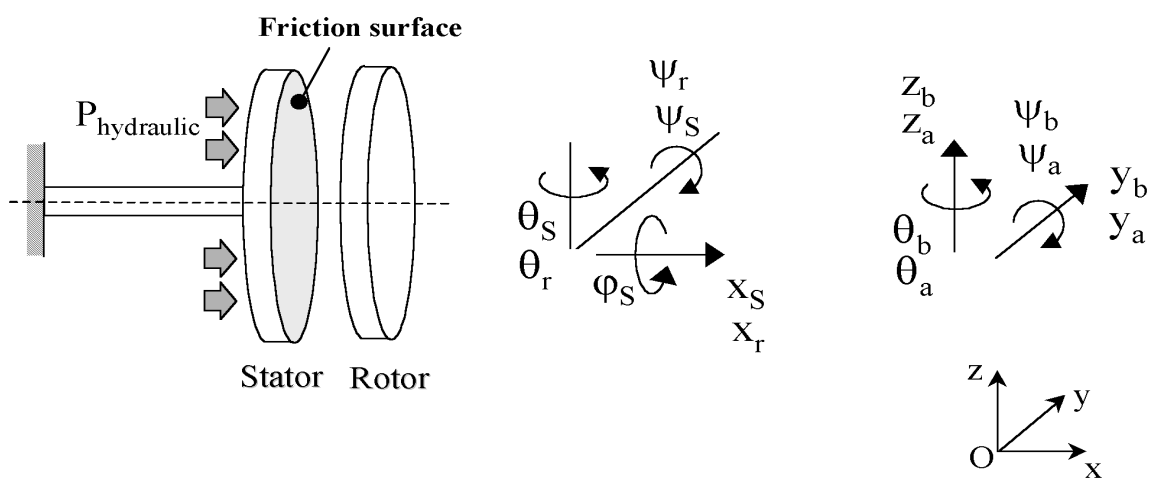

Figure 47: Model of the whirl vibration

\subsubsection{Equations of the non-linear dynamical system}

The equations of motion for the non-linear aircraft brake system are [167]

$$
\begin{aligned}
& m_{s} \ddot{x}_{s}+C_{x s} \dot{x_{s}}=F_{b a r r e / X}+F_{h y d / X}-F_{X} \\
& I_{\theta s} \ddot{\theta}_{s}+C_{\theta s} \dot{\theta}_{s}+C_{t w k}\left(\dot{\theta_{s}}-\dot{\theta}_{t}\right)+K_{\theta s} \theta_{s}+K_{t w k}\left(\theta_{s}-\theta_{t}\right)=F_{b a r r e} / X R_{e}+F_{b a r r e} / Z d_{e}+M_{Y} \\
& I_{\psi s} \ddot{\psi}_{s}+C_{\psi s} \dot{\psi}_{s}+C_{t w k}\left(\dot{\psi}_{s}-\dot{\psi}_{t}\right)+K_{\psi s} \psi_{s}+K_{t w k}\left(\psi_{s}-\psi_{t}\right)=F_{b a r r e} / Y d_{e}+M_{Z} \\
& I_{\phi s} \ddot{\phi}_{s}+C_{\phi s} \dot{\phi}_{s}=-F_{\text {barre } / Z} \sin \alpha R_{e}-F_{\text {barre } / Y} R_{e} \cos \alpha+M_{X} \\
& m_{r}{\ddot{x_{r}}}+C_{r r} \dot{x_{r}}+K_{r r} x_{r}=F_{X} \\
& I_{\theta r} \ddot{\theta_{r}}+C_{\theta r} \dot{\theta_{r}}+C_{f w k}\left(\dot{\theta_{r}}-\dot{\theta_{f}}\right)+K_{f w k}\left(\theta_{r}-\theta_{f}\right)=-M_{Y} \\
& I_{\psi r} \ddot{\psi}_{r}+C_{\psi r} \dot{\psi}_{r}+C_{f w k}\left(\dot{\psi}_{r}-\dot{\psi}_{f}\right)+K_{f w k}\left(\psi_{r}-\psi_{f}\right)=-M_{Z} \\
& m_{f} \ddot{y}_{f}+C_{f 11} \dot{y}_{f}+C_{y t f}\left(\dot{y}_{f}-\dot{y}_{t}\right)+K_{f 11} y_{f}+K_{f 12} \theta_{f}+K_{y t f}\left(y_{f}-y_{t}\right)=0 \\
& I_{f} \ddot{\theta_{f}}+C_{f 22} \dot{\theta_{f}}+C_{f w k}\left(\dot{\theta_{f}}-\dot{\theta_{r}}\right)+C_{\theta t f}\left(\dot{\theta_{f}}-\dot{\theta}_{t}\right)+K_{f 21} y_{f}+K_{f 22} \theta_{f}+K_{f w k}\left(\theta_{f}-\theta_{r}\right)+K_{\theta t f}\left(\theta_{f}-\theta_{t}\right)=0 \\
& m_{f} \ddot{z}_{f}+C_{f 11} \dot{z}_{f}+C_{z t f}\left(\dot{z}_{f}-\dot{z}_{t}\right)+K_{f 11} z_{f}+K_{f 12} \psi_{f}+K_{z t f}\left(z_{f}-z_{t}\right)=0 \\
& I_{f} \ddot{\psi}_{f}+C_{f 22} \dot{\psi_{f}}+C_{f w k}\left(\dot{\psi}_{f}-\dot{\psi}_{r}\right)+C_{\psi t f}\left(\dot{\psi}_{f}-\dot{\psi}_{t}\right)+K_{f 21} z_{f}+K_{f 22} \psi_{f}+K_{f w k}\left(\psi_{f}-\psi_{r}\right)+K_{\psi t f}\left(\psi_{f}-\psi_{t}\right)=0 \\
& m_{t} \ddot{y}_{t}+C_{t 11} \dot{y}_{t}+C_{y t f}\left(\dot{y}_{t}-\dot{y}_{f}\right)+K_{t 11} y_{t}+K_{t 12} \theta_{t}+K_{y t f}\left(y_{t}-y_{f}\right)=0 \\
& I_{t} \ddot{\theta}_{t}+C_{f 22} \dot{\theta}_{t}+C_{f w k}\left(\dot{\theta}_{t}-\dot{\theta_{s}}\right)+C_{\theta t f}\left(\dot{\theta}_{t}-\dot{\theta_{f}}\right)+K_{t 21} y_{t}+K_{t 22} \theta_{t}+K_{t w k}\left(\theta_{t}-\theta_{s}\right)+K_{\theta t f}\left(\theta_{t}-\theta_{f}\right)=0 \\
& m_{t} \ddot{z}_{t}+C_{t 11} \dot{z}_{t}+C_{z t f}\left(\dot{z}_{t}-\dot{z}_{f}\right)+K_{t 11} z_{t}+K_{t 12} \psi_{t}+K_{z t f}\left(z_{t}-z_{f}\right)=0
\end{aligned}
$$

where $x_{s}, x_{r}, \theta_{s}, \theta_{r}, \psi_{s}, \psi_{s}, \phi_{s}, y_{f}, y_{t}, z_{f}, z_{t}, \psi_{f}, \psi_{t}, \theta_{f}$ and $\theta_{t}$ are the stator and the rotor lateral displacement, the stator and rotor rotations, the piston torsional rotation and the axle deflections and rotations of the rotor and stator shaft, respectively. The stator and the shaft of the stator interact via notches on the inner perimeter of the disk, and the 
rotor and the shaft of the rotor interact via drive keys on the outside of the disk. $F_{h y d / X}$ defines the brake force from hydraulic pressure that is given by

$$
F_{\text {hyd/X }}=\frac{6\left(D_{\text {piston/outer }}^{2}-D_{\text {piston/inner }}^{2}\right)}{\left(D_{\text {disque/outer }}^{2}-D_{\text {disque/inner }}^{2}\right)} P_{\text {hydraulic }} n_{\text {piston }}
$$

where $n_{\text {piston }}, D_{\text {piston/outer }}, D_{\text {piston/inner }}$ are the number of pistons, the outer and inner diameter of the piston surface in contact with the stator, respectively. $D_{\text {disque/outer }}$ and $D_{\text {disque/inner }}$ define the outer and inner diameter of the rotor-stator interface, respectively.

$K_{t w k}$ and $C_{t w k}$ define the stiffness and the damping between the stator and the shaft of the stator, called torque tube, via notches on the inner perimeter of the disk. $K_{f w k}$ and $C_{f w k}$ define the stiffness and the damping between the rotor and the shaft of the rotor, via drive keys on the outside of the disk. $K_{\psi t f}, K_{\theta t f}, K_{y t f}, K_{z t f}$ and $C_{\psi t f}, C_{\theta t f}, C_{y t f}$, $C_{z t f}$ are the contact stiffness and the contact damping between the rotor's and stator's shaft, respectively. $K_{r r}$ defines the stiffness of the back-plate of the brake. $K_{t i j}$ and $C_{t i j}$ (for $1 \leq i \leq 2$ and $1 \leq j \leq 2$ ) are the axle bend stiffness and axle bend damping for the stator's shaft, respectively. $K_{f i j}$ and $C_{f i j}$ (for $1 \leq i \leq 2$ and $1 \leq j \leq 2$ ) are the axle bend stiffness and axle bend damping for the rotor's shaft, respectively. $d_{e}$ and $R_{e}$ represent the brake rod lateral offset and the distance axle to brake rod axis. $F_{X}, M_{X}, M_{Y}$ and $M_{Z}$ are the normal contact between the rotor and the stator friction surfaces and the associated moments, respectively. $F_{\text {couple } / X}, F_{\text {couple } / Y}$ and $F_{\text {couple } / Z}$ define the load due to the brake rod. We have

$$
\left\{\begin{array}{l}
F_{\text {couple } / X}=K_{\text {couple }} R_{e} \phi_{s} \cos \alpha \sin \beta+K_{\text {couple }} x_{s} \sin \beta+K_{\text {couple }} R_{e} \sin \beta\left(\theta_{s} \cos \alpha+\psi_{s} \sin \alpha\right) \\
F_{\text {couple } / Y}=K_{\text {couple }} R_{e} \phi_{s} \cos \alpha \cos \beta-K_{\text {couple }} d_{e} \theta_{s} \cos \beta \\
F_{\text {couple } / Z}=K_{\text {couple }} R_{e} \phi_{s} \sin \alpha \cos \beta-K_{\text {couple }} d_{e} \psi_{s} \cos \beta
\end{array}\right.
$$

where $K_{\text {couple }}$ defines the axial stiffness of the brake rod and $\alpha$ the sprag-slip angle due to the brake rod angle offset with the rotor-stator interface. It may be observed that the friction-induced vibration is only due to the fact that this angle $\alpha$ is not equal zero.

Finally, the complex non-lniear equations may be rewritten in the following form

$$
\mathbf{M} \ddot{\mathbf{x}}+\mathbf{C} \dot{\mathbf{x}}+\mathbf{K x}=\mathbf{F}_{\text {hydraulic }}(\mathbf{x})+\mathbf{F}_{\text {couple }}(\mathbf{x})+\mathbf{F}_{\text {friction }}(\mathbf{x})
$$

with

$$
\mathbf{x}=\left\{\begin{array}{lllllllllllllll}
x_{s} & \theta_{s} & \psi_{s} & \phi_{s} & x_{r} & \theta_{r} & \psi_{r} & y_{f} & \theta_{f} & z_{f} & \psi_{f} & y_{t} & \theta_{t} & z_{t} & \psi_{t}
\end{array}\right\}^{T}
$$

where $\mathbf{M}, \mathbf{C}$ and $\mathbf{K}$ are the $15 \times 15$ mass, damping and stiffness matrices, respectively. $\mathbf{F}_{\text {hydraulic }}$ is the vector force due to net brake hydraulic pressure in the $\mathrm{x}$-basis; $\mathbf{F}_{\text {couple }}$ is the vector due to the brake rod load, and $\mathbf{F}_{\text {friction }}$ contains the linear and non-linear frictional contact force terms at the stator and rotor interface and in the $\mathrm{x}$-basis.

We easily observe that $\mathbf{F}_{\text {couple }}$ is described as a linear expression versus the vector $\mathbf{x}$ :

$$
\mathbf{F}_{\text {couple }}(\mathbf{x})=\mathbf{K}_{\text {couple }} \mathbf{x}
$$

The set of equations describing the dynamic of the non-linear system may then be written as

$$
\mathbf{M} \ddot{\mathbf{x}}+\mathbf{C} \dot{\mathbf{x}}+\mathbf{K}_{\mathbf{t}} \mathbf{x}=\mathbf{F}_{\text {hydraulic }}(\mathbf{x})+\mathbf{F}_{\text {friction }}(\mathbf{x})
$$

with

$$
\mathbf{K}_{\mathbf{t}}=\mathbf{K}-\mathbf{K}_{\text {couple }}
$$

As explained previously, the non-linear analysis can be divided into two parts. First, the stability analysis is investigated by estimating the equilibrium point and by considering the eigenvalues of the jacobian matrix of the linearized system at this equilibrium point. Second, the dynamic characteristics of friction induced vibration in the aircraft brake system near the Hopf bifurcation point is investigated. The main purpose of this last section is to consider non-linear methods to reduce the non-linear mechanical system for instability computation. The computational procedure of the centre manifold extension by using the fractional approximants and the Alternate Frequency/Time Domain method, is applied to obtain a reduced and simplified non-linear system retaining the essential features of the non-linear dynamic behaviour near the Hopf bifurcation point. 


\subsection{Stability analysis}

As explained in Section 3.2, to study the stability of the system, the equilibrium point of the non-linear system is investigated and then the non-linear equations of motion are linearized around each steady-state equilibrium position. The equilibrium point $\mathbf{x}_{\mathbf{0}}$ is obtained by solving the non-linear static equations for a given net brake hydraulic pressure

$$
\mathbf{K}_{\mathbf{t}} \mathbf{x}_{\mathbf{0}}=\mathbf{F}_{\text {hydraulic }}\left(\mathbf{x}_{\mathbf{0}}\right)+\mathbf{F}_{\text {friction }}\left(\mathbf{x}_{\mathbf{0}}\right)
$$

Next, the stability analysis is investigated around the steady-state operating point by assuming small perturbations $\overline{\mathbf{x}}$ around the equilibrium point $\mathbf{x}_{\mathbf{0}}$ (with $\mathbf{x}=\mathbf{x}_{\mathbf{0}}+\overline{\mathbf{x}}$ ). The frictional contact vector $\mathbf{F}_{\text {friction }}$ may be expressed in terms of both equilibrium position $\mathbf{x}_{\mathbf{0}}$ and perturbation variables $\overline{\mathbf{x}}$, where

$$
\mathbf{F}_{\text {friction }}(\mathbf{x})=\mathbf{F}_{\text {friction }}\left(\mathbf{x}_{\mathbf{0}}\right)+\mathbf{F}_{\text {friction }}(\overline{\mathbf{x}})
$$

By substituting (215) in (214) and by considering only the linear terms $\mathbf{F}_{\text {friction }}^{\mathbf{L}}(\overline{\mathbf{x}})$, on has

$$
\mathbf{M} \ddot{\overline{\mathbf{x}}}+\mathbf{C} \dot{\overline{\mathbf{x}}}+\mathbf{K}_{\mathbf{t}} \overline{\mathbf{x}}=\mathbf{F}_{\text {friction }}^{\mathrm{L}}(\overline{\mathbf{x}})
$$

with

$$
\mathbf{F}_{\text {friction }}^{\mathbf{L}}(\overline{\mathbf{x}})=\left.\sum_{i=1}^{15} \frac{\partial \mathbf{F}_{\text {friction }}(\overline{\mathbf{x}})}{\partial \overline{x_{i}}}\right|_{\mathbf{x}_{\mathbf{0}}} \overline{x_{i}}
$$

The final expression of the vector $\mathbf{F}_{\text {friction }}^{\mathbf{L}}$ is

$$
\mathbf{F}_{\text {friction }}^{\mathbf{L}}=\left\{\begin{array}{llllllllllllllll}
-F_{X}^{L} & M_{Y}^{L} & M_{Z}^{L} & M_{X}^{L} & F_{X}^{L} & -M_{Y}^{L} & -M_{Z}^{L} & 0 & 0 & 0 & 0 & 0 & 0 & 0 & 0
\end{array}\right\}^{T}
$$

The analytical linear expressions of the terms $F_{X}^{L}, M_{Y}^{L}, M_{Z}^{L}$ and $M_{X}^{L}$ in terms of both equilibrium position $\mathrm{x}_{0}$ and perturbation variables $\overline{\mathbf{x}}$ are given in Annexe $\mathrm{B}$.

Finally, the computational stability analysis can be performed on the eigenvalues of the matrix $\mathbf{A}$

$$
\mathbf{A}=\left[\begin{array}{cc}
0 & \mathbf{I} \\
-\mathbf{M}^{-\mathbf{1}}\left(\mathbf{K}_{\mathbf{t}}-\mathbf{K}_{\text {friction }}^{\mathbf{L}}\right) & -\mathbf{M}^{-\mathbf{1}} \mathbf{C}
\end{array}\right]
$$

Results from computational stability analyses are presented in Figures 48-49. A representation of the evolution of frequencies and the evolution of the associated real part against brake friction coefficient $\mu$ are given in Figures 48, and Figures 49, respectively. A representation of the evolution of the eigenvalues in the complex plane is presented in Figure 49. As long as the real part of all the eigenvalues remains negative, the system is stable. When at least one of the eigenvalues has a positive real part, the dynamical system is unstable. Generally the system is stable at low value of brake friction coefficient $\mu$ and unstable at high values. The frequency of instability is obtained near $250 \mathrm{~Hz}$ : a perfect correlation with experimental tests where the frequency of instability near $260 \mathrm{~Hz}$ is obtained. Moreover, the mode shape obtained in association with this instability defines a wobbling motion between the brake's rotating and stationary parts and we observe that in this mode the cantilevered end of the torque plaque orbits around the axle, as observed experimentally. To illustrate the high potential and efficiency of the computational stability analysis, parametric studies are presented: Figures 50-52 show the evolution of the real and imaginary parts of eigenvalues with various brake friction coefficients $\mu$, brake hydraulic pressures $P$ and sprag-slip angle $\alpha$. 


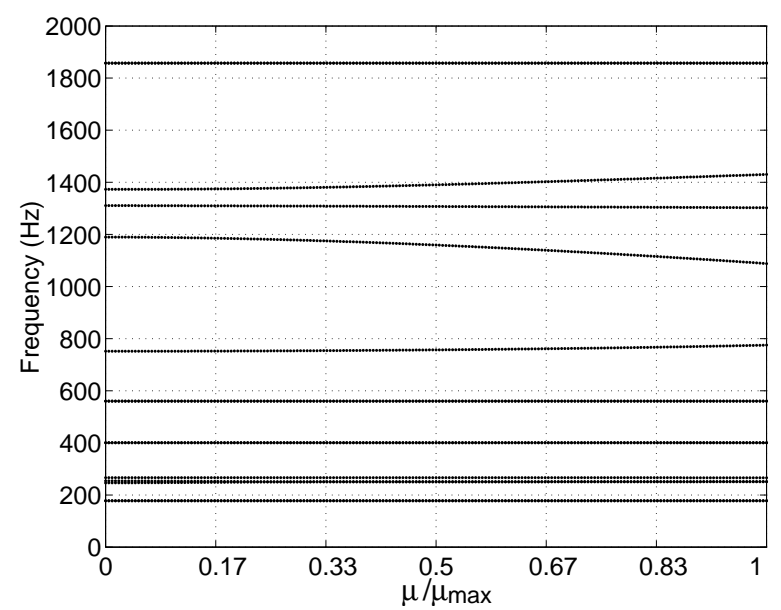

(a) Evolution of frequencies

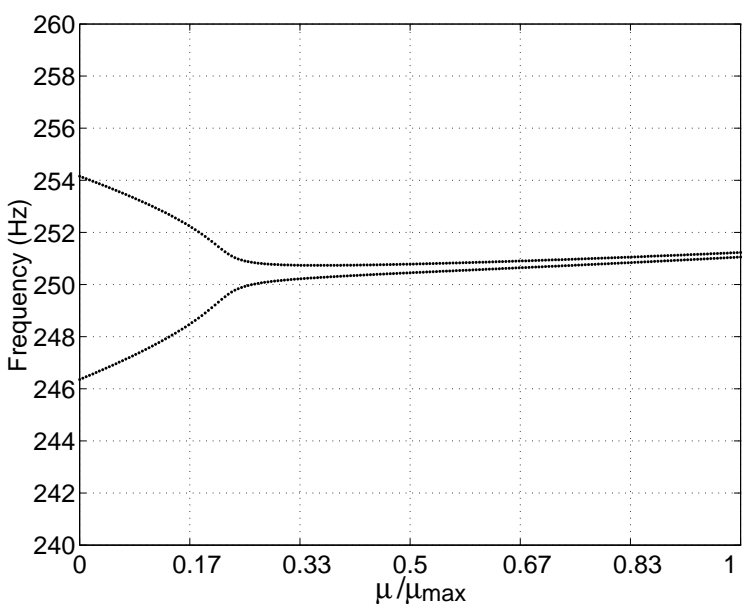

(b) Zoom

Figure 48: Evolution of all the frequencies and coupling modes

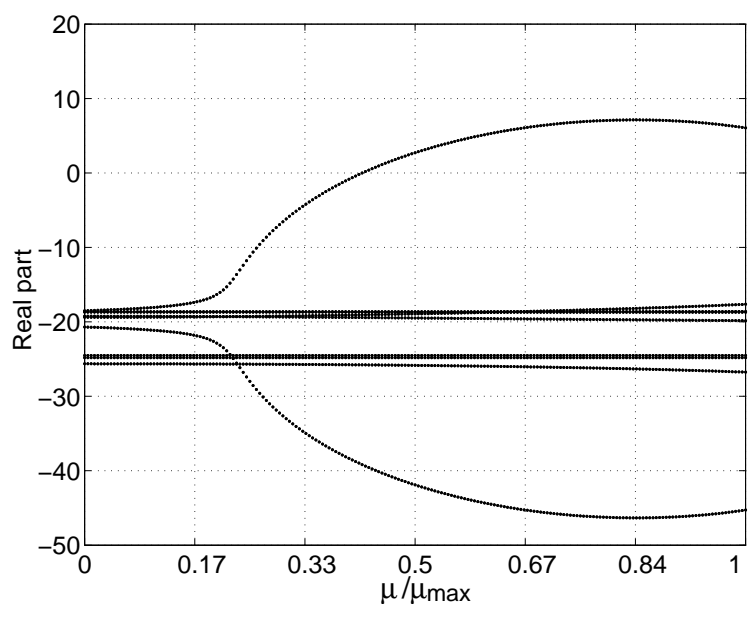

(a) Evolution of the real parts

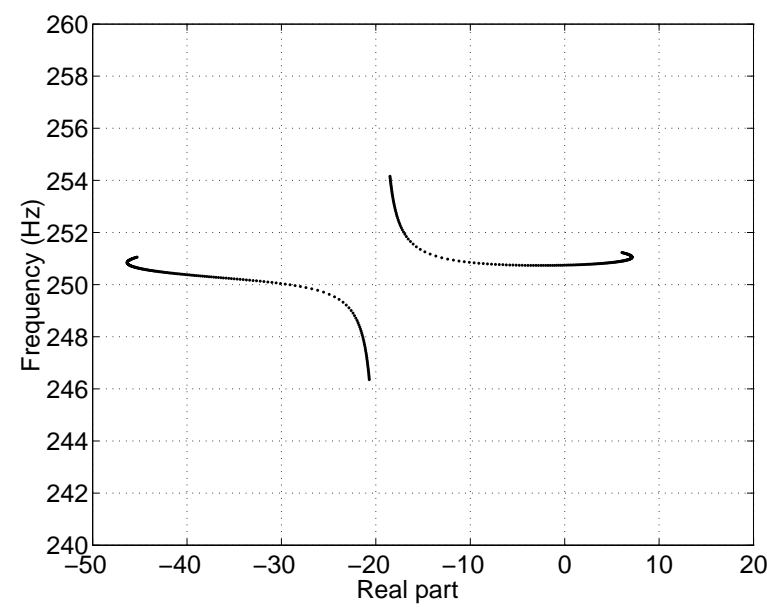

(b) Evolution of the eigenvalues in the complex plane

Figure 49: Evolution of the real parts and of the eigenvalues in the complex plane

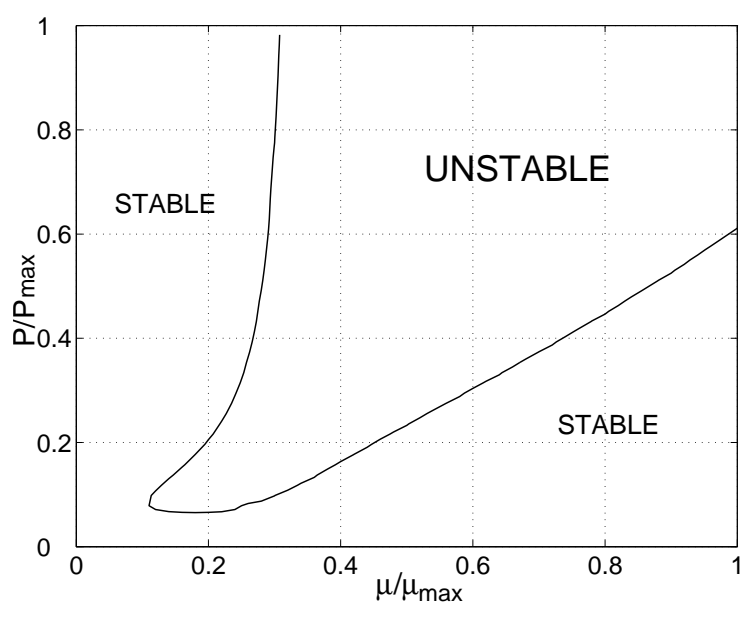

(a) Stable/Unstable areas

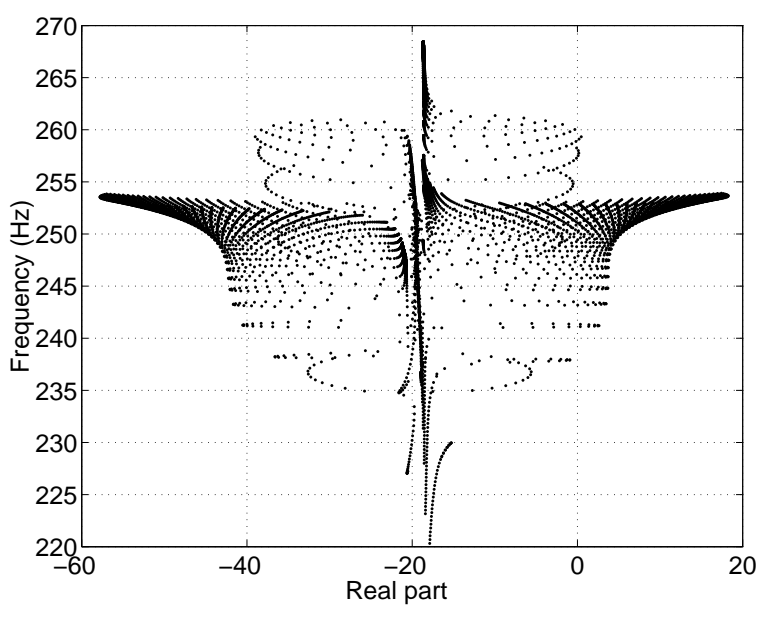

(b) Evolution of frequencies

Figure 50: Stability analysis versus the friction coefficient $\mu$ and the pressure $P$ 


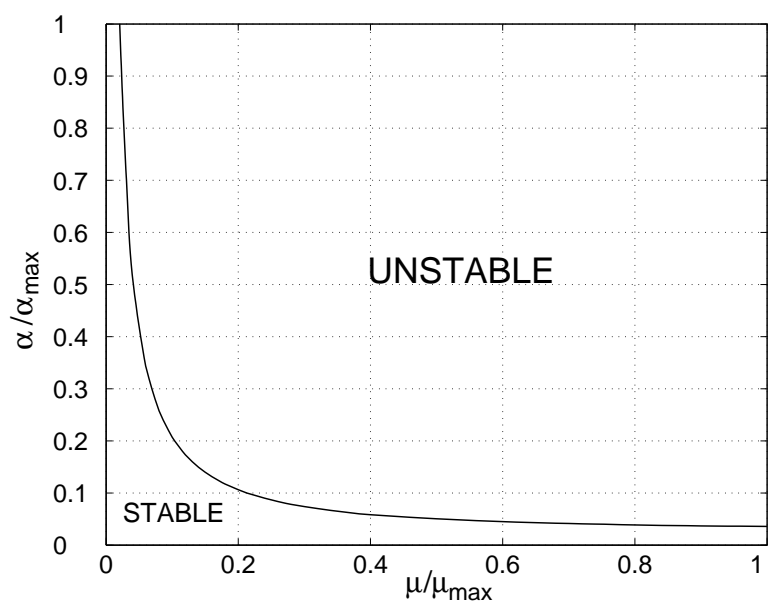

(a) Stable/Unstable areas

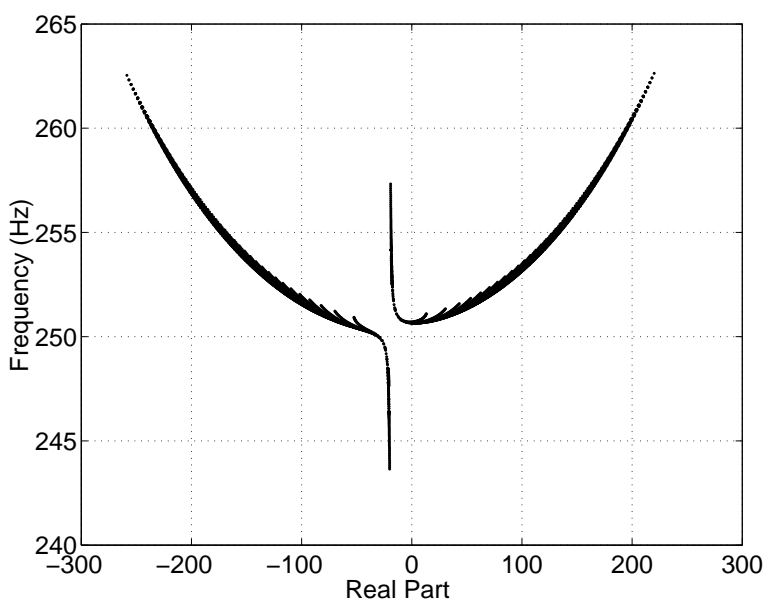

(b) Evolution of frequencies

Figure 51: Stability analysis versus the friction coefficient $\mu$ and the sprag-slip angle $\alpha$

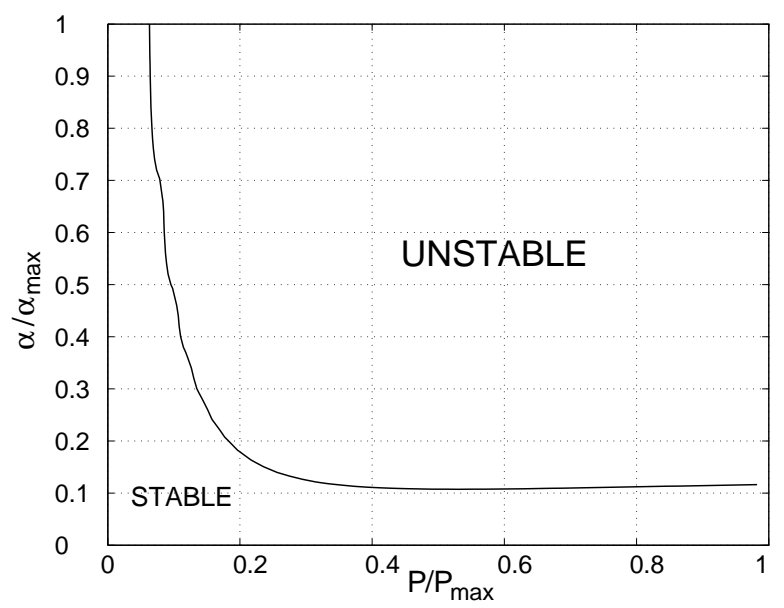

(a) Stable/Unstable areas

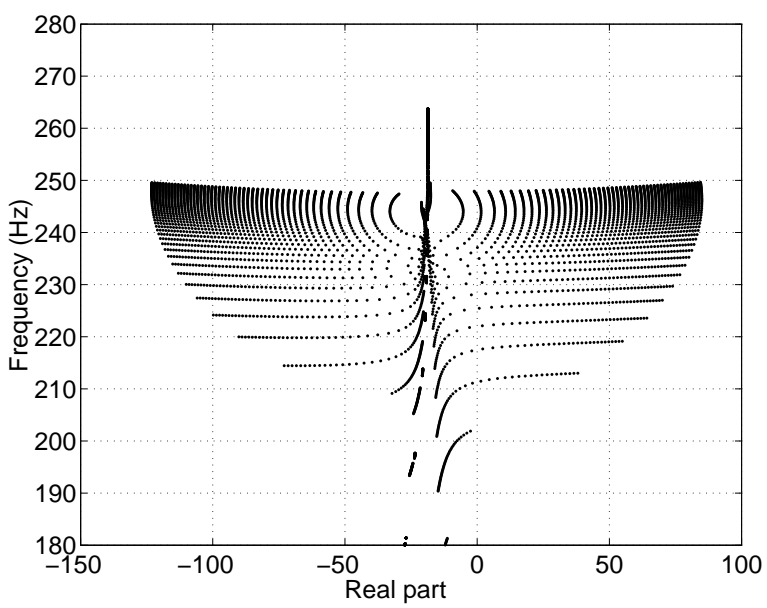

(b) Evolution of frequencies

Figure 52: Stability analysis versus the pressure $P$ and the sprag-slip angle $\alpha$

\subsection{Non-linear dynamics}

The time-history responses of the non-linear dynamical system (212) is first calculated by using classical $4^{\text {th }}$-order Runge-Kutta algorithm, as illustrated in Figures 53- 55. However, this procedure is rather expensive and consumes considerable resources both in terms of the computation time and in terms of the data storage. Understanding the behaviour of this non-linear system thus requires simplification and reduction of the equations. In order to obtain the non-linear simplified system, the computational extension of the centre manifold approach using the rational fractional approximants and the Alternate/Frequency Time domain method will be applied. 


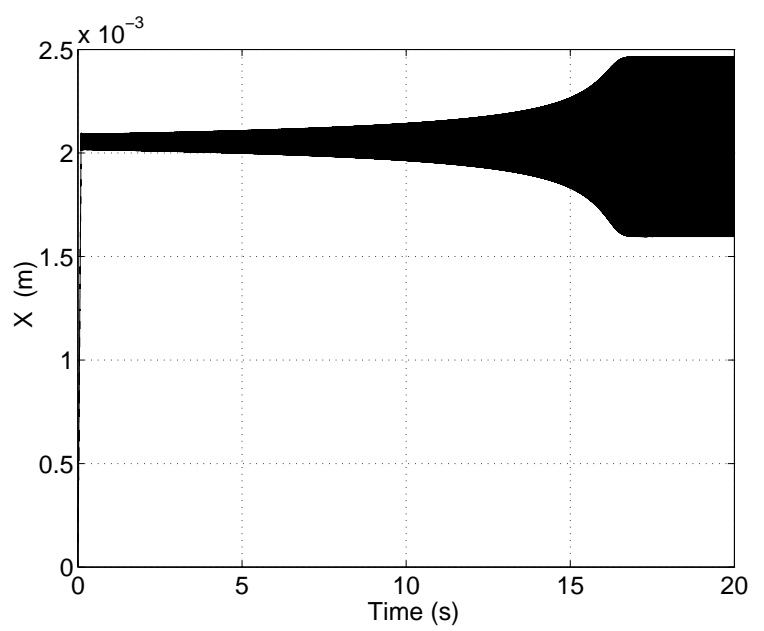

(a) Displacement $x_{s}(t)$

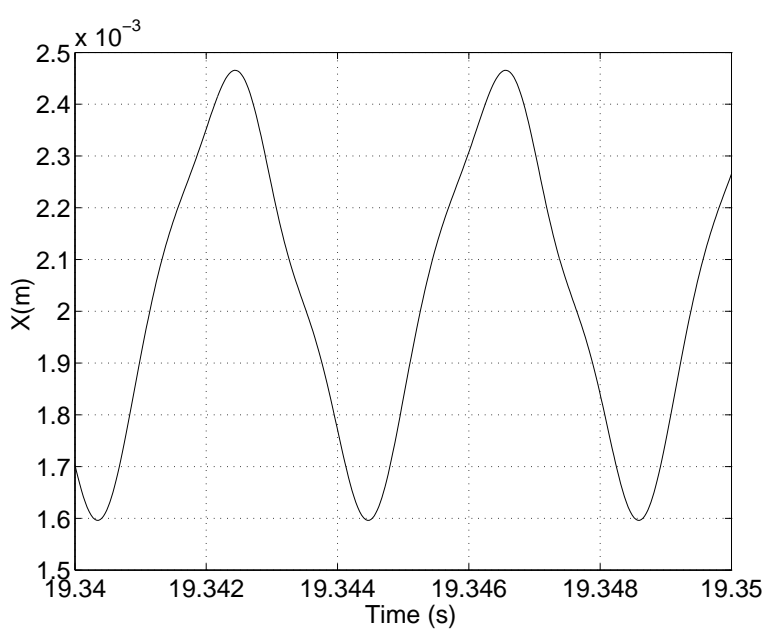

(b) Zoom

Figure 53: Non-linear dynamic of the displacement $x_{s}(t)$ for $\mu=1.01 \mu_{0}$

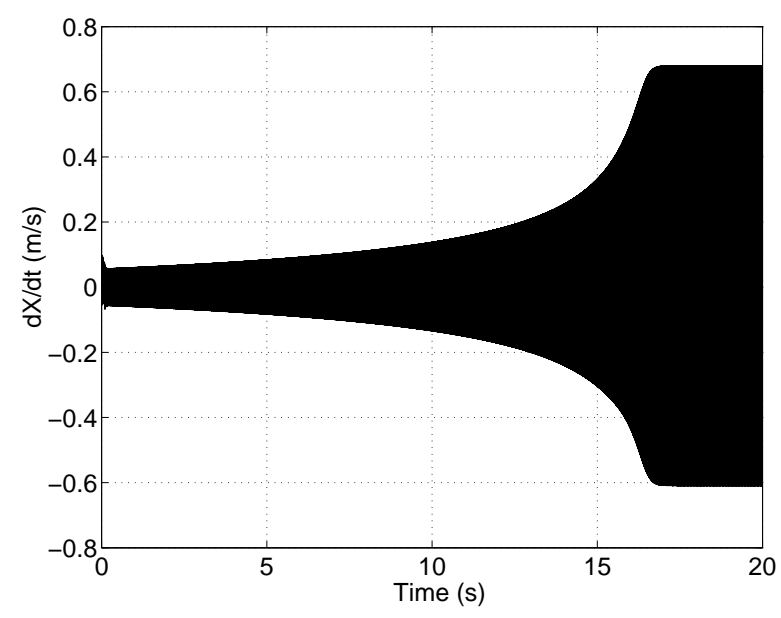

(a) Velocity $\dot{x_{s}}(t)$

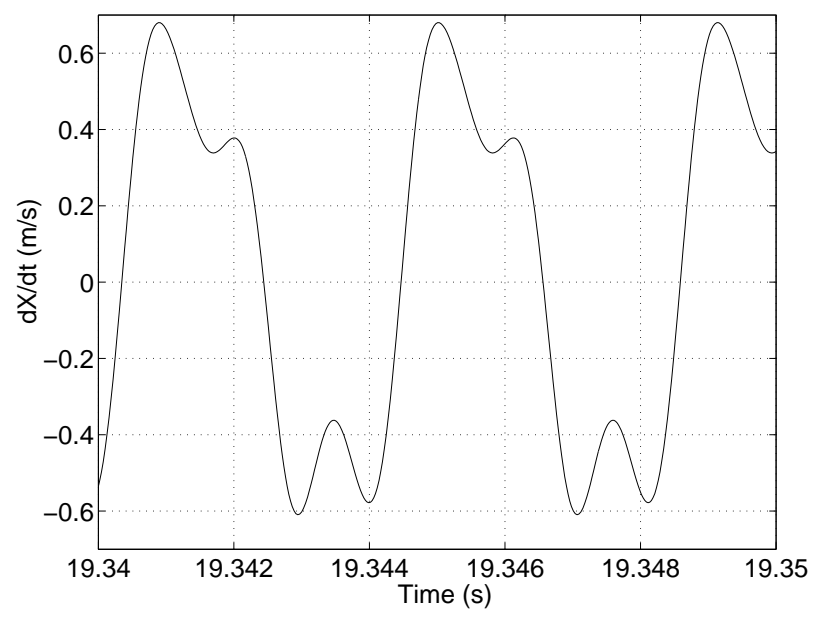

(b) Zoom

Figure 54: Non-linear dynamic of the velocity $\dot{x_{s}}(t)$ for $\mu=1.01 \mu_{0}$ 


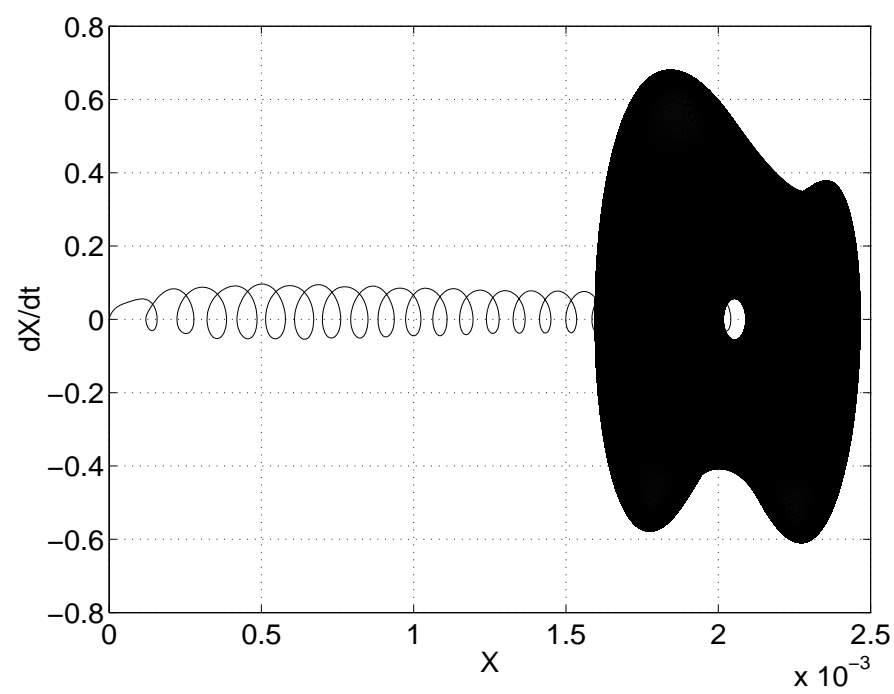

Figure 55: Limit cycle $\left(x_{s}, \dot{x_{s}}\right)$ for $\mu=1.01 \mu_{0}$

\subsubsection{Results via the computational centre manifold extension via the fractional approximants}

By considering (212), the complete non-linear expressions of the non-linear forces are expressed in order to conduct this complex non-linear analysis. We have:

$$
\mathbf{M} \ddot{\overline{\mathbf{x}}}+\mathbf{C} \dot{\overline{\mathbf{x}}}+\mathbf{K}_{\mathbf{t}} \overline{\mathbf{x}}=\mathbf{F}_{\text {friction }}^{\mathrm{NL}}(\overline{\mathbf{x}})
$$

where the complete expression of the non-linear friction vector $\mathbf{F}_{\text {friction }}(\overline{\mathbf{x}})$ is defined by

$$
\mathbf{F}_{\text {friction }}(\overline{\mathbf{x}})=\mathbf{F}_{\text {friction }}^{\mathbf{L}}(\overline{\mathbf{x}})+\mathbf{F}_{\text {friction }}^{\mathrm{NL}}(\overline{\mathbf{x}})
$$

$\mathbf{F}_{\text {friction }}^{\mathbf{L}}$ and $\mathbf{F}_{\text {friction }}^{\mathrm{NL}}$ contain the linear and non-linear expressions of $\mathbf{F}_{\text {friction }}$.

The terms $\mathbf{F}_{\text {friction }}^{\mathbf{L}}$ were defined previously in (218). The expression of the vector $\mathbf{F}_{\text {friction }}^{\mathrm{NL}}$ is given by

$$
\mathbf{F}_{\text {friction }}^{\mathbf{N L}}=\left\{\begin{array}{lllllllllllllll}
-F_{X}^{N L} & M_{Y}^{N L} & M_{Z}^{N L} & M_{X}^{N L} & F_{X}^{N L} & -M_{Y}^{N L} & -M_{Z}^{N L} & 0 & 0 & 0 & 0 & 0 & 0 & 0 & 0
\end{array}\right\}^{T}
$$

The analytical expressions of the non-linear terms $F_{X}^{N L}, M_{Y}^{N L}, M_{Z}^{N L}$ and $M_{X}^{N L}$ in terms of both equilibrium position $\mathbf{x}_{\mathbf{0}}$ and perturbation variables $\overline{\mathbf{x}}$ are given in Annexe $\mathrm{B}$.

$$
\mathbf{M} \ddot{\overline{\mathbf{x}}}+\mathbf{C} \dot{\overline{\mathbf{x}}}+\left(\mathbf{K}-\mathbf{K}_{\text {barre }}-\mathbf{K}_{\text {frottement }}^{\mathbf{L}}\right) \overline{\mathbf{x}}=\sum_{i=1}^{15} \sum_{j=1}^{15} \mathbf{f}_{(\mathbf{2})}^{\mathrm{ij}} \overline{x_{i} x_{j}}+\sum_{i=1}^{15} \sum_{j=1}^{15} \sum_{k=1}^{15} \mathbf{f}_{(\mathbf{3})}^{\mathbf{i j k}} \overline{x_{i} x_{j} x_{k}}
$$

where $\mathbf{f}_{(\mathbf{2})}^{\mathrm{ij}}$ and $\mathbf{f}_{(\mathbf{3})}^{\mathrm{ijk}}$ contain the quadratic and cubic non-linear terms. Next, the system is rewritten in state variables $\mathbf{y}=\left\{\begin{array}{ll}\mathbf{y} & \dot{\mathbf{y}}\end{array}\right\}^{T}$ in order to apply the extension of the centre manifold:

$$
\dot{\mathbf{y}}=\mathbf{A y}+\sum_{i=1}^{30} \sum_{j=1}^{30} \mathbf{g}_{(\mathbf{2})}^{\mathbf{i j}} y_{i} y_{j}+\sum_{i=1}^{30} \sum_{j=1}^{30} \sum_{k=1}^{30} \mathbf{g}_{(\mathbf{3})}^{\mathbf{i j k}} y_{i} y_{j} y_{k}
$$

where $\mathbf{A}$ is a $30 \times 30$ matrix. $\mathbf{g}_{(\mathbf{2})}^{\mathbf{i j}}$ and $\mathbf{g}_{(\mathbf{3})}^{\mathbf{i j k}}$ are 30 -dimensional vectors containing the quadratic and cubic non-linear terms.

As explained in the Section 3.4.2, the system may be reduced in the centre manifold variables near the Hopf bifurcation point as follows

$$
\left\{\begin{array}{l}
\dot{\mathbf{v}}_{\mathbf{c}}=\mathbf{J}_{\mathbf{c}}(\mu) \mathbf{v}_{\mathbf{c}}+\mathbf{G}_{\mathbf{2}}\left(\mathbf{v}_{\mathbf{c}}, \mathbf{h}\left(\mathbf{v}_{\mathbf{c}}\right), \mu\right)++\mathbf{G}_{\mathbf{3}}\left(\mathbf{v}_{\mathbf{c}}, \mathbf{h}\left(\mathbf{v}_{\mathbf{c}}\right), \mu\right) \\
\dot{\mu}=0 \\
\mu=\mu_{0}(1+\varepsilon) \quad(\varepsilon \ll 1) \\
\mathbf{v}_{\mathbf{s}}=\mathbf{h}\left(\mathbf{v}_{\mathbf{c}}\right)=\sum_{p=i+j=2}^{m} \sum_{j=0}^{p} \sum_{l=0}^{p} \mathbf{a}_{\mathbf{i j} 1} v_{c 1}^{i} v_{c 2}^{j} \bar{\mu}^{l} \quad(m \geq 2)
\end{array}\right.
$$


with $\mu_{0}$ the value of the friction coefficient at the Hopf bifurcation point. In this case, the centre manifold makes it possible to reduce the original non-linear system of 30 degree-of-freedom to only 3 variables $\mathbf{v}_{\mathbf{c}}=\left\{\begin{array}{lll}v_{c 1} & v_{c 2} & \mu\end{array}\right\}^{T}$. Next, the extension of the centre manifold approach via the fractional approximants is obtained. As explained in Section 3.4, the final reduced and simplified non-linear system is expressed as follows

$$
\left\{\begin{array}{l}
\dot{v_{c 1}}=[M, N]_{f_{1}\left(v_{c 1}, v_{c 2}\right)}=\sum_{i=0}^{3 m} \sum_{\substack{j=0 \\
1 \leq i+j \leq 3 m}}^{3 m} c_{1, i j} v_{c 1}^{i} v_{c 2}^{j} \quad(1 \leq k \leq 2) \\
\dot{v_{c 2}}=[M, N]_{f_{2}\left(v_{c 1}, v_{c 2}\right)}=\sum_{i=0}^{3 m} \sum_{\substack{j=0 \\
1 \leq i+j \leq 3 m}}^{3 m} c_{2, i j} v_{c 1}^{i} v_{c 2}^{j} \\
\dot{\mu}=0 \\
\mu=\mu_{0}(1+\varepsilon) \quad(\varepsilon \ll 1)
\end{array}\right.
$$

Finally, the non-linear solution of the system (226) is expressed as a truncated Fourier series:

$$
\left\{\begin{array}{l}
v_{c 1}(t)=V_{1,0}+\sum_{j=1}^{H}\left(V_{1,2 j-1} \cos (j \omega t)+V_{1,2 j} \sin (j \omega t)\right) \\
v_{c 2}(t)=V_{2,0}+\sum_{j=1}^{H}\left(V_{2,2 j-1} \cos (j \omega t)+V_{2,2 j} \sin (j \omega t)\right)
\end{array}\right.
$$

By applying the $3^{\text {th }}$-order centre manifold approach with the $[3 / 2]_{f}$ Symmetric-Off-Diagonal fractional rational approximants and the $2^{t h}$-order harmonic components $(H=2)$ for the Alternate/Frequency Time domain method, we reduce the number of equations of the original system from 30 to 2 and simplify the number of non-linear terms approximatively from 108000 to 28 , as indicated in Table 4 .

Then, the original complex non-linear system is reduced and simplified by retaining the essential non-linear dynamical behaviour of the original system as illustrated in Figures 56-63. The second-order polynomial approximation is not sufficient to provide a good approximation of the stable variables. This non-linear extension of the centre manifold approach via the rational approximants appears very interesting in regard to computational time and also requires very little computer resources, as indicated in Table 4.

\begin{tabular}{|c||c||c||c|}
\hline Methods & CPU time $(\mathrm{s})$ & Degree-of-freedom & Non-linear terms \\
\hline \hline Original system & $\approx 18000$ & 30 & $\approx 108000$ \\
\hline Reduced and simplified system & $\approx 20$ & 2 & 28 \\
\hline
\end{tabular}

Table 4: Comparison between the original and reduced system 


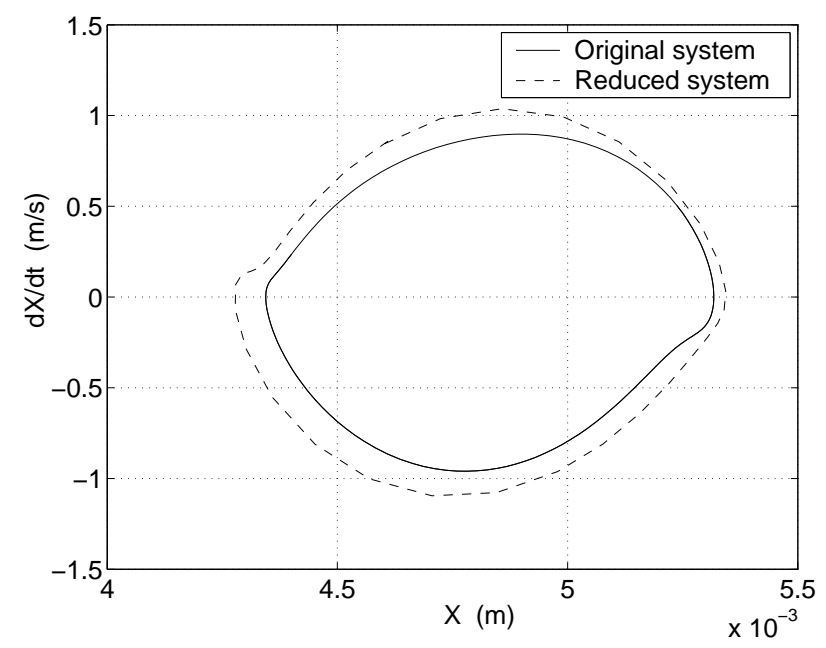

(a) Limit cycles $\left(x_{s}, \dot{x_{s}}\right)$

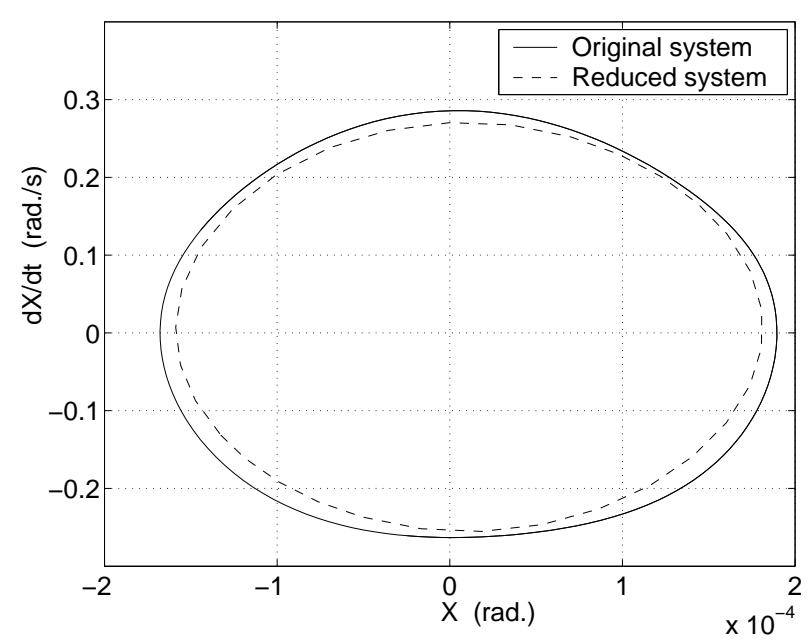

(b) Limit cycles $\left(\theta_{s}, \dot{\theta_{s}}\right)$

Figure 56: Limit cycles

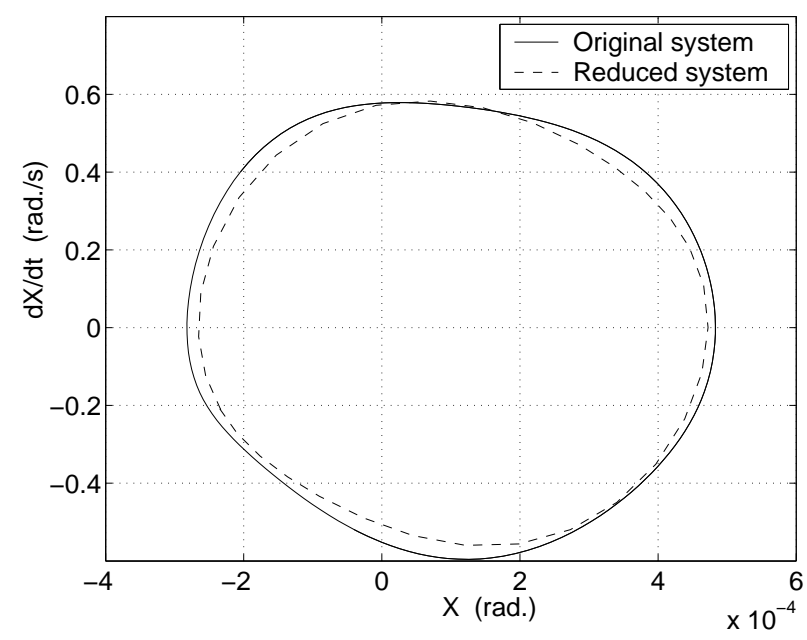

(a) Limit cycles $\left(\psi_{s}, \dot{\psi}_{s}\right)$

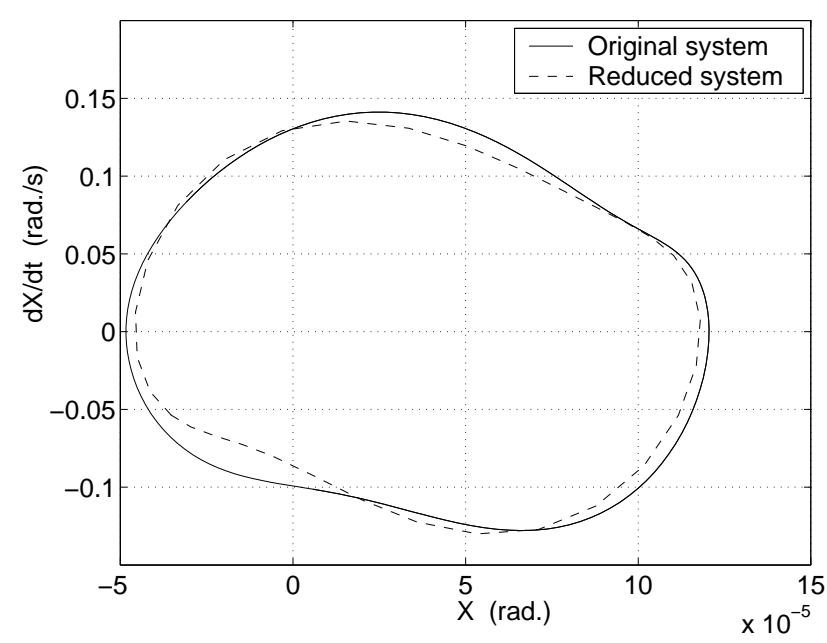

(b) Limit cycles $\left(\phi_{s}, \dot{\phi}_{s}\right)$

Figure 57: Limit cycles

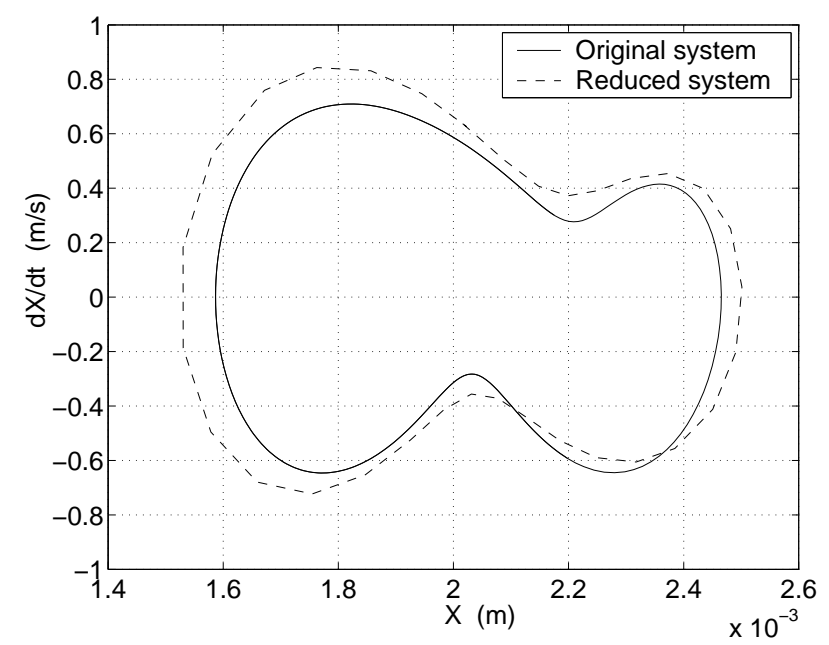

(a) Limit cycles $\left(x_{r}, \dot{x_{r}}\right)$

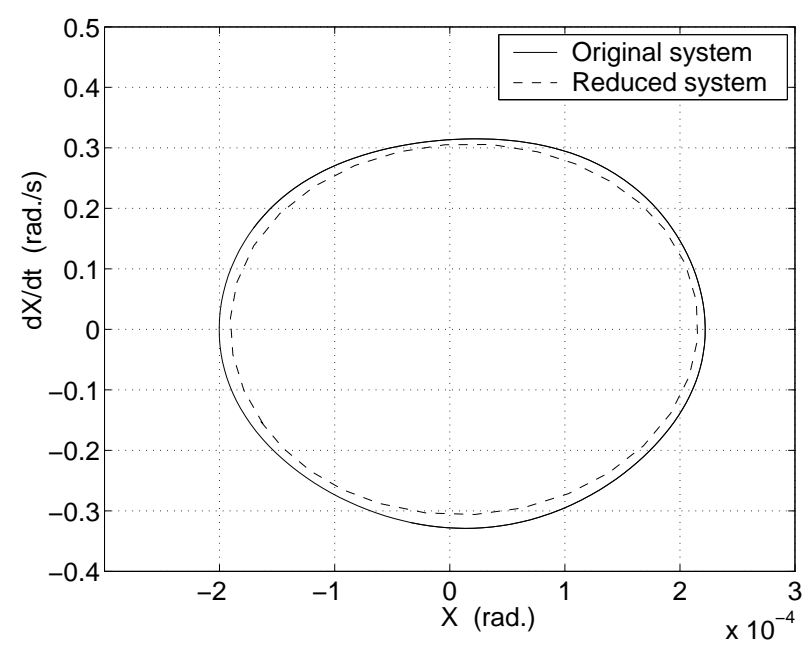

(b) Limit cycles $\left(\theta_{r}, \dot{\theta_{r}}\right)$

Figure 58: Limit cycles 


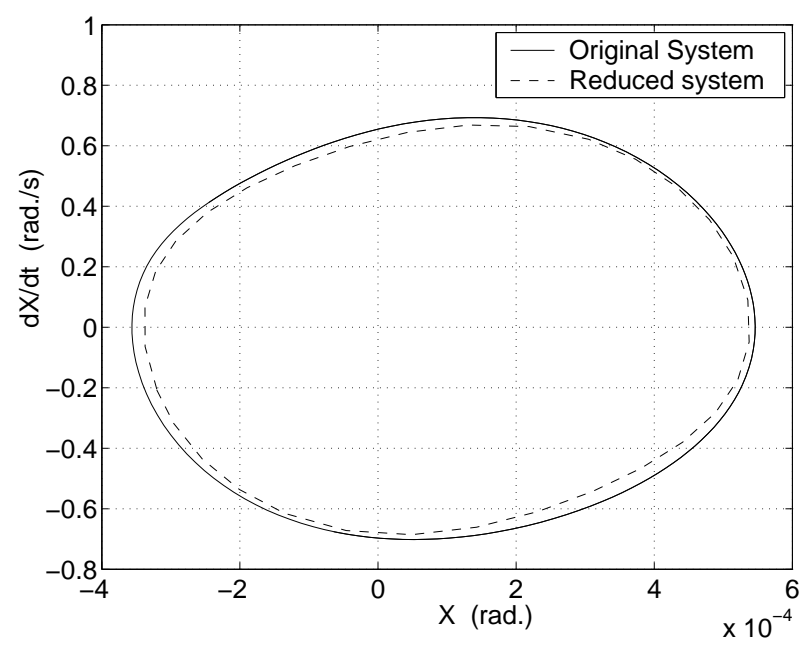

(a) Limit cycles $\left(\psi_{r}, \dot{\psi_{r}}\right)$

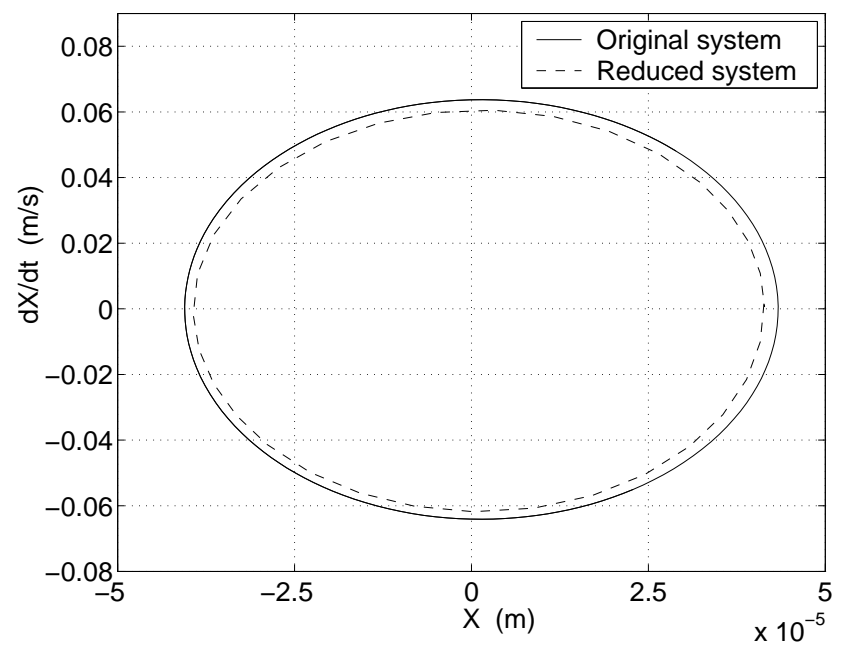

(b) Limit cycles $\left(y_{f}, \dot{y}_{f}\right)$

Figure 59: Limit cycles

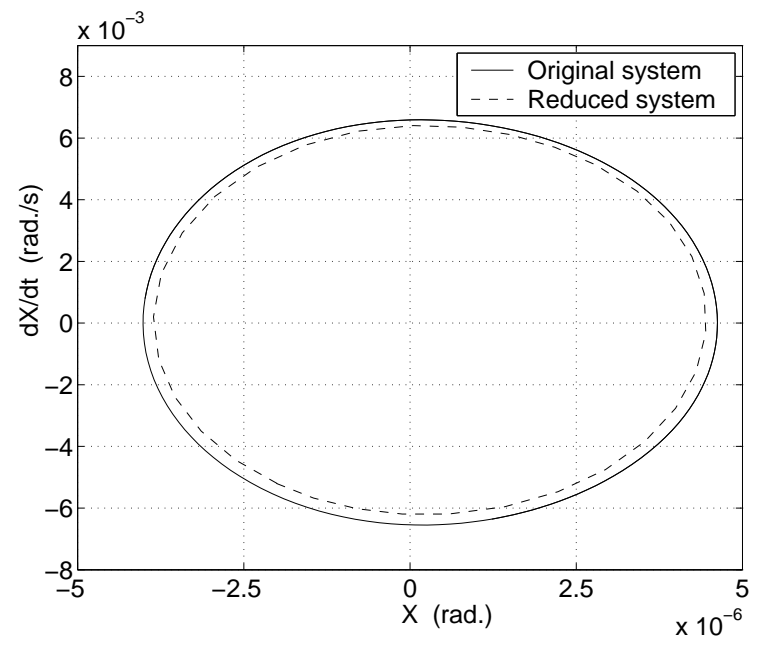

(a) Limit cycles $\left(\theta_{f}, \dot{\theta_{f}}\right)$

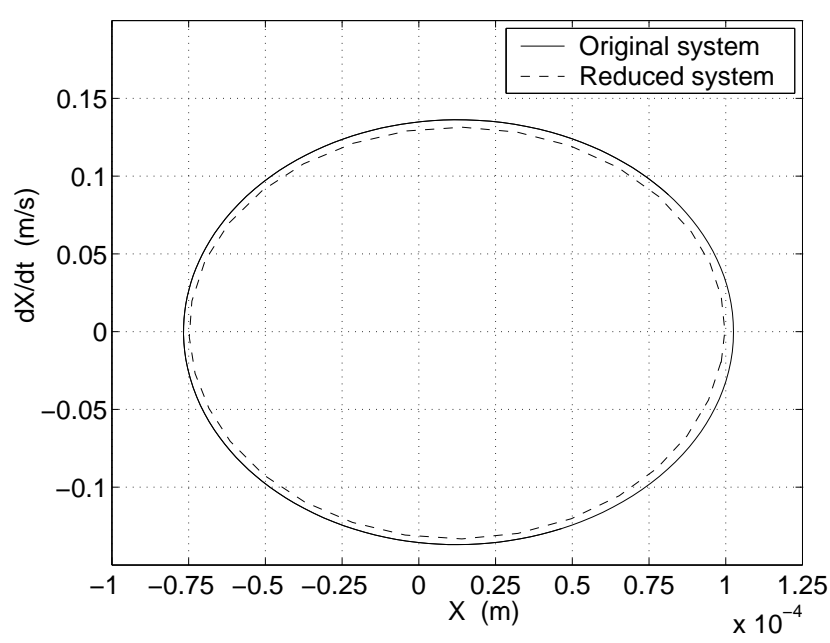

(b) Limit cycles $\left(z_{f}, \dot{z_{f}}\right)$

Figure 60: Limit cycles

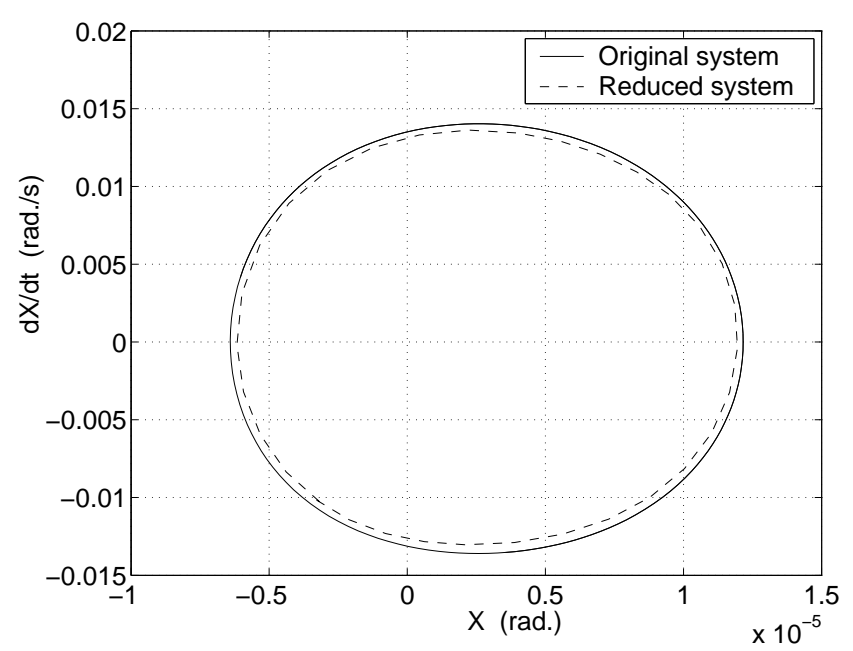

(a) Limit cycles $\left(\psi_{f}, \dot{\psi_{f}}\right)$

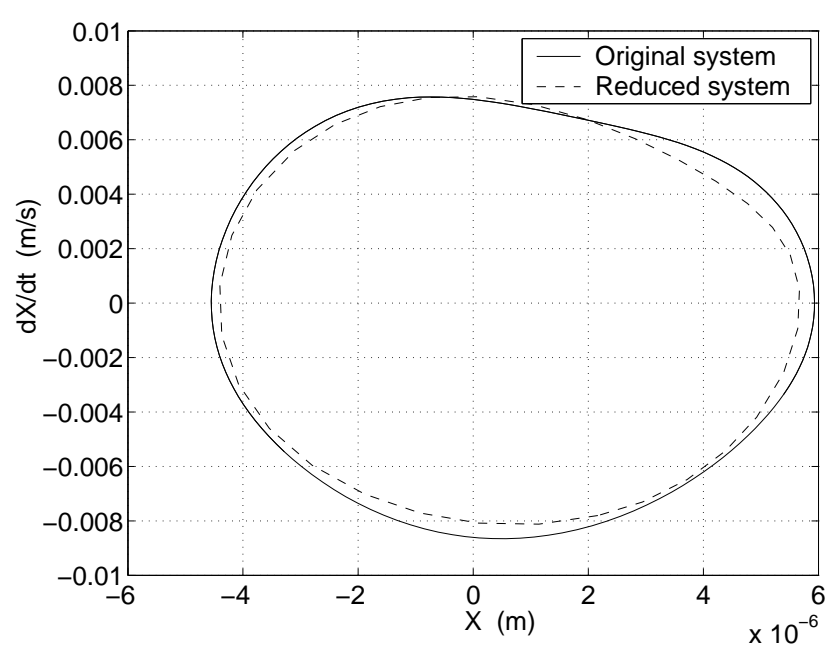

(b) Limit cycles $\left(y_{t}, \dot{y_{t}}\right)$

Figure 61: Limit cycles 


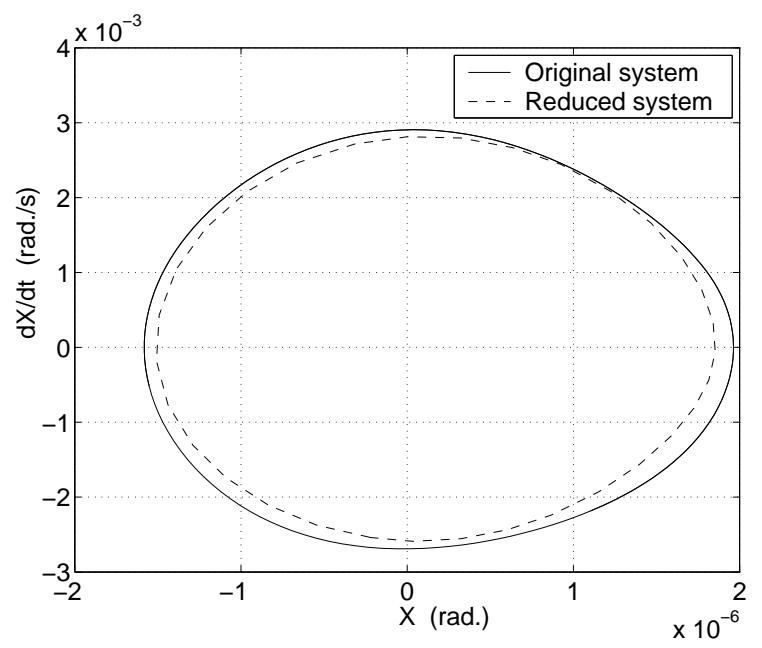

(a) Limit cycles $\left(\theta_{t}, \dot{\theta_{t}}\right)$

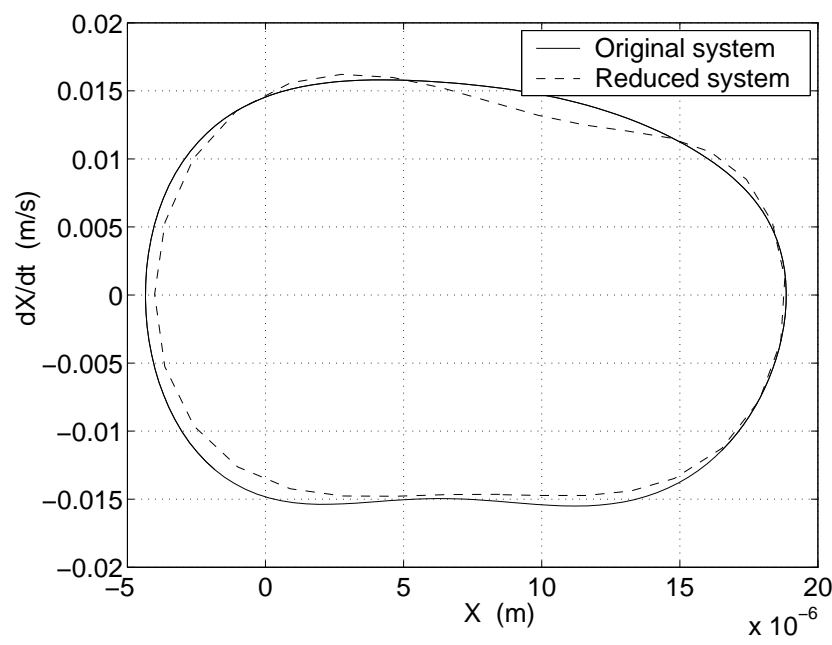

(b) Limit cycles $\left(z_{t}, \dot{z}_{t}\right)$

Figure 62: Limit cycles

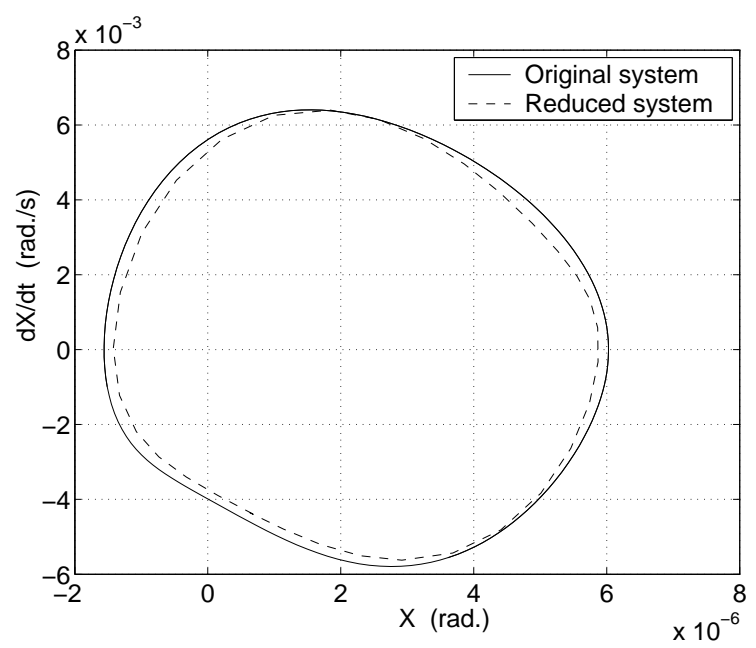

Figure 63: Limit cycles $\left(\psi_{t}, \dot{\psi_{t}}\right)$

\section{Conclusion and future research directions}

In this paper, non-linear methods to reduce the non-linear mechanical systems for instability computation have been developed. The centre manifold, the rational approximants and an harmonic balance method, called the Alternate Frequency/Time domain method, have been introduced; all the associated computational techniques have been discussed in detail. The results from these non-linear approaches have been compared with those obtained by integrating the full original system. Excellent agreement was found between the original and the reduced system. The centre manifold theory and the rational approximants allow us to reduce the number of equations of the original system and to simplify the non-linear terms in order to obtain a simplified system, without losing the dynamics of the original system, as well as the contributions of the non-linear terms. The harmonic balance method makes it possible to find the non-linear response of the reduced and simplified system as an assumed truncated Fourier series.

One of the main purposes of this paper is to present a new non-linear procedure consisting of applying the rational fractional approximants after the centre manifold method. This procedure appears very interesting in regard to computation time and it requires less computer resources due to the number of stable coefficients used to obtained the limit cycle amplitude. The powerful property used in this study is indeed that a sequence of rational fractional approximants may converge even if the associated series does not; we can than extended our domain of convergence. Moreover, the domain of validity of the solution is successfully enhanced by employing rational fractional approx- 
imants in this study. The rational fractional approximants show superior performance over series approximations. The computational techniques for the three non-linear methods have been tested for two examples: the first example was a two degree-of-freedom system with quadratic and cubic non-linearities. It was developed to demonstrate and test all the advantages and disadvantages of each method. The second example was a complex system with many degrees-of-freedom and polynomial non-linearities. The suitability and capacity of the new non-linear technique using the centre manifold and the rational approximants was clearly demonstrated. Moreover, this non-linear procedure method requires less computer resources and appears to be particularly interesting in cases of large non-linear systems.

One of the difficulties when the centre manifold or the rational approximants are applied is the determination of the order of the polynomial approximation of the stable variables in a power series in centre variable, and the determination of the order of the numerator and denominator for the centre manifold approach and the rational approximants, respectively. Usually, high orders become less significant when they increase, and hence we may find the necessary and significant order to obtain a good approximation of the non-linear dynamical system; in the field of mechanical engineering, the choice of the orders for the centre manifold approach and for the rational approximants are selected to retain only the significant order by iterative approaches. It should be very interesting to find and to implement systematic computational procedures to determine the lowest order needed to obtain a good approximation of the non-linear dynamical system.

Even if this non-linear technique appears very interesting in regard to reduction and simplification of mechanical systems subject to instability phenomena, it has the disadvantage of being applicable only to systems with polynomial non-linearities. Moreover, the centre manifold approach and the associated theorem [116] characterize the local bifurcation analysis near a fixed point of the non-linear system. This assumption reduced the fields of application and it should be interesting to estimate the non-linear dynamical response of mechanical systems far from the Hopf bifurcation point. Also, new methods combining various approaches including at the same time the notion of reduction via the centre manifold approach and a modal analysis approach based on the non-linear modes should be very interesting [176].

Moreover, the extension of non-linear methods that reduce the dimension of mechanical systems would offer powerful techniques for non-linear systems with various non-linearities such as discontinuous phenomena: in the field of mechanical engineering, various applications are concerned with this type of non-linear problem that may cause a great deal of instability phenomena. Because non-linear dynamical structures depending on control parameters are encountered in many areas of science and engineering, a systematic computational implementation in finite elements software for non-linear methods such as the centre manifold approach or the extension proposed in this paper that reduce the dimension of non-linear systems would be a powerful tool. This computational and systematic treatment in finite element software is perhaps a difficult and ambitious task but not unrealistic.

\section{Annexe A: Analytical expression for the center manifold coefficients}

We note $J_{c 1}$ et $J_{c 2}$, the first and second terms of the matrix $\mathbf{J}_{\mathbf{c}}$, respectively; $J_{s k}$ the $k^{\text {th }}$-diagonal term of the matrix $\mathbf{J}_{\mathbf{s}}$; and $A^{i, j}$ the $i^{t h}$-ligne $j^{\text {th }}$-column of the matrix $\mathbf{A}$.

\section{Second order analytical expression}

The stable variables $\mathbf{v}_{\mathbf{s}}$ are approximated as a power series of order two in the center variables $\left(\mathbf{v}_{\mathbf{c}}, \hat{\mu}\right)$ :

$$
\begin{aligned}
\mathbf{v}_{\mathbf{S}} & =\mathbf{h}^{(\mathbf{1})}\left(\mathbf{v}_{\mathbf{c}}, \hat{\mu}\right)=\sum_{p=i+j+l=2}^{2} \sum_{j=0}^{p} \sum_{l=0}^{p} \mathbf{a}_{\mathbf{i j 1}} v_{c 1}^{i} v_{c 2}^{j} \hat{\mu}^{l} \\
& =\mathbf{a}_{\mathbf{2 0 0}} v_{c 1}^{2}+\mathbf{a}_{\mathbf{1 1 0}} v_{c 1} v_{c 2}+\mathbf{a}_{\mathbf{0 2 0}} v_{c 2}^{2}+\mathbf{a}_{\mathbf{1 0 1}} v_{c 1} \hat{\mu}+\mathbf{a}_{\mathbf{0 1 1}} v_{c 2} \hat{\mu}+\mathbf{a}_{\mathbf{0 0 2}} \hat{\mu}^{2}
\end{aligned}
$$

where $\mathbf{a}_{200}, \mathbf{a}_{110}, \mathbf{a}_{020}, \mathbf{a}_{101}, \mathbf{a}_{011}$ and $\mathbf{a}_{002}$ are the unknown center manifold coefficients of order two. The analytical expressions of the center manifold coefficients for the $k^{t h}$-stable variable are given by

$$
a_{k, 200}=\frac{H_{(2)}^{k, 1}}{2 J_{c 1}-J_{s k}}
$$




$$
\begin{gathered}
a_{k, 110}=\frac{H_{(2)}^{k, 2}+H_{(2)}^{k, n+2}}{J_{c 1}+J_{c 2}-J_{s k}} \\
a_{k, 020}=\frac{H_{(2)}^{k, n+3}}{2 J_{c 2}-J_{s k}} \\
a_{k, 101}=\frac{H_{(2)}^{k, n+1}+H_{(2)}^{k, n(n+1)+1}}{J_{c 1}-J_{s k}} \\
a_{k, 011}=\frac{H_{(2)}^{k, 2(n+1)}+H_{(2)}^{k, n(n+1)+2}}{J_{c 2}-J_{s k}} \\
a_{k, 002}=\frac{-H_{(2)}^{k,(n+1)^{2}}}{J_{s k}}
\end{gathered}
$$

\section{Third order analytical expression}

The stable variables $\mathbf{v}_{\mathbf{s}}$ are approximated as a power series of order three in the center variables $\left(\mathbf{v}_{\mathbf{c}}, \hat{\mu}\right)$ :

$$
\mathbf{v}_{\mathbf{s}}=\mathbf{h}\left(\mathbf{v}_{\mathbf{c}}, \hat{\mu}\right)=\sum_{p=i+j+l=2}^{3} \sum_{j=0}^{p} \sum_{l=0}^{p} \mathbf{a}_{\mathbf{i j} \mathbf{1}} v_{c 1}^{i} v_{c 2}^{j} \hat{\mu}^{l}
$$

So, we have

$$
\begin{aligned}
\mathbf{v}_{\mathbf{s}}= & \mathbf{h}^{(\mathbf{2})}\left(\mathbf{v}_{\mathbf{c}}, \hat{\mu}\right)=\mathbf{h}^{(\mathbf{1})}\left(\mathbf{v}_{\mathbf{c}}, \hat{\mu}\right)+\mathbf{a}_{\mathbf{3 0 0}} v_{c 1}^{3}+\mathbf{a}_{\mathbf{2 1 0}} v_{c 1}^{2} v_{c 2}+\mathbf{a}_{\mathbf{1 2 0}} v_{c 1} v_{c 2}^{2}+\mathbf{a}_{\mathbf{0 3 0}} v_{c 2}^{2} \\
& +\mathbf{a}_{\mathbf{2 0 1}} v_{c 1}^{2} \hat{\mu}+\mathbf{a}_{\mathbf{1 1 1}} v_{c 1} v_{c 2} \hat{\mu}+\mathbf{a}_{\mathbf{0 2 1}} v_{c 2}^{2} \hat{\mu}+\mathbf{a}_{\mathbf{1 0 2}} v_{c 1} \hat{\mu}^{2}+\mathbf{a}_{\mathbf{0 1 2}} v_{c 2} \hat{\mu}^{2}+\mathbf{a}_{\mathbf{0 0 3}} \hat{\mu}^{3}
\end{aligned}
$$

where $\mathbf{a}_{300}, \mathbf{a}_{210}, \mathbf{a}_{120}, \mathbf{a}_{030}, \mathbf{a}_{201}, \mathbf{a}_{111}, \mathbf{a}_{021}, \mathbf{a}_{102}, \mathbf{a}_{012}$ and $\mathbf{a}_{003}$ are the unknown center manifold coefficients of order three.

The analytical expressions of the center manifold coefficients for the $k^{\text {th }}$-stable variable are given by

$$
\begin{gathered}
a_{k, 300}=\frac{-2 a_{k, 200} G_{(2)}^{1,1}-a_{k, 110} G_{(2)}^{2,1}+H_{(3)}^{k 1}+\sum_{i=1}^{n-2} a_{i, 200}\left(H_{(2)}^{k, 2+i}+H_{(2)}^{k,(n+1)(i+1)+1}\right)}{3 J_{c 1}-J_{s k}} \\
-2 a_{k, 200}\left(G_{(2)}^{1,2}+G_{(2)}^{1, n+2}\right)-a_{k, 110}\left(G_{(2)}^{1,1}+G_{(2)}^{2,2}+G_{(2)}^{2, n+2}\right)-2 a_{k, 020} G_{(2)}^{21}+H_{(3)}^{k, 2}+H_{(3)}^{k, n+2} \\
+a_{(3)}^{k,(n+1)^{2}+1}+\sum_{i=1}^{n-2} a_{i, 110}\left(H_{(2)}^{k, i+2}+H_{(2)}^{k,(n+1)(i+1)+1}\right)+\sum_{i=1}^{n-2} a_{i, 200}\left(H_{(2)}^{k, n+i+3}+H_{(2)}^{k,(n+1)(i+1)+2}\right) \\
2=\frac{-2 J_{c 1}+J_{c 2}-J_{s k}}{-2 a_{k, 020}\left(G_{(2)}^{2,2}+G_{(2)}^{2, n+2}\right)-a_{k, 110}\left(G_{(2)}^{2, n+3}+G_{(2)}^{1, n+3}+H_{(3)}^{k, n+3}+H_{(3)}^{k,(n+1)^{2}+2}+H_{(2)}^{k,(n+1)^{2}+n+2}\right)} \\
+\sum_{i=1}^{n-2} a_{i, 020}\left(H_{(2)}^{k, i+2}+H_{(2)}^{k,(n+1)(i+1)+1}\right)+\sum_{i=1}^{n-2} a_{i, 110}\left(H_{(2)}^{k, n+i+3}+H_{(2)}^{k,(n+1)(i+1)+2}\right) \\
a_{k, 120}=\frac{J_{c 1}+2 J_{c 2}-J_{s k}}{(239)}
\end{gathered}
$$




$$
\begin{gathered}
a_{k, 030}=\frac{-2 a_{k, 020} G_{(2)}^{2, n+3}-a_{k, 110} G_{(2)}^{1, n+3}+H_{(3)}^{k,(n+1)^{2}+n+3}+\sum_{i=1}^{n-2} a_{i, 020}\left(H_{(2)}^{k, n+i+3}+H_{(2)}^{k,(n+1)(i+1)+2}\right)}{3 J_{c 2}-J_{s k}} \\
-a_{k, 101} G_{(2)}^{1,1}-a_{k, 011} G_{(2)}^{2,1}-2 a_{k, 200}\left(G_{(2)}^{1, n+1}+G_{(2)}^{1, n(n+1)+1}\right)-a_{k, 110}\left(G_{(2)}^{2, n+1}+G_{(2)}^{2, n(n+1)+1}\right) \\
+H_{(3)}^{k, n(n+1)^{2}+1}+H_{(3)}^{k, n(n+1)+1}+H_{(3)}^{k, n+1} \\
a_{k, 201}=\frac{+\sum_{i=1}^{n-2} a_{i, 101}\left(H_{(2)}^{k, i+2}+H_{(2)}^{k,(n+1)(i+1)+1}\right)+\sum_{i=1}^{n-2} a_{i, 200}\left(H_{(2)}^{k,(n+1)(2+i)}+H_{(2)}^{k,(n+1) n+i+2}\right)}{2 J_{c 1}-J_{s k}}
\end{gathered}
$$

$$
\begin{gathered}
-a_{k, 101} G_{(2)}^{1, n+3}-a_{k, 011} G_{(2)}^{2, n+3}-2 a_{k, 020}\left(G_{(2)}^{2,2(n+1)}+G_{(2)}^{2,(n+1) n+2}\right)-a_{k, 110}\left(G_{(2)}^{1,2(n+1)}+G_{(2)}^{1,(n+1) n+2}\right) \\
+H_{(3)}^{k,(n+1)^{2}+2(n+1)}+H_{(3)}^{k, 2(n+1)^{2}-n+1}+H_{(3)}^{k,(n+1)^{3}-2(2 n+1)}
\end{gathered}
$$$$
a_{k, 021}=\frac{+\sum_{i=1}^{n-2} a_{i, 020}\left(H_{(2)}^{k,(n+1)(2+i)}+H_{(2)}^{k,(n+1) n+i+2}\right)+\sum_{i=1}^{n-2} a_{i, 011}\left(H_{(2)}^{k, 2 n-1+i}+H_{(2)}^{k,(n+1)(i+1)+2}\right)}{2 J_{c 2}-J_{s k}}
$$

$$
\begin{aligned}
& -2 a_{k, 200} G_{(2)}^{1,(n+1)^{2}}-a_{k, 110} G_{(2)}^{2,(n+1)^{2}}-a_{k, 101}\left(G_{(2)}^{1, n+1}+G_{(2)}^{1,(n+1) n+1}\right) \\
& -a_{k, 011}\left(G_{(2)}^{2, n+1}+G_{(2)}^{2,(n+1) n+1}\right)+H_{(3)}^{k,(n+1)^{2}}+H_{(3)}^{k, n(n+1)^{2}+n+1}+H_{(3)}^{k,(n+1)^{3}-n} \\
& \frac{+\sum_{i=1}^{n-2} a_{i, 101}\left(H_{(2)}^{k,(n+1)(2+i)}+H_{(2)}^{k,(n+1) n+i+2}\right)+\sum_{i=1}^{n-2} a_{i, 002}\left(H_{(2)}^{k, i+2}+H_{(2)}^{k,(n+1)(i+1)+1}\right)}{J_{c 1}-J_{s k}} \\
& -a_{k, 110} G_{(2)}^{1,(n+1)^{2}}-2 a_{k, 020} G_{(2)}^{2,(n+1)^{2}}-a_{k, 101}\left(G_{(2)}^{1,2(n+1)}+G_{(2)}^{1, n(n+1)+2}\right) \\
& -a_{k, 011}\left(G_{(2)}^{2,2(n+1)}+G_{(2)}^{2, n(n+1)+2}\right)+H_{(3)}^{k, 2(n+1)^{2}}+H_{(3)}^{k, n(n+1)^{2}+2(n+1)}+H_{(3)}^{k,(n+1)^{3}-n+1} \\
& a_{k, 012}=\frac{+\sum_{i=1}^{n-2} a_{i, 011}\left(H_{(2)}^{k,(n+1)(2+i)}+H_{(2)}^{k,(n+1) n+i+2}\right)+\sum_{i=1}^{n-2} a_{i, 002}\left(H_{(2)}^{k, 2 n-1+i}+H_{(2)}^{k,(n+1)(i+1)+2}\right)}{J_{c 2}-J_{s k}} \\
& -a_{k, 101}\left(G_{(2)}^{12}+G_{(2)}^{1, n+2}\right)-a_{k, 110}\left(G_{(2)}^{1, n+1}+G_{(2)}^{1, n(n+1)+1}+G_{(2)}^{1,2(n+1)}+G_{(2)}^{1, n(n+1)+2}\right) \\
& -2 a_{k, 200}\left(G_{(2)}^{1,2(n+1)}+G_{(2)}^{1, n(n+1)+2}\right)-2 a_{k, 020}\left(G_{(2)}^{2, n+1}+G_{(2)}^{2, n(n+1)+1}\right)-a_{k, 011}\left(G_{(2)}^{22}+G_{(2)}^{2, n+2}\right) \\
& +H_{(3)}^{k, 2(n+1)}+H_{(3)}^{k, n(n+1)+2}+H_{(3)}^{k,(n+1)^{2}+n+1}+H_{(3)}^{k, 2(n+1)^{2}-n}+H_{(3)}^{k, n(n+1)^{2}+n-2} \\
& +H_{(3)}^{k, n(n+1)^{2}+n+2}+\sum_{i=1}^{n-2} a_{i, 110}\left(H_{(2)}^{k,(n+1)(2+i)}+H_{(2)}^{k,(n+1) n+i+2}\right) \\
& +\sum_{i=1}^{n-2} a_{i, 011}\left(H_{(2)}^{k, i+2}+H_{(2)}^{k,(n+1)(i+1)+1}\right)+\sum_{i=1}^{n-2} a_{i, 101}\left(H_{(2)}^{k, 2 n-1+i}+H_{(2)}^{k,(n+1)(i+1)+2}\right) \\
& a_{k, 111}=\frac{\sum_{i=1}}{J_{c 1}+J_{c 2}-J_{s k}}
\end{aligned}
$$




\section{Annexe B: linear and non-linear expressions of $F_{X}, M_{X}, M_{Y}$ and $M_{Z}$}

The linear expressions $F_{X}^{L}, M_{X}^{L}, M_{Y}^{L}$ and $M_{Z}^{L}$ of $F_{X}, M_{X}, M_{Y}$ and $M_{Z}$ are given by

$$
\begin{array}{rl}
F_{X}^{L}(\overline{\mathbf{x}})= & \left(K_{1} A_{2}+2 K_{2} A_{2} B_{x}+3 K_{3} A_{2} B_{x}^{2}+\frac{3}{4} K_{3} A_{4} B_{\theta}^{2}+\frac{3}{4} K_{3} A_{4} B_{\psi}^{2}\right)\left(\overline{x_{s}}-\overline{x_{r}}\right) \\
& +\left(\frac{1}{2} K_{2} A_{4} B_{\psi}+\frac{3}{2} K_{3} A_{4} B_{\psi} B_{\theta}\right)\left(\overline{\psi_{s}}-\overline{\psi_{r}}\right)+\left(\frac{1}{2} K_{2} A_{4} B_{\theta}+\frac{3}{2} K_{3} A_{4} B_{\psi} B_{\theta}\right)\left(\overline{\theta_{s}}-\overline{\theta_{r}}\right) \\
M_{X}^{L}(\overline{\mathbf{x}})=2 & N \mu_{b r k}\left(\left(\frac{2}{3} K_{1} A_{3}+\frac{4}{3} K_{2} A_{3} B_{x}+2 K_{3} A_{3} B_{x}^{2}+\frac{3}{5} K_{3} A_{5} B_{\theta}^{2}+\frac{3}{5} K_{3} A_{5} B_{\psi}^{2}\right)\left(\overline{x_{s}}-\overline{x_{r}}\right)\right. \\
+ & \left.\left(\frac{2}{5} K_{2} A_{5} B_{\theta}+\frac{6}{5} K_{3} A_{5} B_{x} B_{\theta}\right)\left(\overline{\theta_{s}}-\overline{\theta_{r}}\right)+\left(\frac{2}{5} K_{2} A_{5} B_{\psi}+\frac{6}{5} K_{3} A_{5} B_{x} B_{\psi}\right)\left(\overline{\psi_{s}}-\overline{\psi_{r}}\right)\right) \\
M_{Y}^{L}(\overline{\mathbf{x}})= & \left(-\frac{1}{2} K_{2} A_{4} B_{\theta}+\frac{3}{2} K_{3} A_{4} B_{x} B_{\theta}\right)\left(\overline{x_{s}}-\overline{x_{r}}\right)+\left(-\frac{1}{4} K_{3} A_{6} B_{\theta} B_{\psi}\right)\left(\overline{\psi_{s}}-\overline{\psi_{r}}\right) \\
& +\left(-\frac{1}{4} K_{1} A_{4}-\frac{1}{2} K_{2} A_{4} B_{x}-\frac{3}{4} K_{3} A_{4} B_{x}^{2}-\frac{1}{7} K_{3} A_{6} B_{\psi}^{2}-\frac{3}{8} K_{3} A_{6} B_{\theta}^{2}\right)\left(\overline{\theta_{s}}-\overline{\theta_{r}}\right) \\
M_{Z}^{L}(\overline{\mathbf{x}})= & \left(-\frac{1}{2} K_{2} A_{4} B_{\psi}+\frac{3}{2} K_{3} A_{4} B_{x} B_{\psi}\right)\left(\overline{x_{s}}-\overline{x_{r}}\right)+\left(-\frac{1}{4} K_{3} A_{6} B_{\theta} B_{\psi}\right)\left(\overline{\theta_{s}}-\overline{\theta_{r}}\right) \\
& +\left(-\frac{1}{4} K_{1} A_{4}-\frac{1}{2} K_{2} A_{4} B_{x}-\frac{3}{4} K_{3} A_{4} B_{x}^{2}-\frac{1}{7} K_{3} A_{6} B_{\theta}^{2}-\frac{3}{8} K_{3} A_{6} B_{\psi}^{2}\right)\left(\overline{\psi_{s}}-\overline{\psi_{r}}\right)
\end{array}
$$

with $B_{x}=x_{s 0}-x_{r 0}, B_{\psi}=\psi_{s 0}-\psi_{r 0}, B_{\theta}=\theta_{s 0}-\theta_{r 0}$, and $A_{k}=\pi\left(R_{e}^{k}-R_{i}^{k}\right)$ for $k=1,2,3,4, \ldots, 6$.

The non-linear expressions $F_{X}^{N L}, M_{Y}^{N L}$ and $M_{Z}^{N L}$ of $F_{X}, M_{X}, M_{Y}$ and $M_{Z}$ are given by

$$
\begin{aligned}
& F_{X}^{N L}(\bar{x})=\left(K_{2} A_{2}+3 K_{3} A_{2} B_{x}\right)\left(\overline{x_{s}}-\overline{x_{r}}\right)^{2}+\left(\frac{1}{4} K_{2} A_{4}+\frac{3}{4} K_{3} A_{4} B_{x}\right)\left(\overline{\theta_{s}}-\overline{\theta_{r}}\right)^{2} \\
& +\left(\frac{1}{4} K_{2} A_{4}+\frac{3}{4} K_{3} A_{4} B_{x}\right)\left(\overline{\psi_{s}}-\overline{\psi_{r}}\right)^{2}+\frac{3}{2} K_{3} A_{4} B_{\theta}\left(\overline{\theta_{s}}-\overline{\theta_{r}}\right)\left(\overline{x_{s}}-\overline{x_{r}}\right) \\
& +\frac{3}{2} K_{3} A_{4} B_{\psi}\left(\overline{\psi_{s}}-\overline{\psi_{r}}\right)\left(\overline{x_{s}}-\overline{x_{r}}\right)+K_{3} A_{2}\left(\overline{x_{s}}-\overline{x_{r}}\right)^{3}+\frac{3}{4} K_{3} A_{4}\left(\overline{\theta_{s}}-\overline{\theta_{r}}\right)^{2}\left(\overline{x_{s}}-\overline{x_{r}}\right) \\
& +\frac{3}{4} K_{3} A_{4}\left(\overline{\psi_{s}}-\overline{\psi_{r}}\right)^{2}\left(\overline{x_{s}}-\overline{x_{r}}\right) \\
& M_{X}^{N L}(\bar{x})=2 N \mu_{b r k}\left(\left(\frac{2}{3} K_{2} A_{3}+2 K_{3} A_{3} B_{x}\right)\left(\overline{x_{s}}-\overline{x_{r}}\right)^{2}+\left(\frac{1}{5} K_{2} A_{5}+\frac{3}{5} K_{3} A_{5} B_{x}\right)\left(\overline{\theta_{s}}-\overline{\theta_{r}}\right)^{2}\right. \\
& +\left(\frac{1}{5} K_{2} A_{5}+\frac{3}{5} K_{3} A_{5} B_{x}\right)\left(\overline{\psi_{s}}-\overline{\psi_{r}}\right)^{2}+\frac{6}{5} K_{3} A_{5} B_{\theta}\left(\overline{\theta_{s}}-\overline{\theta_{r}}\right)\left(\overline{x_{s}}-\overline{x_{r}}\right) \\
& +\frac{6}{5} K_{3} A_{5} B_{\psi}\left(\overline{\psi_{s}}-\overline{\psi_{r}}\right)\left(\overline{x_{s}}-\overline{x_{r}}\right)+\frac{2}{3} K_{3} A_{3}\left(\overline{x_{s}}-\overline{x_{r}}\right)^{3} \\
& \left.+\frac{3}{5} K_{3} A_{5}\left(\overline{\theta_{s}}-\overline{\theta_{r}}\right)^{2}\left(\overline{x_{s}}-\overline{x_{r}}\right)+\frac{3}{5} K_{3} A_{5}\left(\overline{\psi_{s}}-\overline{\psi_{r}}\right)^{2}\left(\overline{x_{s}}-\overline{x_{r}}\right)\right) \\
& M_{Y}^{N L}(\bar{x})=-\frac{3}{4} K_{3} A_{4} B_{\theta}\left(\overline{x_{s}}-\overline{x_{r}}\right)^{2}-\frac{3}{8} K_{3} A_{6} B_{\theta}\left(\overline{\theta_{s}}-\overline{\theta_{r}}\right)^{2}-\frac{1}{8} K_{3} A_{6} B_{\theta}\left(\overline{\psi_{s}}-\overline{\psi_{r}}\right)^{2} \\
& -\left(\frac{1}{2} K_{2} A_{4}+\frac{3}{2} K_{3} A_{4} B_{x}\right)\left(\overline{\theta_{s}}-\overline{\theta_{r}}\right)\left(\overline{x_{s}}-\overline{x_{r}}\right)-\frac{3}{8} K_{3} A_{6} B_{\psi}\left(\overline{\psi_{s}}-\overline{\psi_{r}}\right)\left(\overline{\theta_{s}}-\overline{\theta_{r}}\right) \\
& +\frac{1}{8} K_{3} A_{6}\left(\overline{\theta_{s}}-\overline{\theta_{r}}\right)^{3}+\frac{3}{4} K_{3} A_{4}\left(\overline{\theta_{s}}-\overline{\theta_{r}}\right)\left(\overline{x_{s}}-\overline{x_{r}}\right)^{2}+\frac{1}{8} K_{3} A_{6}\left(\overline{\theta_{s}}-\overline{\theta_{r}}\right)\left(\overline{\psi_{s}}-\overline{\psi_{r}}\right)^{2} \\
& M_{Z}^{N L}(\bar{x})=-\frac{3}{4} K_{3} A_{4} B_{\psi}\left(\overline{x_{s}}-\overline{x_{r}}\right)^{2}-\frac{3}{8} K_{3} A_{6} B_{\psi}\left(\overline{\psi_{s}}-\overline{\psi_{r}}\right)^{2}-\frac{1}{8} K_{3} A_{6} B_{\psi}\left(\overline{\theta_{s}}-\overline{\theta_{r}}\right)^{2} \\
& -\left(\frac{1}{2} K_{2} A_{4}+\frac{3}{2} K_{3} A_{4} B_{x}\right)\left(\overline{\psi_{s}}-\overline{\psi_{r}}\right)\left(\overline{x_{s}}-\overline{x_{r}}\right)-\frac{3}{8} K_{3} A_{6} B_{\psi}\left(\overline{\theta_{s}}-\overline{\theta_{r}}\right)\left(\overline{\psi_{s}}-\overline{\psi_{r}}\right) \\
& +\frac{1}{8} K_{3} A_{6}\left(\overline{\psi_{s}}-\overline{\psi_{r}}\right)^{3}+\frac{3}{4} K_{3} A_{4}\left(\overline{\psi_{s}}-\overline{\psi_{r}}\right)\left(\overline{x_{s}}-\overline{x_{r}}\right)^{2}+\frac{1}{8} K_{3} A_{6}\left(\overline{\psi_{s}}-\overline{\psi_{r}}\right)\left(\overline{\theta_{s}}-\overline{\theta_{r}}\right)^{2}
\end{aligned}
$$

with $B_{x}=x_{s 0}-x_{r 0}, B_{\psi}=\psi_{s 0}-\psi_{r 0}, B_{\theta}=\theta_{s 0}-\theta_{r 0}$ and $A_{k}=\pi\left(R_{e}^{k}-R_{i}^{k}\right)$ for $k=1,2,3,4, \ldots, 6$. 


\section{Nomenclature}

$\begin{array}{ll}x & \text { scalaire } \\ \mathbf{x} & \text { vector of displacement } \\ \dot{\mathbf{x}} & \text { vector of velocity } \\ \ddot{\mathbf{x}} & \text { vector of acceleration } \\ \mathbf{x}_{\mathbf{0}} & \text { vector of the equilibrium point } \\ \mathbf{\mathbf { x }} & \text { vector of small perturbations } \\ \mu & \text { friction coefficient } \\ \mu_{0} & \text { friction coefficient at the Hopf bifurcation point } \\ \hat{\mu} & \text { small perturbation of the firction coefficient near the equilibrium point } \\ \mathbf{M} & \text { mass matrix } \\ \mathbf{C} & \text { damping matrix } \\ \mathbf{K} & \text { stiffness matrix } \\ \mathbf{y} & \text { state variables vector } \\ \mathbf{v}_{\mathbf{c}} & \text { center variables vector } \\ \mathbf{v}_{\mathbf{s}} & \text { stable variables vector } \\ \mathbf{h} & \text { polynomial approximation of the stable variable in center variables } \\ \mathbf{h}^{(\mathbf{k})} & \text { polynomial approximation of the } k^{t h} \text {-order } \\ \mathbf{a}_{\mathbf{i j l}} & \text { coefficients vector of the center manifold approach } \\ a_{k, i j l} & k^{\text {th }} \text {-coefficient of the center manifold approach } \\ {[m / n]_{f}} & \text { fractional approximant of the function } f \\ d_{\alpha \beta} & \text { denominator coefficients of the fractional approximants } \\ n_{\alpha \beta} & \text { numerator coefficients of the fractional approximants } \\ V_{i, j}^{k} & \text { Fourier coefficients for the } k^{t h} \text {-variable } \\ & \end{array}$

\section{References}

[1] Andronov A.A., Chaikin S.E. Theory of Oscillations. Princeton University Press, Princeton N.J. (1949).

[2] ARnold V.I. Mathematical Methods of Classical Mechanics. Springer-Verlag, Berlin (1978).

[3] Baker G.A., Graves-Morris P. Padé Approximants. Cambridge university Press (1996).

[4] Barnejee A.K. Influence of Kinetic Friction on the Critical Velocity of Stick-Slip Motion. Wear, 12, 107-116 (1968).

[5] Bendat J.S., Piersol A.G. Spectral Analysis of Non-Linear Systems Involving Square-Low Operations. Journal of Sound and Vibration, 81, 199-213 (1982).

[6] BI Q., YU P. Computation of Normal Forms of Differential Equations Associeted with Non-Semisimple Zero Eigenvalues. International Journal of Bifurcations and Chaos, 12, 279-319 (1998).

[7] BIRKhoff G.D. Dynamical Systems. A.M.S. Publications, Providence (1927).

[8] Birkhoff G.D. Sur l'Existence de Régions d'Instabilité en Dynamique, vol. 2. Ann. Inst. H. Poincaré (1932).

[9] BLACK R.J. Realistic Evaluation of Airplane Brake Vibration by Laboratory Test and Analysis. ASME Design Engineering Technical Conferences, 3, 1197-1207 (1995). 
[10] BLACK R.J. Self Excited Multi-Mode Vibrations of Aircraft Brakes with Nonlinear Negative Damping. ASME Design Engineering Technical Conferences, 3, 1241-1245 (1995).

[11] Black R.J. Self Excited Multi-Mode Vibrations of Aircraft Brakes with Nonlinear Negative Damping. ASME Design Engineering Technical Conferences, 3, 1241-1245 (1995).

[12] Blair K.B., Krousgrill C.M., Farris T.N. Harmonic Balance and Continuation Techniques in the Dynamic Analysis of Duffing's Equation. Journal of Sound and Vibration, 202(5), 717-731 (1997).

[13] Blaquire A. Nonlinear System Analysis. Academic Press, New-York, London (1966).

[14] Bogoliubov N., Mitropolski A. Asymptotic Methods in the Theory of Nonlinear Oscillations. Gordon and Breach (1961).

[15] Boivin N., Shaw S.W., Pierre C. Non-Linear Modal Analysis of Structural Systems Featuring Internal Resonances. Journal of Sound and Vibration, 182(2), 336-341 (1995).

[16] Bowden F.P., TABOR D. The Friction and Lubrication of Solids. Calrendon Press, Oxford (1950).

[17] Bowden F.P., TABOR D. The Friction and Lubrication of Solids - Part II. Calrendon Press, Oxford (1964).

[18] Brezinski C. Padé Type Approximation and General Orthogonal Polynomials, vol. 50. Birkhauser-Verlag (1980).

[19] Brezinski C. Extrapolation Algorithms and Padé Approximations: a Historical Survey. Applied numerical mathematics, 20, 299-318 (1983).

[20] Brezinski C. History of Continued Fractions and Padé Approximant. Springer, Berlin (1990).

[21] Brezinski C. An Introduction to Padé Approximations. in Curves and Surfaces in Geometric design, 1, 59-65 (1994).

[22] BRujno A.D. Transactions of the Moscow Mathematical Society. Analytical forms of differential equations, 25, 132-198 (1971).

[23] BRujno A.D. Transactions of the Moscow Mathematical Society. Analytical forms of differential equations, 2 (1972).

[24] Burton T.D., Rahman Z. On the Multi-scale Analysis of Srongly Nonlinear Forced Oscillators. International Journal of Non-Linear Mechanics, 21, 135-146 (1986).

[25] Cameron T.M., Griffin J.H. An Alternating Frequency Time Domain Method for Calculating the Steady State Response of Nonlinear Dynamic. Journal of Applied Mechanics, 56, 149-154 (1989).

[26] Cardona A., Coune T., Lerusse A., Géradin M. A Multi-Harmonic Method for Nonlinear Vibration Analysis. International Jounral of Numerical methods in Engineering, 37, 1593-1608 (1991).

[27] Cardona A., Lerusse A., Géradin M. Fast Fourier Nonlinear Vibration Analysis. Computational Mechanics, 22, 128-142 (1998).

[28] CARr J. Application of Center Manifold. Spriner-Verlag, New-York (1981).

[29] Caughey T.K. Equivalent Linearization Techniques. The Journal of the Acousitcal Society of America, 35, 1706-1711 (1963).

[30] CesAri L. Functional Analysis and Galerkin's Method. Michigan MAth., 11, 385-414 (1964).

[31] Chambrette P. Stabilité des Systèmes Dynamiques avec Frottement sec : Application au Crissement des Freins à Disque. Ecole Centrale de Lyon : Student Press (1991). Thèse de Doctorat. 
[32] Chang C.F. The Dynamic Finite Element Modeling of Aircraft Landing System. ASME Design Engineering Technical Conferences, 3, 1217-1226 (1995).

[33] Cheung Y.K., Chen S.H., Lau S.L. Application of the Incremental Harmonic Balance Method to Cubic Non-Linearity Systems. Journal of Sound and Vibration, 140(2), 273-286 (1990).

[34] Chow S.N., Li C.Z., WAng D. Normal Forms and Bifurcation of Planar Vector Fields. Cambridge University Press, Cambridge (1994).

[35] Cochelin B., Damil N., Potier-Ferry M. Asymptotic Numerical Method and Padé Approximants for Non-Linear Elastic Structures. International Journal for Numerical Methods in Engineering, 37, 1187-1213 (1994).

[36] Coyette J.P., Lecomte C., Migeot J.L., Blanche J., Rochette M., Mirkovic G. Calculation of Vibro-Acoustic Frequency Response Functions Using a Single Frequency Boundary Element Solution and a Padé Expansion. Journal of the European Acoustics Association, 85(3), 7-12 (1999).

[37] Craig R., Bampton M.C.C. Coupling of Substructures for Dynamic Analysis. AIAA Journal, 7, 13131321 (1968).

[38] Crolla D.A., Lang A.M. Brake Noise and Vibration - State of Art. Tribologie-Vehicle Tribology, 18, 165-174 (1991).

[39] Cunningham W.J. Introduction to Nonlinear Analysis. McGraw-Hill Book Co., New-York (1958).

[40] Damil N., Potier-Ferry M., Najah A., Chari R., Lahmam H. An Iterative Method Based Upon Padé Approximants. Communications in Numerical Methods in Engineering, 15, 701-708 (1999).

[41] De Montessus de Ballore R. Sur les Fractions Continues Agébriques. Bulletin Soc. Math., France, 30, 28-36 (1902).

[42] Demailly D. Etude du Comportement Non-linéaire dans le Domaine Fréquentiel. Application à la Dynamique Rotor. Ecole Centrale de Lyon : Student Press (2003). Thèse de Doctorat.

[43] Demailly D., Thouverez F., JÉzÉQuel L. Unbalance Response of Rotor/Stator Systems with Nonlinear Bearings by the Time Finite Element Method. IFToMM, 6th International Conference on Rotor Dynamics, September 30-October 3, Sydney, Australia, 1, 1-8 (2002).

[44] Dimitrijevic G. Détermination des Bifurcations de Hopf et des Cycles Limites d'une Structure non Linéaire à un Ecoulement Transsonique d'un Fluide Parfait. Université Pierre et Marie Curie : Student Press (2000). Thèse de Doctorat.

[45] Dowell E.H. Free Vibrations of an Arbitrary Structure in Terms of Component Modes. Journal of Applied Mechanics, 39, 727-732 (1972).

[46] Dowell R.M. Component Mode Analysis of Nonlinear and Nonconservative Systems. Journal of Applied Mechanics, 47, 172-176 (1980).

[47] Draux A. Polynomes Orthogonaux Formels. Applications. Lecture Notes in Mathematics,Springer, Berlin (1983).

[48] D’Souza A.F., Dweib A.H. Self-Excited Vibrations Induced by Dry Friction. Part II: Stability and LimitCycle Analysis. Journal of Sound and Vibration, 1, 177-190 (1990).

[49] EARLES S.W.E., BADI M.N. Oscillatory Instabilities Generated in a Double-Pin and Disc Undamped System: a Mechanism of Disc-Brake Squeal. Proc. I. Mech. E. Conf. on Vibration and Noise in Motor Vehicles, 1, 43-50 (1984). 
[50] Earles S.W.E., Chambers P.W. Disque Brake Squeal Noise Generation: Predicting its Dependency on System Parameters Including Damping. Int. J. of Vehicle design, 8, 538-552 (1987).

[51] EARLES S.W.E., LEE C.K. Instabilities Arising from the Frictional Interaction of a Pin-Disc System Resulting in Noise Generation. Trans. ASME J. Engng Ind., 1, 81-86 (1976).

[52] Earles S.W.E., Soar G.B. Squeal Noise in Disc Brakes. Proc. I. Mech. E. Conf. on Vibration and Noise in Motor Vehicles, paper C100/71 (1971).

[53] Earles S.W.E., Soar G.B. A Vibrational Analysis of a Pin-Disc System with Particular Reference to Squeal Noise in Disc Brakes. Stress Analysis Group Annual Conference, 1, 237-251 (1974).

[54] Elphick C., Tirapegui E., Brachet M.E., Coullet P., Iooss G. A Simple Global Characterisation for Normal Forms of Singular Vector Fields. Université de Nice (1986).

[55] Emaci E., Vakakis A.F., Andrianov I.V., Mikhlin Y. Stufy of Two-Dimensional Axisymmetric Breathers Using Padé Approximants. Nonlinear Dynamics, 13, 327-338 (1997).

[56] Evan-Iwanows Ki R.M. Resonance Oscillations in Mechanical Systems. Elsevier Scientific Publishing Co., New-York (1976).

[57] EwIns D.J. Modal Testing: Theory and Practice. John Wiley \& Sons, New-York (1984).

[58] Feld D.J., Fehr D.J. Complex Eigenvalue Analysis Applied to an Aircraft Brake Vibration Problem. ASME Design Engineering Technical Conferences, 3, 1135-1142 (1995).

[59] Gao C., Kuhlmann-Wilsdorf D., Makel D.D. The Dynamic Analysis of Stick-Slip Motion. Wear, 173, 1-12 (1994).

[60] GeKKer F.R., Khairaliev S.I. On Self-Excited Friction Vibrations in Brakes. ASME Design Engineering Technical Conferences, 3, 1229-1231 (1995).

[61] Gibert C. Analyse Modale Expérimentale Non-Linéaire. Ecole Centrale de Lyon : Student Press (2001). Thèse de Doctorat.

[62] Girardot D. Stabilité et Bifurcations Dynammiques des Systèmes Discrets Autonomes Régulier et avec Chocs. Ecole Polytechnique : Student Press (1997). Thèse de Doctorat.

[63] Gordon J.T.and Merchant H.C. An Asymptotic Method for Predicting Amplitudes of Nonlinear Wheel Shimmy. Journal of Aircraft, 15, 155-159 (1978).

[64] Gutknecht M.H. The Unsymmetric Lanczos Algorithms and their Relations to Padé Approximation, Continued Fractions, and the qd Algortihm. proceedings of the Copper Mountain Conference on Iterative Methods (1990).

[65] GUYAn R.J. Reduction of Stiffness and Mass Matrices. AIAA Journal, 3(2), 380 (1965).

[66] Hale A.L., Meirovitch L. A General Procedure for Improving Substructures Representation in Dynamic Synthesis. AIAA Journal, 84, 269-287 (1982).

[67] Hale A.L., Meirovitch L. A Procedure for Improving Discrete Substructure Representation in Dynamic Synthesis. AIAA Journal, 20, 1123-1136 (1982).

[68] Hamzeh O.N., Tworzydlo W.W., Chang H.J., Fryska S.T. Analysis of Friction-Inducted Instabilites in a Simplified Aircraft Brake. Society of Automotive Engineers (1999).

[69] Hayashi C. Nonlinear Oscillations in Physical Systems. McGraw-Hill Book Co., New-York (1964).

[70] Holmes P., Guckenheimer J. Nonlinear Oscillations, Dynamical Systems, and Bifurcations of Vector Fields. Springer-Verlag (1986). 
[71] Hou S.N. Review of Modal Synthesis Techniques and a New Approach. Schock and vibration Bulletin, 40, 25-30 (1969).

[72] Hsu L. Analysis of Critical and Post-Critical Behaviour of Non-Linear Dynamical Systems by the Normal Form Methode. Part I : Normalisation Formulae. Journal of Sound and Vibration, 89, 169-181 (1983).

[73] Hsu L. Analysis of Critical and Post-Critical Behaviour of Non-Linear Dynamical Systems by the Normal Form Methode. Part II : Divergence and Flutter. Journal of Sound and Vibration, 89, p183-194 (1983).

[74] Hugues Jones R. General Rational Approximants in N-variables. J. Approx. Theory, 7(6), 201-233 (1976).

[75] Hugues Jones R., Makinson G.J. The Generation of Chisholm Rational Approximants to Power Series in Two Variables. J. Inst. Math. Appl.Approx. Theory, 13, 299-310 (1974).

[76] Hulten J. Brake Squeal - A Self-Exciting Mechanism with Constant Friction. Society of Automotive Engineers, paper 932965 (1993).

[77] HurTy W.C. Dynamic Analysis of Structural Systems Using Component Modes. AIAA Journal, 3, 678-685 (1965).

[78] Hwang J.L., Shiau T.N. An Application of the Generalized Polynomial Expansion Method to Nonlinear Rotor Bearing Systems. Journal of Vibration and Acoustics, 113, 299-308 (1991).

[79] Ibrahim R.A. Friction-Induced Vibration, Chatter, Squeal, and Chaos. Part 1 : Mechanics of Contact and Friction. ASME Design Engineering Technical Conferences, 7, 209-226 (1994).

[80] Ibrahim R.A. Friction-Induced Vibration, Chatter, Squeal, and Chaos. Part 2 : Dynamics and Modeling. ASME Design Engineering Technical Conferences, 7, 226-269 (1994).

[81] Imbert J.F. Analyse des Structures par Elements Finis. Cepadues (1991). In french.

[82] Ioss G. Local Techniques in Bifurcation Theory and Nonlinear Dynamics. Chaotic Motions in Nonlinear Dynamical Systems, Springer, 1, 137-193 (1988).

[83] Ioss G., Adelmeyer M. Topics in Bifurcation Theory and Applications. Advanced series in nonlinear dynamics vol3 - World Scientific Singapore-New-Jersey-London-Hong Kong (1992).

[84] Ioss G., Vanderbauwhede A. Center Manifold Theory in Infinite Dimensions. Dynamics reported 1 new series-Springer Verlag (1992).

[85] Iwan W.D. Application of an Equivalent Nonlinear System Approach to Dissipative Dynamical Systems. Journal of Applied Mechanics, 1, 412-416 (1969).

[86] Iwan W.D. On Defining Equivalent Systems for Certain Ordinary Non-Linear Differential Equations. International Journal of Non-linear mechanics, 4, 325-334 (1969).

[87] IWAN W.D. A Generalization of the Concept of Equivalent Linearization. International Journal of Non-linear mechanics, 8, 279-287 (1973).

[88] Iwan W.D., Krousgrill C.M. Equivalent Linearization for Continuous Dynamical Systems. Journal of Applied Mechanics, 50, 415-420 (1983).

[89] IWAn W.D., YAng I.M. Application of Statitical Linearization Techniques to Nonlinear Multidegree-ofFreedom Systems. Journal of Applied Mechanics, 50, 545-550 (1972).

[90] JARVis R.P., Mills B. Vibrations Induced by Dry Friction. Proc. Instn. Mech. Engrs, 132, 847-866 (1993).

[91] Jean A.N., Nelson H.D. Periodic Response Investigation of Large Order Nonlinear Rotordynamic Systems Using Collocation. Journal of Sound and Vibration, 143(3), 299-308 (1990). 
[92] Jezequel L., Lamarque C.H. Analysis of Non-Linear Dynamical Systems by the Normal Form Theory. Journal of Sound and Vibration, 149, 429-459 (1991).

[93] Joseph D.D., Iooss G. Elementary Bifurcation and Stability Theory. Berlin: Springer-verlag (1980).

[94] JÉZÉQuel L. Synthèse Modale - Théorie et Extensions. Université Claude-Bernard-Lyon 1: Student Press (1985). Thèse de Doctorat ès-Sciences.

[95] Kanarachos A.E., Spentzas C.N. A Galerkin Method for the Steady State Analysis of Harmonically Excited Non-linear Systems. Mechanisms and machine Theory, 27, 661-671 (1997).

[96] Kantarovich L.V., Krylov V.L. Approximate Methods of Higher Analysis. P.Noordhoff Ltd., Groninger (1958).

[97] KIM Y-B. Quasi-periodic Response and Stability Analysis for Non-linear Systems: a General Approach. Journal of Sound and Vibration, 192(4), 821-833 (1996).

[98] KIM Y-B. Multiple Harmonic Balance Method for Aperiodic Vibration of a Piecewise-Linear System. Journal of Vibration and Acoustics, 120, 181-187 (1998).

[99] Knoblock H.W. Construction of Center Manifolds. ZAMM Z angew. Math. Mech., 70, 215-233 (1990).

[100] Kobayashi M. Sound and Vibration in Brakes. Japanese Journal of Tribology, 35, 561-567 (1990).

[101] Krylov N.M., Bogoliubov N.N. Introduction to Nonlinear Mechanics. Princeton (1947).

[102] Kusano M., Ishidou H., Matsumurz S., Washizu S. Experimental Study on the Reduction of Drum Brake Noise. Society of Automotive Engineers, paper 851465 (1985).

[103] Lamarque C.H. Modélisation et Identification des Systèmes Mécaniques Non Linéaires. Ecole Centrale de Lyon: Student Press (1992). Thèse de Doctorat.

[104] Lamnabhi-Lagarrigue F. Analyse des Systèmes Non Linéaires. Hermes (1994).

[105] Lang A.M., Newcomb T.P. An Experimental Investigation into Drum Brake Squeal. I. Mech.E/EAEC Conf., paper C382/051 (1989).

[106] Larsson H., Farhang K. Investigation of Stick-Slip Phenomenon Using a Two-Disk Friction System Vibration Model. ASME Design Engineering Technical Conferences, 1, 1-10 (1997).

[107] Lau S.L., Cheung Y.K. Amplitude Incremental Variational Principle for Nonlinear Vibration of Elastic Systems. Journal of Applied Mechanics, 28, 959-964 (1981).

[108] Lau S.L., Cheung Y.K., Wu S.Y. A Variable Parameter Incrementation Method for Dynamic Instability of Linear and Nonlinear Amplitude Incremental Variational Principle for Nonlinear Vibration of Linear and Nonlinearb Elastic Systems. Journal of Applied Mechanics, 49, 849-853 (1982).

[109] Lau S.L., Cheung Y.K., Wu S.Y. Incremental Harmonic Balance with Multiple Time Scales for Aperiodic Vibrations of Nonlinear Systems. Journal of Applied Mechanics, 50, 871-876 (1983).

[110] LaU S.L., Zhang W.S. Non-Linear Vibration of Piecewise-Linear Systems by Incremental Harmonic Balance Method. Journal of Applied Mechanics, 59, 153-160 (1992).

[111] Leung A.Y.T., Chui S.K. Non-Linear Vibration of Coupled Duffing Oscillators by an Improved Incremental Harmonoc Balance Method. Journal of Sound and Vibration, 181(4), 619-633 (1995).

[112] Liapunov A.M. Problèmes Général de la Stabilité du Mouvement. Annals of Mathematical Studies, princeton University press, Princeton (1949). 
[113] Ling F.H., Wu X.X. Fast Galerkin Method and its Application to Determine Periodic Solutions of Nonlinear Oscillators. International Journal of Nonlinear Mechanics, 22, 89-98 (1987).

[114] Liu Y.S., Ozbek M.A., Gordon J.T. A Nonlinear Model for Aircraft Brake Squeal Analysis. Part I : Model Description and Solution Methodology. ASME Design Engineering Technical Conferences, 3, 406-416 (1996).

[115] Malkin N.G. Some Problems of the Theory of Nonlinear Oscillations. OTS Atomic Energy Commission, Washington DC (1962).

[116] Marsden J.E., McCracken M. The Hopf Bifurcation and its Applications. New-York: Springer-verlag (1976).

[117] Meirovitch L. Elements of Vibration Analysis. Mac Graw Hill International Editions (1986).

[118] Meirovitch L. Dynamics and Control of Structures. John Wiley \& Sons (1990).

[119] Meirovitch L., Hale A.L. On the Substructure Synthesis Method. AIAA Journal, 19, 940-947 (1981).

[120] Mickens R.E. An Introduction to Nonlinear Oscillations. Cambridge University Press, Springer-Verlag (1971).

[121] Millner N. An Analysis of Disc Brake Squeal. SAE, paper 780332 (1978).

[122] Millner N., Parsons B. Effect of Contact Geometry and Elastic Deformations on the Torque Characteristics of a Drum Brake. Proc. Instn. Mech. Engrs, 187, 317-331 (1973).

[123] Mills H.R. Brake Squeal. Institution of Automobile Engineers, Research Report 9000b and Research Report 9162B (1938).

[124] Moirot F. Etude de la Stabilité d'un Equilibre en Présence de Frottement de Coulomb. Application à l'Etude des Freins à Disque. Ecole Polytechnique : Student Press (1998). Thèse de Doctorat.

[125] Moser J. Stable and Random Motion in Dynamical Systems. Hermann Weyl Lectures, Princeton, New Jersey (1973).

[126] Murray D. Normal Form Investigations of Dissipative Systems. Mechanics Research Communications, 21(3), 231-240 (1994).

[127] NACK W.C. Brake Squeal Analysis by Finite Elements and Comparisons to Dyno Results. ASME Design Engineering Technical Conferences, pp. 1-8 (1999).

[128] Nakai M., Yokoi M. Band Brake Squeal. Journal of Vibration and Acoustics, 118, 190-197 (1996).

[129] Narayanan S., Sekar P. A Frequency Domain Based Numeric-Analytical Method for Non-Linear Dynamical Systems. Journal of Sound and Vibration, 211(3), 409-424 (1998).

[130] Nataraj C., Nelson H.D. A Collocation Method for the Investigation of Periodic Solutions in Nonlinear Systems. 12th Design Technical Conference on Mechanical Vibration and Noise- Diagnostics, vehicle Dynamics and Special Topics, Montréal, Canada, 1, 325-330 (1989).

[131] Nataraj C., Nelson H.D. Periodic Solutions in Rotor Dynamic Systems With Nonlinear Supports: A General Approach. Journal of Vibration and Acoustics, 111, 187-193 (1989).

[132] NAYFeH A.H. Introduction to Perturbation Techniques. John Wiley \& Sons, New-York (1981).

[133] NAYFeH A.H. Methods of Normal Forms. John Wiley \& Sons, New-York (1993).

[134] Nayfeh A.H., Balachandran B. Applied Nonlinear Dynamics : Analytical, Computational and Experimental Methods. John Wiley \& Sons (1995). 
[135] NAYfeh A.H., Mook D.T. Nonlinear Oscillations. John Wiley \& Sons (1995).

[136] North M.R. A Mechanism of Disk Brake Squeal. 14th FISITA Congress, 1/9 (1972).

[137] Oden J.T., Martins J.A.C. Models and Computational Methods for Dynamic Friction Phenomena. Computer Methods in Applied mechanics and Engineering, 52, 527-634 (1985).

[138] Ostachowicz W. The Harmonic Balance Method for Determining the Vibration Parameters in Damped Dynamic Systems. Journal of Sound and Vibration, 131(3), 465-473 (1989).

[139] OzBex M.A. A Pertubation Analysis of Nonlinear Squeal Vibrations in Aircraft Braking Systems. In ASME Design Engineering Technical Conferences, vol. 3, pp. 1-10 (1997).

[140] Padé H. Sur la Représentation Approchée d'une Fonction par des Fractions Rationnelles. Ann. Sci. Ecole Normale Supérieure, Oeuvres 4, Berlin, 9, 1-93 (1892).

[141] Pellicano F., Mastroddi F. Applicability Conditions of a Non-linear Superpostion Technique. Journal of Sound and Vibration, 200(1), 3-14 (1997).

[142] Pierre C., Dowell E.H. A Study of Dynamic Instability of Plates by an Extended Incremental Harmonic Balance Method. Journal of Applied Mechanics, 52, 993-997 (1985).

[143] Pierre C., Ferri A.A., Dowell E.H. Multi-Harmonic Analysis of Dry friction Damped Systems Using an Incremental Harmonic Balance Method. Journal of Applied Mechanics, 56, 958-964 (1985).

[144] Poincaré H. Mémoire sur les Courbes Définies par les Equations Différentielles i-VI, Oeuvres I. GauthierVillar, Paris (1880).

[145] Poincaré H. Les Méthodes Nouvelles de la Mécanique Céleste. Librairie A. Blanchard, Paris (1899).

[146] Poincaré H. Oeuvres 1. Thèse, Paris, année 1928 (1979).

[147] Qinsheng B., Yu P. Double Hopf Bifurcation and Chaos of a Nonlinear Vibration System. Nonlinear Dynamics, 19, 313-332 (1999).

[148] RabinowicZ. Friction and Wear of Materials. Wiley \& Sons (1965).

[149] Raghothama A., Narayanan S. Non-Linear Dynamics of a Two-Dimensional Airfoil by Incremental Harmonic Balance Method. Journal of Sound and Vibration, 226(3), $493-517$ (1999).

[150] Remington P.J. Wheel Rail/Noise. Part I: Characterization of the Wheel/Rail Dynamic System. Journal of Sound and Vibration, 46(3), 359-379 (1976).

[151] Ren Y., Beards C.F. A New Receptance-Based Perturbative Multi-Harmonic Balance Method for the Calculation of the Steady State Response of Non-Linear Systems. Journal of Sound and Vibration, 172, 593604 (1994).

[152] Rocard Y. Dynamique Générale des Vibrations. Masson (1971). In french.

[153] Rosenberg R.M. The Normal Modes of Nonlinear n-Degree-of-Freedom Systems. ASME Journal of Applied Mechanics, 18, 7-14 (1962).

[154] Rosenberg R.M. On Non-Linear Vibrationsof Systems with Many Degree of Freedom. Advances in Applied Mechanics, 9, 155-242 (1966).

[155] Roy R.V., Spanos P.D. Padé Type Approach to Nonlinear Random Vibration Analysis. Engineering probabilistic Mechanics, 1, 119-128 (1991).

[156] Rudowski J. Limit Cycles in Self-Excited Multi-Degree-of-Freedom Systems. Journal of Sound and Vibration, 81(1), 33-49 (1982). 
[157] SAito S. Calculation of Nonlinear Unbalance Response of Horizontal Jeffcott Rotors Supported by Ball Bearings with Radial Clearances. Journal of Vibration, Acoustics, Stress and Reliability in Design, 107(4), 416-420 (1985).

[158] Samoilenko A.M., Ronto N.I. Numerical Analytic Methods of Investigating Periodic Solutions. Mir Publishers, Moscou, 1 (1979).

[159] Sanders J.A., Verhulst F. Averaging Methods in Nonlinear Dynamical Systems. Applied Mathematical Sciences, Springer-Verlag (1985).

[160] Setio S. Comportement des Structures Mécaniques Non-Linéaires Soumises à des Excitations Stationnaires. Ecole Centrale de Lyon : Student Press (1991). Thèse de Doctorat.

[161] Setio S., Setio H.D., JÉzÉquel L. A Method of Non-Linear Modal Identification From Frequency Response Tests. 8th International Modal Analysis Congress, Orlando, Florida, 1, 334-340 (1990).

[162] Setio S., Setio H.D., JÉzÉquel L. A Method of Non-Linear Modal Identification From Frequency Response Tests. Journal of Sound and Vibration, 158(3), 497-515 (1992).

[163] Shaw S.W., PiERre C. Non-Linear Normal Modes and Invariant Manifolds. Journal of Sound and Vibration, 150(1), 170-173 (1991).

[164] Shaw S.W., Pierre C. Normal Modes for Non-Linear Vibratory Systems. Journal of Sound and Vibration, 164(1), 85-124 (1993).

[165] Shaw S.W., Pierre C. Normal Modes of Vibration for Non-Linear Continuous Systems. Journal of Sound and Vibration, 150(1), 319-347 (1994).

[166] Sinclair D., Manville N.J. Frictional Vibrations. Journal of Applied Mechanics, pp. 207-213 (1955).

[167] Sinou J-J. Synthèse Non-linéaire des Systèmes Vibrants: Application aux Systèmes de Freinage. Ecole Centrale de Lyon: Student Press (2003). Thèse de Doctorat.

[168] Sinou J-J., Thouverez F., Dereure O., Mazet G.B. Non-linear Dynamics of a Complex Aircraft Brake System - Experimental and Theoretical Approaches. ASME Design Engineering Technical Conferences and Computers and Information in Engineering Conference, 19th Biennal Conference on Mechanical Vibration and Noise, Chicago, Illinois, September 2-6, 1, 1-10 (2003).

[169] Sinou J-J., Thouverez F., JÉzÉQuel L. Application of Multivariable Approximants to Stability of Dynamical Systems with Polynomial Non-Linearities. 5th EUROMECH Conferences on Nonlinear Oscillations, Moscow, Russia, August 9-23, 1, 44 (2002).

[170] Sinou J-J., Thouverez F., JÉzÉquel L. Center Manifold Approach and Multivariable Approximants Applied to Non-Linear Stability Analysis. International Journal of Non-Linear Mechanics, 38(9), 1421-1442 (2002).

[171] Sinou J-J., Thouverez F., JÉzÉQuel L. Stability Analysis of Nonlinear Dynamical Systems Based on the Normal Form Theory. 5th EUROMECH Conferences on Nonlinear Oscillations, Moscow, Russia, August 9-23, 1, 9 (2002).

[172] Sinou J-J., Thouverez F., JÉzÉquel L. Analysis of Friction and Instability by the Center Manifold Theory for an Non-Linear Sprag-Slip Model. Journal of Sound and Vibration, 265(3), 527-559 (2003).

[173] Sinou J-J., Thouverez F., JÉzÉQuel L. Dynamique Non-linéaire d'un Ensemble Rotor/Stator Comportant des Mécanismes Non-linéaires avec Jeu. Proceedings du 6ème Colloque National en Calcul des Structures,May 20-23, Giens, 1, 1-10 (2003). 
[174] Sinou J-J., Thouverez F., JÉzÉquel L. Extension of the Center Manifold Approach, Using the Rational Fractional Approximants, Applied to Non-linear Stability Analysis. Journal of Nonlinear Dynamics, 33, 267282 (2003).

[175] Sinou J-J., Thouverez F., JÉzÉQuel L. Non-Linear Stability Analysis Based on the Center Manifold Approach, the Padé Approximants and the Alternate/Frequency Time Domain Method. 8th International Conference on Recent Advances in Structural Dynamics, Southampton, United Kingdom, July 14-16, 1, 1-12 (2003).

[176] Sinou J-J., Thouverez F., JÉzÉQuel L. Application of a Nonlinear Modal Instability Approach to Brake Systems. Journal of Vibration and Acoustics, 126(1), 101-107 (2004).

[177] Spanos P-T.D., IwAn W.D. On The Existence and Uniqueness of Solutions Generated by Equivalent Linearization. International Journal of Non-linear mechanics, 13, 71-78 (1978).

[178] Spanos P-T.D., IwAn W.D. Harmonic Analysis of Dynamic Systems With Nonsymmetric Nonlinearities. Journal of Dynamic Systems, Measurement, and Control, 101, p31-36 (1979).

[179] SPurR R.T. A Theory of Brake Squeal. Proc. Auto. Div. Instn. Mech. Engrs, 1, 33-40 (1961).

[180] Stewart G.W., Sun J.G. Computer Science and Scientific Computing. Matrix Perturbation Theory, Academic press (1990).

[181] Stieltjes T.G. Sur la Réduction en Fraction Continue d'une Série Procédant suivant les Puissances Descendantes d'une Variables. Annales Faculté des Sciences de Toulouse, France, 3, 1-17 (1889).

[182] Stieltues T.G. Recherches sur les Fractions Continues. Annales Faculté des Sciences de Toulouse, France, 8, 1-122 (1894).

[183] Stoker J.J. Nonlinear Vibrations in Mechanical and Electrical Systems. Int. Publ. Inc., New-york (1950).

[184] Stokes A. On the Approximation of Non-linear Oscillations. Journal of Differential Equations, 12, 535-558 (1972).

[185] Sundararajan P., Noah S.T. Dynamics of Forced Nonlinear Systems UsingShooting/Arc-length Continuation Method - Application to Rotor Systems. Journal of Vibration and Acoustics, 119, 9-20 (1997).

[186] Szemplinska-Stupnicka W. The Modified Single Mode Method in the Investigation of the Resonant Vibration of Non-Linear Systems. Journal of Sound and Vibration, 104(2), 475-489 (1979).

[187] Szemplinska-Stupnicka W. Non-Linear Normal Modes and the Generalized Ritz Method in the Problems in Vibrations of Non-Linear Elastic Continuous Systems. International journal of Non-linear Mechanics, 18, p149-165 (1983).

[188] Szemplinska-Stupnicka W. The Behaviour of Nonlinear Vibrating Systems: Advanced Concepts and Application to Single-Degree-of-Freedom Systems. Kluwer Academic Publishers, Dordrecht, 1, 330 (1990).

[189] Szemplinska-Stupnicka W. The Behaviour of Nonlinear Vibrating Systems: Fundamental Concepts and Methods. Kluwer Academic Publishers, Dordrecht, 1, 253 (1990).

[190] Tolstoi D.M. Significance of the Normal Degree of Freedom and Natural Normal Vibrations in Contact Friction. Wear, 10, 199-213 (1967).

[191] TondL A. On the Internal Resonance of a Nonlinear System with two Degrees of Freedom. Nonlinear Vibration Problems, 5, 207-222 (1963).

[192] Tondl A. Domains of Attraction for Nonlinear Systems. National Research Inst. for machine Design, bechovice, Monographs and Memoranda, 8 (1970). 
[193] Tondl A. Quenching of Self-excited Vibrations: Equilibrium Aspects. Journal of Sound and Vibration, 42, 251-260 (1975).

[194] TondL A. Quenching of Self-excited Vibrations: One and Two-frequency Vibrations. Journal of Sound and Vibration, 42, 261-271 (1975).

[195] Travis M.H. Nonlinear Transient Analysis of Aircraft Landing Gear Brake Whirl and Squeal. ASME Design Engineering Technical Conferences, 3, 1209-1216 (1995).

[196] Urabe M. Galerkin's Procedure for Nonlinear Periodic Systems. Archive Rational Mech. Anal., 20, 120-152 (1965).

[197] Urabe M., Reiter A. Numerical Computation of Nonlinear Forced Oscillations by Galerkin's Procedure. Journal of Math. Anal. Appl., 14, 107-140 (1966).

[198] Vakakis A.F., Manevitch L.I., Mikhlin Y.V. AndPilipchuck V.N., Zevin A.A. Normal Modes and Localization in Nonlinear Systems. John Wiley \& Sons (1996).

[199] Vola D., Raous M., Martins A.C. Friction and Instability of Steady Sliping:Squeal of Rubber/Glass Contact. International Journal for Numerical Methods in Engineering, 1, 1699-1720 (1999).

[200] Wang Z., Hu H. Robust Stability Test for Dynamic Systems with Short Delays by Using Padé Approximation. Nonlinear Dynamics, 18, 275-287 (1999).

[201] WAZWA A.M. Analytical Approximations and Padé Approximants for Volterra's Population Model. Applied Mathematics and Computation, 100, 13-25 (1999).

[202] XIE G., LOU J.Y.K. Alternating Frequency/Coefficient (AFC) Technique in the Trigonometric Collocation Method. International Journal of Non-Linear Mechanics, 31(4), 531-545 (1996).

[203] YU P. Computation of Normal Forms via a Perturbation Technique. Journal of Sound and Vibration, 211, 19-38 (1998).

[204] YU P., YUAN Y. The Simplest Normal Forms Associated with a Triple Zero Eigenvalue of Indices one and two. Nonlinear Analysis, 47, 1105-1116 (2001).

[205] YUAN. A Study of the Effects of Negative Friction-Speed Slope on Brake Squeal. ASME Design Engineering Technical Conferences, 3, 1153-1162 (1995).

[206] Zhang W., Huseyin K. Computation of the Coefficients Associated with the Normal Form of a Rsonant Five-Dimensional System. Mathematical and Computer Modelling, 30, 213-228 (1999). 